Florida International University

FIU Digital Commons

FIU Electronic Theses and Dissertations

University Graduate School

$11-14-2017$

\title{
Numerical, Analytical, and Experimental Studies of Reciprocating Mechanism Driven Heat Loops for High Heat Flux Cooling
}

Olubunmi Tolulope Popoola

Florida International University, opopo002@fiu.edu

DOI: $10.25148 /$ etd.FIDC004065

Follow this and additional works at: https:// digitalcommons.fiu.edu/etd

Part of the Heat Transfer, Combustion Commons, and the Other Mechanical Engineering Commons

\section{Recommended Citation}

Popoola, Olubunmi Tolulope, "Numerical, Analytical, and Experimental Studies of Reciprocating Mechanism Driven Heat Loops for High Heat Flux Cooling" (2017). FIU Electronic Theses and Dissertations. 3505.

https://digitalcommons.fiu.edu/etd/3505 


\title{
FLORIDA INTERNATIONAL UNIVERSITY
}

Miami, Florida

\section{NUMERICAL, ANALYTICAL, AND EXPERIMENTAL STUDIES OF RECIPROCATING MECHANISM DRIVEN HEAT LOOPS FOR HIGH HEAT FLUX COOLING}

\author{
A dissertation submitted in partial fulfilment of \\ the requirements for the degree of \\ DOCTOR OF PHILOSOPHY \\ in \\ MECHANICAL ENGINEERING \\ by \\ Olubunmi Tolulope Popoola
}


To: Dean John L. Volakis

College of Engineering and Computing,

This dissertation, written by Olubunmi Tolulope Popoola, Numerical, Analytical, and Experimental Studies of Reciprocating Mechanism Driven Heat Loops for High Heat Flux Cooling, having been approved in respect to style and intellectual content, is referred to you for judgment.

We have read this dissertation and recommend that it be approved.

$\begin{array}{r}\text { George Dulikravich } \\ \hline \text { Cheng-Xian Lin } \\ \hline \text { Walter Tang } \\ \hline \text { Yiding Cao, Major Professor Nur Tansel }\end{array}$

Date of Defense: November 14, 2017

The dissertation of Olubunmi Tolulope Popoola is approved.

Dean John L. Volakis

College of Engineering and Computing,

Andrés G. Gil

Vice President for Research and Economic Development and Dean of the University Graduate School

Florida International University, 2017 
(C) Copyright 2017 by Olubunmi Tolulope Popoola

All rights reserved. 


\section{DEDICATION}

I dedicate this dissertation to my family for all of their love and support. I would not be as ambitious or successful without your encouragement. 


\author{
ABSTRACT OF THE DISSERTATION \\ NUMERICAL, ANALYTICAL, AND EXPERIMENTAL STUDIES OF \\ RECIPROCATING MECHANISM DRIVEN HEAT LOOPS FOR HIGH \\ HEAT FLUX COOLING \\ by \\ Olubunmi Tolulope Popoola \\ Florida International University, 2017 \\ Miami, Florida \\ Professor Yiding Cao, Major Professor
}

The Reciprocating Mechanism Driven Heat Loop (RMDHL) is a novel heat transfer device that utilizes reciprocating flow, either single-phase or two-phase flow, to enhance the thermal management in high tech inventions. The device attains a high heat transfer rate through a reciprocating flow of the working fluid inside the heat transfer device. Although the concept of the device has been tested and validated experimentally, analytical or numerical studies have not been undertaken to understand its working mechanism and provide guidance for the device design. The objectives of this study are to understand the underlying physical mechanisms of heat transfer in internal reciprocating flow, formulate corresponding heat transfer correlations, conduct an experimental study for the heat transfer coefficient, and numerically model the single-phase and two-phase operations of the RMDHL to predict its performance under different working conditions. The two-phase flow boiling model was developed from the Rensselaer Polytechnic Institute (RPI) model, and a virtual loop written in $\mathrm{C}$ programming language was used to eliminate the need for fluid structure interaction (FSI) modelling. The accuracy of 
several turbulence formulations, including the Standard, RNG, and Realizable k- $\varepsilon$

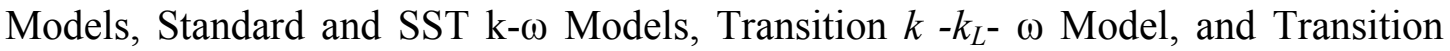
SST Model, have been test in conjunction with a CFD solver to select the most suitable turbulence modelling techniques. The numerical results obtained from the single-phase and two-phase models are compared with relevant experimental data with good agreement. Three-dimensional numerical results indicate that the RMDHL can meaningfully reduce the peak temperature of an electronic device and result in significantly more uniform temperature across the device. In addition to the numerical study, experimental studies in conjunction with analytical studies are undertaken. Experimental data and related heat transfer coefficient as well as practically useful semi-empirical correlations have been produced, all of which provide archival information for the design of heat transfer devices involving a reciprocating flow. In particular, this research will lead to the development of more powerful RMDHLs, achieve a heat flux goal of $600 \mathrm{~W} / \mathrm{cm}^{2}$, and significantly advance the thermal management at various levels. Considering the other advantages of coolant leakage free and the absence of cavitation problems, the RMDHL could also be employed for aerospace and battery cooling applications. 


\section{TABLE OF CONTENT}

CHAPTER

PAGE

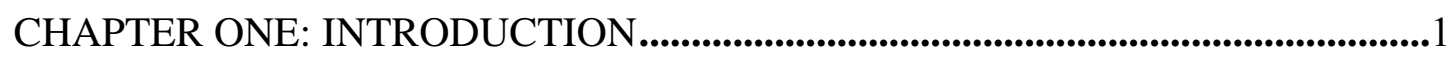

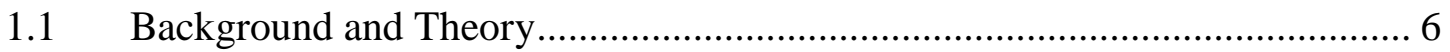

1.2 Reciprocating Heat Transfer in the RMDHL............................................ 12

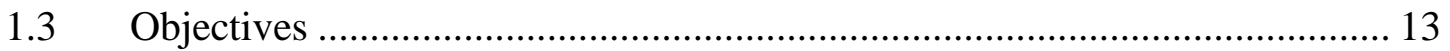

CHAPTER TWO: LITERATURE REVIEW ........................................................... 16

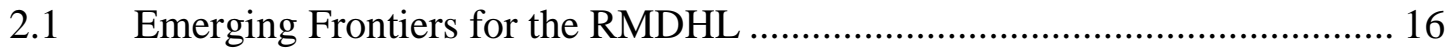

2.1.1 Battery Pack cooling (temperature uniformity) .................................... 16

2.1.2 Liquid cooling system employing a RMDHL with leakage free............... 20

2.1.3 Aerospace (Body forces, cavitation, and high heat flux application) ......... 22

2.1.4 New weapons based on directed energy systems (very high heat flux) ..... 23

2.2 Oscillating Flow Systems and their Analytical Solutions ............................. 23

2.2.1 Dimensionless Numbers associated with Oscillatory Flow ...................... 29

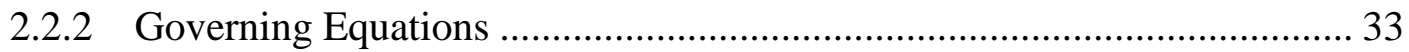

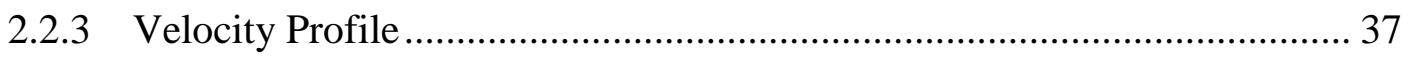

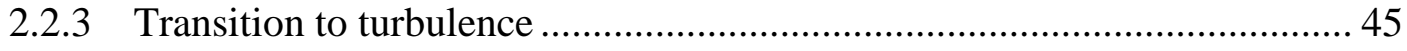

2.2.4 Turbulence Modelling for A Reciprocating Mechanism Driven Heat loop 46 


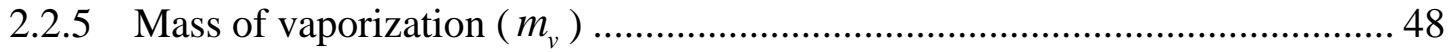

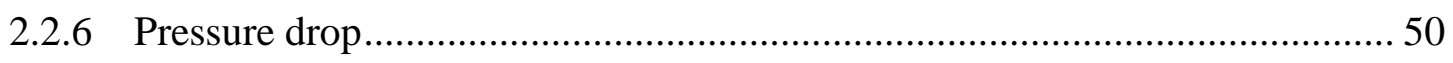

2.2.7 Thermodynamic Principles .................................................................... 51

2.8 Convective Heat Transfer Correlations …………………………………..... 57

CHAPTER THREE: EXPERIMENTAL STUDIES ....................................................... 69

3.1. Design and Construction of a Single-Phase Testing Rigs .............................. 71

3.1.1. Evaporator design for bulk temperature measurements............................. 79

3.1.2. Surface/volume requirement in the condenser section ............................. 82

3.2 Experiment Setup for Single-Phase Heat Transfer Correlation ....................... 83

3.3 Design and Construction of a Two-Phase Experimental Setup ...................... 88

3.4 Single-Phase Heat Transfer Correlations........................................................ 92

3.5. Temperature Measurements of Single-Phase Heat Transfer Experiments ... 104

CHAPTER FOUR: NUMERICAL STUDIES ..................................................... 107

4.1 Governing Equations for Single-Phase Flow............................................... 112

4.2 Two-phase Numerical Models For RMDHL .................................................... 113

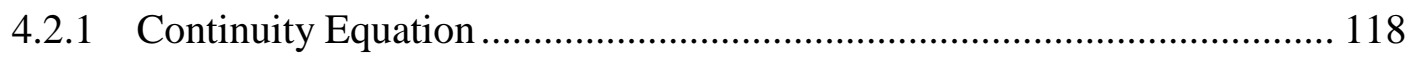


4.2.1.1. Mass Transfer from the Wall to Vapor ............................................ 118

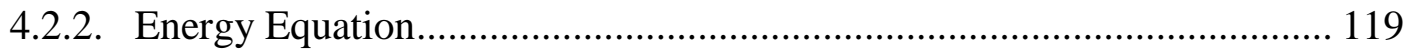

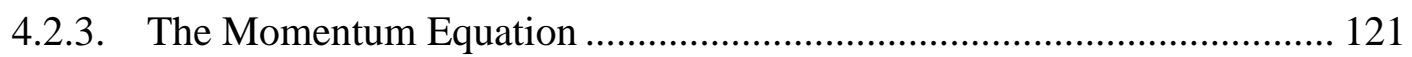

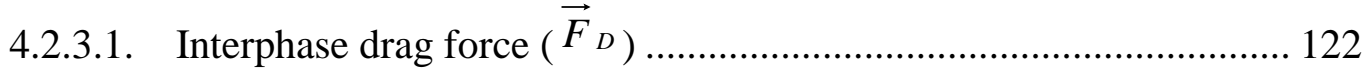

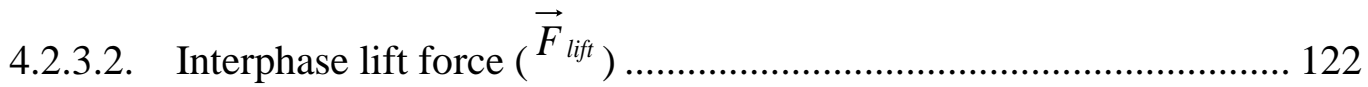

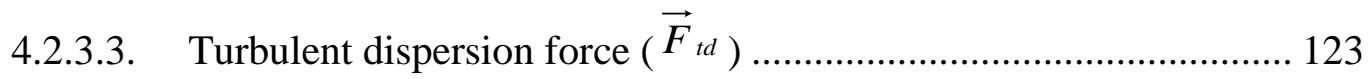

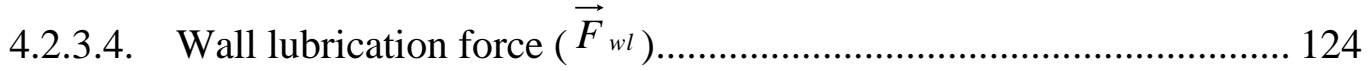

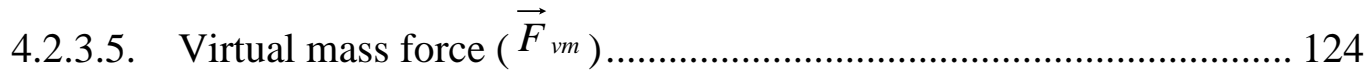

4.2.4. Transport effects at the interface ...................................................... 125

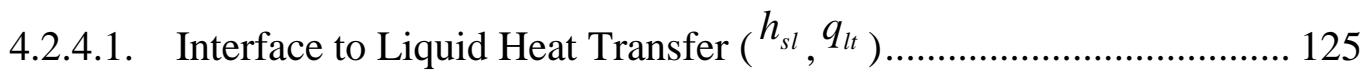

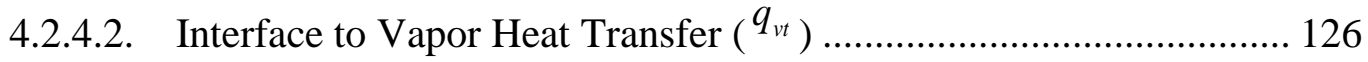

4.2.4.3. Interfacial Mass Transfer .......................................................... 126

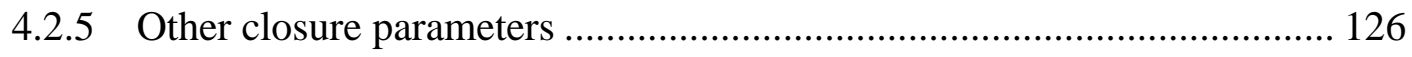

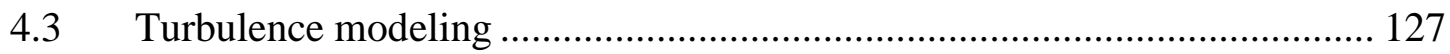

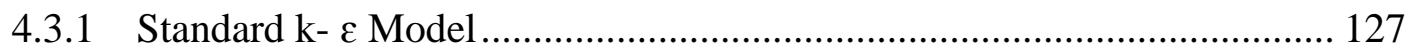

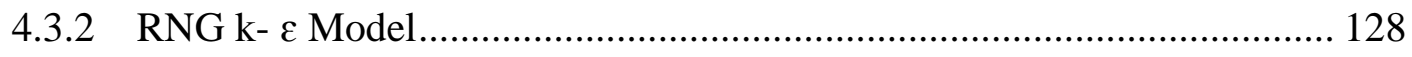

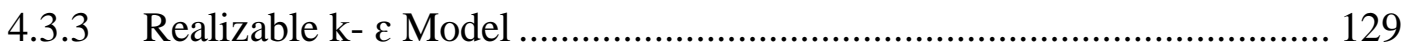

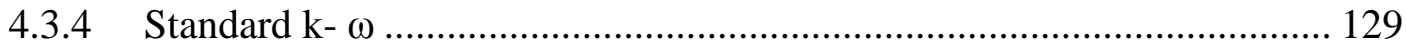

4.3.5 Shear-Stress Transport (SST) k - $\omega$ Model.......................................... 130

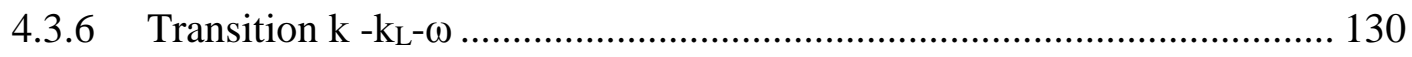


4.3.7 Transition SST Model; ................................................................... 131

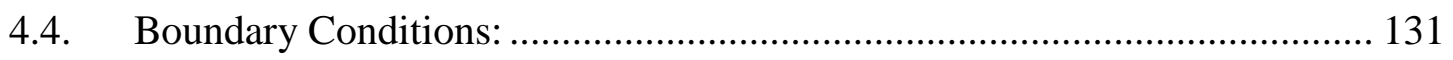

4.5 CAD Meshing, Discretization and Simplification ................................... 132

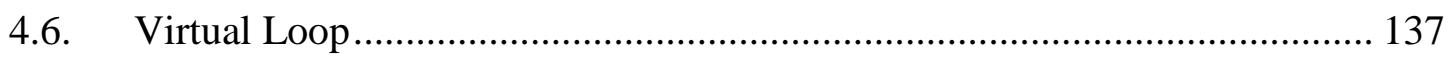

4.7. Empirical method for comparing experimental data and Numerical Res.ults .......................................................... 140

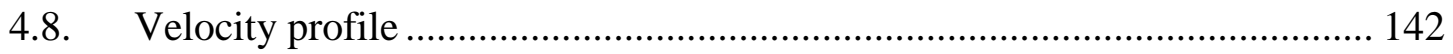

4.8.1 Inlet/outlet conditions specifications .................................................. 142

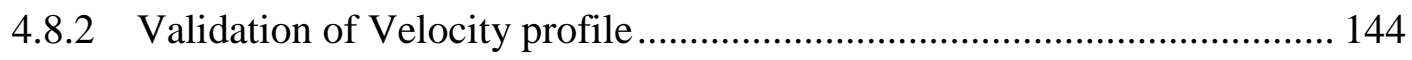

4.9. Comparative Studies into Temperature Uniformity between DPDHL with continuous flow Cooling and Oscillating Flow Cooling using Two-Dimensional Single-Phase Model............................................................. 146

4.10. Investigation into the Effect of various turbulence models on the accuracy of Numerical Model.......................................................................... 159

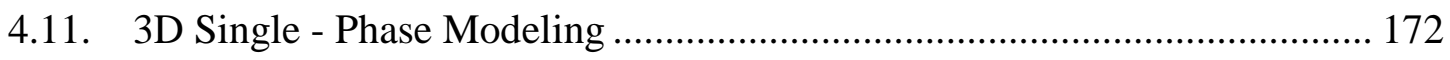

4.12. Results of Two-Phase Heat Transfer Modeling .......................................... 192

4.12.1. Estimating Work done in RMDHL loop using Two-phase model. ...... 207 
4.12.2. Transient Characteristic of Cold plate with Two-phase application

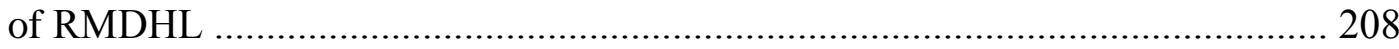

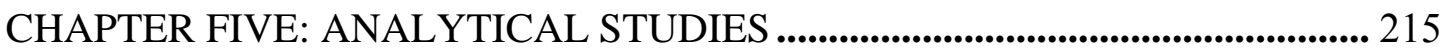

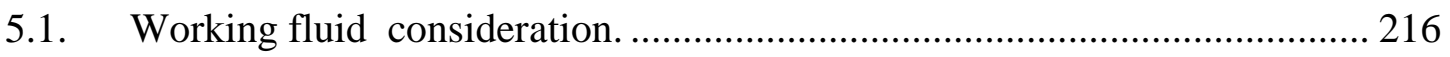

5.2 Pump Selection and Power Analysis for the Oscillating Flow ..................... 220

5.2.1 Critical displacement of the reciprocating driver..................................... 220

5.2.2. Inner surface/volume requirement in the condenser section.................... 223

5.2.3 Interior volume requirement associated with the evaporator.................... 225

5.2.4. Volume associated with the connection tubing ........................................ 225

5.2.5 Liquid displacement volume in the reservoir.......................................... 225

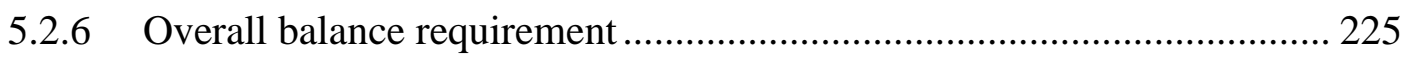

5.2.7 Power consumption of the actuator....................................................... 227

5.2.7.1 Modeling and theoretical considerations ……………………….... 227

5.2.7.2. Mass flow rate induced by the motion of the piston........................ 229

5.2.7.3. Pressure drop in the evaporator section of the cooling loop ............. 231

5.2.7.4. Pressure drop in the connection tubing of the cooling loop.............. 231

5.2.7.5. Pressure drop in the condenser section of the heat loop .................. 232

5.2.7.6. Total pressure drop in the heat loop................................................ 233

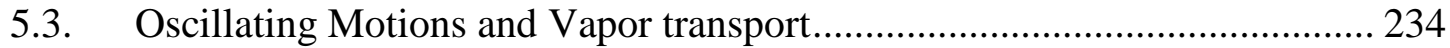

5.4 Two-Phase Heat Transfer with Oscillating Flow.......................................... 241 
5.4.1 Heat Transfer Mechanisms in Two-Phase Operation of the RMDHL ..... 242

CHAPTER SIX: CONCLUSIONS............................................................................ 245

REFERENCES ............................................................................................................ 249

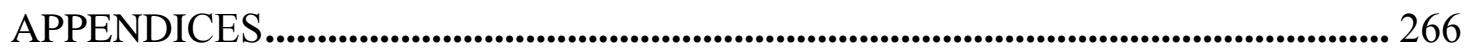

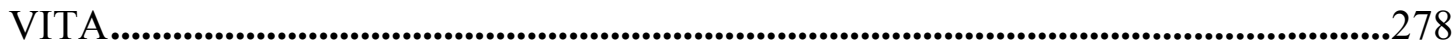




\section{LIST OF TABLES}

TABLE.

PAGE

Table 3. 1: Summary of input variations for experimental investigation into nu correlation 95

Table 3. 2: Experimental grid for single phase characterization of rmdhl. 95

Table 3. 3: Calculation of single phase thermal conductance based on experiment with tc $=45^{\circ} \mathrm{c}$ 101

Table 4.1: Dimensions, geometries and boundary conditions for the present cooling loop 148

Table 4. 2: Results for statistical and ranking for turbulent prediction methods ...... 170

Table 4. 3: Boundary conditions for the resent simulation 175

Table 4.4: Dimensions, geometries and boundary conditions for the present cooling loop simulation. 175

Table 4.5: Details of the numerical solution variables. 176

Table 4. 6: Mesh information for numerical investigation. 180

Table 4. 7: Bulk temperature variation across various cross-sectional areas of the hot plate.

Table 4. 8: Standard deviation of temperature across cross-sections of the hot plate. 190

Table 4. 9: The boundary conditions for the governing equations 193

Table 4. 10: Betails of the numerical solution variables 194 


\section{LIST OF FIGURES}

FIGURE

PAGE

Figure 1. 1: Schematic of a solenoid-operated RMDHL (Cao and Gao 2008)............ 6

Figure 1. 2: A detailed view of the solenoid driver .............................................. 7

Figure 1. 3: Picture of solenoid type RMDHL .................................................... 7

Figure 1. 4: Schematic of an RMDHL based on a bellows-type driver (Cao and Gao 2003, 2008; Cao, Xu, and Gao 2013) ...................................................... 10

Figure 1. 5: A detailed description of the bellows-type driver (Cao and Gao 2003,

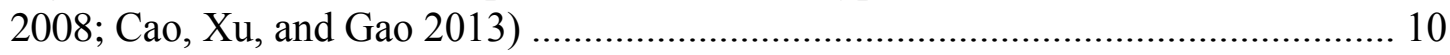

Figure 1. 6: Configurations for the bellows-type RMDHL ................................. 11

Figure 2. 1: Schematics of the battery pack design with different cell configurations (Q. Wang et al. 2012)

Figure 2. 2: Schematics and pictures of different battery configurations: (a) module level; (b) pack level (H. Liu et al. 2017)

Figure 2. 3: Schematic of a typical liquid cooling setup for PC's (Kang, Miller, and Cennamo 2007)

Figure 2. 4: Schematic of the physical model for the pulse tube refrigerator 1. compressor; 2. aftercooler; 3. regenerator; 4. cold end heat exchanger; 5. pulse tube; 6 . hot end heat exchanger; 7. orifice; 8 . reservoir; 9. double-inlet valve (Ju, Wang, and Zhou 1998) 
Figure 2. 5: Physical model of Sterling engine regenerator (Tanaka, Yamashita, and Chisaka 1990)

Figure 2. 6: Geometry and thermal boundary conditions for a periodically reversing flow in a heated tube (T. Zhao and Cheng 1995)

Figure 2. 7: The geometry and boundary conditions used in Reciprocating Flow Forced Convection (Sert and Beskok 2003) 26

Figure 2. 8: Definition sketch for Turbulent oscillatory boundary layers at high Reynolds numbers (Jensen, Sumer, and Fredsoe 1989) 27

Figure 2. 9: The geometry and boundary conditions for the RMDHL study 27

Figure 2. 10 Variation of velocity profiles in laminar oscillating pipe flow with time time during one cycle. Fluid Accelerated to the right (Ch. Walther et al. 1998)

Figure 2. 11: Pressure drop in turbulent oscillating pipe flow compared to laminar theory (C Walther, Kühl, and Schulz 2000)

Figure 2. 12: Relative phasing of cylinder volume, mass of vapor, and evaporation condensation rate(West 1982) 52

Figure 2. 13: Thermodynamic cycles for Oscillating cooling (a) P-v (b) T-S

(Woodbank Communications Ltd 2005; Tziranis 1992) 66

Figure 2. 14 : Contour plot of Nusselt Number (Su, Davidson, and Kulacki 2011) (a) Averaged for various cases (b) Contour plot of ratio of real $N u_{r} / N u_{i}$ 67 
Fig. 3. 1: First generation bellow type RMDHL for Single phase high heat rate operation(Cao, $\mathrm{Xu}$, and Gao 2013) (a) A photo of the assembled bellows-type heat loop being evacuated (b) Configuration of the designed bellows/driver assembly

Fig. 3. 2: Alternate configuration of First generation RMDHL with a condenser designed for higher cooling loads for single phase high heat flux cooling

Fig. 3. 3: Second generation bellow type RMDHL for Single phase high heat rate operation (a) A photo of the assembled second-generation bellows-type heat loop without the RMDHL driver (b) Schematic drawing of the second generation RMDHL assembly. 75

Fig. 3. 4: Photo of the Evaporator, condenser and connecting tube configuration adopted in third generation prototype

Figure 3. 5: (a) Physical dimensions of the cold plate and (b) thermocouple locations on the cold plate (size in $\mathrm{mm}$ ) (Cao, $\mathrm{Xu}$, and Gao 2013).

Figure 3.6: Battery, Module and Pack arrangement in the Tesla Model S (Shankleman et al. 2017)

Figure 3. 7: Cooling configuration for the battery module in Tesla model S electric vehicle (Steve Hanley 2016).....

Figure 3. 8: (a) Picture of Configuration of an experimental setup to characterize RMDHL for single phase correlation (b) Schematic drawing of the third generation RMDHL assembly 
Figure 3. 9: Variations in RMDHL loop for single flow characterization showing different distances between heat source and condenser (a) configuration A $(15 \mathrm{~cm})(b)$ configuration B $(30 \mathrm{~cm})(\mathrm{c})$ configuration $\mathrm{C}(60 \mathrm{~cm}) . .86$

Figure 3. 10: Overall arrangement of Single Phase Experimental Setup (Cao, $\mathrm{Xu}$, and Gao 2013)

Figure 3. 11: Experimental setup for two-phase testing Bellow type 90

Figure 3. 12: A photograph of the fabricated reciprocating-mechanism driven heat loop.

Figure 3. 13: Isometric view of the redesigned 2 phase loop 93

Figure 3. 14: $\mathrm{Q}$ vs. $\Delta \mathrm{T}$ at $\omega=1 \mathrm{rps}$, and $\mathrm{L}_{\mathrm{EC}}=30 \mathrm{~cm}$ 98

Figure 3. 15: $\mathrm{Q}$ vs. $\Delta \mathrm{T}$ at $\omega=2 \mathrm{rps}$, and $\mathrm{L}_{\mathrm{EC}}=60 \mathrm{~cm}$ 99

Figure 3. 16: $\mathrm{Q}$ vs $\Delta \mathrm{T}$ at $\mathrm{A}=7.62$ and $\mathrm{L}_{\mathrm{EC}}=60 \mathrm{~cm}$

Figure 3. 17: $\mathrm{Q}$ vs $\Delta \mathrm{T}$ at $\omega=2 \mathrm{rps}$ and Amplitude $=7.62 \mathrm{~cm}$. 99

Figure 3. 18: Comparison of experimental $k_{e f f}$ with correlations from Shin Shin \& Nishio (1998) 101

Figure 3. 19: Comparison of experimental heat transfer coefficient with various correlations at $\mathrm{f}=1 \mathrm{~Hz}$.

Figure 3. 20: Comparison of experimental heat transfer coefficient with various correlations at $\mathrm{f}=2 \mathrm{~Hz}$.

Figure 3. 21: Effective heat transfer coefficient against stroke for RMDHL ........... 105

Figure 3. 22: Heat transfer coeffcient against stroke for RMDHL .......................... 106 
Figure 4. 1: Physical model of Sterling (Tanaka, Yamashita, and Chisaka 1990). .109

Figure 4. 2: Schematic diagram of the Alpha-type Stirling engine cooler (Kuosa et al. 2012) 109

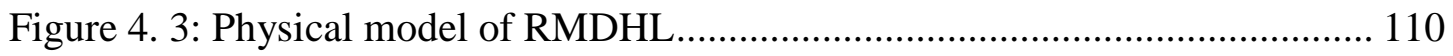

Figure 4. 4: External boundary conditions for this study.................................. 112

Figure 4. 5: Schematic of the distribution of wall heat flux in the two phase model

Figure 4. 6: Illustration of how how the RPI model was extended for two phase analysis of reciprocating two phase flow using quenching heat flux ( $q Q$ ) and case study 116

Figure 4. 7: CAD developed for first generation bellows type RMDHL

Figure 4. 8: CAD developed for second generation Bellows Type RMDHL

Figure 4. 9: CAD developed for third generation bellows type RMDHL

Figure 4. 10: Geometrical Model of Bellows - type RMDHL indicating one of the bellows and suppressed solid sections

Figure 4. 11: Meshes of the geometrical model of the right bellows of the RMDHL 136

Figure 4. 12: (a) Geometry of the RMDH loop used for numerical model (b) Grid distribution for the RMDH loop 139

Figure 4. 13: Velocity profile for (a) pulsating viscous flow (Uchida 1956), (b) pulsating viscous flow of this study at various times 145 
Figure 4. 14: the 2D geometry of the cooling loops.

Figure 4. 15: mesh distribution for a 2D

Figure 4. 16: Temperature contour for the evaporator, condenser and solid walls.

Figure 4. 17: Pressure contour for the condenser loop.

Figure 4. 18: Pressure contour for the evaporator loop.

Figure 4. 19: Velocity vectors of the condenser loop.

Figure 4. 20: Velocity vectors of the evaporator loop.

Figure 4. 21: Temperature profiles on the evaporator walls for $\mathrm{V}_{\max }=0.01 \mathrm{~m} / \mathrm{s}$, $\mathrm{q} '=2000 \mathrm{~W} / \mathrm{m}^{2}$ and $\mathrm{T}_{\text {condensor }}=283 \mathrm{~K}$.

Figure 4. 22: Temperature profiles on the evaporator walls for $\mathrm{V}_{\max }=0.01$ $\mathrm{m} / \mathrm{s}, \mathrm{q}$ ' $=5000 \mathrm{~W} / \mathrm{m}^{2}$ and $\mathrm{T}_{\text {condensor }}=283 \mathrm{~K}$.

Figure 4. 23: temperature profiles on the evaporator walls for $\mathrm{V}_{\max }=$ $0.01 \mathrm{~m} / \mathrm{s}, \mathrm{q}$ ' $=10000 \mathrm{~W} / \mathrm{m}^{2}$ and $\mathrm{T}_{\text {condensor }}=283 \mathrm{~K}$

Figure 4. 24: temperature profiles on the evaporator walls for $\mathrm{V}_{\max }=0.03 \mathrm{~m} / \mathrm{s}$, q"' $=2000 \mathrm{~W} / \mathrm{m}^{2}$ and $\mathrm{T}_{\text {condensor }}=283 \mathrm{~K}$ 155

Figure 4. 25: temperature profiles on the evaporator walls for $V_{\max }=0.03$ $0.03 \mathrm{~m} / 2, \mathrm{q}^{\prime \prime}=5000 \mathrm{~W} / \mathrm{m}^{2}$ and $\mathrm{T}_{\text {condensor }}=283 \mathrm{~K}$ 155

Figure 4. 26: Temperature contour on the computational domain 156

Figure 4. 27: Pressure contour on the computational domain. 156

Figure 4. 28: Temperature profile on the evaporator surface, $t=15 \mathrm{~s}, \mathrm{q}$ ' $=20000$ $\mathrm{W} / \mathrm{m} 2$. 
Figure 4. 29: Temperature profile on the evaporator surface, $T=20 \mathrm{~s}, \mathrm{q}$ ' $=20000$

$\mathrm{W} / \mathrm{m} 2$

Figure 4. 30: Temperature profile on the evaporator surface, $T=30 \mathrm{~s}, \mathrm{q}$ ' $=20000$

$\mathrm{W} / \mathrm{m} 2$

Figure 4. 31: Temperature contours of RMDHL assembly model with various turbulence prediction models.

Figure 4. 32: Temperature distributions over the cold plate for experimental and the numerical models for (a) $8.8 \mathrm{KW} / \mathrm{m}^{2}$ (b) $6.7 \mathrm{KW} / \mathrm{m}^{2}$

Figure 4. 33: The y-intercept of the best-fit regression temperature distributions over the cold plate

Figure 4. 34: Comparison of numerical and experimental Temperature distributions over the cold plate at different heat inputs for a coolant inlet temperature of $10 \mathrm{oC}$

Figure 4. 35: The contour of varying temperature across evaporator for varying heat transfer rates.

Figure 4. 36: Temperature contours of hot plate for various heat transfer rate and cooling methods

Figure 4. 37: The plot of Heat transfer rate vs. average temperature.

Figure 4. 38: The Plot of Heat transfer coefficients for both RMDHL and

DPDHL at different heat transfer rate.

Figure 4. 39: Temperature contours of hot plate for various heat transfer rate and cooling methods 
Figure 4. 40: Volume rendering of a cross-section of the hot plate showing cross section for temperature investigation.

Figure 4. 41: Temperature profile for a cross-section along hot plate (a) section A-A(b) section B-B (c) section C-C

Figure 4. 42: Temperature variation across various cross-sectional areas of the hot plate.

Figure 4. 43: Plot of Heat transfer rate and standard deviation 190

Figure 4. 44: The geometry and boundary conditions for this study

Figure 4. 45: (a) Open loop geometry configuration for numerical model of the RMDHL, (b) location of the planes for data analysis of the RMDHL, and (c) Grid distribution for numerical simulations of the RMDHL 196

Figure 4. 46: Contour of vapor volume fraction in the evaporator at (a) $212.05 \mathrm{~s}$ (b) $212.15 \mathrm{~s}$ (c) $212.35 \mathrm{~s}$ (d) $212.50 \mathrm{~s}$ (e) $213.05 \mathrm{~s}$ (f) $212.35 \mathrm{~s}$ (g) $213.50 \mathrm{~s}$

(h) $214.05 \mathrm{~s}$ (i) 214 198

Figure 4. 47: Comparison of numerical and experimental temperature distributions along the heat loop at different heat inputs for a coolant inlet temperature of $313 \mathrm{oC}$

Figure 4.48: Comparison of numerical and experimental temperature distributions along the heat loop at different heat inputs for a coolant inlet temperature of $338 \mathrm{oC}$. 
Figure 4. 49: Temperature contours of the working fluid for (a) $313 \mathrm{~K}$ condenser with $166 \mathrm{~W}$ at $715.4 \mathrm{~s}$, (b) $338 \mathrm{~K}$ condenser with $162 \mathrm{~W}$ at $474.8 \mathrm{~s}$, and (c) $323 \mathrm{~K}$

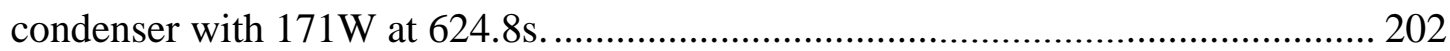

Figure 4.50: Plot of fluid temperature vs. distance from the heater location. 203

Figure 4.51: Velocity profile of cooling liquid in the evaporator over Plane 2 with $313 \mathrm{~K}$ Condenser and: (a) $166 \mathrm{~W}$ at $474.8 \mathrm{~s}$, (b) $166 \mathrm{~W}$ at $624.8 \mathrm{~s}$, and (c) $166 \mathrm{~W}$ at $715.4 \mathrm{~s}$

Figure 4.52: Temperature contours of the heat loop showing surface temperature uniformity for $323 \mathrm{~K}$ condenser inlet temperature with heat flux at (a) $75.9 \mathrm{~W}$, (b) $126.9 \mathrm{~W}$, (c) $156 \mathrm{~W}$, and (d) $171 \mathrm{~W}$ 206

Figure 4.53: Regression analysis for the SD of the of the cold plate static temperature for operating conditions ...... 206

Figure 4. 54: Plot of Inlet volume flow rate against iteration 209

Figure 4. 55: Plot of Outlet volume flow rate against iteration 209

Figure 4. 56: Plot to compare the difference between inlet and out volume flow rate for half cycle of RMDHL driver to reflect variation in volume flow due to evaporation and condensation. 210

Figure 4. 57: Plot of Volume integral of pressure in the evaporator for one reciprocating cycle of the RMDHL driver

Figure 4. 58: P-V Diagram for RMDHL

Figure 4. 59: Flow chart for developing P-V diagram from Numerical model 212

Figure 4. 60: Temperature fluctuations across hot plate at $600 \mathrm{~W} / \mathrm{cm}^{2}$ 213 
Figure 5. 1: The schematic of the heat loop (based on the solenoid type RMDHL) for the derivation of the working criterion

Figure 5.2:Schematic drawing for Estimating power consumption in RMDHL illustrating the Motion of the piston in the reservoir. 218

Figure 5.3: Schematic of pressure distributions in a RMDHL configuration........... 219

Figure 5. 4: Analysis of the volume of one vapor bubble formed during heat transfer in the RMDHL

Figure 5. 5: Schematic illustration of how the Pressure forces in the RMDHL loop are resolved 230

Figure 5. 6: Pressure distributions in the RMDHL adapted from Ma et al.(2006) ... 236 


\section{CHAPTER ONE: INTRODUCTION}

The rapid evolution of technology in the last decade is due to the sophistication and high performance of the electronic chips that power the systems and subsystems of the inventions. These inventions vary from power electronics, plasma-facing components, high heat-load optical components to laser diode arrays. Other devices include X-ray medical devices, power electronics in hybrid vehicles also for toys and appliances, high-power computers, power/optoelectronic equipment, cooling the leading edges/nose caps of supersonic/hypersonic cruise vehicles applications, advanced gas turbines, and new directed energy based weapon systems. The higher performance of these electronics chips and indeed their overall systems is accompanied one way or the other by higher heat generation (Zuo, Hoover, and Phillips 2002). Because failure rate of these devices themselves increases exponentially with the rate of increasing temperature, active thermal management of the systems are required. Thermal management and related design problems continue to be identified by the Semiconductor Industries Association Roadmap (Semiconductor Industry Association 1999; Z. Liu et al. 2014) as one of the five critical challenges during the next decade to achieve the projected performance goals of the industry. 
Finding effective thermal solutions has become a major constraint for the reduction of cost and time-to-market, two governing factors between success and failure in commercial evolution of technology (Zuo, Hoover, and Phillips 2002). In addition to the time urgency, the heat generation problem is further compounded by the increasing trend of miniaturization of these chips. It has been observed over the years that these chips have gotten smaller and more compact, and the smaller they get the higher the heat flux associated with their operation. Microelectronic chips may dissipate heat as high as $10 \mathrm{~W}$ through a $5 \mathrm{~mm} \times 5 \mathrm{~mm}$ area, reaching a heat flux of 400W/cm² (Vafai 2002; Bergles 2003; Z. Liu et al. 2014) and heat fluxes over 1,000 W/ $\mathrm{cm}^{2}$ have been projected (Hetsroni, Mosyak, and Segal 2001). As a result there is a need to create a capability to effectively remove these high heat fluxes.

An efficient and effective thermal management system must also find a solution to non-uniform system temperature or heat flux distribution across cooled surfaces (Krishnamoorthy 2008). This statement is especially true for the cooling in cylindrical Li-ion cell pack used in the $5.3 \mathrm{kWh}$ electric vehicle (EV) to $129 \mathrm{MWh}$ massive grid energy (LGE) storage systems. The operating temperature of Lithium-ion batteries affects many aspects of a pack including round-trip energy efficiency, charge acceptance, reliability, lifetime and life cycle cost (Shabani and Biju 2015). The power pack for the EV and LGE systems is composed of a number battery modules which consist of some cells connected in series and parallel (D. Y. Kim et al. 2016). The pack that ultimately drives the $\mathrm{EV}$ is then formed by connecting multiple modules to sensors, controllers, and other components, and then the pack is housed in a custom case 
designed for the geometry specific for each application. Voltage, temperature, and other parameters are monitored continuously by sensors attached to the modules in the pack (Automotive Energy Supply Corporation 2016). Given the unique dependence of voltage supplied by the pack on operating temperature of each cell as well as the typical temperature gradient between the hot and cold feeds at each end of the heat exchanger, thermal management in these systems is a challenge.

For other high power electronics systems, even when significantly high temperatures are not attained, high thermal stresses due to temperature variations are significant causes of failure in electronic components mounted on circuit boards (Jeakins and Moizer 2003). Non-uniform heat flux distribution leads to local hot spots and elevated temperature gradients across the silicon dies, which may cause excessive thermal stresses on the device and result in ultimately device failure.

Many previously developed passive heat transfer devices, such as heat pipes and capillary pumped loops, may be inadequate for some of these cooling applications. The heat pipe and capillary pumped loop are good two-phase heat transfer devices and have found broad applications in many industries. Heat flux levels that can be handled by a heat pipe or capillary pumped loop is usually on the order of $20 \mathrm{~W} / \mathrm{cm}^{2}$ (Faghri, 1995; Cao, Xu, \& Gao, 2013)). However, heat flux requirements for some evolving technologies are in excess of $400 \mathrm{~W} / \mathrm{cm}^{2}$. An active two-phase cooling technology involving a pump is believed to be the solution for these high heat-flux cooling applications. 
A two-phase cooling system employing a traditional pump, however, could encounter the so-called cavitation problem unless the working fluid at the inlet of the pump is adequately sub-cooled (Fox and McDonald, 1998). The requirement for the pump inlet sub-cooling would inevitably increase the complexity of the cooling system and in many situations, may require a refrigeration system for the sub-cooling purpose. It should be mentioned that the provision of the sub-cooling may not represent a particular difficulty for ground cooling systems employing an open-looped configuration; but for aerospace applications additional weight required with subcooling is undesirable.

The term of two-phase heat transfer device here refers to a heat transfer system that is close-looped, compact, portable, self-contained, and able to work under a weightless condition. In this case, the coolant is circulated by a pumping device, and the boiling and evaporation are allowed to occur over the heated surface, which could provide an enhanced capability to remove a more significant amount of heat and achieve a higher level of temperature uniformity over the system. For traditional positive displacement pumps, the pumping reliability is always a serious concern. (Cao and Gao 2008). The space within the cold plate must be reserved for volume changes in the working fluid that occurs as a result of boiling and vaporization within the loop. As such, the two-phase loop cannot be completely filled with the liquid. The consequence of the partial filling of the space is that vapor or liquid-vapor two-phase mixture may enter the pump, which could cause cavitation problems and render the pump ineffective (Munson, Young, and Okiishi 2009). 
Cao and Gao (Cao and Gao 2008; Cao, Xu, and Gao 2013) conceived, designed and tested a novel Reciprocating-Mechanism Driven Heat Loops (RMDHL) or the reciprocating-flow heat loop. These devices attain a reciprocating flow of the working fluid inside the heat transfer device without requiring a reciprocating motion of the entire heat transfer device. The initial RMDHL experimental results indicated that the heat loop worked very well and could handle a heat flux of more than $300 \mathrm{~W} / \mathrm{cm}^{2}$ in the evaporator section.

Although the concept of the RMDHL has been proven, the study of this new heat transfer device thus far is very preliminary. Also, the RMDHL is only one type of the two-phase heat transfer device that utilizes reciprocating flow boiling to enhance the heat transfer; many other systems/configurations that employ the same enhance mechanism are still possible. Furthermore, the underlying heat transfer mechanism in such a heat transfer mode is mostly unclear. In the two-phase operation mode of the RMDHL, the flow is reciprocating, turbulent, and two-phase. The combination of these three conditions represents a new heat transfer phenomenon that had never been studied before. As a result, a fundamental study of this unique fluid flow and heat transfer is needed for advancing the scientific understanding of this complex flow structure and for predicting/controlling the heat transfer. The scientific understanding of this unique fluid flow and heat transfer structure would have significant archival value and would be useful for many practical applications of the RMDHL. 


\subsection{Background and Theory}

A reciprocating-mechanism driven heat loop (RMDHL) employing a solenoidoperated electromagnetic driver, as illustrated in Fig. 1.1 to Fig 1.3, comprises a hollow loop having an interior flow passage, an amount of working fluid filled within the loop, and a solenoid-operated reciprocating driver. The hollow loop has an evaporator section, a condenser section, and a liquid reservoir. Like a heat pipe, the heat loop is air evacuated and hermetically sealed to enhance the two-phase heat transfer process. A solenoid reciprocating driver (Fig. 1. 2) is integrated with the liquid reservoir and facilitates a reciprocating flow of the working fluid within the loop so that liquid is supplied from the condenser section to the evaporator section under a substantially saturated condition and the so-called cavitation problem associated with conventional pumps is avoided (Fig. 1.3).

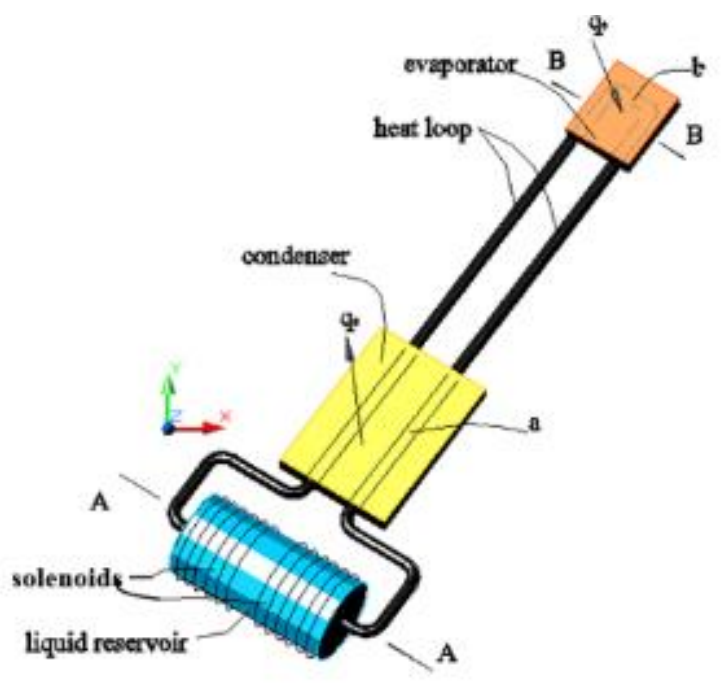

Figure 1. 1: Schematic of a solenoid-operated RMDHL (Cao and Gao 2008) 


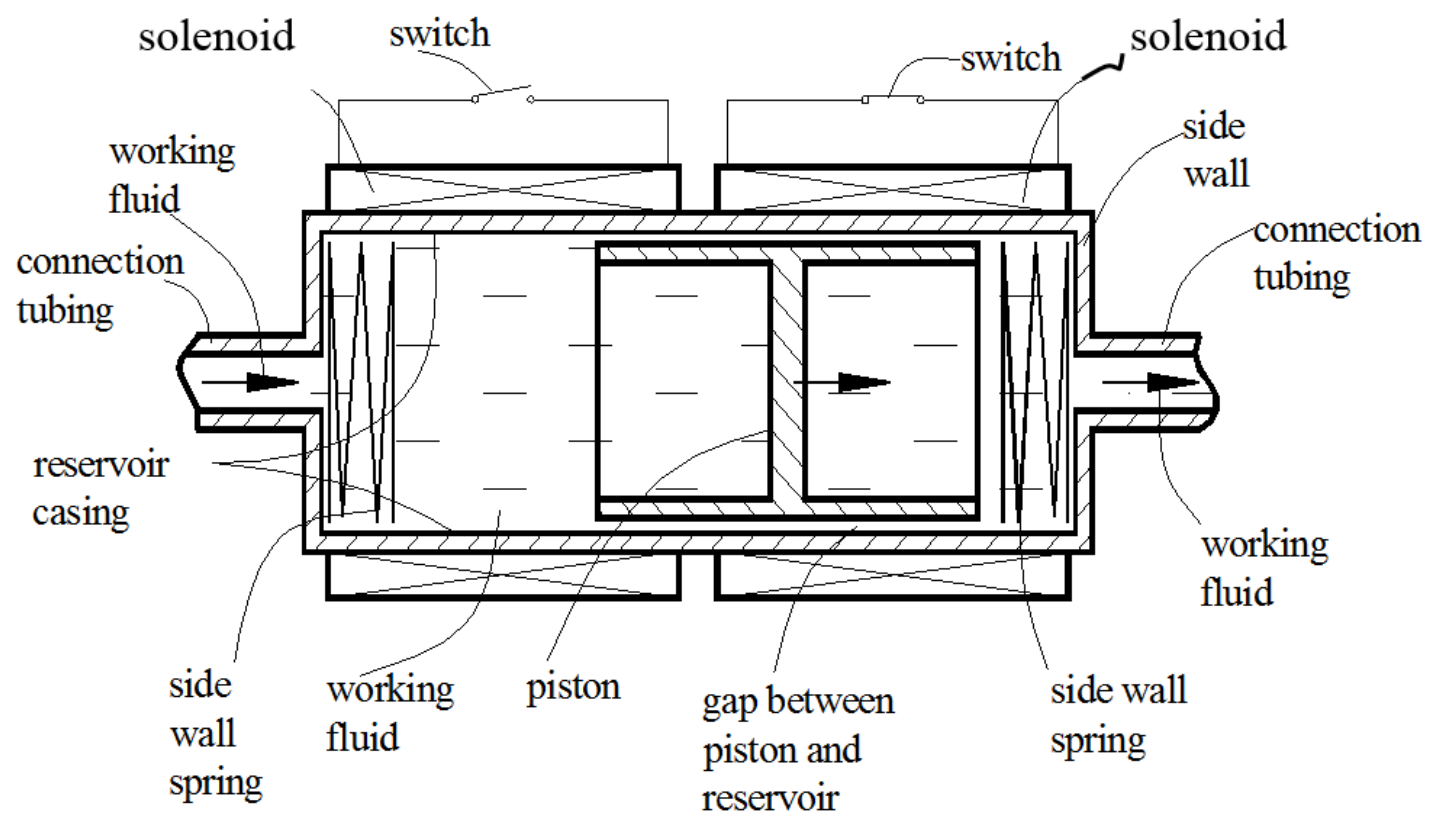

Figure 1. 2: A detailed view of the solenoid driver

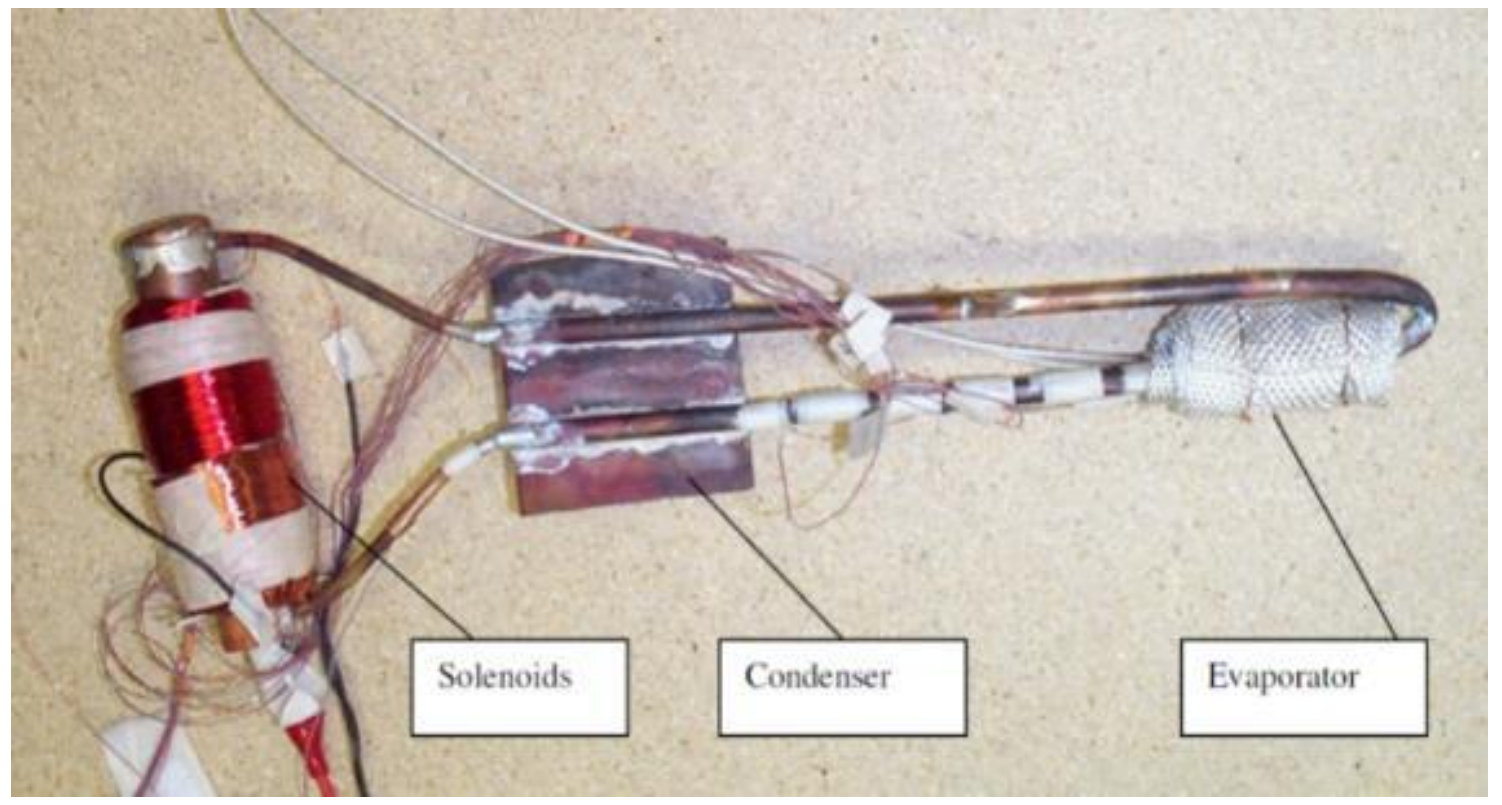

Figure 1. 3: Picture of solenoid type RMDHL 
In the case shown in Fig. 1.2, the circuit of the right-hand solenoid is closed through a switch while the circuit of the left-hand solenoid is opened. As a result, the piston is being attracted towards the right through the magnetic field generated by the solenoid, and a counterclockwise flow of the working fluid within the loop is produced. When the piston approaches the right end of the liquid reservoir, the switch of the lefthand solenoid is closed while the switch of the right-hand solenoid is opened. As a result, the piston stops being attracted by the right-hand solenoid and is attracted by left-hand solenoid towards the left, and a clockwise flow of the working fluid within the loop is produced (not shown here).

With the circuits of the two solenoids being opened and closed alternating of each other, a reciprocating motion of the piston is induced. This motion, in turn, produces a reciprocating flow of the working fluid within the heat loop. With a sufficiently large piston stroke, the liquid is efficiently supplied to the evaporator section from the condenser section. Unlike a conventional pump, a unidirectional pressure head across the pump is not necessary. In fact, the pressure at a given location of the loop would oscillate around a particular value. The working fluid in the loop is driven from the condenser section to the evaporator section reciprocating through the displacement of the partition. As a result, the century-old cavitation problem associated with a conventional pump is avoided.

When the working temperature of the heat loop is high, a bellows-type reciprocating heat loop employing an external reciprocating mechanism could be used (Fig. 1.4 - Fig 1.6). As shown in Fig. 1.4, through a connecting rod, the bellows are 
coupled to an external reciprocating mechanism which could produce a reciprocating motion with a sufficiently sizeable reciprocating stroke. The external reciprocating mechanism can be a solenoid-operated electromagnetic driver or a mechanical reciprocating mechanism driven by an electric motor. Since the bellows-type reciprocating heat loop does not contain any contacting surfaces having a relative motion in the high-temperature region, it could work at a much higher temperature. Additionally, during the operation of the bellows-type driver, the outer surface of the bellows can be adequately cooled so that the bellows can be maintained at a sufficiently low temperature for its reliability.

Experimental studies have been undertaken to validate the proposed (Cao and Gao 2003, 2008; Cao, Xu, and Gao 2013) concept. The results showed that the heat loop worked very efficiently with an effective thermal conductance more than 120 times that of copper. The experiments also showed that a heat flux as high as 300 $\mathrm{W} / \mathrm{cm}^{2}$ based on the contact surface area between the heater and the outer loop surface in the evaporator section could be handled. The experimental data also demonstrated that at moderate driver's reciprocating frequency of $0.5-1.0 \mathrm{~Hz}$, the temperature at the heated surface was remarkably stable without noticeable fluctuation. The experiments set up for the studies by Cao et al. (2003 and 2011) used a $100 \mathrm{~W}$ electric heater for a contact surface area between the heater and the heat loop being approximately 0.56 $\mathrm{cm}^{2}$. 


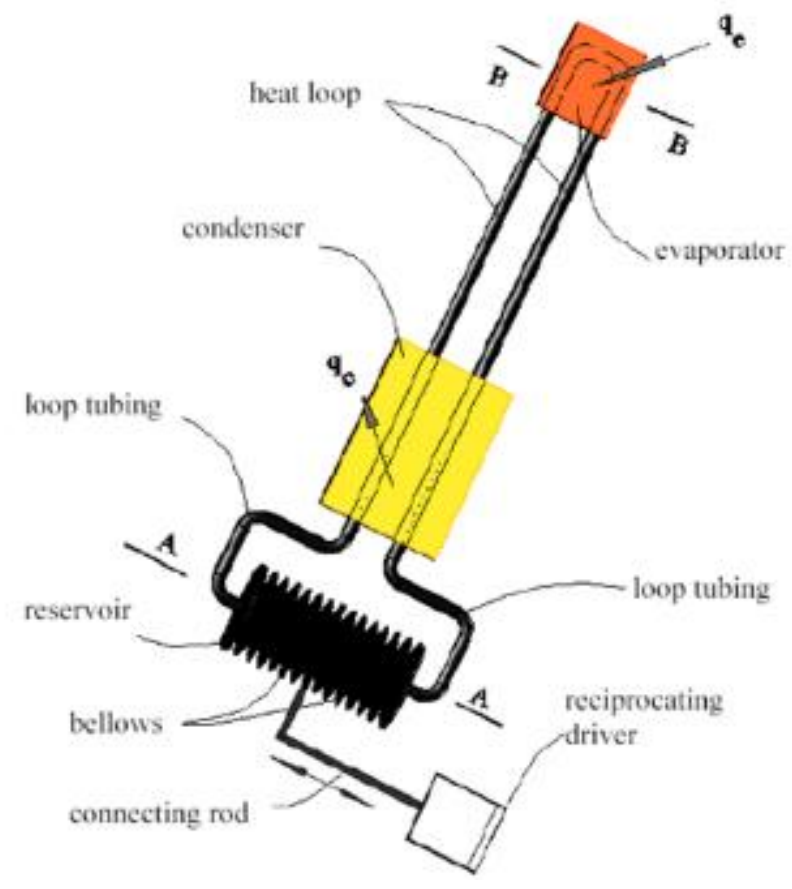

Figure 1. 4: Schematic of an RMDHL based on a bellows-type driver (Cao and Gao 2003, 2008; Cao, Xu, and Gao 2013)

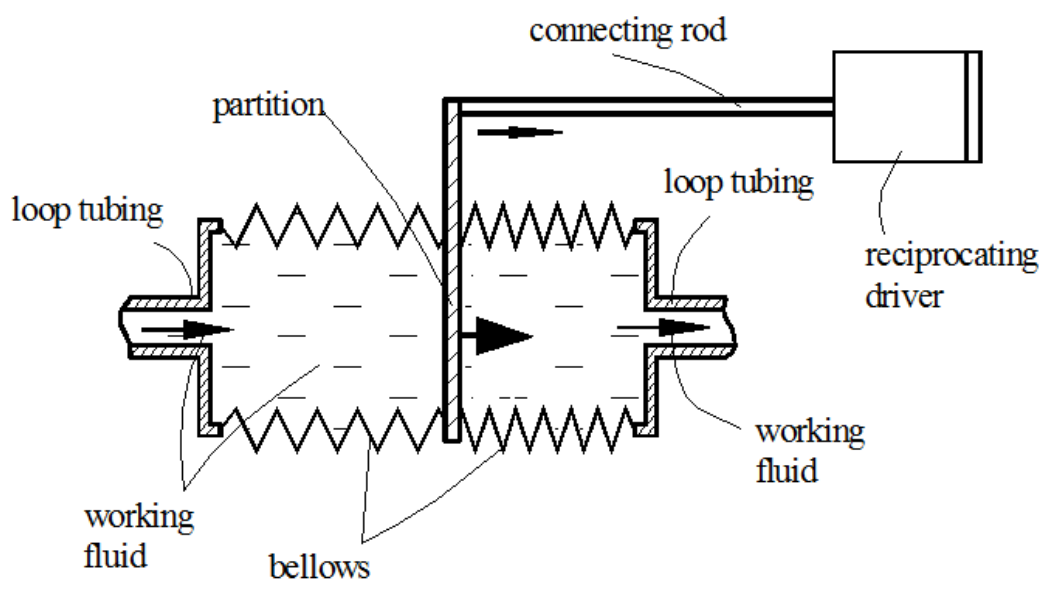

Figure 1. 5: A detailed description of the bellows-type driver (Cao and Gao 2003, 2008; Cao, Xu, and Gao 2013) 


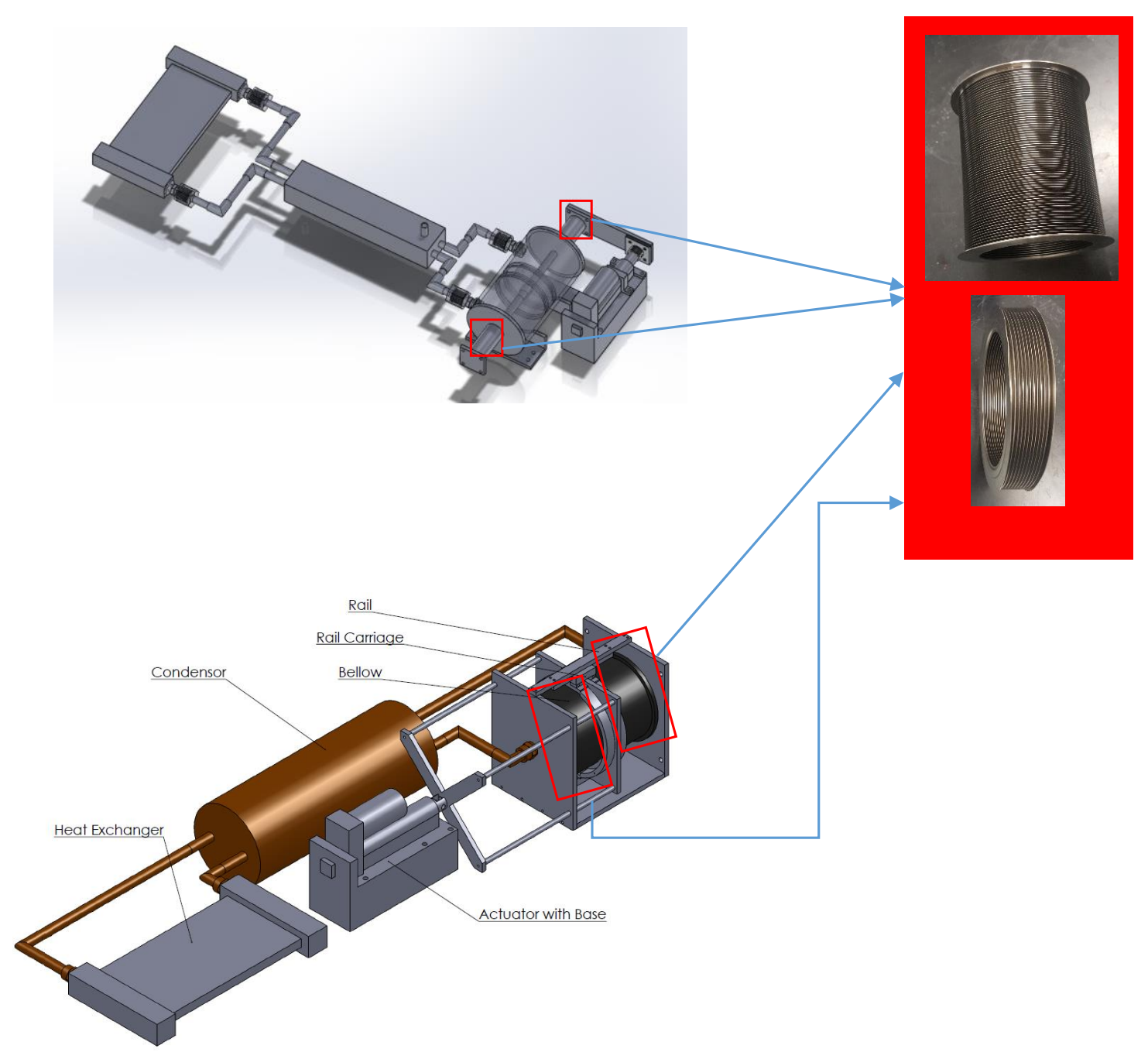

Figure 1. 6: Configurations for the bellows-type RMDHL 


\subsection{Reciprocating Heat Transfer in the RMDHL}

One of the most important heat transfer mechanisms of the RMDHL operation is believed to be the boundary layer development/destruction cycle in conjunction with the fluid mixing under a reciprocating condition (Simon and Seume 1988). It is commonly known that a boundary layer would be developed as fluid flows in a given direction, e.g., clockwise (Bergman, Lavine, and Incropera 2012). As the fluid reverses the direction and flows counterclockwise, the boundary layer developed earlier will be destroyed and a new boundary layer will be developed. This boundary development/destruction cycle will be controlled by the reciprocating frequency of the reciprocating driver. It is expected that as the reciprocating frequency is increased to a certain value, the time-averaged boundary layer will be so thin that the heat transfer coefficient associated with the reciprocating flow would be much higher than that under many other heat transfer modes. It is also expected that additional underlying heat transfer mechanisms related to the present study would exist, which could significantly improve the heat transfer compared to the single-phase reciprocating flow.

Compared to research work on other active cooling systems, research into internal oscillating flow systems is limited. Substantial research has been undertaken in recent years for single-phase reciprocating flow heat transfer to address these shortcomings. Examples of these research efforts include those of Cooper et al. (1994), Zhao and Cheng, (1995), Li and Yang (2000), and Sert and Beskok (2003). However, these mainly use air as the working fluid for air cooling enhancement. $\mathrm{Li}(\mathrm{P} . \mathrm{Li}$ and Yang 2000) provided insight into the mechanism for improved heat transfer in 
oscillating flow over conventional flow. Implications of the so-called annular effect where the maximum axial velocity in some oscillatory flow occurs near the wall were first observed by Richardson and Tyler (Richardson and Tyler 1929; Kuosa et al. 2012; Xiao, Zhou, et al. 2014).

More significant for inventors and designers of thermal management systems among these research efforts is the work to improve the accuracy of the analytical correlations associated with quantifying system fluid mechanics and thermal variables. It was observed in the literature that correlations for heat transfer in oscillating flow conditions are usually based on correlations derived from unidirectional flow assumptions (Kuosa et al. 2012). A few other correlations were developed from approximate analytical and numerical solution of the Naiver-Stokes equation and experimental measurements. Specific dissimilarities between internal oscillating flow and conventional flow have been itemized, and the shortfalls in the correlations have been highlighted (Allan 2014). While in a few situations some of these correlations provide a close approximation, the shortfall in many other predictions is as much as $15 \%$ to $25 \%$, beyond acceptable limits.

\section{$1.3 \quad$ Objectives}

An objective of the current research is to provide a further validation of the concept of the bellows-type RMDHL with an emphasis on the achievement of substantial temperature uniformity over a cold plate. This study will also develop a suitable numerical model for the RMDHL which up until now is unavailable. The 
numerical model may help avoid costly redesign and allow the testing and possible incorporation of new thermofluid assumptions into the RMDHL, which will help achieve our aim. This study will produce significant experimental and numerical data on the limits of the design of the RMDHL, which may include fatigue studies, device scaling for the diversity of applications, and relevant parametric analysis of the device to help designers select the best solution.

The specific objectives of this research are:

1 Understand the underlying physical mechanisms of boiling heat transfer in internal reciprocating flow.

2 Conduct an experimental study of the heat transfer characteristics of the RMHL and obtain experimental data on the effect of the operating parameters.

3 Develop a comprehensive numerical model for the RMDHL that will be validated by the experimental results.

4 Produce practically useful correlations based on the numerical and experimental data for future RMDHL designs.

This research provides numerical and experimental data for the RMDHL and generates semi-empirical correlations, all of which will provide archival information for the design of a two-phase heat transfer device involving a reciprocating flow. In particular, this research will lead to the development of more powerful reciprocatingmechanism driven heat loops (RMDHLs), achieve a heat flux goal of $600 \mathrm{~W} / \mathrm{cm}^{2}$, and significantly advance the thermal management at various levels. The developed two- 
phase heat transfer device could become an enabling technology for the thermal management of high-power electronics, cooling the leading edges/nose caps of supersonic/hypersonic cruise vehicles, removing the waste heat for directed energy based new weapon systems, and cooling advanced gas turbines.

In the present study, it is believed that both the heat transfer coefficient may be affected by the piston stroke of the reciprocating driver, piston reciprocating frequency, liquid filling ratio, size of the heat loop including the inner diameter of the liquid reservoir and the diameter of the loop tubing, working fluid of the loop, and orientation of the loop relative to the gravitational field. These essential parameters will be systematically varied in this study for broad applications. 


\section{CHAPTER TWO: LITERATURE REVIEW}

\subsection{Emerging Frontiers for the RMDHL}

\subsubsection{Battery Pack cooling (temperature uniformity)}

The battery arrangements for the EV and LGE systems composed of a number battery modules which consists of many cells connected in series and parallel (D. Y. Kim et al. 2016). The pack which ultimately drives the EV and LGE systems are formed by connecting multiple modules as shown in Fig. 2.1 and Fig. 2. 2. Individual cells generate heat from the following sources:

1. Internal heat generation due to electrochemical reactions.

2. Resistance or Joules effect heating is known as $I^{2} R$ heating $(I=$ current and $R=$ internal resistance).

3. External heating or cooling from the surroundings, such as conduction, convection and/or radiation, will also change the temperature.

A single battery pack for an EV like the one in Tesla model S contains about 7000 cells while the LGE system consists of millions of cells, and quite a lot of heat is generated in these clusters. Battery thermal management system (BTMS) have been primarily including the air-based thermal management system, the liquid-based thermal management system, and the phase change material (PCM) thermal management 
system as well as a few other technologies (Rao and Wang 2011; Yang et al. 2016). The conventional battery cooling systems utilize the unidirectional coolant flow from the inlet and outlet of the battery cooling systems. As a result of the convective heat transfer along the flow stream, the fluid temperature at the outlet is always higher than the inlet, and the downstream cells near to the outlet are likely hotter than the upstream cells.

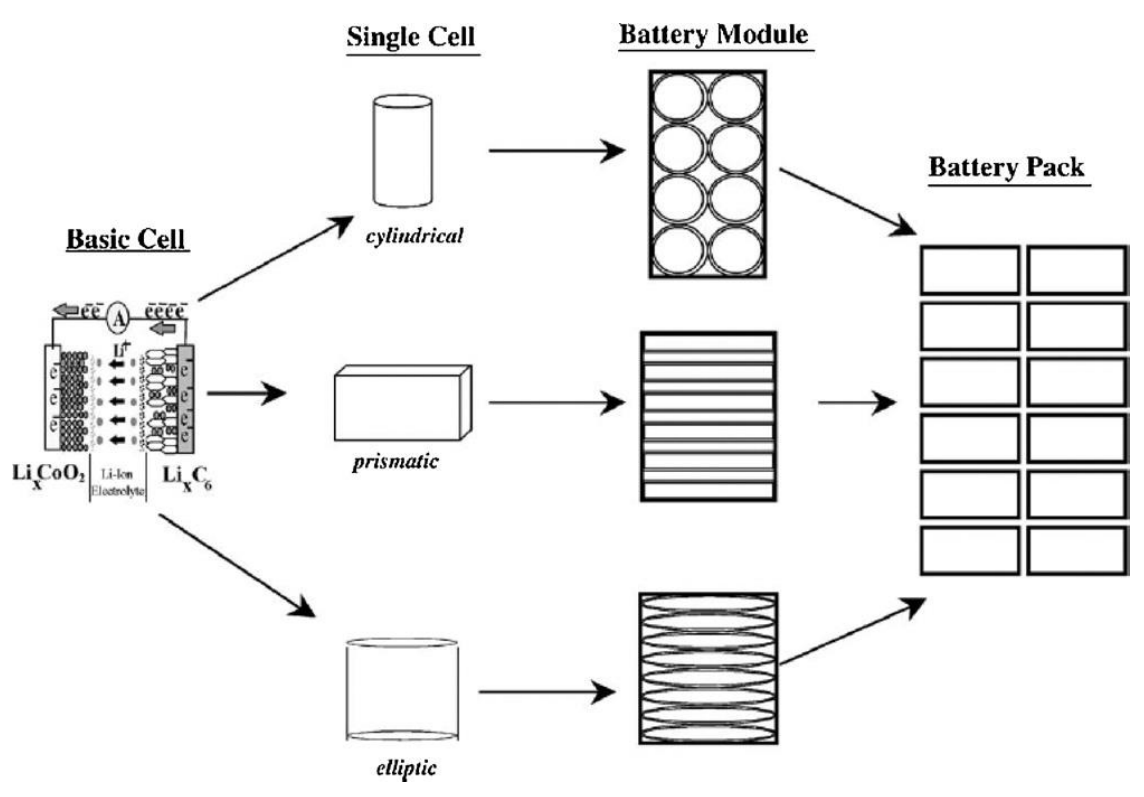

Figure 2. 1: Schematics of the battery pack design with different cell configurations (Q. Wang et al. 2012) 

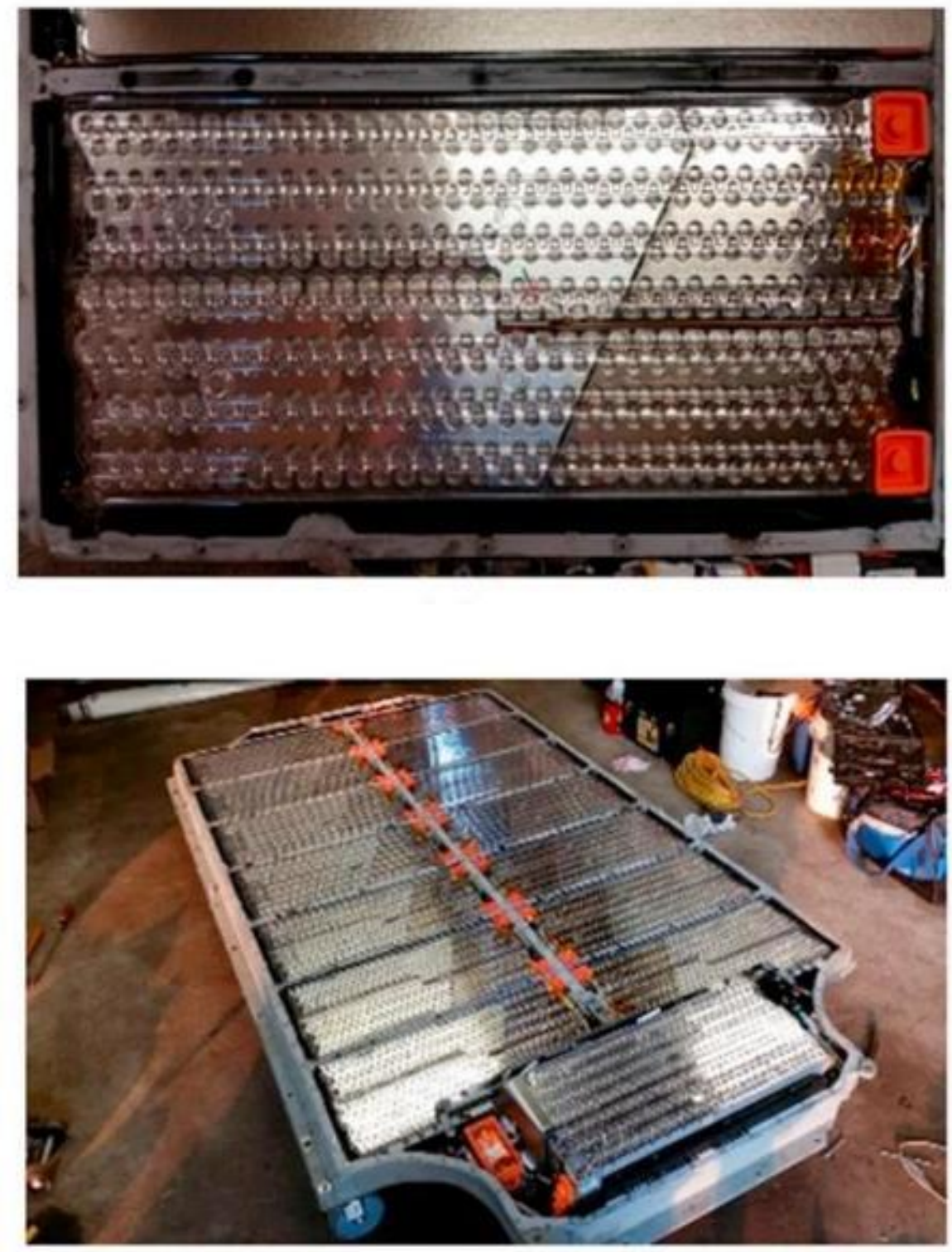

Figure 2. 2: Schematics and pictures of different battery configurations: (a) module level; (b) pack level (H. Liu et al. 2017) 
Depending on the geometry, material or construction, location of positive and negative terminals and the thermal management system, there is a non-uniform temperature distribution in a pack (Mahamud and Park 2011). In general, temperature gradients affect the following battery performance:

(i) Electrochemical system.

(ii) Round trip efficiency.

(iii) Charge acceptance.

(iv) Power and energy capability.

(v) Reliability.

(vi) Cycle life and cost.

It is desirable to have a temperature non-uniformity of $<5{ }^{\circ} \mathrm{C}$ in the thousands of cells in the pack (Shabani and Biju 2015). In order to achieve the optimum performance from a battery, a new thermal management system is required to regulate the batteries to operate in the desired temperature range and reduce uneven temperature distribution.

The RMDHL is a best candidate for the new battery cooling systems, as both experimental data and numerical simulation of the RMDHL reveals that it offer superior temperature uniformity when compared to conventional water cooling loop (O. Popoola, Soleimanikutanaei, and Cao 2016b, 2016a). 


\subsubsection{Liquid cooling system employing a RMDHL with leakage free}

Typically, computing processor power is specified in Thermal Design Power (TDP) (Scott Huck 2011). The thermal design power is defined as the maximum power a processor can draw for a thermally significant period while running commercially useful software, and many computer chips have recently crossed the $300 \mathrm{~W}$ line. The most used method of thermal management for computer systems remains air cooling because of low cost, smooth implementation, and reliability.

However, to achieve the required cooling, more massive heat sinks and increased heat sink airflow is required. This approach can lead to mechanical stress resulting from the large, massive heat sinks and intolerable noise levels (Akselband, Whitenack, and Goldman 2006). As a result more applications are turning to liquid cooling that comprises of a mechanically pumped single-phase liquid coolant, a cold plate where the heat is absorbed by the coolant (Agostini et al. 2007), a series of coolant-filled tubes, a radiator, water blocks (the equivalent of heat sinks), a liquid reservoir, a pump and related motor, and a fan. Most of the cooling water systems employ a rotodynamic pump to transport the cooling fluid around the cooling loop. To achieve temperature uniformity, a specially designed cold plate is used for most commercially available LCS, as shown in Fig. 2.3. However, the conventional liquid cooling system as mentioned above is prone to liquid leakage and the inlet of the pump must flooded by the liquid. The RMDHL could be an excellent alternative for the conventional LCSs because it is sealed without the concern for any coolant leakage. 


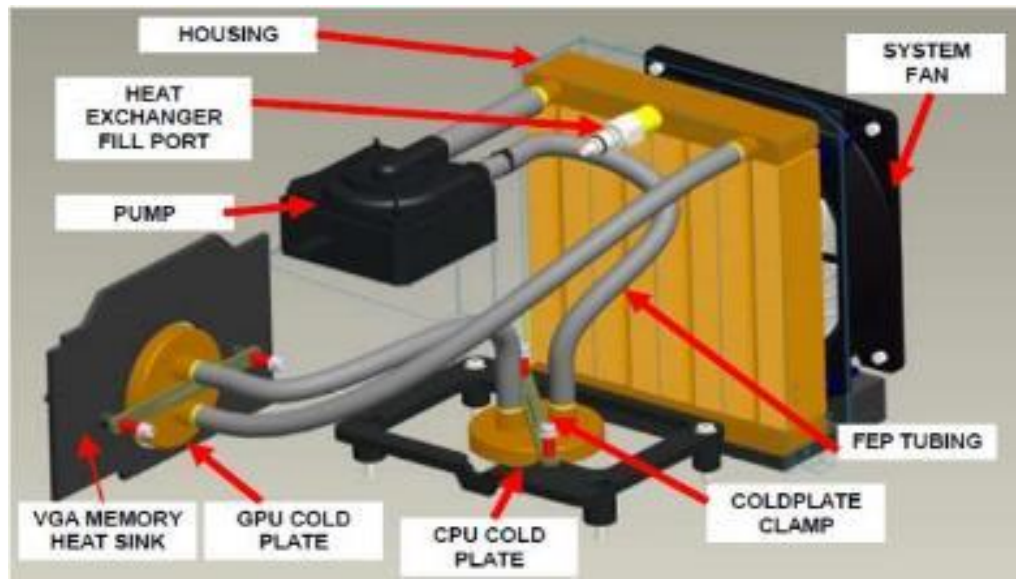

Figure 2. 3: Schematic of a typical liquid cooling setup for PC's (Kang, Miller, and Cennamo 2007) 


\subsubsection{Aerospace (Body forces, cavitation, and high heat flux application)}

In recent years, heat transfer devices that could handle a high heat flux are becoming an enabling technology for many critical aerospace applications (Sanders and Pratt, 2003), such as the National Aerospace Initiative. The National Aerospace Initiative (NAI) seeks to develop aerospace vehicles that could directly take off at ground level and travel to space. The vehicles should have the capability to operate for extended periods in a supersonic or hypersonic environment with short space/ground turn-around cycles. However, these vehicles would face tremendous challenges related to the thermal protection. During flights of hypersonic/supersonic vehicles, the stagnation regions such as the wing leading edges and nose tips could experience very high temperature, severe temperature gradients, and high stresses. Since the vehicle is required to cruise with supersonic speed, the traditional ablative thermal protection systems (TPS) could have difficulty in protecting the vehicle adequately. As a result, new thermal protection systems, including possible two-phase cooling systems, are critical to the development of these future aerospace planes.

A two-phase cooling system employing a traditional pump, however, could encounter a cavitation problem unless the working fluid at the inlet of the pump is adequately sub-cooled (Fox and McDonald, 1998). The requirement for the pump inlet sub-cooling would inevitably increase the complexity of the cooling system and in many situations, may require a refrigeration system for the sub-cooling purpose.

In a RMDHL, the reciprocating driver is integrated with the liquid reservoir and facilitates a reciprocating flow of the working fluid within the loop so that liquid is 
supplied from the condenser section to the evaporator section under a substantially saturated condition and the cavitation problem associated with a conventional pump is avoided.

\subsubsection{New weapons based on directed energy systems (very high heat flux)}

As mentioned in chapter 1, an area in which extremely high heat fluxes would be encountered is new weapons based on directed energy systems. These systems will need distributed and localized thermal management systems that require the removal of an enormous amount of waste heat generated (Sanders and Pratt, 2003). The heat flux level could be on the order of $100-1,000 \mathrm{~W} / \mathrm{cm}^{2}$ with an additional requirement for small temperature fluctuations in some situations. In this application, the RMDHL that could handle a high heat flux and maintain the system at a relatively uniform temperature is becoming an enabling technology.

\subsection{Oscillating Flow Systems and their Analytical Solutions}

Advances in thermal management systems have produced several other significant cooling solutions which adopt mechanically driven oscillating flow of working fluid within the heat loops. The flows in these devices are characterized by periodically reversing flow and heat transport between high and low-temperature reservoirs. Typical examples of these systems include pulse tube cryocooler (Fig. 2. 4), where the flow is driven by electromagnetic or thermoacoustic drivers (Hu et al. 2010) (Ju, Wang, and Zhou 1998), and Sterling engine regenerator (Fig. 2. 5) (Tanaka, 
Yamashita, and Chisaka 1990), which is applied between the high temperature reservoir and the low temperature reservoir of a Stirling engine. Although these two systems mentioned above employ a gas as the working fluid and are not directly related to the present study of the RMDHL, a large body of the knowledge, particularly for analytical solutions, had been generated by related prior studies, which could provide some significant intellectual background for the present study. For this reason, the subjects of these two studies are reviewed in this review Chapter.

A significant hindrance to the broad adoption of these two devices is posed by the fact that analytical studies of these reciprocating flow systems can be challenging. Unlike conventional unidirectional flows, mathematical description of the oscillatory flow is complicated and not easy to interpret. Particular integral and the general solutions to the homogeneous governing equations for oscillating flow, for the most part, are too complicated to provide explicit information (Chen, Luo, and Dai 2007)(Currie 2003). Depending on the physical description of the problem and the coordinate system adopted, the solution contains complex numbers, Bessel functions, and hyperbolic trig and exponential terms (Uchida 1956). In most cases, these terms require additional simplification.

It was observed in the literature that correlations for heat transfer in oscillating flow conditions are usually based on correlations derived from unidirectional flow assumptions (Kuosa et al. 2012). Some classical laminar and turbulent steady unidirectional correlations are used to get approximations of convective heat transfer coefficient in Sterling engines, as presented in (Xiao, Chen, et al. 2014). These methods 


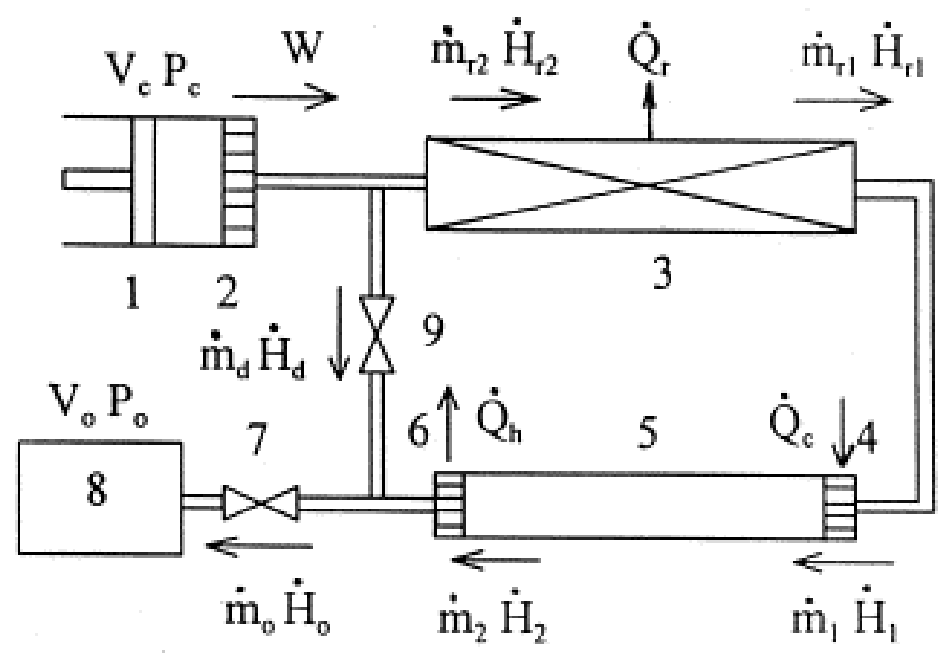

Figure 2. 4: Schematic of the physical model for the pulse tube refrigerator 1. compressor; 2 . aftercooler; 3. regenerator; 4 . cold end heat exchanger; 5 . pulse tube; 6. hot end heat exchanger; 7. orifice; 8. reservoir; 9. double-inlet valve (Ju, Wang, and Zhou 1998)

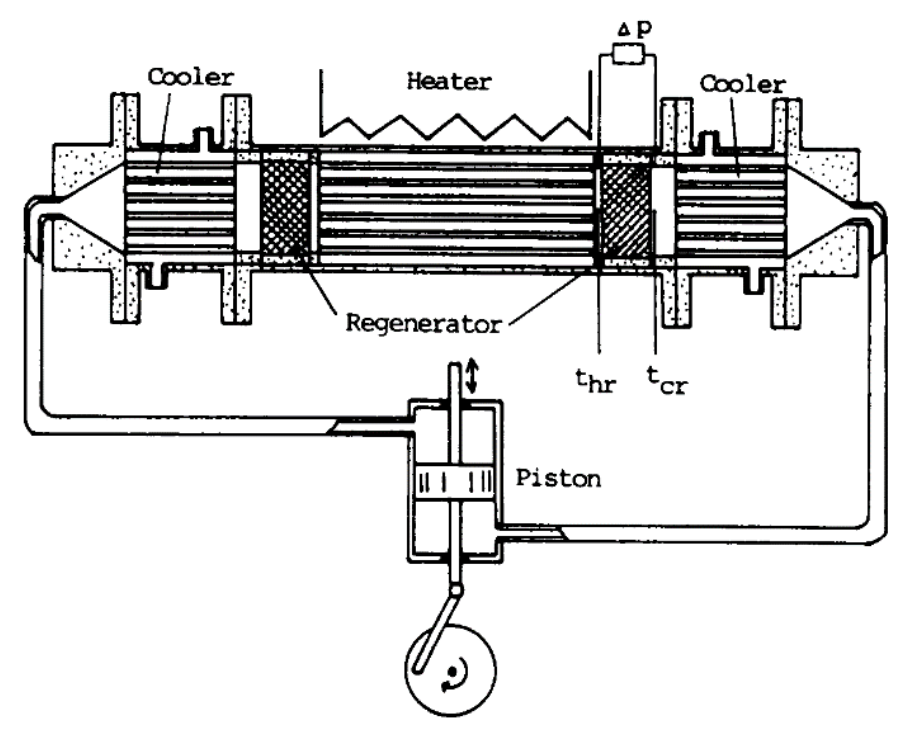

Figure 2. 5: Physical model of Sterling engine regenerator (Tanaka, Yamashita, and Chisaka 1990) 


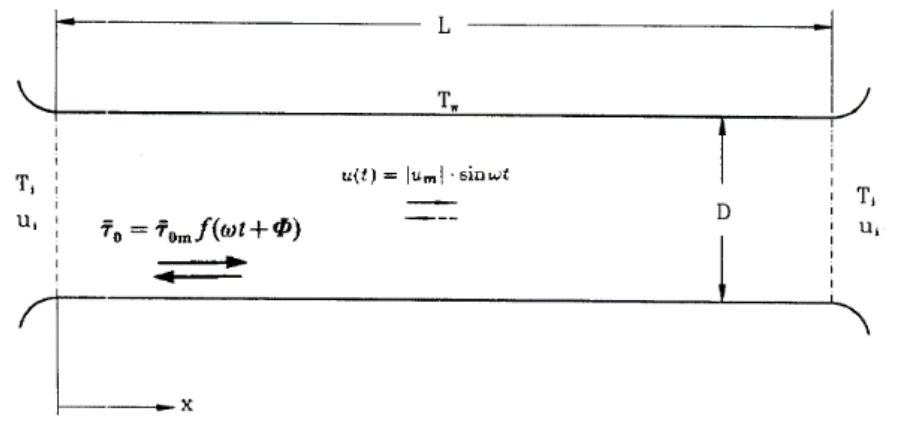

Figure 2. 6: Geometry and thermal boundary conditions for a periodically reversing flow in a heated tube (T. Zhao and Cheng 1995)

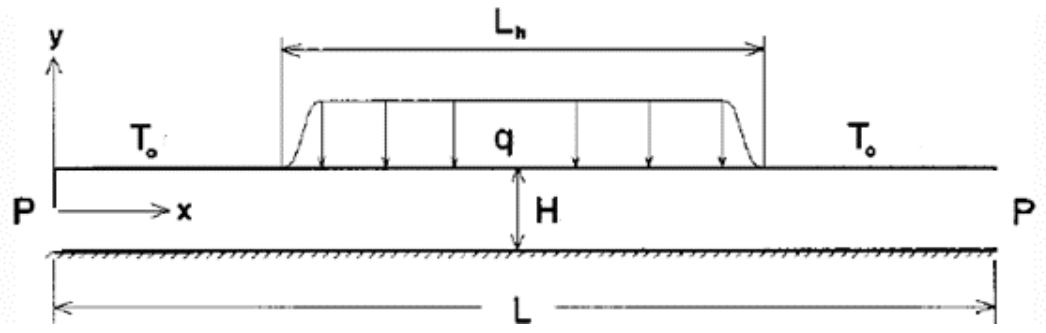

Figure 2. 7: The geometry and boundary conditions used in Reciprocating Flow Forced Convection (Sert and Beskok 2003) 


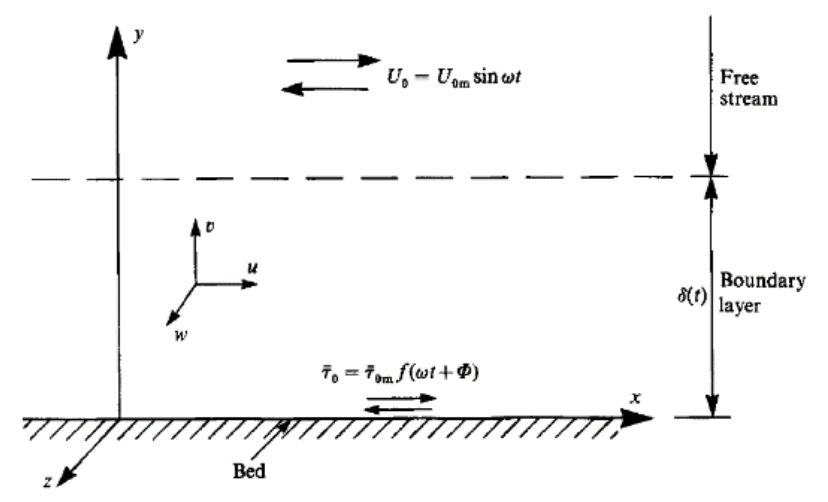

Figure 2. 8: Definition sketch for Turbulent oscillatory boundary layers at high Reynolds numbers (Jensen, Sumer, and Fredsoe 1989)

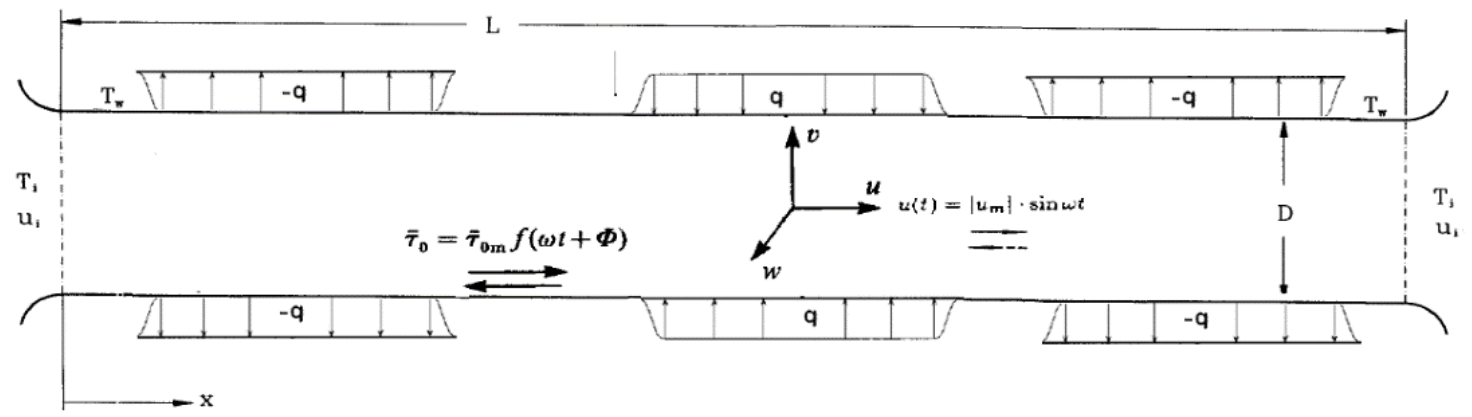

Figure 2. 9: The geometry and boundary conditions for the RMDHL study 
prove to be inappropriate and insufficient as they do not capture critical phenomena like phase lag in heat transfer and the influence of the oscillation frequency on the heat transfer (C Walther, Kühl, and Schulz 2000)(O. Popoola and Cao 2016).

Specific dissimilarities between oscillating flow and dynamically driven flow have been itemized, and the shortfalls in the correlations have been highlighted (Allan 2014). While some of these correlations provide a very close approximation, the shortfall in many other predictions is as much as $25 \%$. In some cases, inaccuracies in results were observed when correlations are wrongly copied by authors or when boundaries for application of the correlations were not correctly specified. Furthermore, the most recent literature review on correlations for oscillatory flow ( $\mathrm{T}$. S. Zhao and Cheng 1998b) was close to two decades ago.

Majority of the investigations on the heat transfer in turbulent oscillating pipe flow has focused on the Stirling engines, Vuilleumier heat pumps type systems, or high Reynolds number open channel applications. These systems typically use two-cylinder volumes, which are located at different temperature levels. The volumes are periodically changed by pistons and displacers, forcing the working fluid to oscillate within the process (Lee, Park, and Tack Ro 1998), for the heat removal from and heat addition to the working fluid in two separate analysis. Figures 2. 6 to 2.8 show the schematics for the majority of the oscillatory pipe flows reviewed in literature.

The goal of this section of the review is to provide an updated compilation of the heat transfer and flow characteristics for oscillating correlation that have been 
applied in literature. The review is necessary as a result of the challenges we experienced during the design process for the RMDHL.

\subsubsection{Dimensionless Numbers associated with Oscillatory Flow}

Oscillatory flow cooling is a combination of oscillatory flow and heat transfer. Substantial research has been undertaken in recent years for single-phase oscillatory flow heat transfer, mainly using air as the working fluid. Some of the most significant research efforts include the work of Cooper et al. (1994), Zhao and Cheng, (1995), Li and Yang (2000), and Sert and Beskok (2003). Cooper and Yang (1994) experimentally investigated convective heat transfer from a heated floor section of a rectangular duct to a low frequency, sizeable tidal displacement, oscillatory flow. The flow is substantially an oscillatory flow using air as the working fluid under the conditions of relatively sizeable oscillatory stroke and relatively low oscillatory frequency. They found that the oscillatory flow Nusselt number would depend on a single dimensionless parameter, $(l / d) \operatorname{Re}_{\omega}^{3 / 2}$, where $l$ is the heater length, $d$ is one-half of the duct height, and $\operatorname{Re}_{\omega}$ is the oscillating flow Reynolds number, which is defined as

$$
\operatorname{Re}_{\omega}=\frac{2 \pi \omega \Delta x 2 d}{v}
$$

where $\omega$ is the fluid oscillation frequency, $\Delta x$ is the fluid tidal displacement (one-half the flow cross-section averaged distance a fluid particle would move in a complete oscillation cycle). 
$\mathrm{Li}$ and Yang (2000) further performed a three-dimensional numerical simulation and compared their numerical results with the experimental data of Cooper and Yang (1994), with the purpose of determining the underlining mechanism for the observed heat transfer enhancement. They believed that the intra-cycle fluid oscillations promote fluid mixing and thus lead to the enhanced heat transfer. Zhao and Cheng (1995) presented a numerical solution for laminar forced convection of a reciprocating air flow in a pipe of finite length at a constant wall temperature. They believed that the heat transfer is governed by four dimensionless parameters: the Prandtl number, $\operatorname{Pr}$, the kinetic Reynolds number, $\mathrm{Re}_{\omega}$, the dimensionless oscillation amplitude of the fluid, $A_{o}$, and the length to diameter ratio of the heated tube, $L / D$. Unlike the oscillating flow Reynolds number defined by Cooper and Yang (1994), the kinetic Reynolds number here is based solely on the oscillation or reciprocating frequency, $\omega$, and is given by the following relation:

$$
\operatorname{Re}_{\omega}=\frac{\omega D^{2}}{v}
$$

The dimensionless oscillation amplitude of the fluid here is equivalent to the dimensionless fluid tidal displacement of Cooper and Yang (1994) and is defined as:

$$
A_{o}=x_{\max } / D
$$

where $x_{\max }$ is the maximum fluid displacement.

Unlike conventional unidirectional flows, mathematical description of the oscillatory flow is complicated and not easy to interpret. Particular integral and the general solutions to the homogeneous equation governing equations for oscillating flow 
is for the most part too complicated to provide explicit information (Chen, Luo, and Dai 2007)(Currie 2003). Depending on the physical description of the problem and the coordinate system adopted, the solution contains complex numbers, Bessel functions, and hyperbolic trig and exponential terms (Uchida 1956). In most cases, these terms require additional simplification. For instance Eq. (4) is the solution for the dimensionless temperature profile for single phase, oscillating, laminar, incompressible flow in a parallel wall channel, with a constant wall temperature (Grossman and Nachman 2006).

$\frac{\mathrm{T}-\mathrm{T}_{\mathrm{L}}}{\mathrm{T}_{\mathrm{H}}-\mathrm{T}_{L}}=\frac{\mathrm{L}-\mathrm{x}}{\mathrm{L}}+\left(\frac{\mathrm{a}^{4} \Delta \mathrm{P}}{\rho v^{2} \mathrm{~L}^{2}}\right) \frac{\sqrt{\mathrm{Pr}}}{4 \mathrm{~V}^{4}(\mathrm{Pr}-1)\left(\sinh ^{2} \mathrm{~V}+\cos ^{2} \mathrm{~V}\right)} \times$

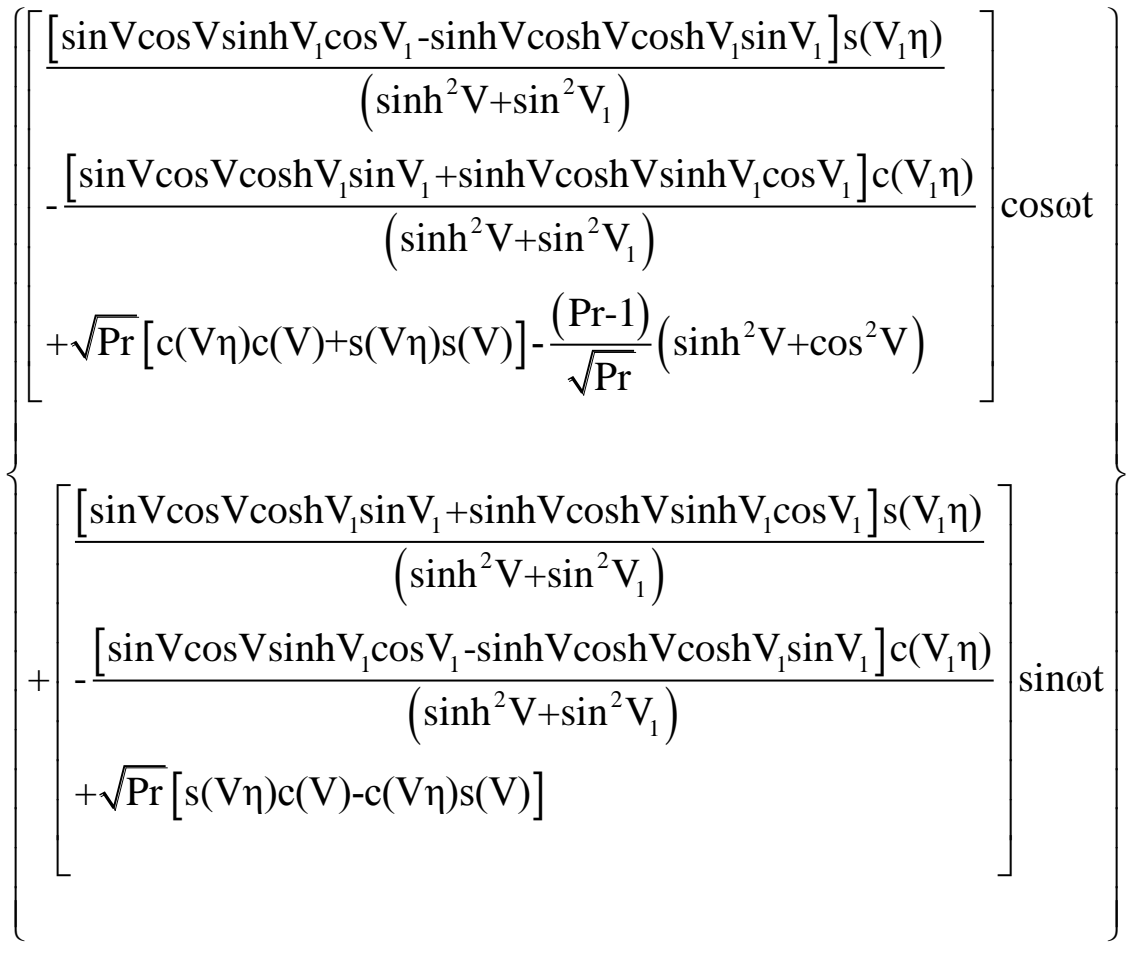


where $c(z)=\cosh (z) \cos (z) ; s(z)=\sinh (z) \sin (z) ; \eta=y / a$ is the dimensionless lateral coordinate, and $V=\sqrt{\frac{V a}{8}}$.

Due to the significant increase in the number of new thermal management devices that involve oscillating flow and the potential for proprietary applications involving oscillating flows, there have been significant research work in addressing the challenges associated with aspects of the fluid flow and heat transfer characteristics of the RMDHL and other such heat transfer devices which require an oscillating flow of the working fluid. Some of these works were to:

i. provide further insight into the mechanism for improved heat transfer in oscillating flow over conventional flow (P. Li and Yang 2000),

ii. implications of the so-called annular effect where the maximum axial velocity in some oscillatory flow occurs near the wall first observed by Richardson and Tyler (Richardson and Tyler 1929; Kuosa et al. 2012; Xiao, Zhou, et al. 2014).

More significant for inventors and designers for thermal management systems among these research is work improve the accuracy of the analytical correlations associated with the quantifying system fluid mechanics and thermal variables. Some of these correlations developed an approximate analytical and numerical solution of the Navier Stokes equation and experimental measurements. Majority of the research into the correlations span several decades, and several researchers from different countries and are yet to be harmonized. 


\subsubsection{Governing Equations}

A thermal management application that employs oscillatory flow of the working fluid within a channel, a pipe or a combination of channels and pipes where the fluid is driven by a sinusoidal displacer is equivalent to a problem of an incompressible, viscous fluid oscillating within a confined boundary connecting two reservoirs (hot reservoir at $\mathrm{T}_{\mathrm{H}}$ and a cold reservoir at $\mathrm{T}_{\mathrm{C}}$. Oscillating turbulent regimes has been described by (C Walther, Kühl, and Schulz 2000) to mean that there are phases in which the flow is turbulent while laminar flow occurs during acceleration after flow reversal.

The governing equations are the continuity equation, the Naiver-Stokes equation, and the Energy equations. However, for ease of analysis, a number of different formulations of the governing equations have been developed based on dimensionless coordinates and parameters. Given a sinusoidal flow is that is either prescribed by a sinusoidal mean velocity $\left(u_{m}\right)$ (Eq. (5)) with a maximum velocity $\left(u_{\max }\right.$ ) (Eq. (6)) or driven by sinusoidally varying pressure gradient ( $\partial p / \partial x)($ Eq. (7)) and possible phase angle $(\phi)$ (Eq. (8)), the most common formulations of the governing equations are in terms of oscillation angular frequency $(\omega)$ is the time $(t)$, pipe or channel (diameter (D) or width (h)), stream function $(\Psi)$, kinematic viscosity $(v)$ density $(\rho)$ and thermal conductivity $\left(k_{f}\right)$ is as follows:

$$
u_{m}=u_{\max } \sin \phi
$$




$$
\begin{aligned}
& u_{\max }=\frac{x_{\max } \omega}{2} \\
& -\frac{\partial p}{\partial x}=p_{o} \cos \phi \\
& \omega t=2 \pi(i-1)+\phi
\end{aligned}
$$

Continuity:

$$
\begin{gathered}
\nabla \cdot \vec{V}=0 \\
\nabla^{2} \Psi=-\zeta
\end{gathered}
$$

(T. S. Zhao and Cheng 1998b)

(Iliadis and Anagnostopoulos 1998)

\section{Momentum:}

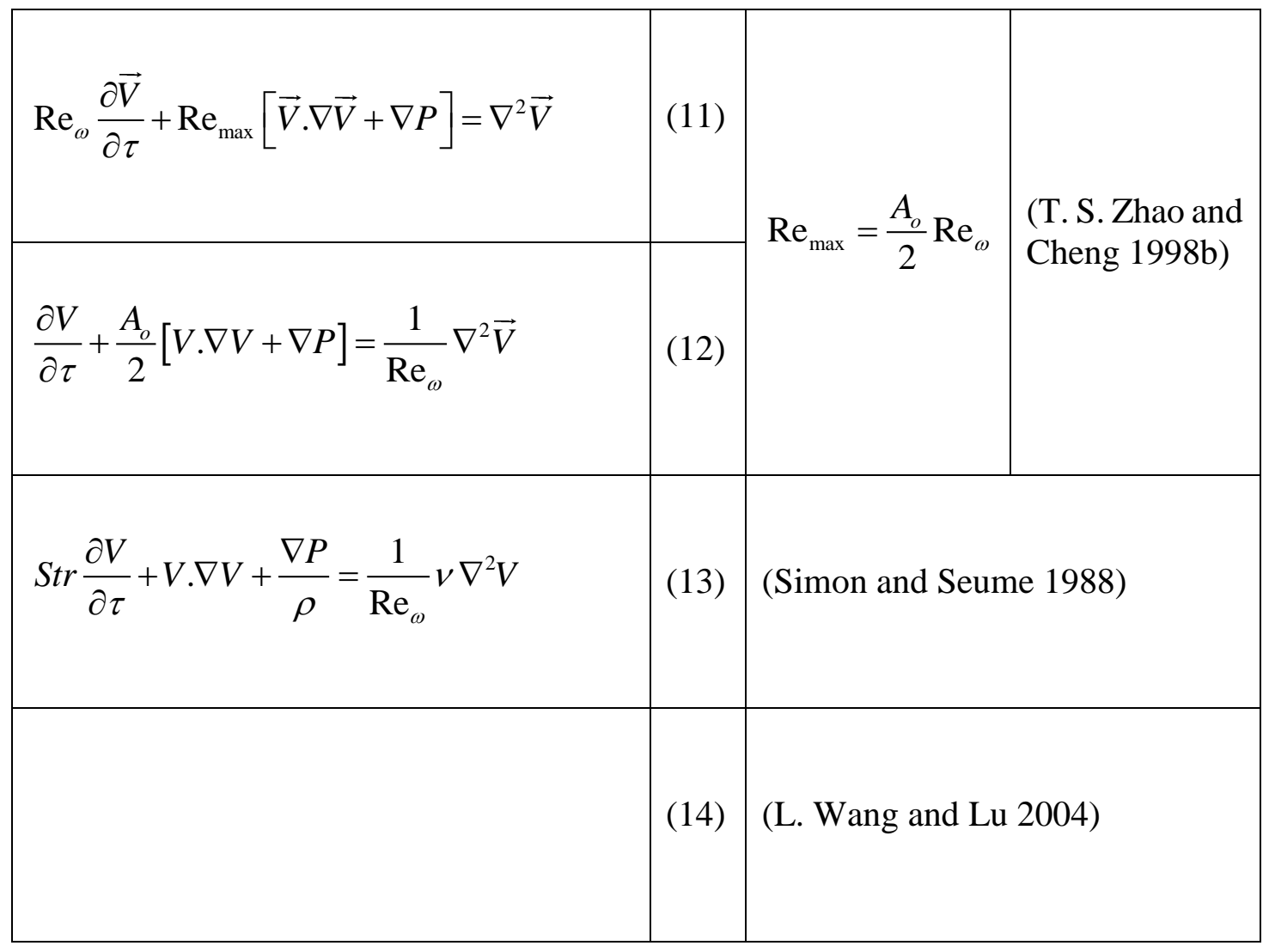




\begin{tabular}{|c|c|c|}
\hline $\begin{aligned} \frac{\partial \overline{u_{i}}}{\partial t}+\frac{\partial}{\partial x_{j}}\left(\overline{u_{i}} \bar{u}_{j}\right) & +\frac{\partial \bar{p}}{\partial x_{i}} \\
& =\frac{1}{\operatorname{Re}_{\tau}} \frac{\partial^{2} \bar{u}_{i}}{\partial x_{j} \partial x_{j}}-\frac{\partial \tau_{i j}}{\partial x_{j}}+P_{g} \delta_{i 1}\end{aligned}$ & & \\
\hline$\frac{\partial \zeta}{\partial t}+\frac{\partial \Psi}{\partial y} \frac{\partial \zeta}{\partial x}-\frac{\partial \Psi}{\partial x} \frac{\partial \zeta}{\partial y}=v \nabla^{2} \zeta$ & (15) & $\begin{array}{l}\text { (Iliadis and Anagnostopoulos } \\
\text { 1998) }\end{array}$ \\
\hline $\begin{aligned} \frac{\partial \zeta}{\partial t}+\frac{1}{r}\left(\frac{\partial}{\partial r}\left(\zeta \frac{\partial \Psi}{\partial \theta}\right)\right) & \\
-\frac{\partial}{\partial \theta}\left(\zeta \frac{\partial \Psi}{\partial r}\right) & =v \nabla^{2} \zeta\end{aligned}$ & (16) & $\begin{array}{l}\text { (Zhang, Dalton, and Brown 1993; } \\
\text { Justesen 1991) }\end{array}$ \\
\hline $\begin{aligned} g(\xi) \frac{\partial \zeta}{\partial t}+\left(\frac{\partial}{\partial \xi}\right. & \left.\left(\zeta \frac{\partial \Psi}{\partial \eta}\right)\right) \\
& -\frac{\partial}{\partial \eta}\left(\zeta \frac{\partial \Psi}{\partial \xi}\right)=\frac{2}{\operatorname{Re}} \nabla^{2} \zeta\end{aligned}$ & (17) & \\
\hline
\end{tabular}

Energy

\begin{tabular}{|l|l|l|}
\hline$\frac{\partial \bar{T}}{\partial t}+\frac{\partial}{\partial x_{j}}\left(\bar{T} \overline{u_{j}}\right)=\frac{1}{\operatorname{Re}_{\tau} \operatorname{Pr}} \frac{\partial^{2} \bar{T}}{\partial x_{j} \partial x_{j}}-\frac{\partial q_{j}}{\partial x_{j}}$ & (18) & (L. Wang and Lu \\
\hline
\end{tabular}




\begin{tabular}{|c|c|c|c|}
\hline $\begin{aligned} \rho c_{p}\left[2 \operatorname{Re}_{\omega} \frac{\partial T}{\partial t}\right. & \left.+\operatorname{Re}_{\max } u . \nabla T\right] \\
& =2 E c \operatorname{Re}_{\omega} \frac{\partial^{2} P}{\partial t} \\
& -E c \operatorname{Re}_{\omega} u . \nabla P \\
& +\frac{\nabla \cdot(k \nabla T)}{\operatorname{Pr}} \\
& +E c \phi\end{aligned}$ & (19) & $E c=\frac{u_{\max }^{2}}{c_{p}\left(T_{H}-T_{L}\right)}$ & $\begin{array}{l}\text { (Simon and } \\
\text { Seume 1988) }\end{array}$ \\
\hline$\frac{\partial \theta}{\partial \tau}+\frac{A_{o}}{2}(\vec{V} . \nabla) \theta=\frac{1}{\operatorname{Re}_{\omega} \operatorname{Pr}} \nabla^{2} \theta$ & $(20)$ & $\begin{array}{l}\theta=\frac{T-T_{C}}{T_{H}-T_{C}} \\
\theta=\frac{k_{f} T}{q_{w} D}\end{array}$ & $\begin{array}{l}\text { (T. S. Zhao and } \\
\text { Cheng 1998b) }\end{array}$ \\
\hline
\end{tabular}

The various dimensionless parameters in Eq. (9) to Eq. (20) are defined as $\operatorname{Re}_{\omega}=\omega D^{2} / v ;$ of the fluid; $\operatorname{Re}_{\text {max }}=u_{\max } D^{2} / v ; \operatorname{Str}=\omega d / u_{\max } ;(X, R)=(x / D, r / D)$ $; \tau=\omega t ; U=u / u_{\max } ; P=p / \rho u_{\max }^{2}$. Equations (9), (11) and (19) in terms of the $\operatorname{Re}_{\max }$ and $\mathrm{Re}_{\omega}$ are the most common form of the non-dimensional forms of the governing equations. However it has been shown that $\operatorname{Re}_{\text {max }}$ and $\operatorname{Re}_{\omega}$ play somewhat similar roles (T. S. Zhao and Cheng 1998a) and the fact that amplitude and frequency of oscillation are independent of each other, the form of the Governing Equation presented in 9 and 17 in terms of $A_{o}$ and $\mathrm{Re}_{\omega}$ had been adopted by other literature. In the studies where channel enhancements like baffles, rectangular and cylindrical cross-channel block and gutters are adopted into enhancing heat transfer in the oscillating flow, the vorticity formulation in (10), (15) and (16) are most adopted by researchers. For (10), 
(15) and (16), $u=(1 \backslash r)(\partial \Psi \backslash \partial \theta)$ and $v=-\partial \Psi \backslash \partial r$. This formulation removes pressure as an independent variable and allows coordinate transformations that can concentrate mesh spacing, and large mesh gradients. Eq. (17) is a non-dimensional form of Eq. (16) transformed using $\xi$ and $\eta$ as transformation variables. Eq. (19) provides for characterizing heat dissipation using the Eckert number $(E c)$. Additional details on how the governing equations are reduced to the various forms are provided in (T. S. Zhao and Cheng 1998b; Simon and Seume 1988; L. Wang and Lu 2004; Iliadis and Anagnostopoulos 1998; Zhang, Dalton, and Brown 1993).

\subsubsection{Velocity Profile}

The structure of for internal oscillating pipe flow is involved, several aspects of the flow structure are yet to be fully understood. Over time, Fourier, Laplace, Green and a number of other mathematical methods have been adopted for solving the governing equations. The chronologic development and the State of the art for an analytical solution to the oscillating internal flow have been presented in (Blythman et al. 2016). The earliest direct solution for the velocity profile for oscillatory flow pipe was provided by Richard and Tyler (1929) (Richardson and Tyler 1929) and is given as follows:

$u(r)=\frac{p_{o}}{i \omega \rho}\left[1-\frac{J_{o}(\lambda r)}{J_{o}(\lambda R)}\right]$

$$
\lambda=\frac{i \omega \rho}{\mu}
$$




\begin{tabular}{|l|l|}
\hline$Q_{o}=\frac{\pi R^{2} p_{o}}{i \omega \rho}\left[1-\frac{2 J_{o}(\lambda R)}{\lambda R J_{o}(\lambda R)}\right]$ & $(22)$ \\
\hline$J_{o}$ denotes the Bessel function of the first kind and \\
$\begin{array}{l}\text { is of zero order } J_{o}(\lambda r)=\operatorname{ber}(\lambda r)+\operatorname{bei}(\lambda r) \\
\text { (Abramowitz and Irene A. } \\
\text { Stegun 1972) }\end{array}$ \\
\hline
\end{tabular}

For real application, (21) and (22) needs to be decomposed to yield the real part explicitly. Significant effort has been undertaken to visualize the flow and thereby verify the solution using various experiments (Harris, Peev, and Wilkinson 1969; Simon and Seume 1988; Ch. Walther et al. 1998). In (Simon and Seume 1988) plots of velocity profiles for laminar, incompressible, fully developed, oscillating flow for eight dimensionless frequencies the plots are provided. It is evident from the figure that this flow is characterized by a distinct volatility and acute dependence on the velocity of oscillating flow. This can be attributed to viscous dissipation that occurs. As a result of the change if flow direction, the oscillating flow transitions from different degrees of turbulence and laminar flow in a single cycle(Iguchi, M., Ohmi, M., \& Maegawa 1982). Fig 2. 10 shows the variation of velocity profiles in laminar oscillating pipe flow with time during one cycle (Ch. Walther et al. 1998).

Alternate solutions for the velocity profile of oscillatory flow of fluid in a pipe driven by a sinusoidal pressure gradient was integrated over the cross-section of a pipe as given by Eq. (5) and simplified for the Reynolds number range covering the value obtained in Eq. (9) (T. S. Zhao and Cheng 1998b) and (Uchida 1964): 


$$
\begin{aligned}
u & =\left(\frac{k D^{2}}{4 \alpha^{2} v}[B \cos \omega t+(1-A) \sin \omega t]\right) \\
u_{\max } & =\frac{k D^{2} \sigma}{32 v}
\end{aligned}
$$

where:

$$
\begin{aligned}
& A=\frac{\operatorname{ber}(\alpha) b e i(2 \alpha R)-\operatorname{bei}(\alpha) \operatorname{ber}(2 \alpha R)}{\operatorname{ber}^{2}(\alpha)+\operatorname{bei}^{2}(\alpha)} \\
& B=\frac{\operatorname{ber}(\alpha) \operatorname{ber}(2 \alpha R)-\operatorname{bei}(\alpha) \operatorname{bei}(2 \alpha R)}{\operatorname{ber}^{2}(\alpha)+\operatorname{bei}^{2}(\alpha)} \\
& \phi=\frac{\pi}{2}(\omega t-\Lambda) \\
& \Lambda=\tan ^{-1}\left(\frac{\alpha-2 C_{1}}{2 C_{2}}\right) \\
& E=\left[1-\left(\frac{r}{R}\right)^{2}\right] \sqrt{\frac{R e_{\omega}}{8}} \\
& \sigma=\frac{8}{\alpha^{3}} \sqrt{\left(\alpha-2 C_{1}\right)^{2}+4 C_{2}^{2}} \\
& C_{1}=\frac{\operatorname{ber}(\alpha) \operatorname{bei}^{\prime}(\alpha)-\operatorname{bei}^{2}(\alpha) \operatorname{ber}^{\prime}(\alpha)}{\operatorname{ber}^{2}(\alpha)+\operatorname{bei}^{2}(\alpha)} \\
& C_{2}=\frac{\operatorname{ber}(\alpha) \operatorname{bei}^{\prime}(\alpha)+\operatorname{bei}^{2}(\alpha) \operatorname{ber}^{\prime}(\alpha)}{\operatorname{ber}^{2}(\alpha)+\operatorname{bei}^{2}(\alpha)}
\end{aligned}
$$

ber( ), bei( ), are kelvin functions(Richardson and Tyler 1929; Abramowitz and Irene A. Stegun 1972) defined as:
$\operatorname{ber}^{\prime}(x)=\frac{d(\operatorname{ber}(x))}{d(x)}$
and

$$
\operatorname{bei}^{\prime}(x)=\frac{d\left(b e i^{\prime}(x)\right)}{d(x)}
$$




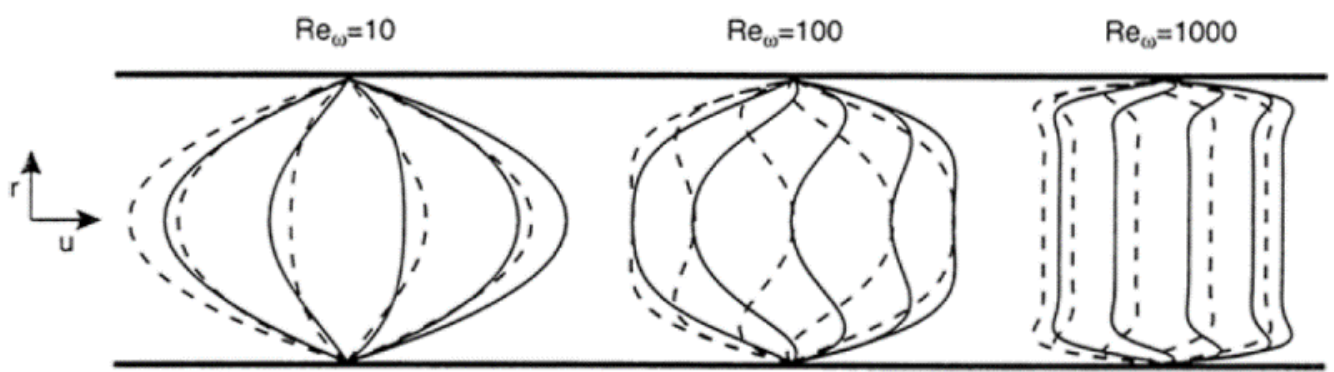

Figure 2. 10 Variation of velocity profiles in laminar oscillating pipe flow with time during one cycle. Fluid Accelerated to the right (Ch. Walther et al. 1998) 
For reciprocating flow in the rectangular, the analytical solution for the flow between two parallel planes when there is pressure gradient which varies harmonically with the time given by Landau and Lifshitz (Landau and Lifshitz 1987) provided in Eq. (19) to Eq. (25) was used in this study.

$$
\frac{\partial V}{\partial t}=a e^{i \omega t}+v \frac{\partial^{2} V}{\partial y^{2}}
$$

Subject to boundary conditions $v=0$ for $\mathrm{y}= \pm \mathrm{h}$. If the $x z$ plane is half-way between the two planes, the solution can be rewritten in terms of the pump parameters as:

$$
\begin{aligned}
& V=\frac{i a}{\omega} e^{i \omega t}\left(1-\frac{\cos k y}{\cos \frac{k H}{2}}\right) \\
& k=\frac{\alpha(i+1)}{H \sqrt{2}} \\
& i \omega=v k^{2}
\end{aligned}
$$

Eq. (23) to Eq. (36) are given in terms of the Womersley number $(\alpha)$ defined in Eq. (37), the importance of $\alpha$ is in its relationship to the thickness of the 'Stokes layer' ( $\delta$ ) defined in Eq. (38). $\delta$ and $\alpha$ play a significant role in defining the velocity profile 
due to their relationship to $\omega$. This is because the $\omega$ is a critical determinant of the generation and dissipation of the viscous forces in the flow.

$$
\begin{aligned}
& \alpha=\frac{\mathrm{D}}{2} \sqrt{\frac{\omega}{v}} \\
& \delta=\sqrt{\frac{2 v}{\omega}}=\frac{D \sqrt{2}}{\alpha}
\end{aligned}
$$

Additional explicit decomposition of the real portion of the velocity profile is presented in Eq. (39) to Eq. (47). Eq. (39) and Eq. (43) are the velocity profile for a two-dimensional oscillatory flow in a semi-infinite plane above a rigid wall as presented by (Ramos et al. 2004). The solution is based on the assumption that the velocity has no variation in the perpendicular direction to the wall. For oscillating pressure driven flow, Eq. (40) is the mean flow over a cross-section of a channel based on Eq. (34). Eq. (41) and Eq. (42) are limiting cases of Eq. (40) with simplifications based on the width of the channel. Similarly, Eq. (44) and Eq. (45) are limiting cases of Eq. (23) with simplifications based on the oscillating frequency of the flow. Eq. (47) is the average velocity for turbulent oscillating pipe flow. Eq. (46) is a solution for the pressure driven oscillating pipe flow between two parallel plates derived using the Green theorem decomposition. A three-dimensional solution of the governing equation of oscillating flow in the $\mathrm{r}-\theta$-x coordinate is presented in (Xiao, Zhou, et al. 2014). The solution was obtained using Maple soft. 


\begin{tabular}{|c|c|c|c|}
\hline$u(y, t)=U_{\infty}\left[\cos (\omega t)-\left(\cos (\omega t)-\left(\frac{\omega}{2 v}\right)^{112} y\right) \exp ^{\left[-\left(\frac{\omega}{2 v}\right)^{12} y\right.}\right]$ & (39) & oscillatory boundary layer flow & (Ramos et al. 2004) \\
\hline$\overline{u(y, t)}=\frac{i P_{x}}{\omega} e^{-i \omega t}\left(1-\left(\frac{2}{\frac{h(1+i)}{\delta}} \tan \left[\frac{1}{2} \frac{h(1+i)}{\delta}\right]\right)\right)$ & (40) & & \multirow{3}{*}{$\begin{array}{l}\text { (Landau and Lifshitz } \\
\text { 1987) }\end{array}$} \\
\hline$\overline{u(y, t)}=P_{x} e^{-i \omega t}\left(\frac{h^{2}}{12 v}\right)$ & (41) & $\frac{h}{\delta}>1$ & \\
\hline$\overline{u(y, t)}=\frac{i P_{x}}{\omega} e^{-i \omega t}$ & (42) & $\frac{h}{\delta}>1$ & \\
\hline$U(Y, \tau)=e^{-\eta} \cos (\tau-\eta)$ & (43) & $\begin{array}{l}\eta=\frac{y}{\delta} \\
U(Y, \tau)=\frac{u(y, t)}{u_{\max }} \\
\text { Flat plate with infinite length }\end{array}$ & \multirow{3}{*}{$\begin{array}{l}\text { (T. S. Zhao and Cheng } \\
\text { 1998b) }\end{array}$} \\
\hline $\begin{array}{l}U(r, \tau)=\frac{2 \cos \tau}{a \sigma}\left[1-\left(\frac{r}{a}\right)^{2}\right]+ \\
\quad \frac{1}{32 a \sigma} \operatorname{Re}_{\omega}\left[\left(\frac{r}{a}\right)^{4}+4\left(\frac{r}{a}\right)^{2}-5\right] \sin \tau\end{array}$ & (44) & $\begin{array}{l}\operatorname{Re}_{\omega}<16 \\
U(r, \tau)=\frac{u(r, t)}{u_{\max }}\end{array}$ & \\
\hline$U(r, \tau)=\frac{32}{a \sigma \operatorname{Re}_{\omega}}\left[\sin \tau-\frac{e^{-E}}{\sqrt{r / a}} \sin (\tau-E)\right]$ & (45) & $\operatorname{Re}_{\omega}>16 ; U(r, \tau)=\frac{u(r, t)}{u_{\max }}$ & \\
\hline
\end{tabular}




\begin{tabular}{|c|c|c|c|}
\hline $\begin{aligned} U^{\prime}(y, z, t)= & \left(-\frac{16 \nabla P}{\rho \pi^{2}} \sum_{m=0}^{\infty} \sum_{n=0}^{\infty} \frac{Z}{(2 m+1)(2 n+1)}\right) . \\
& \left(\frac{v \beta \cos (\omega t)+\omega \sin (\omega t)-v \beta e^{\nu \beta t}}{v^{2} \beta^{2}+\omega^{2}}\right)\end{aligned}$ & $(46)$ & $\begin{array}{l}Z=\sin \left(\frac{(2 m+1) \pi y}{a}\right) \sin \left(\frac{(2 n+1) \pi z}{b}\right) \\
\beta=\left(\frac{(2 m+1) \pi}{a}\right)^{2}+\left(\frac{(2 n+1) \pi}{b}\right)^{2}\end{array}$ & (Blythman et al. 2016) \\
\hline$|\bar{u}|=\frac{1}{\kappa}\left(\frac{\operatorname{Re}_{R}}{\operatorname{Re}_{A}}\right)^{0.25}\left[0.25 \ln \left(\operatorname{Re}_{A}\right)+0.75 \ln \left(\operatorname{Re}_{R}\right)+1.92\right]$ & $(47)$ & $\begin{array}{l}0.38 \leq \kappa \leq .47 \\
\kappa=\text { von Kármán constant } \\
\operatorname{Re}_{A}=\frac{2 \pi f A^{2}}{v} \\
\operatorname{Re}_{R}=\frac{2 \pi f R^{2}}{v}\end{array}$ & $\begin{array}{l}\text { (Su, Davidson, and } \\
\text { Kulacki 2012) }\end{array}$ \\
\hline
\end{tabular}




\subsubsection{Transition to turbulence}

The answer the question of where the transition from laminar to turbulent flow occurs in oscillating flow is a complex one as there is a different standard for flow description. These variations take 3 distinct forms. First, when one looks at the classification flow description for oscillating flow, some describe the flow in terms of Reynolds number (Re), Reynolds number angular velocity $\left(\operatorname{Re}_{\omega}\right)$, Stokes number, dimensionless oscillation amplitude of fluid $\left(\mathrm{A}_{\mathrm{o}}\right)$, Womersley number $(\alpha)$, depth of penetration $(\delta)$, Strouhal number $(\mathrm{St})$ and Valensi number $(\mathrm{Va})$ or at times critical number which is a combination of any of the listed terms. Secondly, there is a significant lack of agreement in the definition of the entrance length, limits for laminar, turbulent flow and definition of the transition flow(Simon and Seume 1988). Thirdly, oscillating flow is similar but fundamentally different from pulsating flow. However, they are used interchangeably in the literature.

The most popular nomenclature for the describing the transition to turbulence

in oscillating flow is the critical value $\beta_{c r i}=A_{o} \sqrt{\operatorname{Re}_{\omega}}$. The mechanism of transition to turbulence and the interplay of the transition has been presented by a number of Authors. Detailed Experimental investigation, as well as a table of the different transition points recorded by the various investigations, is provide in (T. S. Zhao and Cheng 1998b; Akhavan, Kamm, and Shapiro 1991; Ohmi and Iguci 1982; T. S. Zhao and Cheng 1996a) and more recently (Xiao, Zhou, et al. 2014). Eq. (48) and Eq. (49) are two widely adopted conditions for transition to turbulence. 


$$
\begin{aligned}
& \operatorname{Re}_{c}=305\left[\frac{D}{\delta_{v}}\right]^{1 / 7} \\
& A_{o} \sqrt{\operatorname{Re}_{\omega}}>761
\end{aligned}
$$

(49) (T. S. Zhao and Cheng 1996a)

\subsubsection{Turbulence Modelling for A Reciprocating Mechanism Driven Heat loop}

The types of flows that are typical of the RMDHL environment are characterized by unsteady (oscillatory flow and oscillatory pressure), 2-D/3-D (complex geometry), compressible (low Mach number), turbulent, and unsteady fluid flow as well as conduction and turbulent convection heat transfer. Other conditions include sudden changes in cross-section and isothermal and adiabatic boundary conditions (M. B. Ibrahim, Zhang, and Wei 2002)(M. B. Ibrahim, Zhang, and Kembhavi 2002). As a result, understanding the heat transfer and pressure drop in the RMDHL under operating conditions will require accurate and robust Computational Fluid dynamics (CFD) and turbulence modeling capability is critical to the success of the CFD. It is understandable that turbulence is arguably the most challenging area in CFD.

No single model or modeling approach can cover all types of turbulent flows; different types of turbulence models have been developed in the past decades (Nallasamy 1987; Markatos 1986; Gundogdu and Carpinlioglu 1999; Gündoğdu and Çarpınlıoğlu 1999; Özdinç Çarpinlioğlu and Yaşar Gündoğdu 2001; Ahn and Ibrahim

1991; M. Ibrahim and Hashim 1994; Su, Davidson, and Kulacki 2012; Versteeg and Malalasekera 2007; Ghafarian, Mohebbi-Kalhori, and Sadegi 2013; L. Wang and Lu 
2004; T. Zhao and Cheng 1995; Sert and Beskok 2003; Jensen, Sumer, and Fredsoe 1989). Due to the unavailability of suitable correlations for oscillating flow conditions in the literature, correlations derived from steady state unidirectional flow assumptions are generally adopted for reciprocating flows(C Walther, Kühl, and Schulz 2000; Lee, Park, and Tack Ro 1998; T. S. Zhao and Cheng 1998b; T. S. Zhao and P.Cheng 1996).

Ahn and Ibrahim (Ahn and Ibrahim 1991) conducted a two-dimensional oscillating flow analysis simulating the gas flow inside Stirling heat exchangers for laminar and turbulent oscillating pipe flow. In the study, the performance evaluation of the K- $\varepsilon$ model was made to explore its suitability to quasi-steady turbulent models in unsteady oscillating flow analysis. Ibrahim et al. (M. Ibrahim and Hashim 1994) investigated the usefulness of a low Reynolds number version of $k-\varepsilon$ to model the laminar, transitional, and turbulent flow in an oscillatory pipe flow between two parallel plates with a sudden change in cross section using. Su et al. (Su, Davidson, and Kulacki 2012) used the RANS method with Saffman's turbulence model to solve the time-dependent turbulent Navier-Stokes and energy equations for oscillating pipe flows.

Wang and $\mathrm{Lu}$ (L. Wang and Lu 2004) investigated oscillating turbulent channel flow with heat transfer using the large eddy simulation (LES) technique. The conclusion in all the works reviewed above is that the unidirectional flows models in some cases either do not include the effect of phase lag in heat transfer or does not reflect the influence of the oscillation frequency on the heat transfer and has sometimes 
proved to be inappropriate. Hence proper attention is required in the selection of a suitable model.

Turbulence models can be classified as zero, one, two or three equation models based on the number of partial differential equations solved in addition to the mean flow equation (Nallasamy 1987). The $k-\varepsilon, k-\quad$ and the $k-\omega$ models belong to the twoequation models. The standard and modified forms of the $k$ - $\varepsilon$ model is used in a majority of flows reported in the literature. While the equally popular Transition $k$ $-\omega$ and Transition SST are three equation models. This study will compare the accuracy of the predictions of the Standard k- $\varepsilon$ Model, Renormalization-group (RNG) k- $\varepsilon$ Model, Realizable k- $\varepsilon$ Model, Standard k- $\omega$, Shear-Stress Transport (SST) k- $\omega$ Model and Transition SST Model.

\subsubsection{Mass of vaporization $\left(m_{v}\right)$}

For an oscillating flow typical of the RMDHL power is required to move the working fluid around the loop, overcome friction, and in the case of phase change, power is required to overcome the inertia and body forces that arise. As a result instantaneous expansion during evaporation at the hotplate and gradual contraction at

the condenser. ${ }^{m}$ is required adequately quantify the power consumption during phase change. The exact characteristics of the thermodynamic cycle of the RMDHL are still unknown. this is because the rate of vapor generation and condensation at the evaporator and condenser is not constant. Twice within the cycle pressure and velocity 
become zero. At these points, the simultaneous heating and pressure increase of the fluid in the evaporator becomes a somewhat complicated process. These point in the process is characterized by a rate of phase change more rapid that the phase change at any other point in the cycle. These points will be accompanied by isentropic pumping of the working fluid due to phase change as well as superheating of the saturated vapor generated. Even though the exact nature of the mass vaporization and condensation have not been measured, These points have been captured (O. Popoola, Bamgbade, and Cao 2016) and significant efforts have been made to quantify $m_{v}$ (West 1982) as shown in Eq.(50) and Eq. (51). Fig 2. 12 show the expected pattern of the relative phasing of cylinder volume, mass of vapor, and evaporation condensation rate (West 1982) in an oscillating flow:

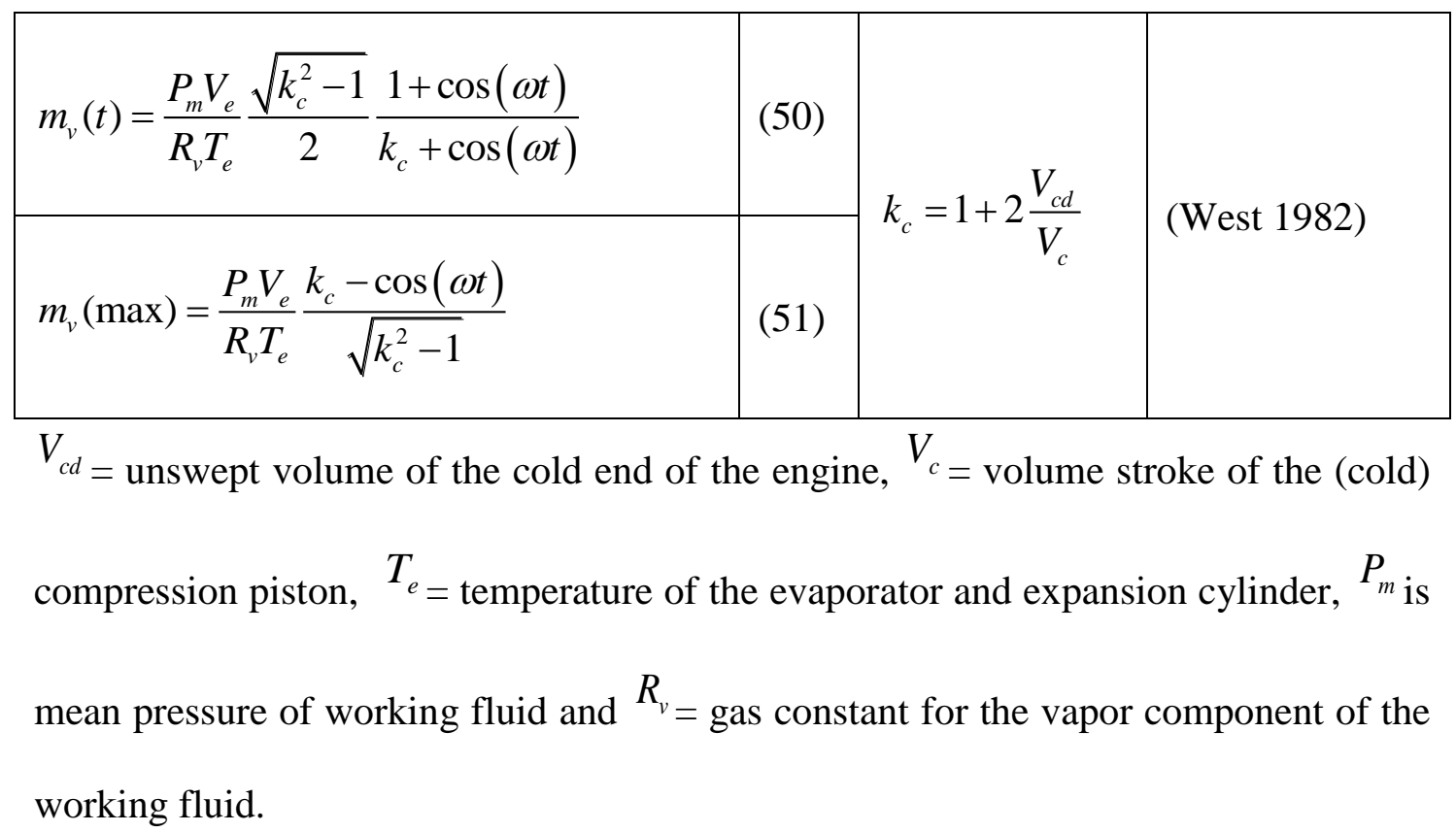




\subsubsection{Pressure drop}

Typical of regenerative heat transfer systems, understanding the pressure drop within the system is required to predict the thermodynamic equilibrium accurately, uniformity of temperature through the loop and phase change. Some of the analytical correlation for the prediction of the pressure drop in a reciprocating loop is given in Eq. (52) to Eq. (55). Eq. (52) present the differential equation for approximating the non-dimensional pressure drop ${ } / \rho u_{\max }^{2}$ for the developed laminar oscillating pipe. In addition to Eq. (52), (C Walther, Kühl, and Schulz 2000) also solved for the pressure drop in a turbulent oscillating flow using a phase averaging technique. Fig 2. 11: compares the solution of Eq. (52) with this "phase-averaging" method for values of $\mathrm{Re}_{\max }=30000$ and $R \omega=800$. Eq.(53) is a solution for the instantaneous value of the pressure drop per length in the axial direction of the flow. (Schwendig 1992), the source of Eq.(53) was not presented in English, the equation was presented as reported by (Barreno et al. 2015). (Barreno et al. 2015) derived Eq. (54) for laminar reciprocating flow $25 \leq \operatorname{Re}_{\omega} \leq 600 ; 1 \leq A_{o} \leq 600 ; 750 \leq \beta \leq 350$ and Eq. (55) for turbulent reciprocating flow $25 \leq \operatorname{Re}_{\omega} \leq 600 ; 1 \leq A_{o} \leq 600 ; 750 \leq \beta \leq 3000$. For certain combination of flow parameter, some research work has been able to separate the frictional loss component of the pressure drop. Cycle averaged Friction Coefficient is presented in Eq. (56) to Eq. (61) while the Cycle averaged friction factor is presented in Eq. (62) and Eq. (63). 


\subsubsection{Thermodynamic Principles}

The description of the thermodynamics of oscillating flow is closely approximated to the thermodynamic cycle of Stirling Engine. This is based on the assumption that the entire system can be modeled as a cylinder with pistons at both ends, the hightemperature sink located at the center and the cold heat sink distributed at both ends (Tziranis 1992). And as with all heat engines, it will have 4 processes heating accompanied by expansion and cooling associated with compression. The idealized stages are presented in Fig 2. 13.

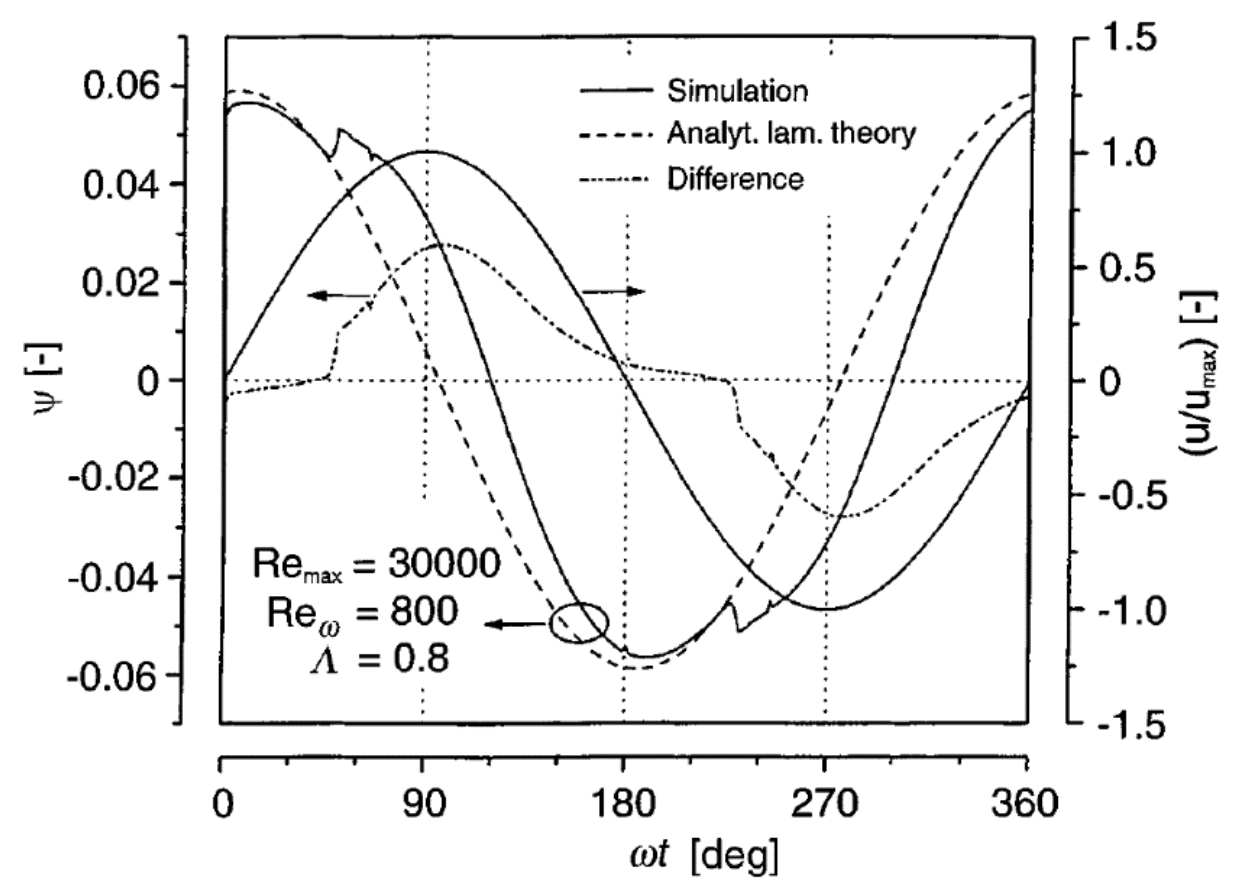

Figure 2. 11: Pressure drop in turbulent oscillating pipe flow compared to laminar theory (C Walther, Kühl, and Schulz 2000) 

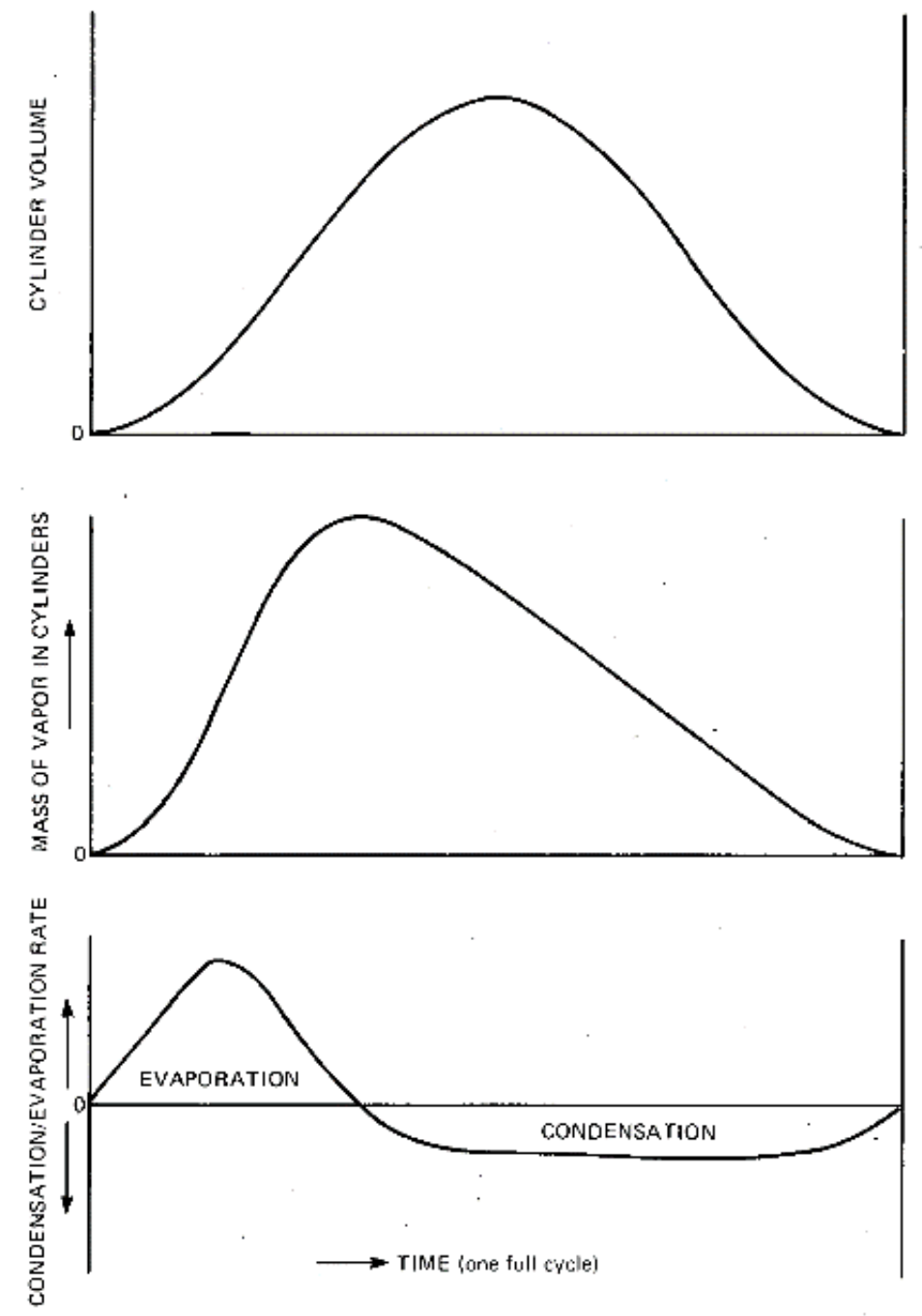

Figure 2. 12: Relative phasing of cylinder volume, mass of vapor, and evaporation condensation rate(West 1982) 


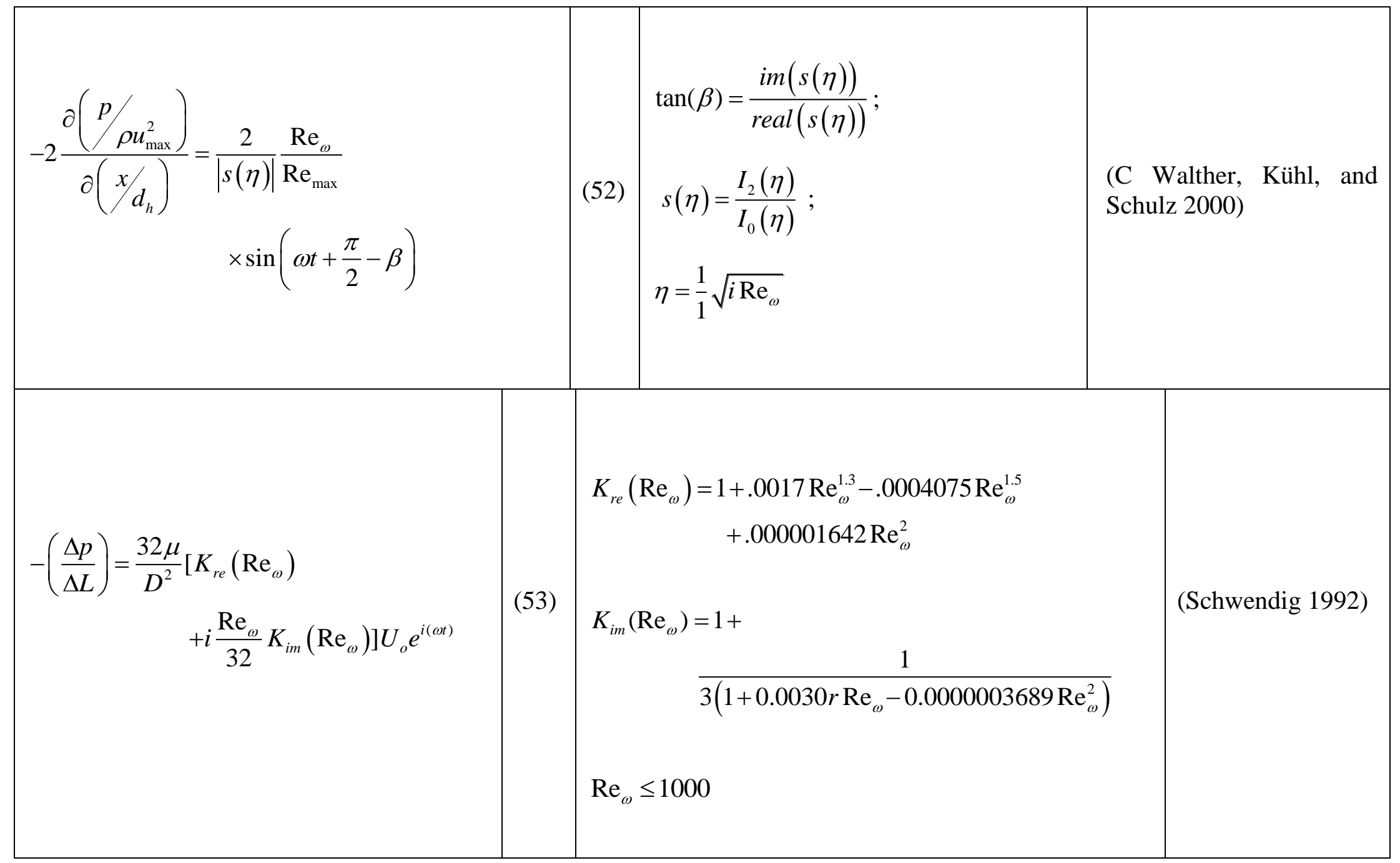




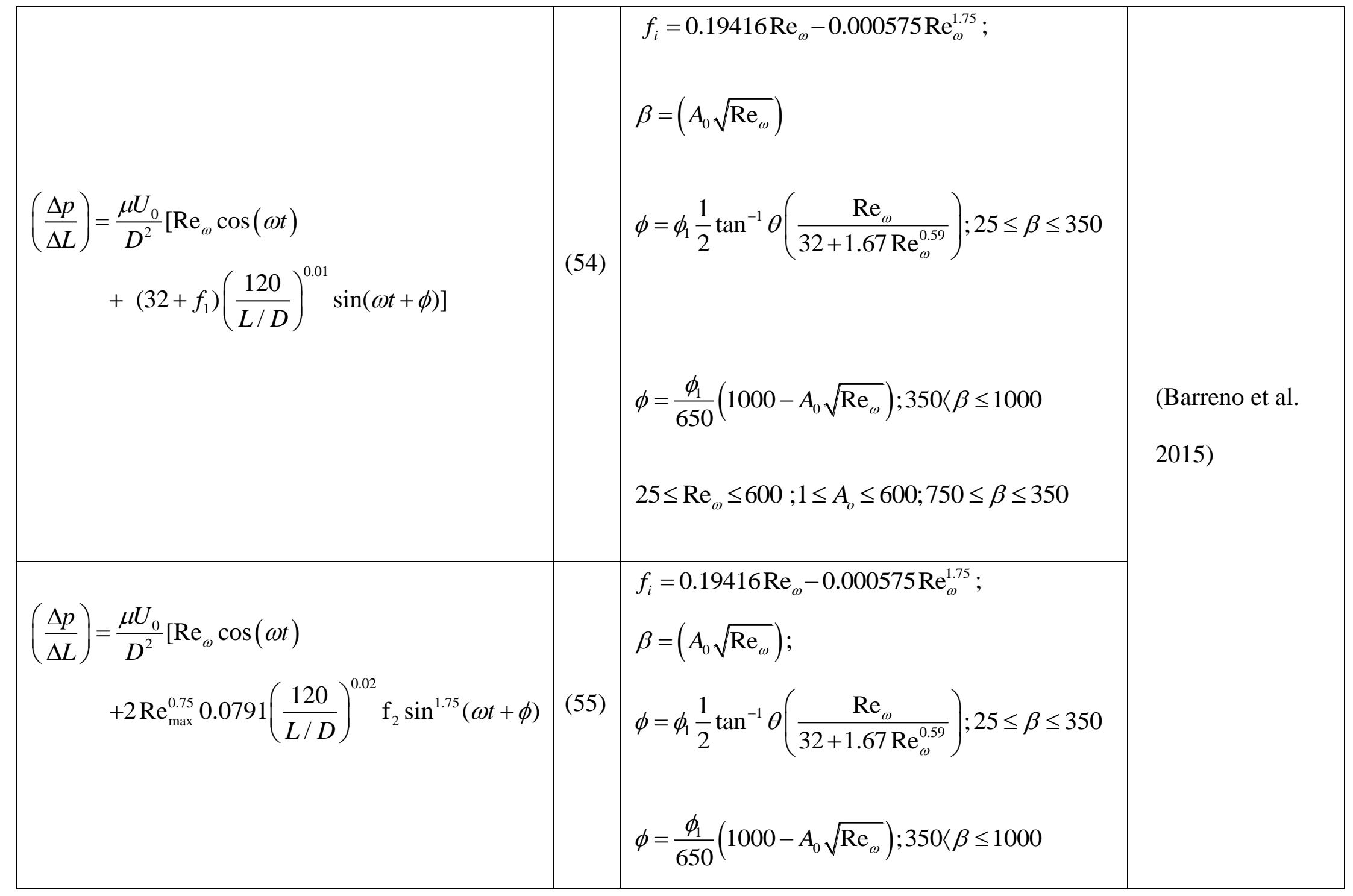




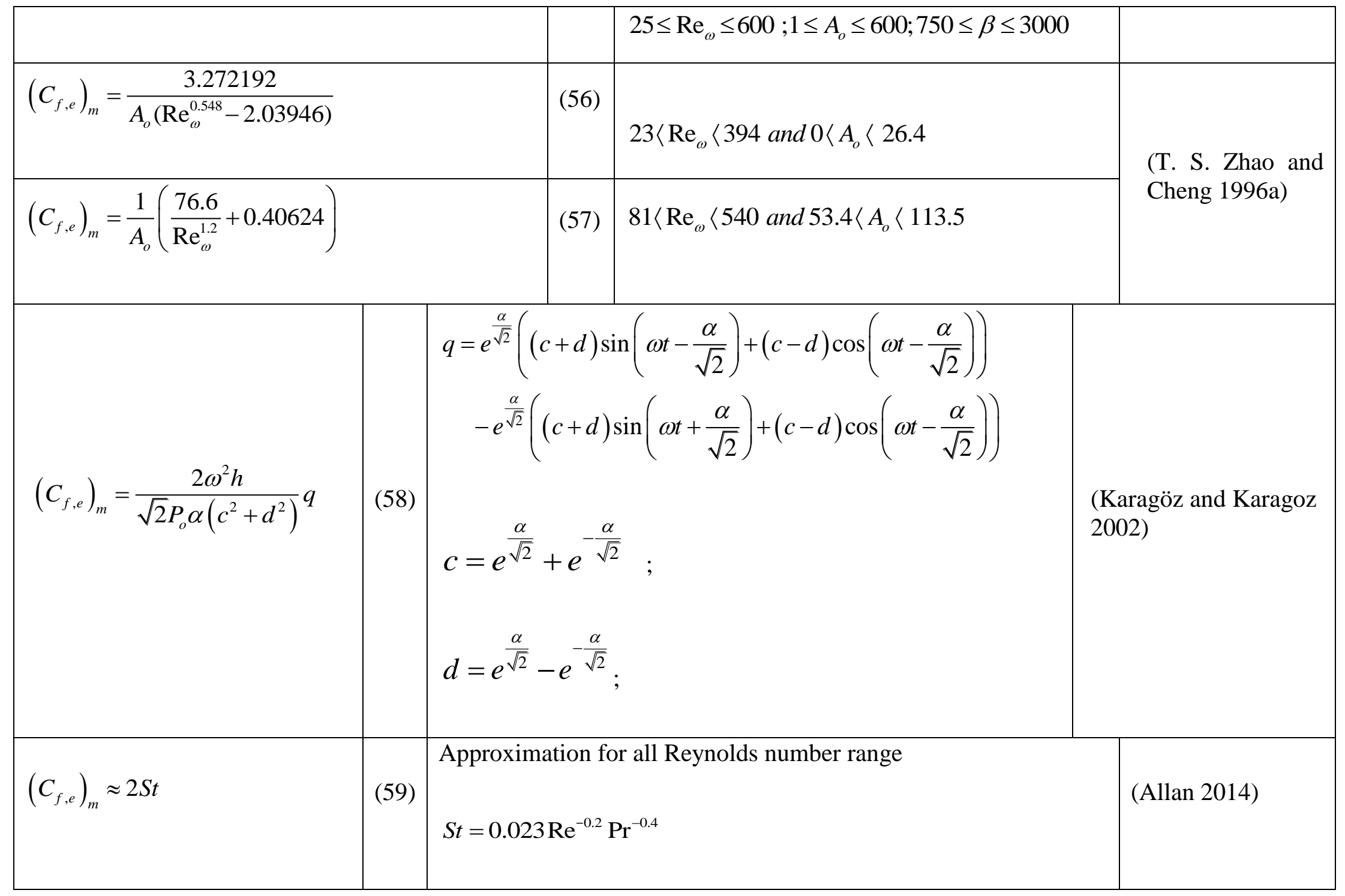




\begin{tabular}{|l|l|l|l|}
\hline$\left(C_{f, e}\right)_{m}=\frac{4}{\sqrt{\left(\operatorname{Re}_{\omega}\right)_{x}}} \sin \left(\omega t-\frac{\pi}{4}\right)$ & $(60)$ & Flow over infinite flat wall & \\
\hline$\left(\bar{C}_{f, e}\right)_{m}=\frac{3.774}{A_{o}\left(\operatorname{Re}_{\omega}^{0.043}-2.20863\right)}$ & $(61)$ & $15<\operatorname{Re}_{\omega}<400 ; 8<A_{o}<30$ & $\begin{array}{l}\text { (T. S. Zhao and } \\
\text { Cheng 1998a) }\end{array}$ \\
\hline$(f)_{m}=\frac{0.1392}{\operatorname{Re}_{\omega}^{0.25}}$ & $(62)$ & $2800 \sqrt{\omega^{\prime}}\left\langle\operatorname{Re}_{\omega}\right.$ and $4 \leq \sqrt{\omega^{\prime}} \leq 24$ & $\begin{array}{l}\text { (Munekazu } \\
\text { Ohmi, Manabu } \\
\text { Iguchi 1982) }\end{array}$ \\
\hline$(f)_{m}=\frac{0.0791}{\operatorname{Re}_{\omega}^{0.25}}$ & $(63)$ & $\operatorname{Re}_{\omega} \leq 1000$ and $3000 \leq \operatorname{Re} \leq 100,000$ & $\begin{array}{l}\text { (Barreno et al. } \\
\text { 2015; Schlunder } \\
1990)\end{array}$ \\
\hline
\end{tabular}




\subsection{Convective Heat Transfer Correlations}

The correlation for the time-space averaged Nusselt number for air as the working fluid is given by the following relation:

$$
N u=0.00495 A_{o}^{0.9} \operatorname{Re}_{\omega}^{0.656}\left[43.74(D / L)^{1.18}+0.06\right]
$$

Although the present study involves boiling phase change, which renders the problem much more complicated than the single-phase reciprocating flow discussed above, some commonality in heat transfer mechanisms would exist. One of the most important heat transfer mechanisms for both cases is believed to be the boundary layer development/destruction cycle in conjunction with the fluid mixing under a reciprocating condition.

It is commonly known that a boundary layer would be developed as fluid flows in a given direction, e.g., clockwise. As the fluid reverses the direction and flows counterclockwise, the boundary layer developed earlier may be destroyed and a new boundary layer will be developed. This boundary development/destruction cycle will be controlled by the reciprocating frequency of the reciprocating driver. It is expected that as the reciprocating frequency is increased to a specific value, the time-averaged boundary layer will be extremely thin so that the heat transfer coefficient associated with the reciprocating flow would be much higher than that under many other heat transfer modes. 


\begin{tabular}{|c|c|c|c|}
\hline$N u=\alpha W_{0}^{0.2}+\frac{\beta\left(x_{\max } / L_{h}\right) W_{0}^{2} \operatorname{Pr}}{1+\gamma\left[\left(x_{\max } / L\right) W_{0}^{2} \operatorname{Pr}\right]^{2 / 3}}$ & (65) & $\begin{array}{l}W_{0}=\frac{d_{i}}{2} \sqrt{\frac{\omega}{v}} \\
\text { where } \alpha, \gamma \text { and } \beta \text { are constants } \\
\text { depending heating/cooling regions as } \\
\text { prescribed in (Shin and Nishio 1998) }\end{array}$ & $\begin{array}{l}\text { (Shin and Nishio } \\
\text { 1998) }\end{array}$ \\
\hline$N u=1.32 \operatorname{Re}_{\omega}^{0.248} A_{o}^{0.85}$ & (66) & $\begin{array}{l}\operatorname{Pr} \approx 0.7 \\
1000<\operatorname{Re}_{\omega}<4000 \text { and } 7.73<A_{o}<12.88\end{array}$ & $\begin{array}{l}\text { (Akdag and Ozguc } \\
\text { 2009) }\end{array}$ \\
\hline$N u=.0162 \mathrm{Re}_{\omega}^{0.4} A_{o}^{0.85}$ & (67) & $\begin{array}{l}\operatorname{Pr} \approx 0.7 \\
740 \leq \operatorname{Re} \leq 4110 \text { and } 12 \leq \operatorname{Re}_{\omega} \leq 71\end{array}$ & $\begin{array}{l}\text { (Xiao, Chen, et al. } \\
\text { 2014) }\end{array}$ \\
\hline$N u=.02 \operatorname{Re}_{\omega}^{0.58} A_{o}^{0.85}$ & (68) & $\operatorname{Pr} \approx 0.7$ & $\begin{array}{l}\text { (T. S. Zhao and } \\
\text { Cheng 1996b) }\end{array}$ \\
\hline
\end{tabular}




\begin{tabular}{|c|c|c|c|}
\hline & & $23<\operatorname{Re}_{\omega}<464$ and $8.54<A_{o}<34.9$ & \\
\hline $\begin{array}{rl}N u=0 & 0.00495 \operatorname{Re}_{\omega}^{0.656} \\
& \times A_{o}^{0.9}\left[43.74(D / L)^{1.18}+0.006\right]\end{array}$ & (69) & $\begin{array}{l}\operatorname{Pr} \approx 0.7 \\
10<\operatorname{Re}_{\omega}<400 \text { and } 10<A_{o}<35\end{array}$ & $\begin{array}{l}\text { (T. Zhao and Cheng } \\
\text { 1995) }\end{array}$ \\
\hline$N u=1+\sqrt{\operatorname{Pr}}\left(\frac{D}{1+D}\right)$ & (70) & $\operatorname{Re}_{\omega} \rightarrow \infty$ & $\begin{array}{l}\text { (Chen, Luo, and Dai } \\
\text { 2007) }\end{array}$ \\
\hline$N u=0.548 \operatorname{Re}_{\omega}^{0.536} A_{o}^{0.3}$ & (71) & $\begin{array}{l}\text { Rectangular channel, one-sided } \\
\text { heating } \\
43<\operatorname{Re}_{\omega}<684 \text { and } 23<A_{o}<600\end{array}$ & $\begin{array}{l}\text { (Cooper, Nee, and } \\
\text { Yang 1994; T. S. } \\
\text { Zhao and Cheng } \\
\text { 1998b) }\end{array}$ \\
\hline$N u=12.38 \operatorname{Re}_{\omega}^{0.31} A_{o}^{0.95}$ & (72) & $\begin{array}{l}\text { Porous Channel } \\
15 \leq \operatorname{Re}_{\omega} \leq 900 ; 3.1 \leq A_{o} \leq 4 \\
\frac{L}{D_{h}}=3\end{array}$ & $\begin{array}{l}\text { (Leong and Jin } \\
\text { 2005) }\end{array}$ \\
\hline
\end{tabular}




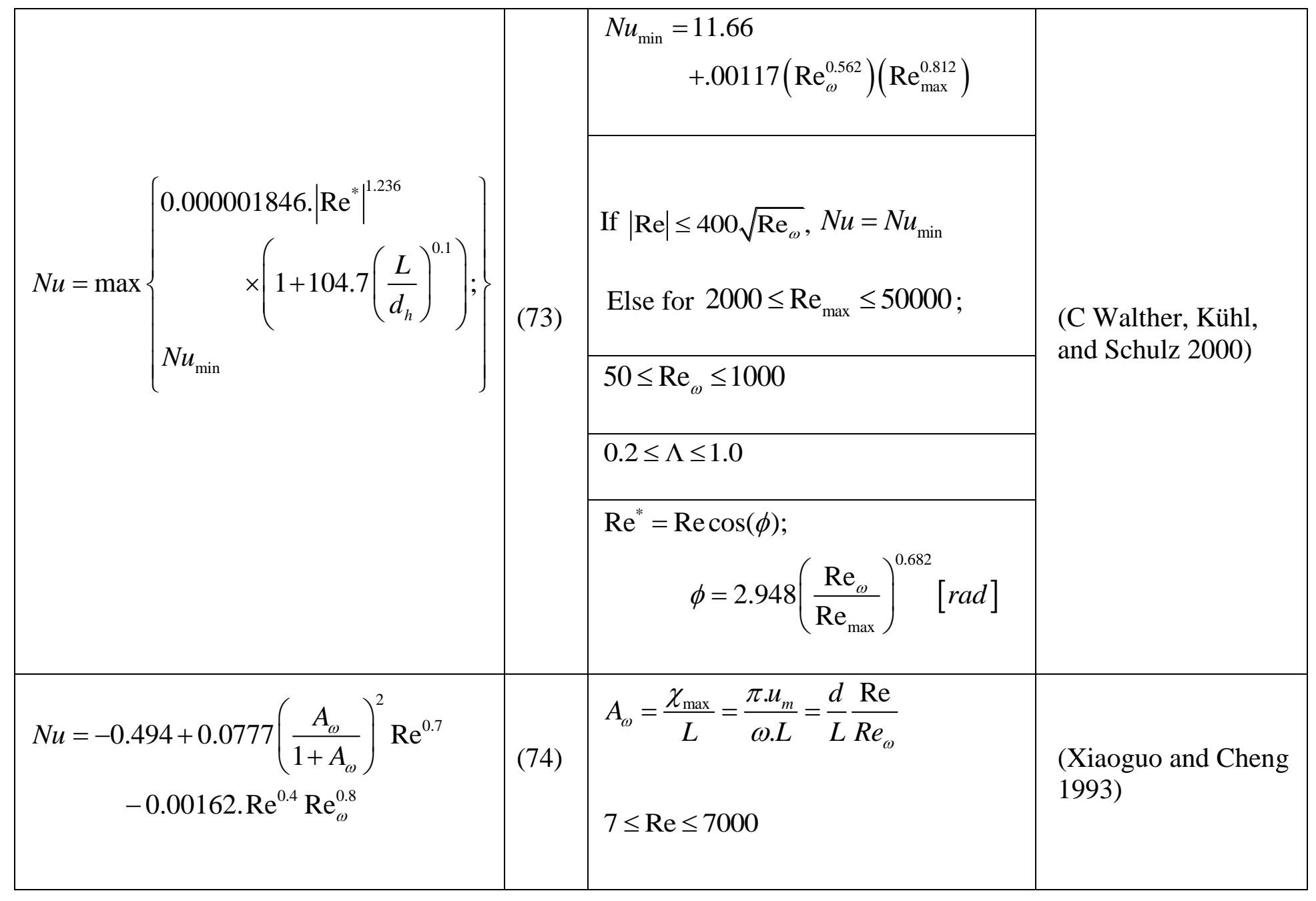




\begin{tabular}{|c|c|c|c|}
\hline & & $\begin{array}{l}7 \leq \operatorname{Re}_{\omega} \leq 180 \\
0.06 \leq A_{\omega} \leq 2.21\end{array}$ & \\
\hline $\begin{aligned} N u=- & 0.494+0.0777\left(\frac{A_{R}}{1+A_{R}}\right)^{2} \operatorname{Re}^{0.7} \\
& -0.00162 . \operatorname{Re}^{0.4}\left(4 \operatorname{Re}_{\omega}^{0.8}\right)\end{aligned}$ & (75) & $\begin{array}{l}A_{R}=\frac{1}{4} \frac{d_{h}}{L} \frac{\operatorname{Re}_{\max }}{R e_{\omega}} ; \operatorname{Re}_{\text {max }}=\frac{u_{\text {max }} d}{v} ; \\
11 \leq \operatorname{Re}_{\max } \leq 10995 ; \frac{d_{h}}{L}=0.0185 ; \\
1.75 \leq \operatorname{Re}_{\omega} \leq 45 ; 0.2 \leq A_{R} \leq 1.13\end{array}$ & (Monte et al. 1996) \\
\hline $\begin{aligned} N u= & 5.78+0.00918 \operatorname{Re}_{\omega}^{0.969} \Lambda^{-0.367} \\
& +0.178\left|\frac{\operatorname{Re}}{\operatorname{Re}_{\max }}\right|^{0.951} \Lambda^{-0.703} \operatorname{Re}_{\omega}^{0.526}\end{aligned}$ & (76) & $\begin{array}{l}\Lambda=\frac{1}{2} \frac{L}{d} \frac{R e_{\omega}}{\operatorname{Re}_{\max }} \\
\operatorname{Re}_{\text {max }}=\frac{u_{\text {max }} d}{v} \\
\operatorname{Re}_{\text {max }}=\frac{u_{\text {max }} d}{v} \\
1000 \leq \operatorname{Re}_{\text {max }} \leq 400 \sqrt{\operatorname{Re}_{\omega}} ;\end{array}$ & $\begin{array}{l}\text { (Ch. Walther et al. } \\
\text { 1998) }\end{array}$ \\
\hline
\end{tabular}




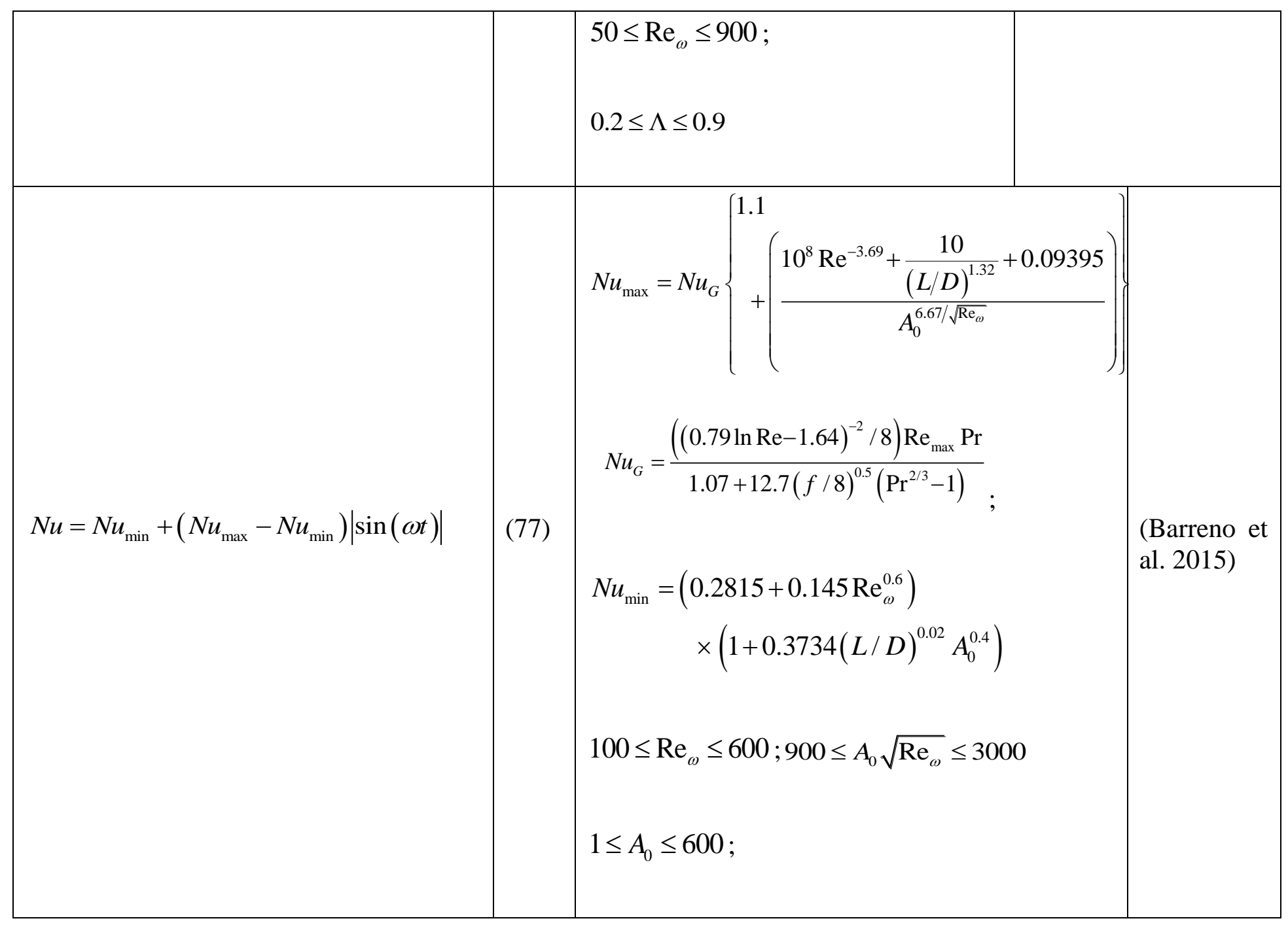




\begin{tabular}{|c|c|c|c|}
\hline$N u=0.372 \operatorname{Re}_{\omega}^{0.78}$ & (78) & $\begin{array}{l}\text { vertical annular channel } \\
A_{0}=\frac{L}{D}=7.73 \\
\operatorname{Pr}=4.65 \\
1000 \leq \operatorname{Re}_{\omega} \leq 4000\end{array}$ & $\begin{array}{l}\text { (Akdag, Ozdemir, and } \\
\text { Ozguc 2008) }\end{array}$ \\
\hline$N u=0.021 \overline{\operatorname{Re}}_{s c}^{0.8} \operatorname{Pr}_{o}^{0.4}\left(\frac{T_{w}}{T_{b}}\right)^{-0.5} . C^{\prime}$ & (79) & $\begin{array}{l}C^{\prime}=0.923+0.750\left(\frac{T_{w}}{1000}\right) \\
\operatorname{Re}_{s c}: \text { Reynolds number (based on } \\
\text { Schmidt cycle model (Kanzaka and } \\
\text { Iwabuchi 1992)) } \\
\operatorname{Pr} \approx 0.7 ; \\
10\left\langle\operatorname{Re}_{\omega}<400 \text { and } 10<A_{o}<35\right.\end{array}$ & $\begin{array}{l}\text { (Kanzaka and } \\
\text { Iwabuchi 1992) }\end{array}$ \\
\hline
\end{tabular}




\begin{tabular}{|c|c|c|c|}
\hline $\begin{aligned} N u= & 0.0035 \operatorname{Re}_{n}^{1.3} \operatorname{Pr}_{o}^{113} \\
& +0.3\left(\frac{\operatorname{Re}_{o}^{2.2}}{\left(\operatorname{Re}_{n}+800\right)^{1.25}}\right)\end{aligned}$ & (80) & $\begin{array}{l}100<\operatorname{Re}_{n}\langle 1200 ; \\
\operatorname{Re}_{n}=\frac{U D}{v} \\
\operatorname{Re}_{o}=\frac{x_{\max } \omega D}{v}\end{array}$ & $\begin{array}{l}\text { (Mackley and } \\
\text { Stonestreet 1995) }\end{array}$ \\
\hline$N u=0.4 P e+0.315$ & (81) & $\begin{array}{l}5.1<P e<16.6 \\
40<\operatorname{Re}_{\max }<240\end{array}$ & \\
\hline$N u=0.71 \operatorname{Re}^{0.38} \operatorname{Pr}^{0.4}$ & (82) & $\begin{array}{l}61.7<\operatorname{Re}<265.9 \\
\text { U shaped tube }\end{array}$ & (Ni et al. 2015) \\
\hline$N u=0.71 \operatorname{Re}^{0.47} \operatorname{Pr}^{0.4}$ & (83) & $448.5<\operatorname{Re}<3184.6$ & \\
\hline$N u=\sqrt{\frac{P e_{\omega}}{2}} \frac{[(1+i) \tanh z]}{[(1+i) \tanh z]}$ & (84) & $z=(1+i) \sqrt{\frac{P e_{\omega}}{32}} ;$ & $\begin{array}{l}\text { (Grassmyer } \\
\text { Cheng 1995) }\end{array}$ \\
\hline
\end{tabular}




\begin{tabular}{|c|c|c|c|}
\hline & & $P e_{\omega}=\frac{\omega D^{2}}{\alpha}$ & \\
\hline$N u_{r}=N u_{i}=0.56\left(P e_{\omega}\right)^{0.69}$ & (85) & $\begin{array}{l}100 \leq P e_{\omega} \leq 10,000 \\
q_{c} "=\frac{k}{D_{h}}\left[N u_{r}\left(T_{c}-T_{w}\right)+\frac{N u_{i}}{\omega} \frac{d T}{d t}\right]\end{array}$ & (Grassmyer 1994) \\
\hline$N u=-\frac{2 \delta}{k T_{w}}\left(-I_{1} \cos t+I_{2} \sin t\right)$ & (86) & $\begin{array}{l}I_{1}=\frac{\alpha-\gamma_{R}}{2}- \\
\quad \frac{\operatorname{Pr}}{2}\left(\frac{-\alpha+\gamma_{i} \alpha+\left(\alpha+1-\gamma_{R}\right)(1-\operatorname{Pr}+\alpha)}{\alpha^{2}+(1-\operatorname{Pr}+\alpha)^{2}}\right) \\
\gamma_{R}+\gamma_{i}=\alpha^{4} \sqrt{1+R_{\omega}^{2} \operatorname{Pr}^{2}(\cos \theta+i \sin \theta)} \\
\theta=R_{\omega} \operatorname{Pr} \\
\text { Oscillatory flow with sinusoidal wall } \\
\text { temperature }\end{array}$ & (Ramos et al. 2004) \\
\hline
\end{tabular}




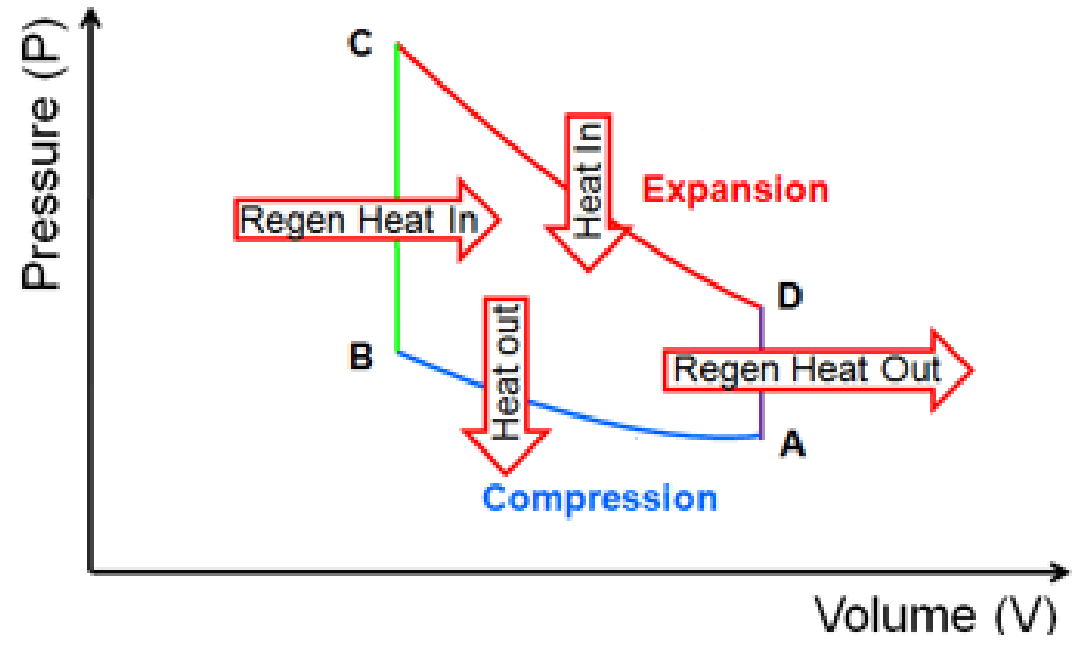

(a)

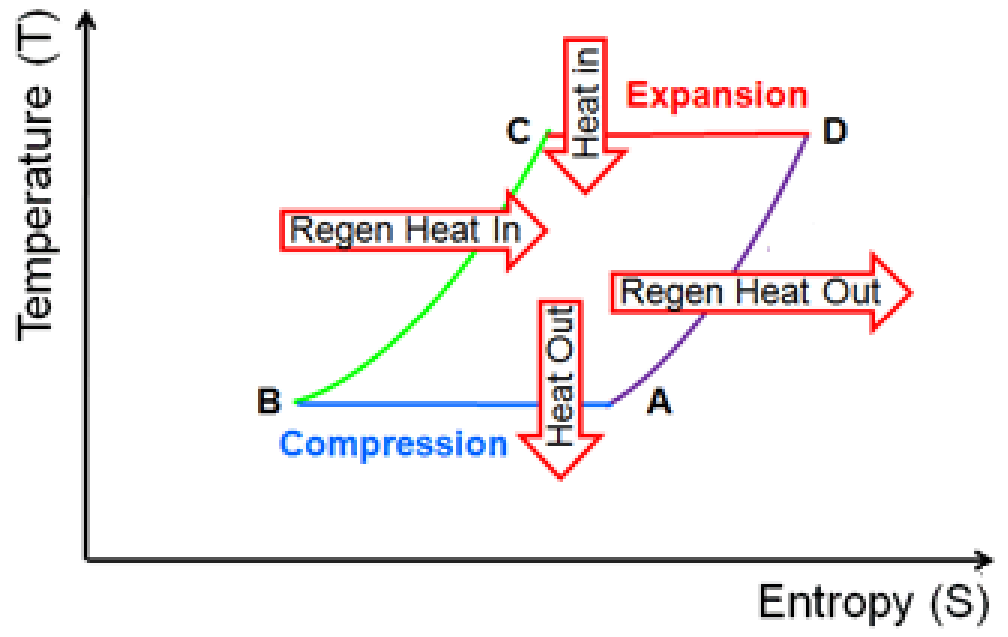

(b)

Figure 2. 13: Thermodynamic cycles for Oscillating cooling (a) P-v (b) T-S (Woodbank Communications Ltd 2005; Tziranis 1992) 


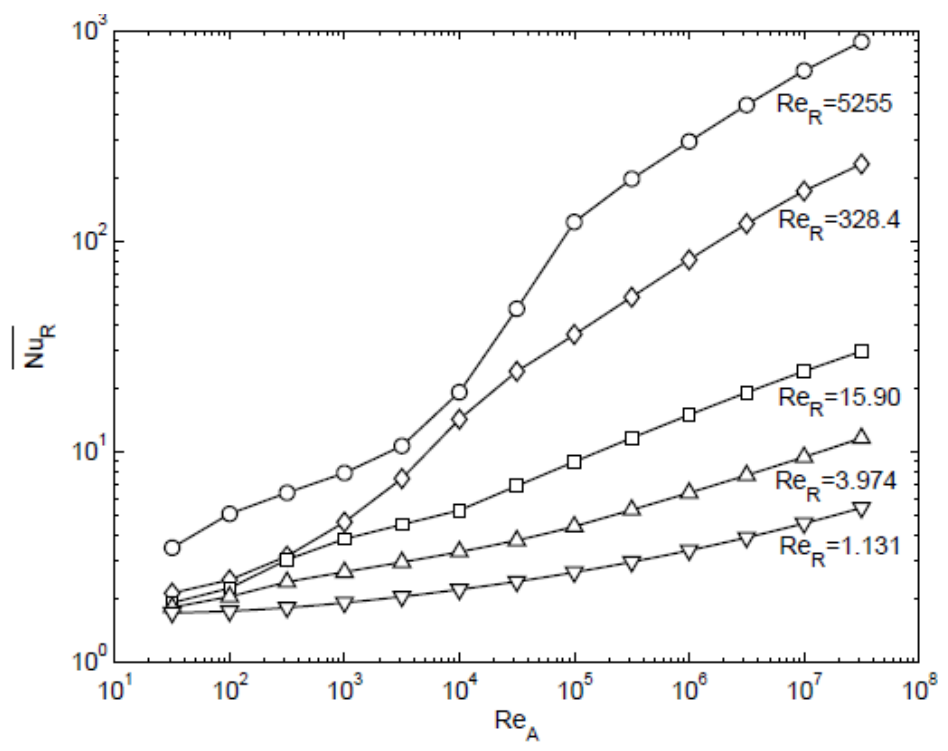

(a)

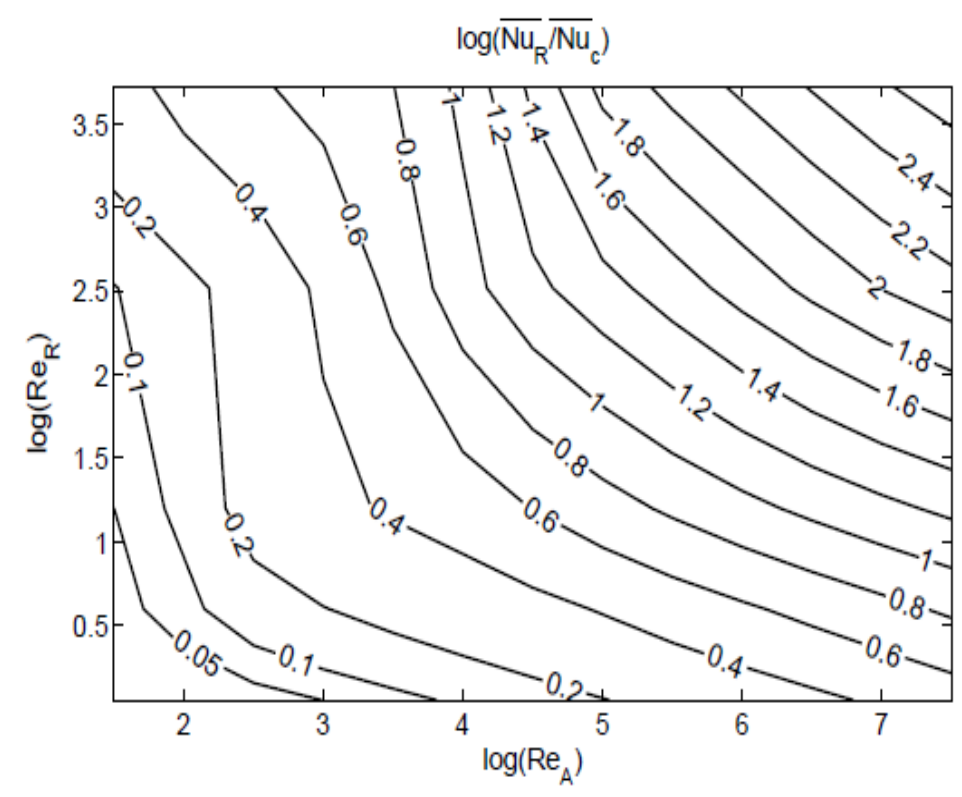

(b)

Figure 2. 14 : Contour plot of Nusselt Number (Su, Davidson ${ }_{N u_{r}}^{\text {and }}{ }_{N} u_{i}$ lacki 2011)

(a) Averaged $N u_{r}$ for various cases (b) Contour plot of ratio of real 
Though the configuration of the RMDL is simple, the analysis of the thermodynamics of it operation, the fluid dynamics of its two-phase oscillating flow and sensible and latent heat transfer is quite complicated (O. Popoola and Cao 2016). Eq.(65) to Eq. (86) provides the most applied $N u$ Correlations for oscillating pipe flow. In addition, these correlation Fig. 2. 14 has also been proposed as an alternative prediction for $\mathrm{Nu}$. These correlations were obtained from the experimental analysis; some were Analytical. The analytical correlations include the complex Nusselt number $N u_{i}$ correlations proposed by the researchers from Massachusetts Institute of Technology (MIT). 


\section{CHAPTER THREE: EXPERIMENTAL STUDIES}

Like many other heat transfer studies, the experimental determination of the heat transfer coefficient in the reciprocating flow is one of the most important fundamental research topics, which would provide practical design tools for commercial heat transfer devices involving the RMDHL. Also important is the issue of performance data of the RMDHL which will serve as a benchmark for numerical models. The specific objectives of the experimental investigations of this study are as follows:

1. Measurement of bulk temperature of working fluid within the RMDHL loop under varying heat loads.

2. Evaluation of characteristic cold plate temperature under varying operational conditions of the RMDHL.

3. Varying the operational parameter of the working loop to develop further understanding of the relationships between dependent variables.

4. Generating performance data that will be used to validate Numerical Models.

5. Dimensional analysis /correlation for single phase flow.

Extensive literature review presented in the previous chapter has identified the issue associated with heat transfer correlations for devices like the RMDHL that achieves cooling with oscillating flow. This study believed that the heat transfer rate in the RMDHL is affected by the piston stroke of the reciprocating driver, piston 
reciprocating frequency, liquid filling ratio, size of the heat loop including the inner diameter of the liquid reservoir and the diameter of the loop tubing, working fluid of the loop, and orientation of the loop relative to the gravitational field, which may be expressed by Eq. (1) below:

$$
q=F\left(D, \omega, A, L, \nu, x, y, z, g, \alpha, \rho, \nu, c_{p}, k, T, i\right)
$$

Because of the large number of dimensional variables, nondimensionalized equations with dimensionless numbers are adopted (O. Popoola, Soleimanikutanaei, and Cao 2016b). Two dimensionless equation structures were considered in this study. The first is of an exponential form derived based on dimensional analysis and similitude (Fox, McDonald, and Pritchard 2009; Bergman, Lavine, and Incropera 2012):

$$
N u=\frac{h_{r c} D_{h}}{k_{l}}=C A_{o}^{n_{1}} \operatorname{Re}_{\omega}^{n_{2}}\left(D_{h} / L\right)^{n_{3}} \operatorname{Pr}^{n_{4}}
$$

where $C, n_{1}, n_{2}, n_{3}$, and, $n_{4}$ are constants to be determined from experimental data, $D_{h}$ is the hydraulic diameter of the flow channel, $L$ is the heated length, and $\operatorname{Re}_{\omega}, A_{o}$, and Pr are, respectively, the kinetic Reynolds number, the dimensionless fluid displacement, and Prandtl number, as defined by Eqs. (3-5):

$$
\begin{gathered}
\operatorname{Re}_{\omega}=\frac{\omega D^{2}}{v} \\
A_{o}=x_{\text {max }} / D \\
\operatorname{Pr}=\frac{c_{p} \mu}{k}
\end{gathered}
$$

The dimensionless oscillation amplitude of the fluid in Eq. (4) is equivalent to the dimensionless fluid tidal displacement of Cooper and Yang (1994). 
The second structure was to use the form proposed by (Shin and Nishio 1998) as follows:

$$
\begin{aligned}
& \overline{N u}=\alpha W_{o}^{n_{1}}+\frac{\beta\left(A / L_{e}\right) W_{o}^{n_{2}} \operatorname{Pr}}{1+\gamma\left[\left(A / L_{e}\right) W_{o}^{n_{2}} \operatorname{Pr}\right]^{n_{3}}} \\
& W_{o}=\sqrt{\operatorname{Re}_{\omega}}
\end{aligned}
$$

where $\alpha, \beta, \gamma, n_{1}, n_{2}$, and $n_{3}$ are constants to be determined from experimental data.

The experimental investigations of this study were therefore conducted under the following three specific categories:

1. Bulk temperature measurements during single phase operation of the RMDHL

2. Bulk temperature measurements during two-phase operation of the RMDHL

3. Dimensional analysis to determine heat transfer correlations

\subsection{Design and Construction of a Single-Phase Testing Rigs}

The early development of the RMDHL had involved two main prototype configurations. The first is the solenoid type (Cao and Gao 2008) and the second is the bellows type (Cao, $\mathrm{Xu}$, and Gao 2013). The solenoid type RMDHL, designed for higher heat flux two phased cooling, features a smaller sized evaporator, and the reciprocating driver assembly consist of a solenoid based magnetic system. The bellows type RMDHL on the other hand is designed for high heat transfer rates, single phase operation, and a reciprocating driver assembly designed to incorporate bellows. The single phase experimental tests were conducted using the bellows type RMDHL. 
Photos of assembled bellows type heat loop developed prior to this study, referred to as first generation prototypes, is shown in Fig. Error! Reference source not found. and Fig 3.2. Fig 3.3 presents a photo of the second-generation rig developed at the early stages of this study, where the $\mathrm{X}$ rack in the first-generation configuration has been replaced by a 'twin bellows pump assembly'. The third-generation experimental rig developed for investigate heat transfer correlations and reduce issues associated with minor pipe loss within the heat loop is presented in Fig 3.8. All of the configurations consist of an evaporator, a condenser, and a reciprocating driver assembly. The size of evaporator, condenser and the connecting tubing are selected based on the maximum thermal load of the target experiment. As illustrated inn Fig 3.1 and Fig 3.2, the condenser in Fig 3.1 is about $10 \%$ of the condenser in the configuration presented in Fig 3.2.

The prominent feature of the first-generation prototype is the ' $\mathrm{X}$ rack bellows stand' which was constructed (and is schematically shown in Fig 3.1b and Fig 3.2) to fascilitate the assembling of the bellows and reciprocating driver. As seen in the figures, two bellows are placed side by side in a bellows housing. A partition plate between the two bellows holds the two bellows together. The other sides of the two bellows are fixed to the housing plates, respectively, on the frontal and rear sides of the bellows housing. The partition plate is connected to the reciprocating driver through a four-arm rack and four connecting rods that run through the frontal housing plate. During the operation, the reciprocating motion of the driver arm generates a reciprocating motion of the partition plate, which could then 


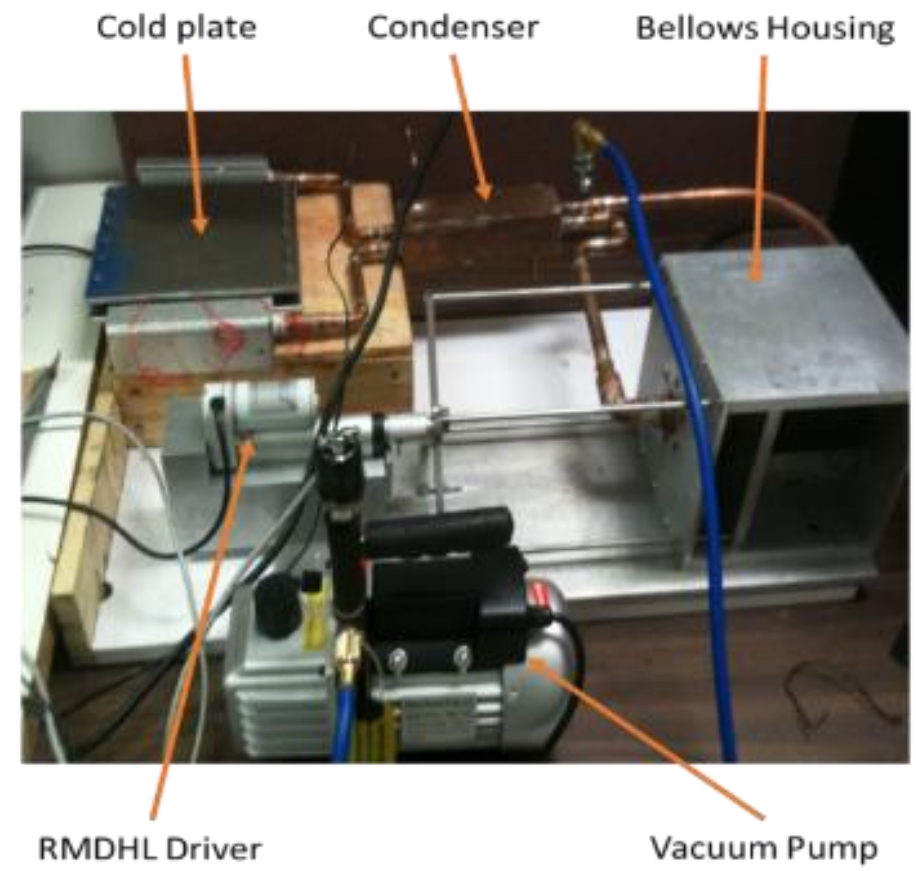

(a)

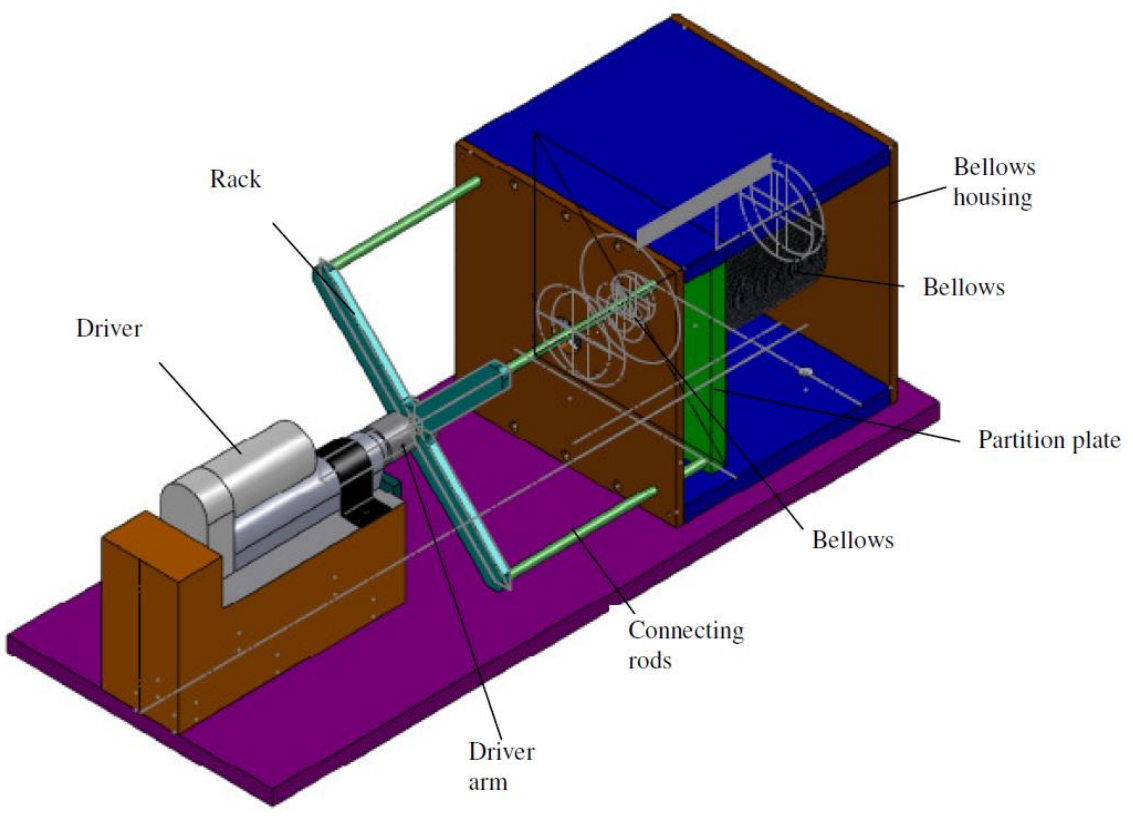

(b)

Fig. 3. 1: First generation bellow type RMDHL for Single phase high heat rate operation(Cao, $\mathrm{Xu}$, and Gao 2013) (a) A photo of the assembled bellows-type heat loop being evacuated (b) Configuration of the designed bellows/driver assembly. 


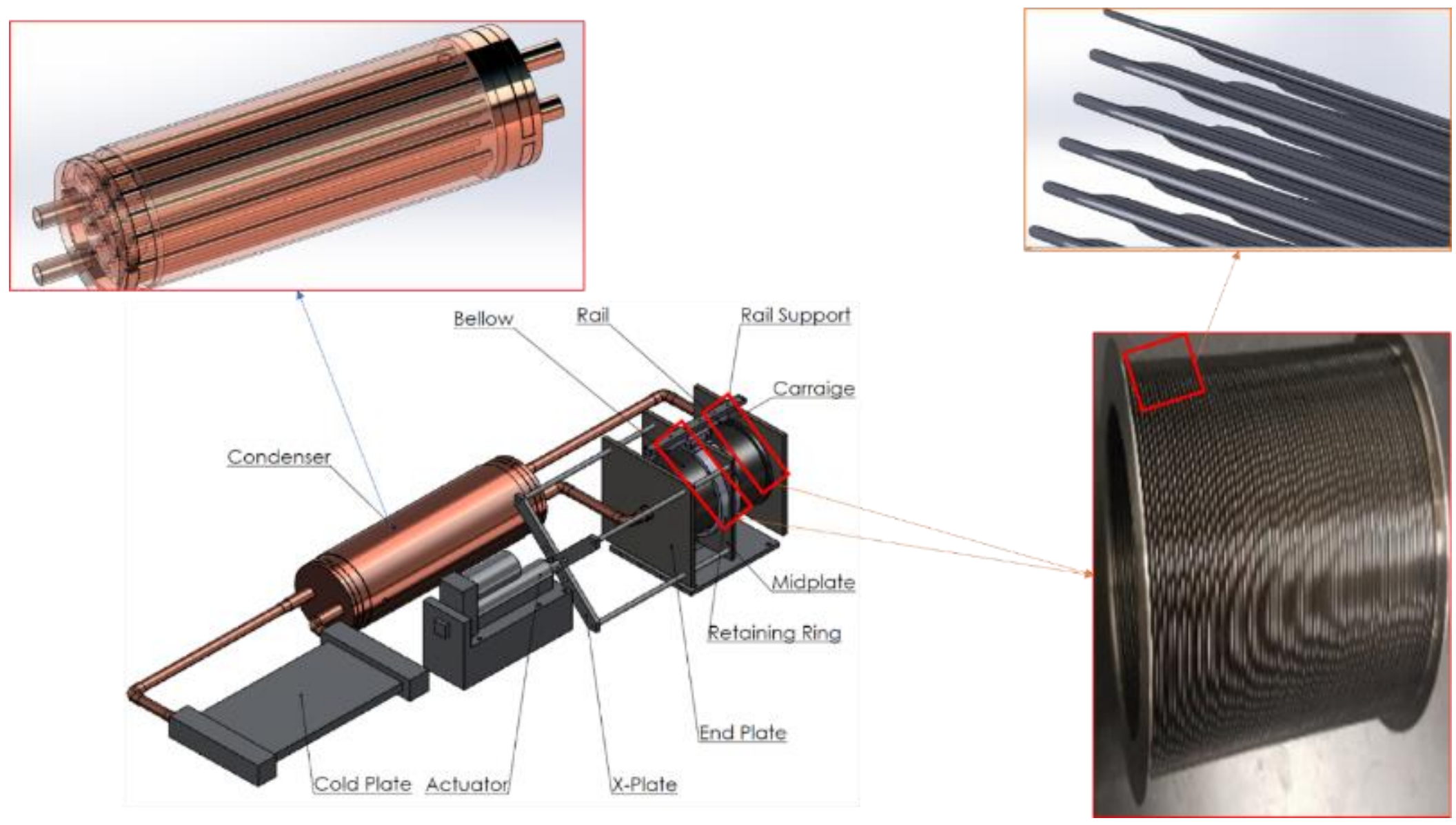

Fig. 3. 2: Alternate configuration of First generation RMDHL with a condenser designed for higher cooling loads for single phase high heat flux cooling 


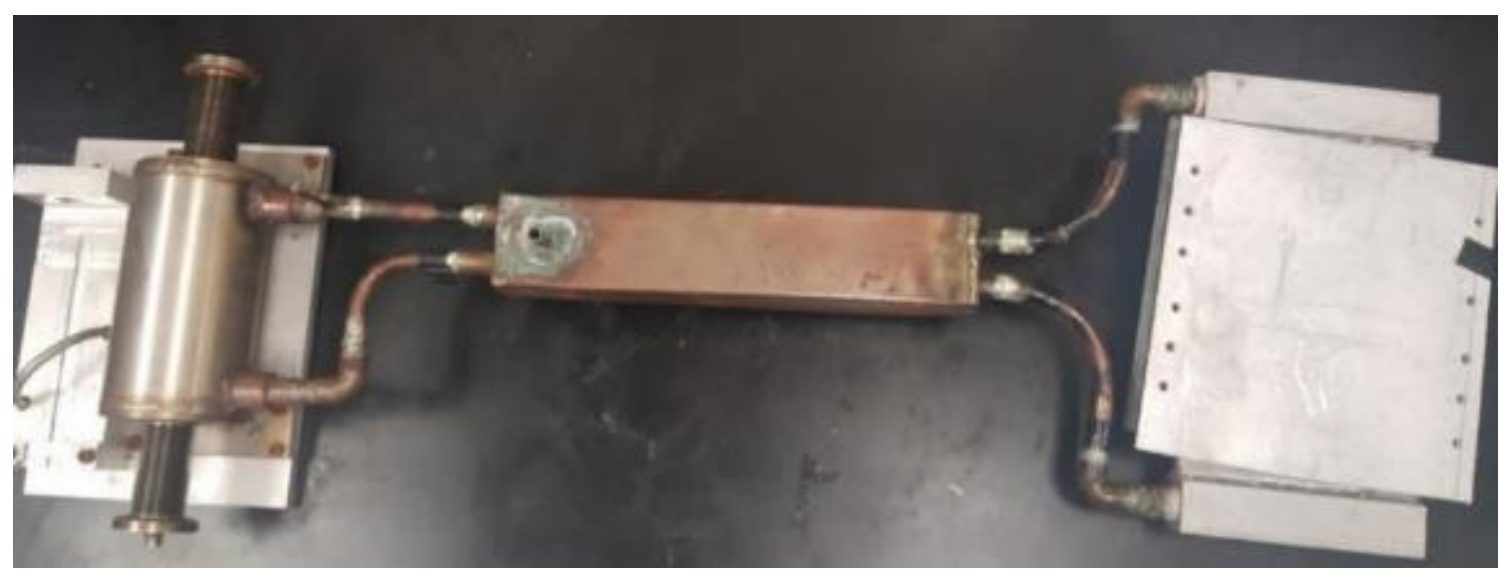

(a)

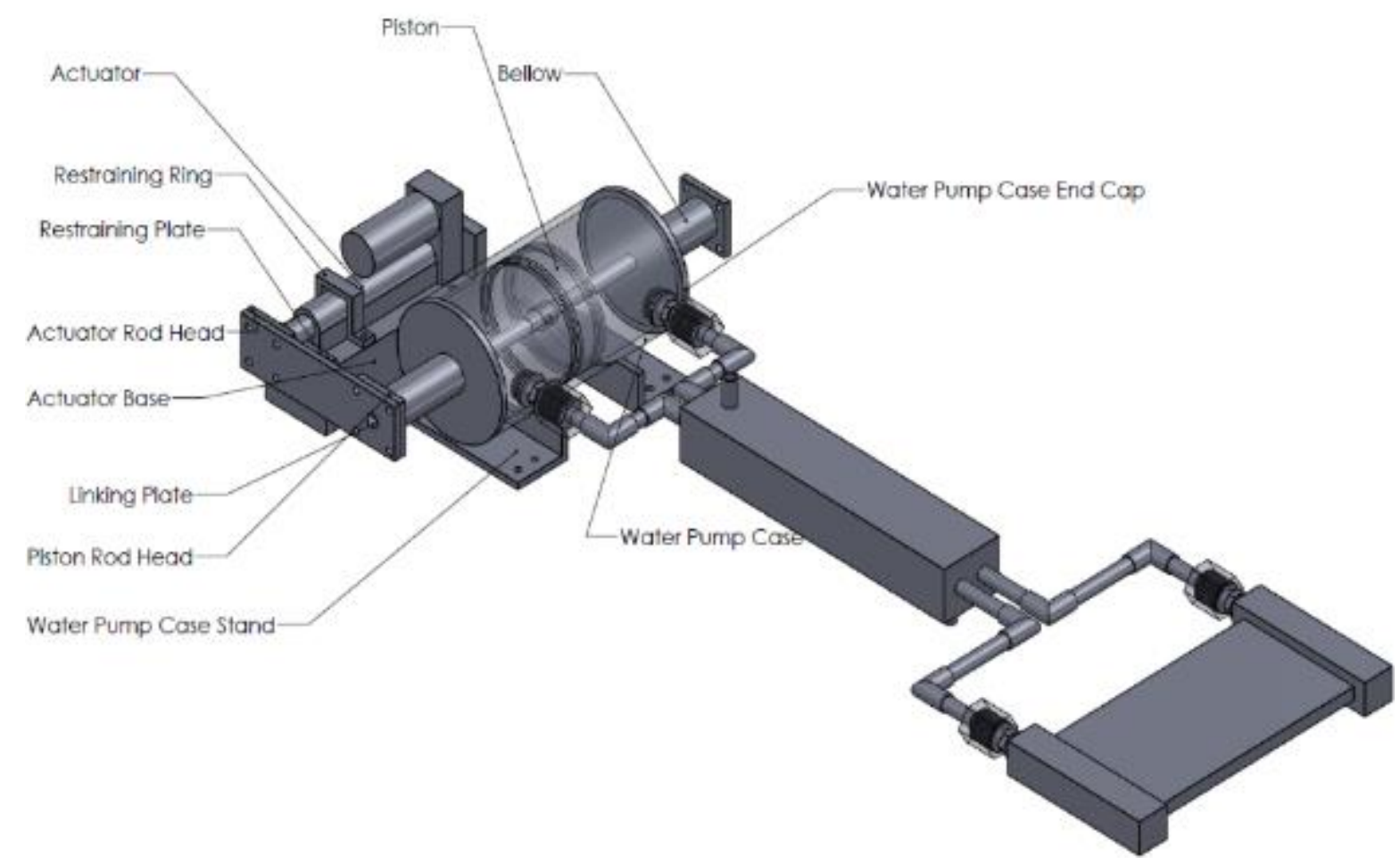

(b)

Fig. 3. 3: Second generation bellow type RMDHL for Single phase high heat rate operation (a) A photo of the assembled second-generation bellows-type heat loop without the RMDHL driver (b) Schematic drawing of the second generation RMDHL assembly. 
create a reciprocating motion of the working fluid hermetically enclosed within the bellows type RMDHL. The two-bellows used are metal welded bellows of $8.30 \mathrm{~cm}$ OD $\times 5.70 \mathrm{~cm}$ ID with 50 convolutions, which could accommodate a reciprocating stroke of $10 \mathrm{~cm}$. The reciprocating driver is a mini actuator having a maximum stroke of 7.62 $\mathrm{cm}$.

In the second and third generation prototypes, the partition plate is replaced by a piston which is enclosed in a pump assembly. The pump assembly features twin bellows which are attached to each ends of the pump. The pump is then connected to the reciprocating driver via a linking plate. These design improvement, developed during the current study, is intended to reduce the fatigue loading on the bellows and to provide a sturdier finish (when compared to the first generation configuration) and leak proof RMDHL driver. The bellow in this configuration also have 50 turns of 4.30 $\mathrm{cm} \mathrm{OD} \times 2.70 \mathrm{~cm}$ ID. All setups includes electric heaters for supplying heat to the cold plate of the heat loop, a DC power supply to the reciprocating driver, a control circuit board to control the reciprocating frequency of the driver, a constant temperature circulator to maintain a constant coolant inlet temperature to the condenser, which would also control the operating temperature level of the heat loop, a data acquisition system, and the fabricated heat loop. The portion of the heat loop from the evaporator (cold plate) to the condenser was placed in a horizontal position to eliminate the influence of gravity on the performance of the heat loop.

The head loss within the loop is regarded as the sum of the major loss losses due to frictional effects in straight pipe flow, and the minor losses resulting from 
entrances, fitting, area changes and so on. Depending on the device, minor losses traditionally are computed in one of two ways, either by using the loss coefficient or by using the equivalent length of the straight pipe. Either way, coefficients must be determined experimentally for both methods. For oscillating flow, many of these coefficients are unavailable. The third generation RMDHL configuration developed in this study eliminates the use of elbows, connectors, abrupt bends and corners. This design reduces the variability which may result from minor losses, and also reflects modern technological trends in cooling loop like the ribbon shaped Tesla cooling tubes (Hermann et al. 2012). The evaporator, condenser and connecting tubes are formed from a continuous copper pipe tubing, and parker compression fittings were used to eliminate the need for welding and to enhance reconfigurability. A photo of the loop configuration is presented in Fig 3.4. The figure is before the loop is attached to the RMDHL driver. The completely assembled configuration is presented in Fig 3.8.

The working fluid used for the heat loop is water because of the lower pressure inside the loop. After the evacuation of the heat loop, a total amount of $700 \mathrm{cc}$ of water is charged into the heat loop, which represents approximately a $70 \%$ charge ratio based on the total interior volume of the heat loop. Water was also used as the cooling fluid in the condenser loop. 


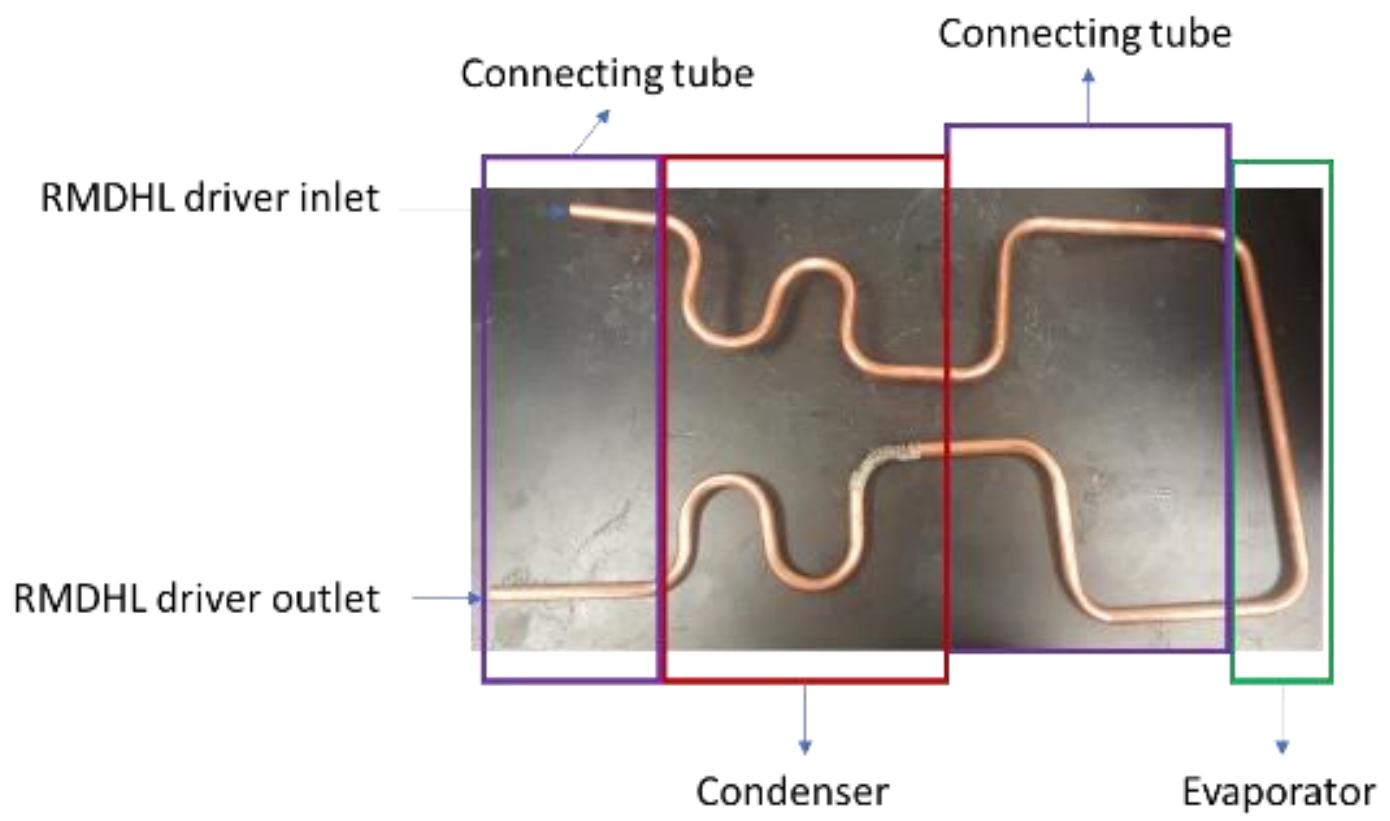

Fig. 3. 4: Photo of the Evaporator, condenser and connecting tube configuration adopted in third generation prototype 


\subsubsection{Evaporator design for bulk temperature measurements}

The rectangular shape of the evaporator (Fig 3.5) had been selected for the prototype development because it reflects the module arrangement in the typical power pack of an EV running with Li-ion cell. As seen in the illustration of the power pack in the Tesla Model S in Fig. 3.6 and Fig. 3.7, the rectangular cell pack in the Model S features 16 modules with 444 cells that are also in a rectangular array. The module arrangement features a ribbon filled with glycol (similar to rectangular channels) running parallel to the cells for thermal management.

For the evaporator in the experimental rig, eight Omega flexible heaters (four on each side of the cold plate) are clamped by two aluminum plates onto the cold plate with insulation layers sandwiched between the heaters and clamping plates. Each heater has a dimension of $2 \mathrm{~cm}$ by $12 \mathrm{~cm}$ and could provide a heat input up to $200 \mathrm{~W}$. Twelve thermocouples were placed at different locations of the heat loop. Nine of them (numbered as No. 1 through No. 9) are placed on the top surface of the cold plate at different locations for the study of temperature uniformity on the cold plate. Fig $3.5 \mathrm{~b}$ shows exact locations of these thermocouples. One thermocouple is placed near the condenser, and another two thermocouples are placed on two ends of the bellows pump to monitor the heat loop temperature distribution in addition to those on the cold plate.

The maximum fluid displacement that could be provided by the bellows/driver combination is approximately $285.0 \mathrm{cc}$. This is compared to the total interior volume of the cold plate of $186 \mathrm{cc}$ including those of both grooves and manifolds. Fig. 3.5a shows the constructed cold plate having an overall dimension of 5.91 " $(15 \mathrm{~cm}) \times 11.81$ " 

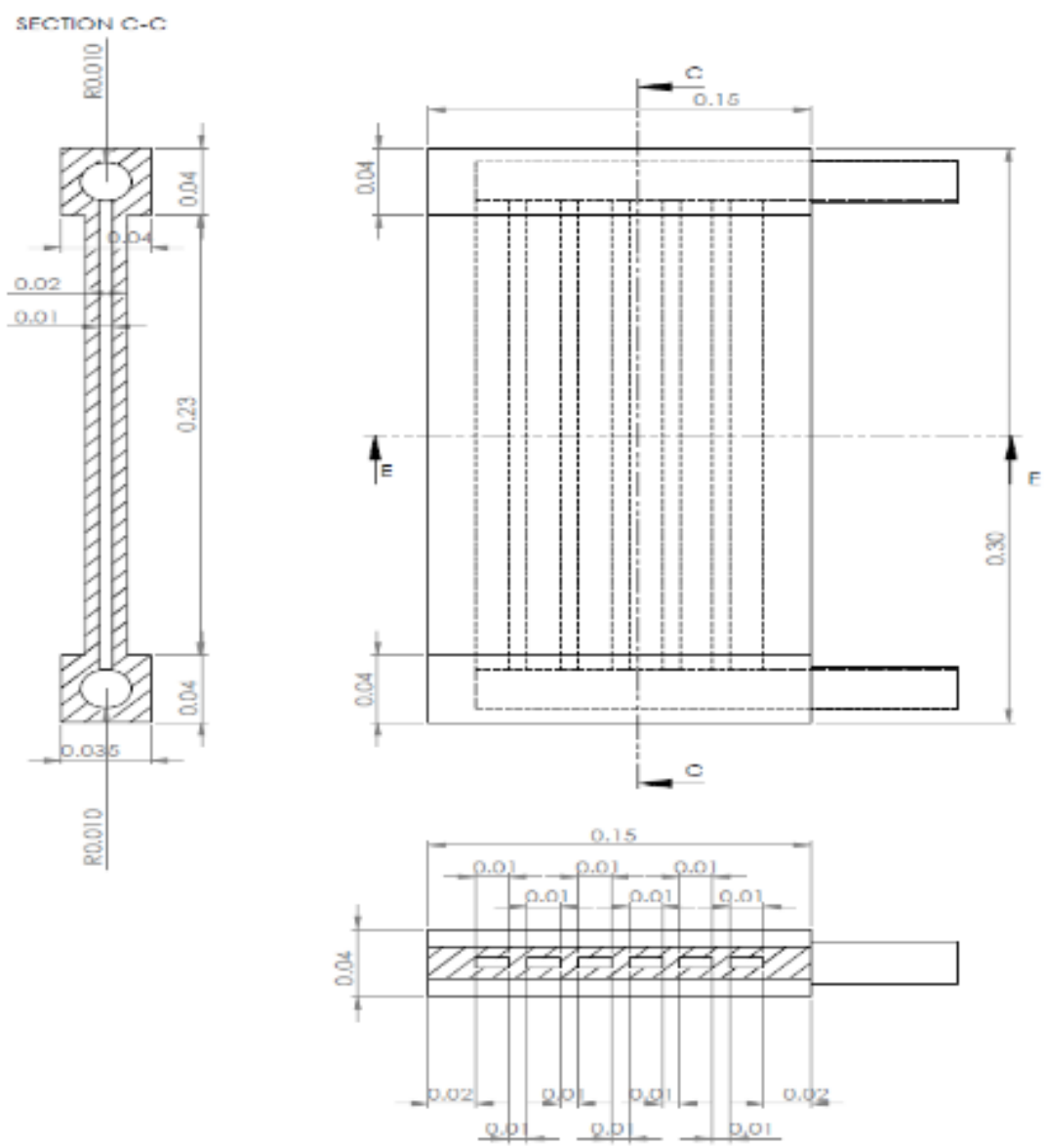

SECTION E-E

(a)

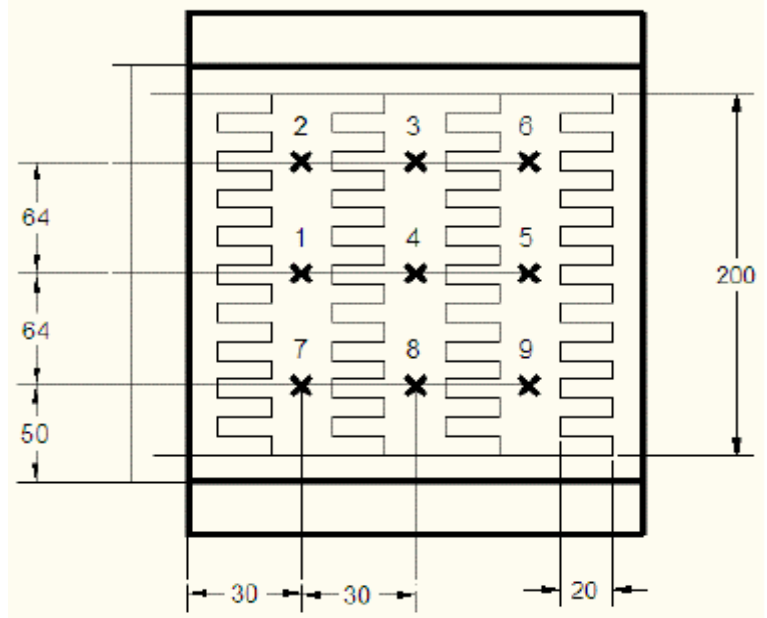

(b)

Figure 3. 5: (a) Physical dimensions of the cold plate and (b) thermocouple locations on the cold plate (size in mm) (Cao, Xu, and Gao 2013). 


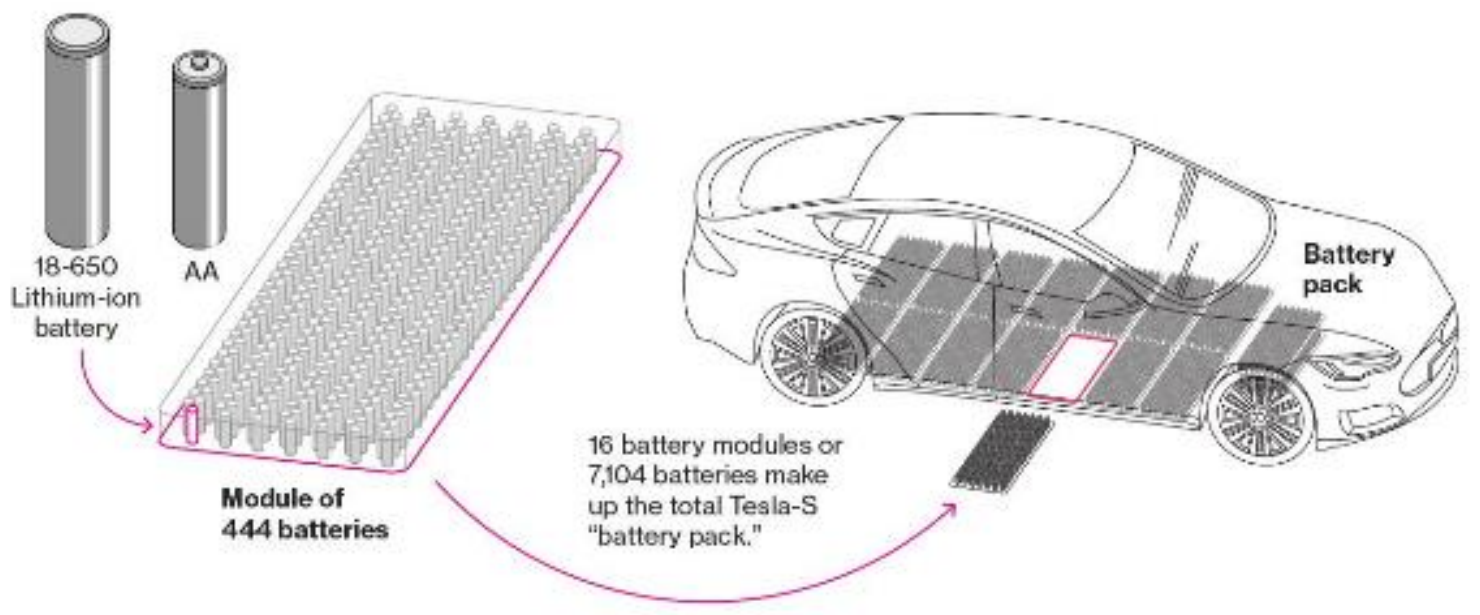

Figure 3.6: Battery, Module and Pack arrangement in the Tesla Model S

(Shankleman et al. 2017)

Ribbon Shaped Tesla patent cooling tube 18650 battery cells

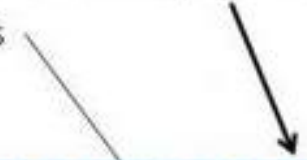

Glycol coolant in

000000000000000000000000000000

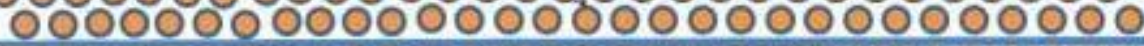

00000000000000000000000000

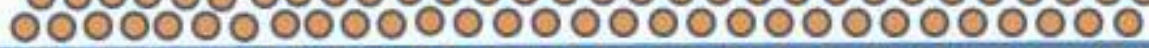

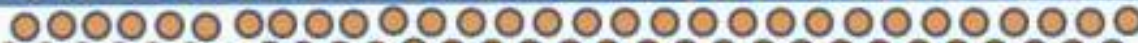

000000000000000000000000000

000000000000000000000000000 000000000000000000000000000

000000000000000000000000000000 ००0000000000000000000000000

उ000000000000000000000000 000000000000000000000000000

0000000000000000000000000000

000000000000000000000000

Glycol coolant out

Figure 3. 7: Cooling configuration for the battery module in Tesla model $\mathrm{S}$ electric vehicle (Steve Hanley 2016) 
$(30 \mathrm{~cm})$ (top left). The cold plate has two circular manifolds of 0.79 " $(2 \mathrm{~cm})$ on both top and bottom of the plate, respectively (top right). A total of six grooves are machined from the cold plate, running through the cold plate and communicating with both manifolds. The grooves provide flow passage for reciprocating two-phase flow, and have a cross-sectional area of $0.20 "(0.51 \mathrm{~cm}) \times 0.51 "(1.30 \mathrm{~cm})$, as shown in the bottom drawing of Fig 3.5.

\subsubsection{Surface/volume requirement in the condenser section}

The condensers built for each of the experimental rig is similar to a shell/tube heat exchanger with the working fluid of the RMDHL flowing inside the tubes and the water coolant from the chiller circulator flowing outside of the tubes. For geometric

considerations, the two (or more) condensers on both sides of the bellows pump are housed within the same shell with a shell partition between them. The overall heat transfer coefficient may be written as

$$
\frac{1}{A_{c} U_{c}}=\frac{1}{A_{h} U_{h}}=\frac{1}{(h A)_{c}}+R_{w}+\frac{1}{(h A)_{h}}
$$

where $c$ and $h$ refer to the chiller fluid and working fluid in the loop, respectively, $U$ is the overall heat transfer coefficient, $h$ is the heat transfer coefficient, $A$ is the surface area, and $R_{w}$ is the thermal resistance related to the container walls. 


\subsection{Experiment Setup for Single-Phase Heat Transfer Correlation}

As discussed in chapter 2 , there is a significant degree of disparity in the $\mathrm{Nu}$ correlations adopted in various studies of convective heat transfer in which the cooling fluid is a mechanically driven oscillating flow. These inconsistencies have been well documented and were linked to inconsistencies experienced in the analysis during the early stages of the current studies. It was discovered in the course of the current study that existing correlations typically underpredict the $N u$ for the RMDHL or are completely inaccurate (O. T. Popoola and Cao 2015; O. Popoola, Bamgbade, and Cao 2016). In line with the objective of the current investigation to generate suitable correlations for the single-phase reciprocating forced convection, $h_{r c}$, the procedure adopted for achieving this objective are discussed in the following sections.

The dimensionless equation structure presented in Eq. 6 was selected as the working formulation for defining the Nu correlation. This is because unlike Eq. 2, Eq. 6 presents the direct relation to piston stroke in conjunction with the inner diameter.

The piston stroke in conjunction with the inner diameter of the reservoir is believed to be one of the most critical operational parameters because it would determine the oscillation amplitude of the working fluid and the rewetting capability of the liquid to reach from the condenser section to the evaporator section. The experimental rig was developed using the calculations presented in chapter 5 and the methodology is similar to the description in the preceding sections of chapter 3 . Fig 3.8 and Fig. 3.9 present the schematic of the experimental test rig. In the present study, the 
piston stroke was maintained between 30 to $76 \mathrm{~mm}$. The piston reciprocating frequency had a favorable effect on the sweeping speed of the fluid over the heated surface.

Another important variable in Eq. 6 that was expected to have a significant impact on the performance of the RMDHL loop is the distance between the evaporator and the condenser, $\Delta x$. In practical cooling application, $\Delta x$ determines the geometry of the design allowing for the cold sink to be located in the most effective heat dissipation region even if this region is at a significant distance from the location of heat generation.

Take the example of an electric vehicle (EV), where a pack of batteries is composed of modules and placed in a single container (the equivalent of our evaporator) for thermal management (Fig 3.6). An EV may have more than one pack of battery situated in a different location in the car (Young et al. 2013). The radiator on the other hand is located under the hood towards the front end of the car. The experimental rig is designed to facilitate a prototype length of up to 1 meter between the condenser and the evaporator. The location of the evaporator is fixed but the condenser was located at $15 \mathrm{~cm}, 30 \mathrm{~cm}$, or $60 \mathrm{~cm}$ from the evaporator as demonstrated in Fig 3.9.

An Omega polyimide film insulated flexible heater with a width $2.5 \mathrm{~cm}$ (1 in) and length $1.3 \mathrm{~cm}(0.5 \mathrm{in})$ provided heat input to the evaporator section. The $4 \times 6 \times 8$ inches condenser was constructed from heat resistant acrylic plastic. The coolant in the condenser is water, and the coolant inlet temperature was kept at various temperatures using the constant-temperature circulator. The loop has an interior pipe diameter of 

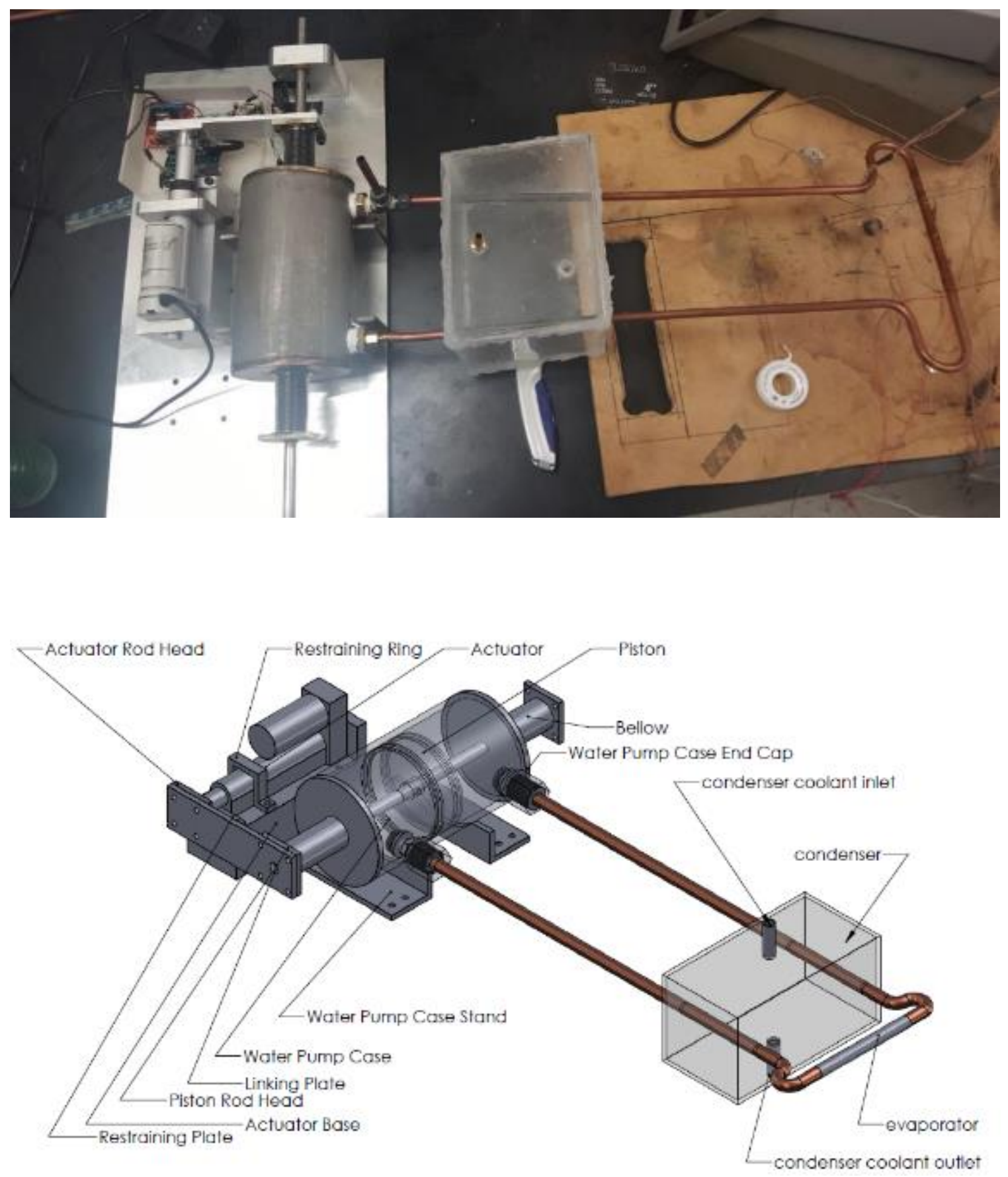

Figure 3. 8: (a) Picture of Configuration of an experimental setup to characterize RMDHL for single phase correlation (b) Schematic drawing of the third generation RMDHL assembly 


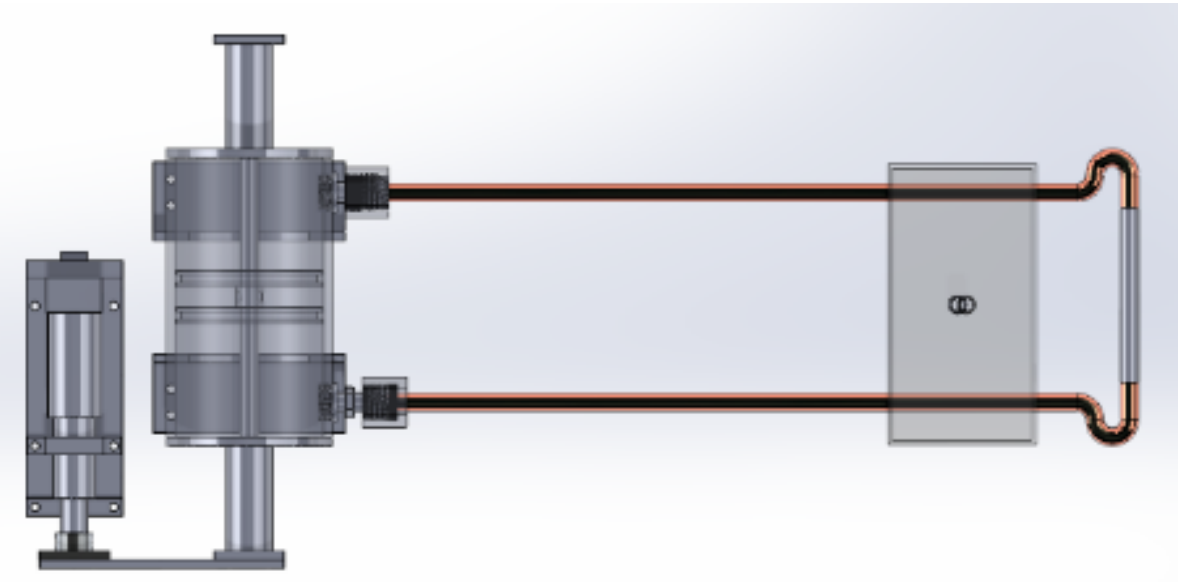

(a)

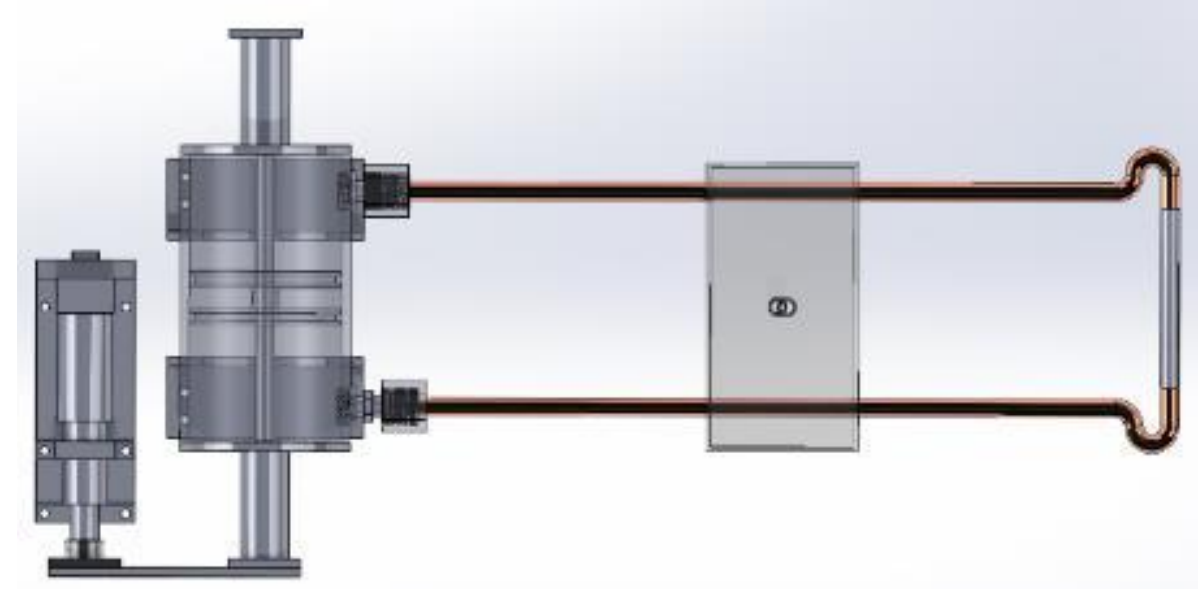

(b)

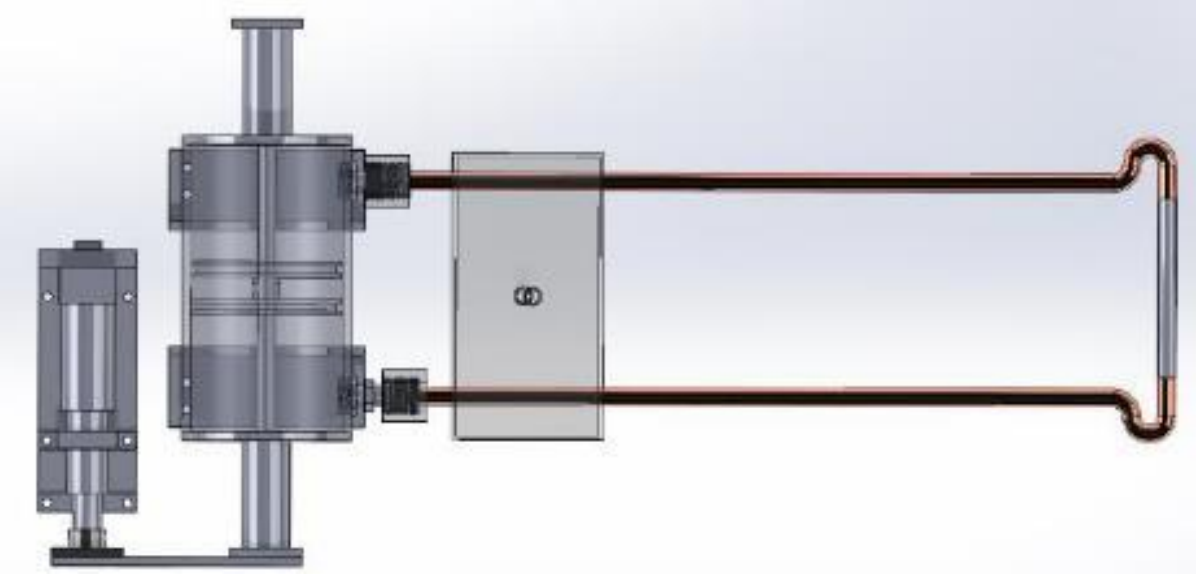

(c)

Figure 3. 9: Variations in RMDHL loop for single flow characterization showing different distances between heat source and condenser (a) configuration A (15 cm) (b) configuration $B(30 \mathrm{~cm})(\mathrm{c})$ configuration $C(60 \mathrm{~cm})$ 


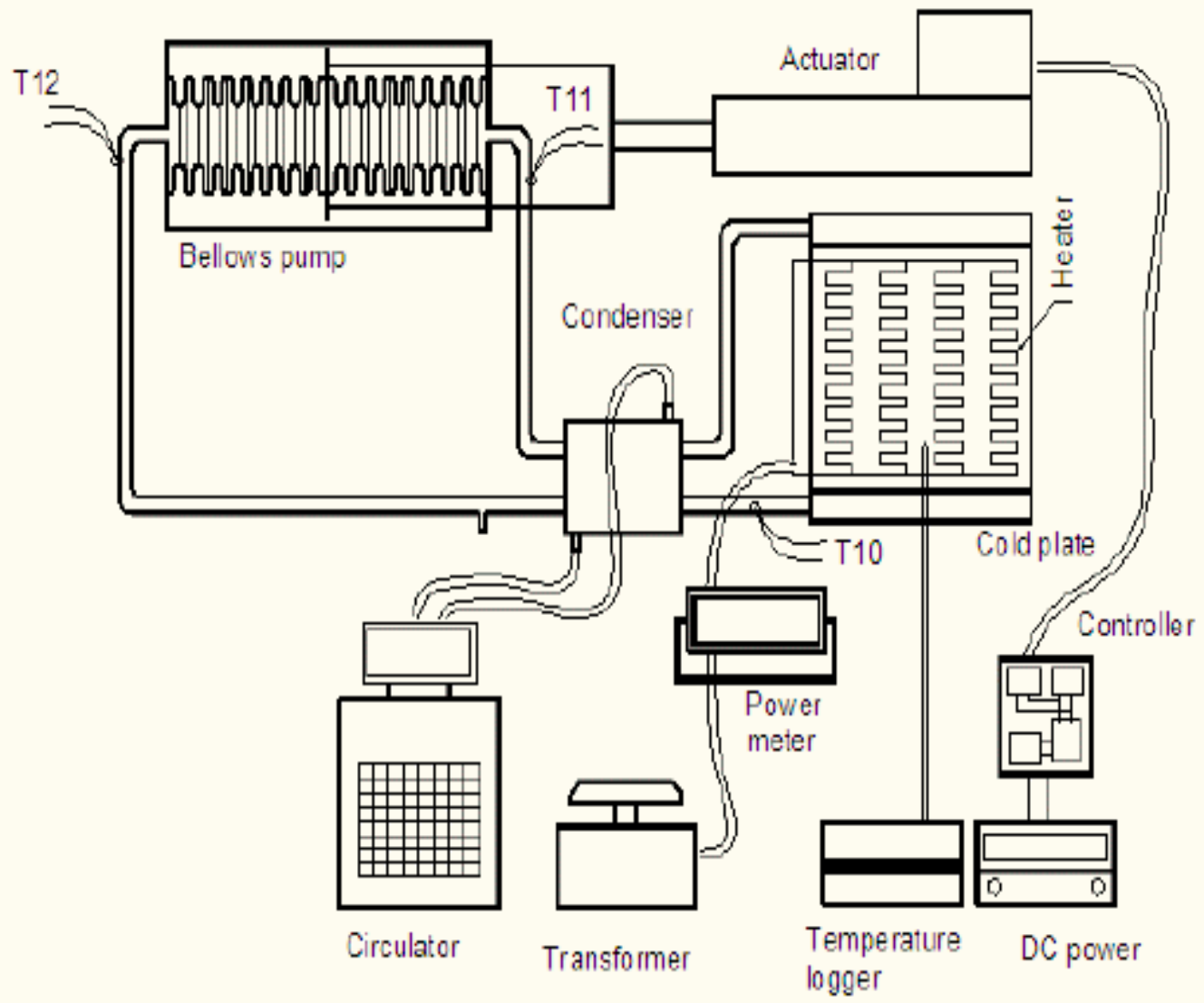

Figure 3. 10: Overall arrangement of Single Phase Experimental Setup $(\mathrm{Cao}, \mathrm{Xu}$, and Gao 2013) 
$7.32 \mathrm{~mm}$ with a wall thickness of $1 \mathrm{~mm}$ and a total length of $150 \mathrm{~cm}$. Thermocouples were placed at the evaporator to provide the temperature of the wall of the evaporator. Thermocouples were spaced $5 \mathrm{~cm}$ apart from the driver to the RMDHL driver.

The overall arrangement for the single-phase testing is shown in Fig 3.10. The sources of the experimental uncertainty were only the instruments themselves. The scanning thermocouple thermometer has an accuracy of $\pm 0.1 \%$ of reading $\pm 0.4{ }^{\circ} \mathrm{C}$. The power meter has an accuracy of $\pm(1 \%$ reading +5 digits $)$. So, the maximum uncertainty for the temperature and heating power measurement would be $1.0 \%$ and $2 \%$ respectively.

\subsection{Design and Construction of a Two-Phase Experimental Setup}

Preliminary two-phase studies involving the RMDHL by Cao et al., (2008) were conducted using the solenoid type RMDHL. The experimental results show that the heat loop worked very effectively and a heat flux as high as $300 \mathrm{~W} / \mathrm{cm}^{2}$ in the evaporator section could be handled. Cao et al., (2008) generated a few empirical performance data, and also derived a working criterion, which could provide a guidance for the design of a reciprocating-mechanism driven heat loop. However, these studies also show that the efficiency of the solenoid type RMDHL is limited by the strength of the magnetic field generated by the solenoid driver. With the development of the bellows-type RMDHL driver that provides a significantly higher driving torque than the solenoids driver, this study could significantly extend the performance of the 
RMDHL by developing a two-phase evaporator that will work with the bellows-type RMDHL.

The approach adopted in this study is to generate a robust data base of empirical performance data from the solenoid type RMDHL (using some of the data generated by Cao et al., (2008) and also generating additional empirical data) and then use this data base to develop numerical and analytical models that will be used to develop a new evaporator which can handle higher heat flux when coupled with the solenoids type RMDHL. In this study, the plan is to fabricate the new two-phase bellows-operated reciprocating loops and experimentally study the new loop using existing solenoids loop.

The schematic for experimental setup of this study based on the solenoid loop is illustrated in Fig. 3.11. A photograph of the apparatus is shown in Fig 3.12, which includes an electric heater for supplying heat to the evaporator of the heat loop, a power supply to the solenoid driver, a constant temperature circulator to maintain a constant coolant inlet temperature, a data acquisition system, and the heat loop to be studied. The portion of the heat loop from the evaporator section to the condenser section was placed in a horizontal position to eliminate the influence of gravity on the performance of the heat loop. 


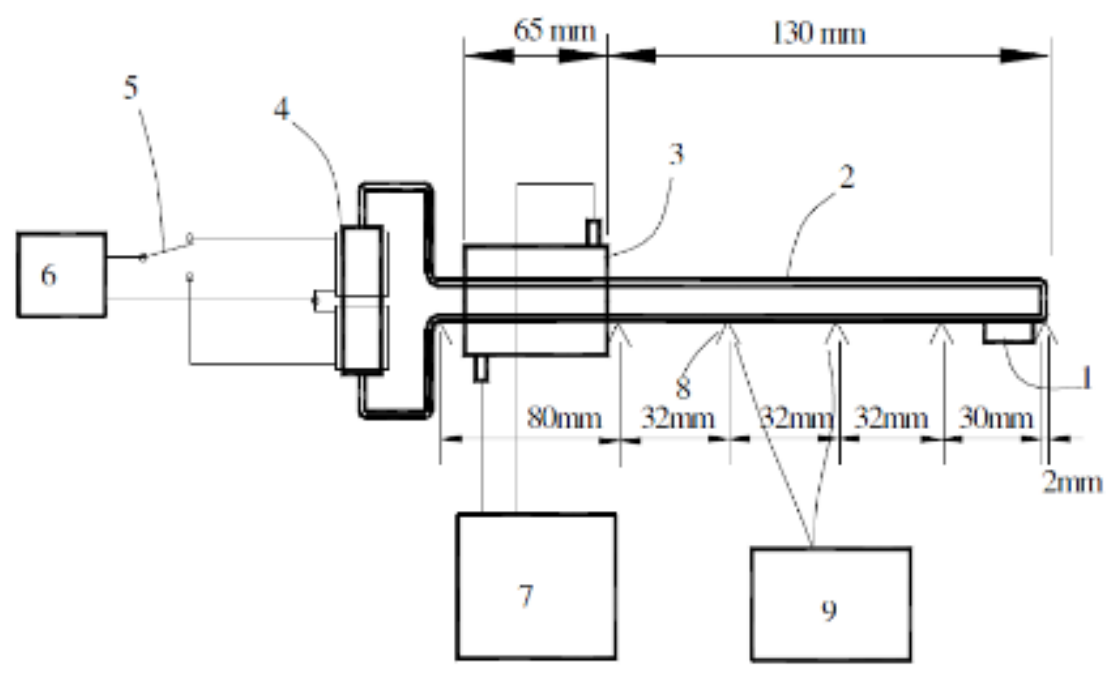

1-heater; 2 - heat loop; 3 - cooling jacket; 4 - solenoids; 5 - switch; 6 - power supply; 7 - constant temperature circulator; 8 -thermocouples; 9 - data acquisition system.

Figure 3. 11: Experimental setup for two-phase testing Bellow type

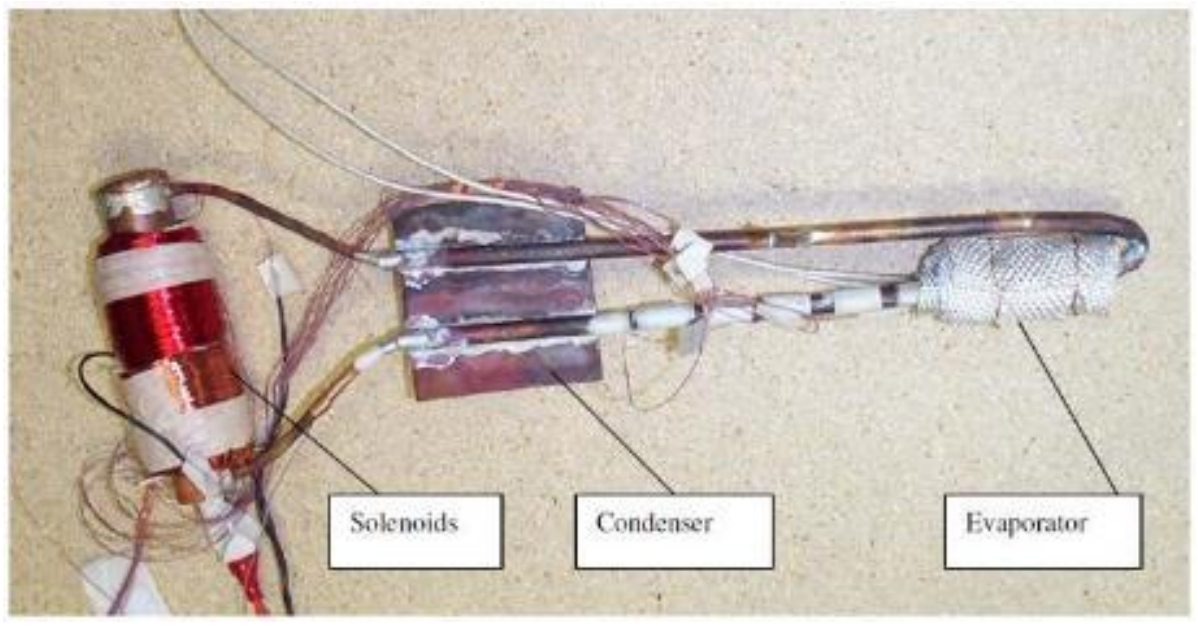

Figure 3. 12: A photograph of the fabricated reciprocating-mechanism driven heat loop 
The heat loop is made of copper tubing having an outer diameter of 0.25 " and a wall thickness of 0.035 ". The reservoir has an inner diameter of 0.75 " and a length of about $104 \mathrm{~mm}$. The heater is a small cartridge heater for providing a high heat flux heat input to the evaporator. The heater was welded onto the outside surface of the heat loop at the right end of the heat loop, as shown in Fig. 3. 12.

The contact surface area between the heater and the heat loop was approximately $0.56 \mathrm{~cm}^{2}$. This small contact surface area was deliberately set for providing a high heat flux to the evaporator. After the heater was welded to the heat loop, it was insulated from the ambient using a fiberglass tape to eliminate the heat loss from the heater to the ambient. Six thermocouples were placed on the outer surface of a branch of the heat loop with the first thermocouple being close to the heater to measure the axial temperature distribution. A liquid circulator is used to adjust and maintain a constant liquid inlet temperature at the cooling jacket so that the heat loop could operate at different working temperatures.

The distance between the right end of the heat loop and the cooling jacket and the length of the cooling jacket are also indicated in Fig. 3. 11. Similar to the single phase experimental setup, a control circuit board was used to control the reciprocating frequency of the solenoid driver. Water is used as working fluid for the study, and it occupied approximately $70 \%$ of the interior volume of the heat loop. The magnetic material of the reciprocating driver is carbon steel coated with copper to be compatible with water. 
The piston stroke in conjunction with the inner diameter of the reservoir is believed to be one of the most critical operational parameters because it would determine the oscillation amplitude of the liquid-vapor mixture and the rewetting capability of the liquid to reach from the condenser section to the evaporator section. The piston reciprocating frequency will have a favorable effect on the sweeping speed of the twophase mixture over the heated surface.

In addition to the parameters discussed above, a number of additional parameters were measured to generate useful experimental data. These parameter include the heat input, $q$, to the evaporator, the temperature difference between the heated wall surface and the working fluid, $\Delta T=T_{w}-T_{b}$, and the power input to the reciprocating driver, which may be related to the working fluid temperature under a two-phase condition.

The stroke of the driver was about $40 \mathrm{~mm}$, and the magnitude of the reciprocating frequency was $1 \mathrm{~Hz}$. The sources of the experimental uncertainty were only the instruments themselves, which have been described before.

\subsection{Single-Phase Heat Transfer Correlations}

In estimating the heat transfer between the evaporator and the condenser, it was assumed that flow in one pipe of the condenser is "parallel flow" in the forward cycle and "counterflow" in the reverse flow. Using the logarithmic mean temperature differences (LMTD), it turns out that the difference between the 'arithmetic difference' and 'logarithmic mean temperatures' is negligible. 


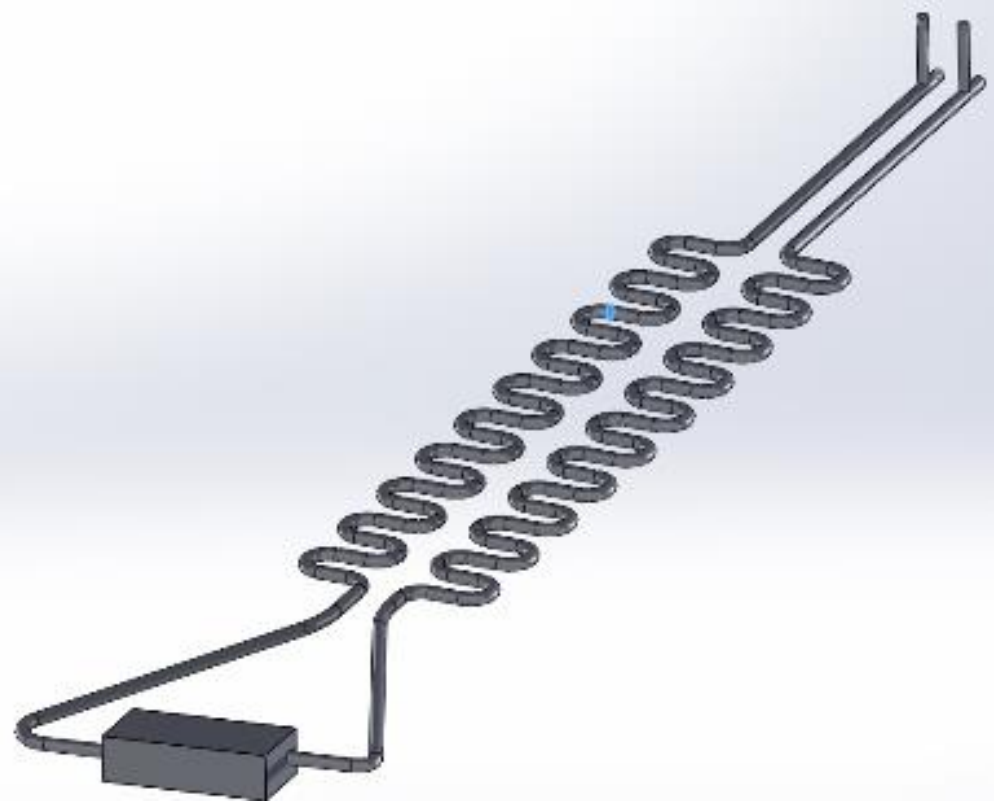

Figure 3. 13: Isometric view of the redesigned 2 phase loop 
Based on the variables present in Eq. 1 to Eq. 6, the frequency, amplitude, heat input and distance between the evaporator and condenser were varied during the experiment. Presented in Table 3.1 is the summary of input variations while the values for material properties were taken from Touloukian et al., (1970). The experimental data of heat transfer rate at different strokes and distances $\Delta x$ are summarized in Table 3.2 using the method of Taguchi. The experimental values of the effective thermal conductivity and convective heat transfer coefficient for the evaporator were determined by the following relations:

$$
\begin{aligned}
& k_{\text {eff }}=\frac{Q_{i n} \Delta x}{A_{c} \Delta T} \\
& h=\frac{Q_{\text {in }}}{A_{\text {catridge }} \Delta T_{f}} \\
& \frac{Q_{\text {in }}}{A_{\text {heater }}}=q=h_{e}\left(\overline{T_{\text {evap }}}-\overline{T_{f}}\right) ; \quad \overline{N u}=\frac{h_{e} D_{h}}{k_{\text {water }}}
\end{aligned}
$$

where $A_{c}$ is the pipe cross-sectional area, evaluated based on the inner diameter of the RMDHL loop tubing, $\Delta T$ is the difference in temperature between the evaporator and the condenser, while $\Delta x$ is the distance between the evaporator and the condenser. 
Table 3. 1: Summary of input variations for experimental investigation into $\mathrm{Nu}$ correlation

\begin{tabular}{|l|l|l|}
\hline Oscillating frequency & $1 \mathrm{~Hz}, 2 \mathrm{~Hz}$, \\
\hline Oscillation amplitudes & $3.81 \mathrm{~cm}, 7.62 \mathrm{~cm}, 15.24 \mathrm{~cm}$ \\
\hline Tube diameter & $0.3175 \mathrm{~cm}(\mathrm{OD}) \times 0.2362 \mathrm{~cm}$ (ID) \\
\hline Condenser dimension & \multicolumn{2}{|l|}{$10.16(\mathrm{~cm}) \times 15.24 \times 20.32$} \\
\hline \multirow{2}{*}{ Evaporator to condenser length } & $\mathrm{A}$ & $15 \mathrm{~cm}$ \\
\cline { 2 - 3 } & $\mathrm{B}$ & $30 \mathrm{~cm}$ \\
\cline { 2 - 3 } & $\mathrm{C}$ & $60 \mathrm{~cm}$ \\
\hline Heat input & $21.43 \mathrm{~W}, 42.86 \mathrm{~W}, 64.29 \mathrm{~W}, 85.71 \mathrm{~W}, 107.14 \mathrm{~W}$ \\
\hline
\end{tabular}

Table 3. 2: Experimental Grid for single phase characterization of RMDHL

\begin{tabular}{|c|c|c|c|c|}
\hline $\mathrm{S} / \mathrm{N}$ & $\omega$ & $\begin{array}{l}\text { Stroke } \\
(\mathrm{cm})\end{array}$ & $x(\mathrm{~cm})$ & $\mathrm{Q}(\mathrm{W})$ \\
\hline 1 & \multirow{13}{*}{1} & \multirow{8}{*}{3.81} & \multirow{5}{*}{15} & 11.90476 \\
\hline 2 & & & & 21.42857 \\
\hline 3 & & & & 35.71429 \\
\hline 4 & & & & 42.85714 \\
\hline 5 & & & & 57.14286 \\
\hline 6 & & & \multirow{3}{*}{30} & 11.90476 \\
\hline 7 & & & & 21.42857 \\
\hline 8 & & & & 35.71429 \\
\hline 9 & & \multirow{5}{*}{7.62} & \multirow{5}{*}{30} & 11.90476 \\
\hline 10 & & & & 21.42857 \\
\hline 11 & & & & 35.71429 \\
\hline 12 & & & & 42.85714 \\
\hline 13 & & & & 57.14286 \\
\hline
\end{tabular}




\begin{tabular}{|c|c|c|c|c|}
\hline 14 & & & & 64.28571 \\
\hline 15 & & & \multirow{7}{*}{60} & 11.90476 \\
\hline 16 & & & & 21.42857 \\
\hline 17 & & & & 35.71429 \\
\hline 18 & & & & 42.85714 \\
\hline 19 & & & & 57.14286 \\
\hline 20 & & & & 64.28571 \\
\hline 21 & & & & 71.42857 \\
\hline 22 & \multirow{24}{*}{2} & \multirow{16}{*}{3.81} & \multirow{7}{*}{15} & 11.90476 \\
\hline 23 & & & & 21.42857 \\
\hline 24 & & & & 35.71429 \\
\hline 25 & & & & 42.85714 \\
\hline 26 & & & & 57.14286 \\
\hline 27 & & & & 64.28571 \\
\hline 28 & & & & 85.71429 \\
\hline 29 & & & \multirow{4}{*}{30} & 11.90476 \\
\hline 30 & & & & 21.42857 \\
\hline 31 & & & & 35.71429 \\
\hline 32 & & & & 42.85714 \\
\hline 33 & & & \multirow{5}{*}{60} & 11.90476 \\
\hline 34 & & & & 21.42857 \\
\hline 35 & & & & 35.71429 \\
\hline 36 & & & & 42.85714 \\
\hline 37 & & & & 57.14286 \\
\hline 38 & & \multirow{8}{*}{7.62} & \multirow{8}{*}{60} & 11.90476 \\
\hline 39 & & & & 21.42857 \\
\hline 40 & & & & 35.71429 \\
\hline 41 & & & & 42.85714 \\
\hline 42 & & & & 57.14286 \\
\hline 43 & & & & 64.28571 \\
\hline 44 & & & & 85.71429 \\
\hline 45 & & & & 95.2381 \\
\hline
\end{tabular}


The following conclusions are drawn from the experimental data that are illustrated in Fig 3.14 to Fig. 3.17:

$k_{\text {eff }}$

1. is more than 400 times the thermal conductivity of copper and increases

with increasing amplitude, frequency and distance between the evaporator and condenser.

2. $h$ increases with increasing $\omega$ and amplitude of the driver, while the distance between the evaporator and the condenser has a low impact.

3. An increasing in $\omega$ significantly produces a drop in both the wall temperature and the bulk fluid temperature

4. The Prandtl number was between 1.96 and 5.43 .

5. Temperatures decreased as the amplitude of the RMDHL driver increased, indicating better heat transfer.

6. The ratio between the amplitude and the distance between the evaporator and condenser affects the heat transfer.

7. The increasing the distance between the evaporator increases the time for the flow to reach steady state. However, the impact on the local heat transfer is not pronounced after steady state is achieved. 


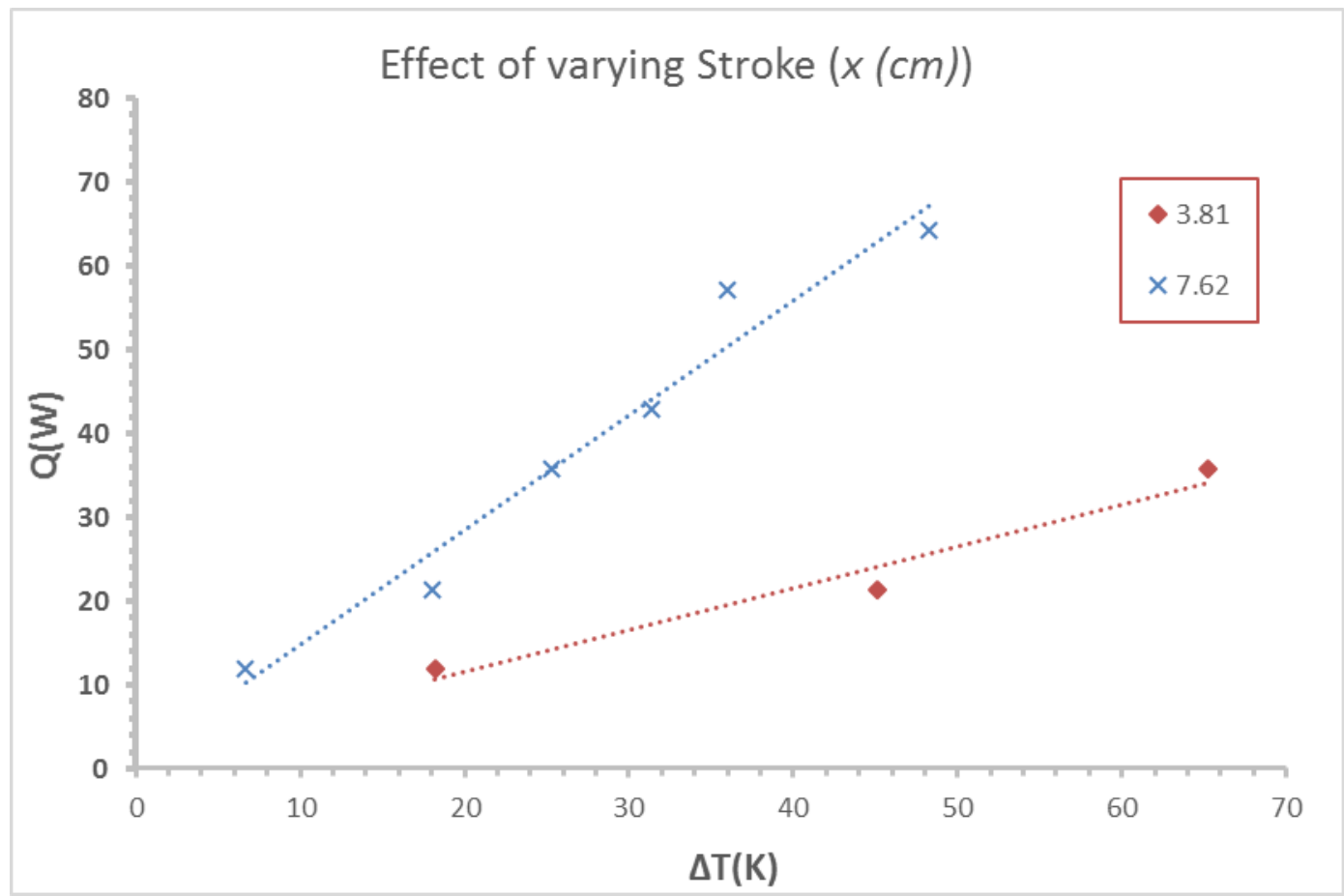

Figure 3. 14: $Q$ vs. $\Delta T$ at $\omega=1 \mathrm{rps}$, and $L_{E C}=30 \mathrm{~cm}$

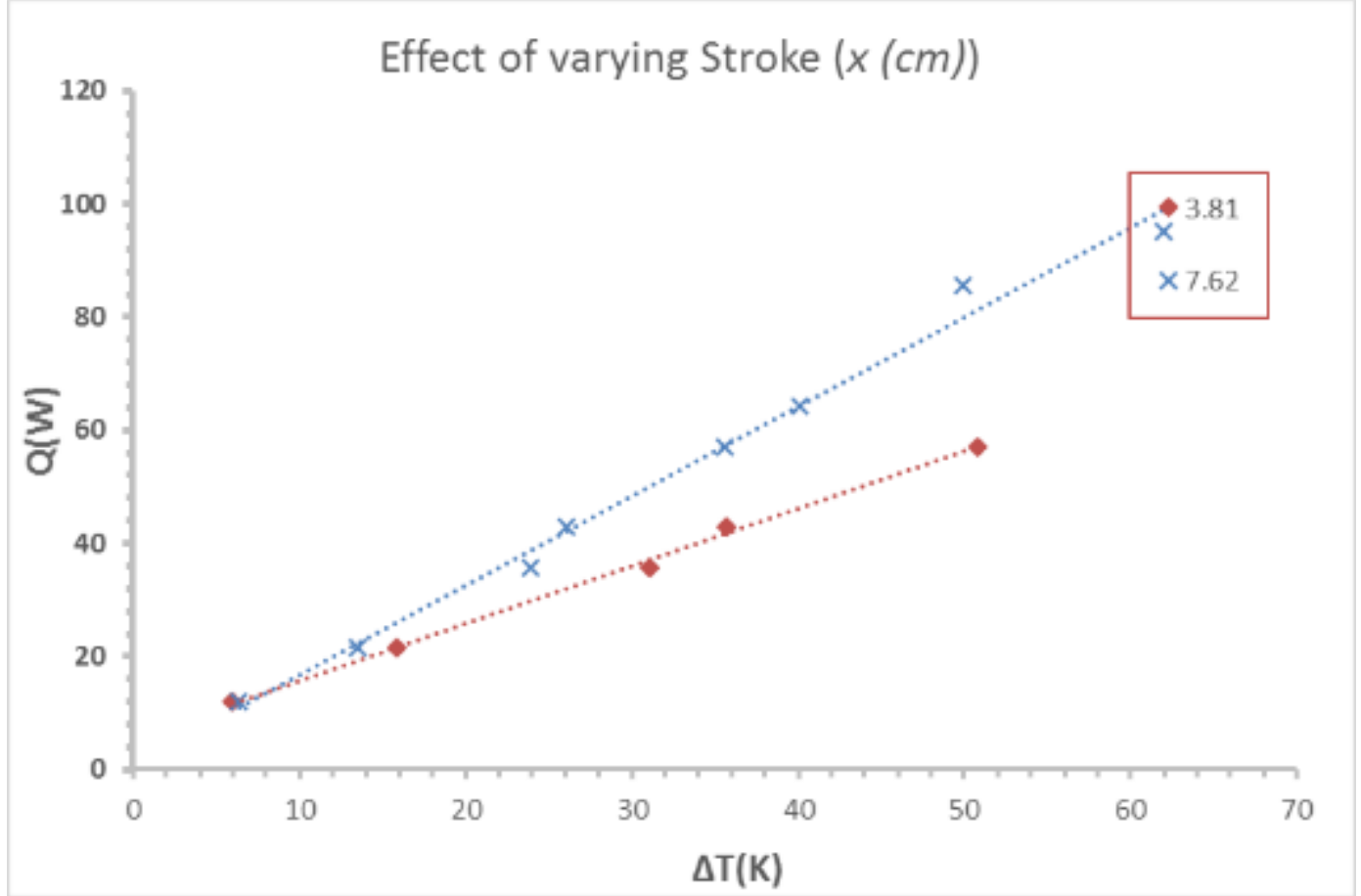


Figure 3. 15: $Q$ vs. $\Delta T$ at $\omega=2 \mathrm{rps}$, and $L_{E C}=60 \mathrm{~cm}$

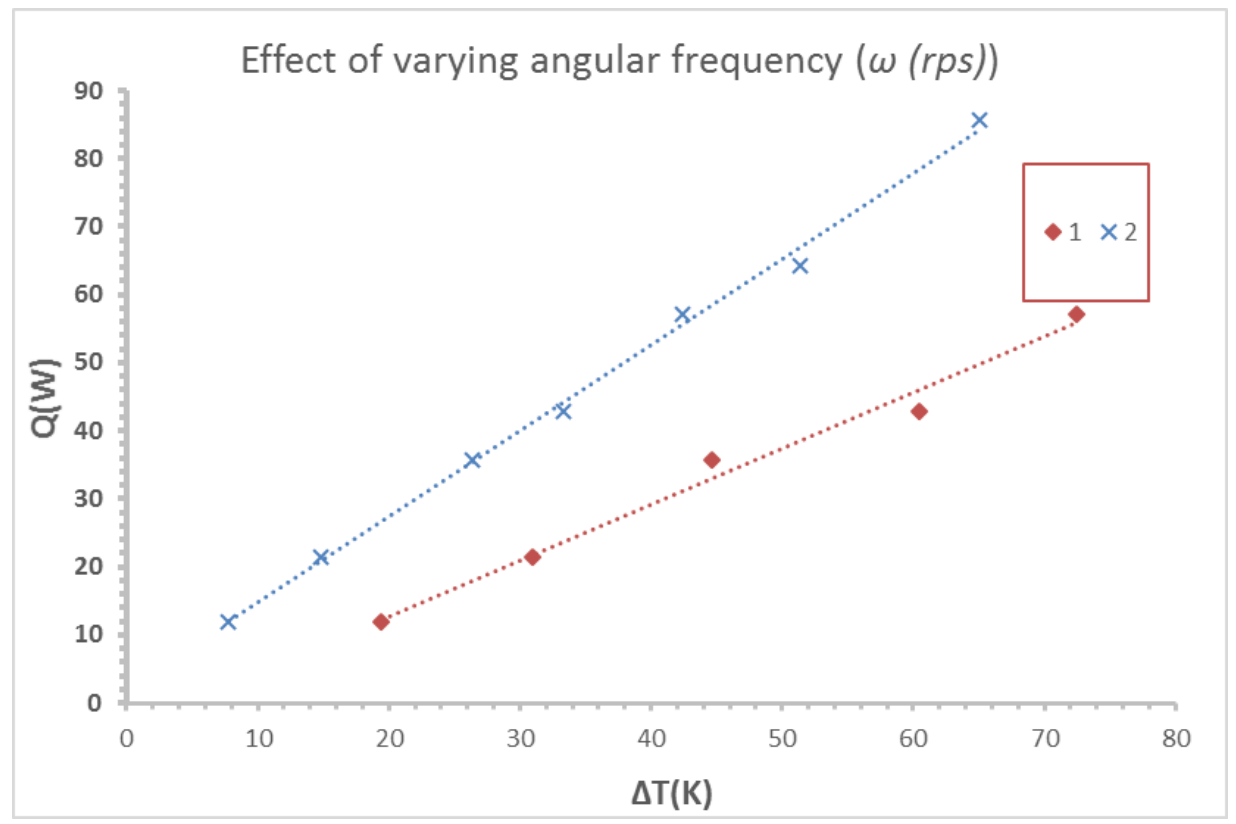

Figure 3. 16: $Q$ vs $\Delta T$ at $A=7.62$ and $L_{E C}=60 \mathrm{~cm}$

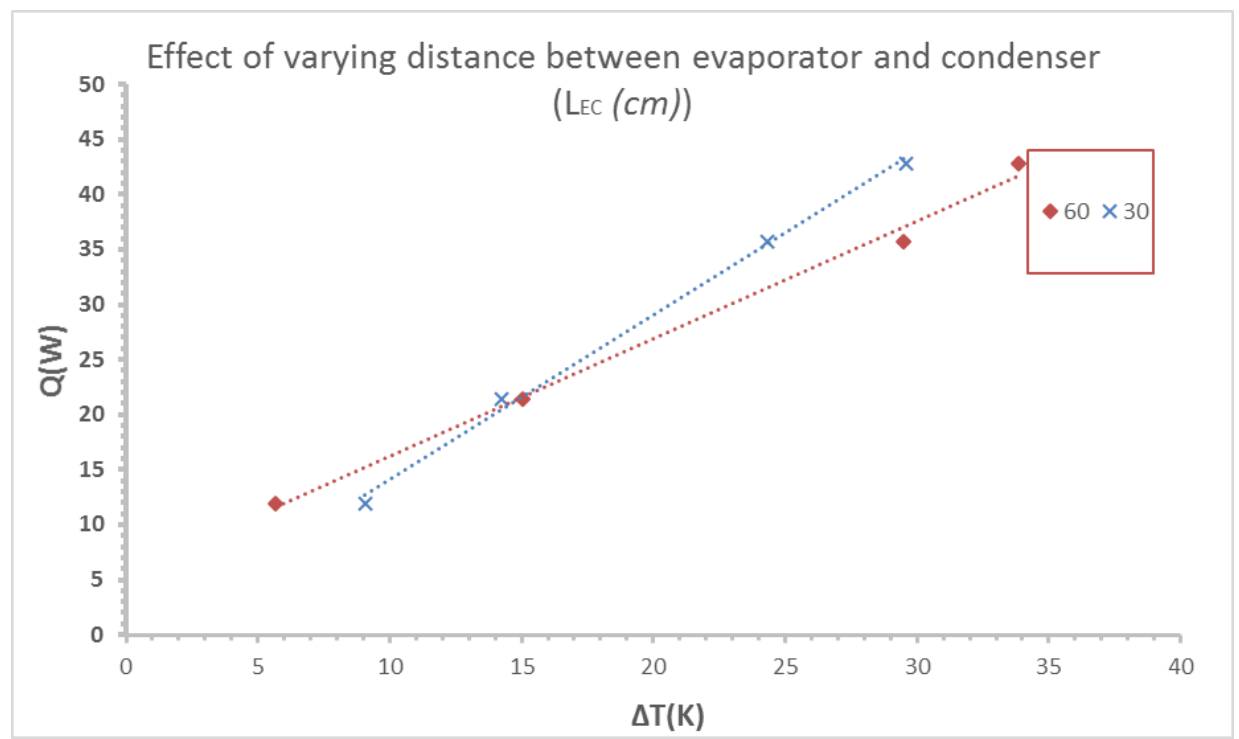

Figure 3. 17: $Q$ vs $\Delta \mathrm{T}$ at $\omega=2 \mathrm{rps}$ and Amplitude $=7.62 \mathrm{~cm}$ 
Data of $k_{\text {eff }}$ obtained from the experiment are shown in Table 3.3, and compared to the results from Shin's (Shin and Nishio 1998), as shown in Fig 3.18. As illustrated in Fig 3.18, the result of the experimental anlysis conformed with the prediction from the correlation by Shin (Shin \& Nishio, 1998), Eq.(12), to a very reasonable degree.

$$
k_{\text {eff }}=\left(\begin{array}{ll}
\rho C_{p} \frac{0.707}{\left(1+\frac{1}{\operatorname{Pr}}\right)\left(1+\frac{1}{\sqrt{\operatorname{Pr}}}\right)}\left(\frac{4 A^{2}}{D / 2}\right) \sqrt{\omega \kappa} ; & W_{o}>1 \\
\rho C_{p} \frac{1}{96}\left(1-\frac{13 \operatorname{Pr}^{2}+3}{2880}\right) \cdot\left(D / 2 \sqrt{\frac{\omega}{v}}\right)^{4}(D A)^{2}\left(\frac{\omega^{2}}{\kappa}\right) ; & W_{o} \leq 1
\end{array}\right.
$$

Nusselt numbers from 45 points in the experiments were calculated. From the values obtained, a non-linear correlation has been developed, and the expression for the evaporator is given as:

$$
\overline{N u}=3.27 W_{o}^{0.2}+\frac{0.0024\left(A / L_{e}\right) W_{o}^{2} \operatorname{Pr}}{1+0.017\left[\left(A / L_{e}\right) W_{o}^{2} \operatorname{Pr}\right]^{2 / 3}}
$$

where the working fluid is water, $D_{i}$ is the hydraulic diameter of the flow channel, $L$ is the heated length, and $W_{o}$ and $A$ are the Womersley number and the sinusoidal oscillation amplitude $(\mathrm{cm})$, respectively. The results obtained using Eq. (13) were compared with the experimental data of this study as well as those from (T. Zhao and Cheng 1995) and (Pamuk 2016), which are presented for a frequency of $1 \mathrm{~Hz}$ in Fig 3.19 and for a frequency of $2 \mathrm{~Hz}$ in Fig 3.20. This study observed that (Pamuk 2016) provides a consistent comparison, but underpredicts the value for the heat transfer coefficient, while the value of 
Table 3. 3: Calculation of Single phase thermal conductance based on experiment with $T_{c}=45^{\circ} \mathrm{C}$

\begin{tabular}{|c|c|c|c|c|c|c|c|c|}
\hline $\mathrm{q}$ & $\mathrm{T}_{\mathrm{w}}(\mathrm{C})$ & $\mathrm{T}_{\mathrm{f}}(\mathrm{C})$ & $\Delta \mathrm{T}_{\text {mean }}(\mathrm{C})$ & $\Delta \mathrm{T}_{\max }(\mathrm{C})$ & $\mathrm{q}(\mathrm{W})$ & Area $\left(\mathrm{m}^{2}\right)$ & $\mathrm{L}(\mathrm{m})$ & $\mathrm{k}_{\text {eff }}(\mathrm{W} / \mathrm{mK})$ \\
\hline 55.40 & 65.35 & 59.23 & 6.12 & 11.41 & $989,285.71$ & 0.0000633 & 0.206 & $17,860.90$ \\
\hline 79.10 & 65 & 59.4 & 5.6 & 10.5 & $1,412,500.00$ & 0.0000633 & 0.206 & $27,711.90$ \\
\hline 86.60 & 64.5 & 54 & 10.5 & 18.5 & $1,546,428.57$ & 0.0000633 & 0.206 & $17,219.69$ \\
\hline 107.40 & 66 & 54.92 & 11.08 & 19.7 & $1,917,857.14$ & 0.0000633 & 0.206 & $20,054.75$ \\
\hline 137.20 & 68 & 57.4 & 10.6 & 19.5 & $2,450,000.00$ & 0.0000633 & 0.206 & $25,882.05$ \\
\hline
\end{tabular}

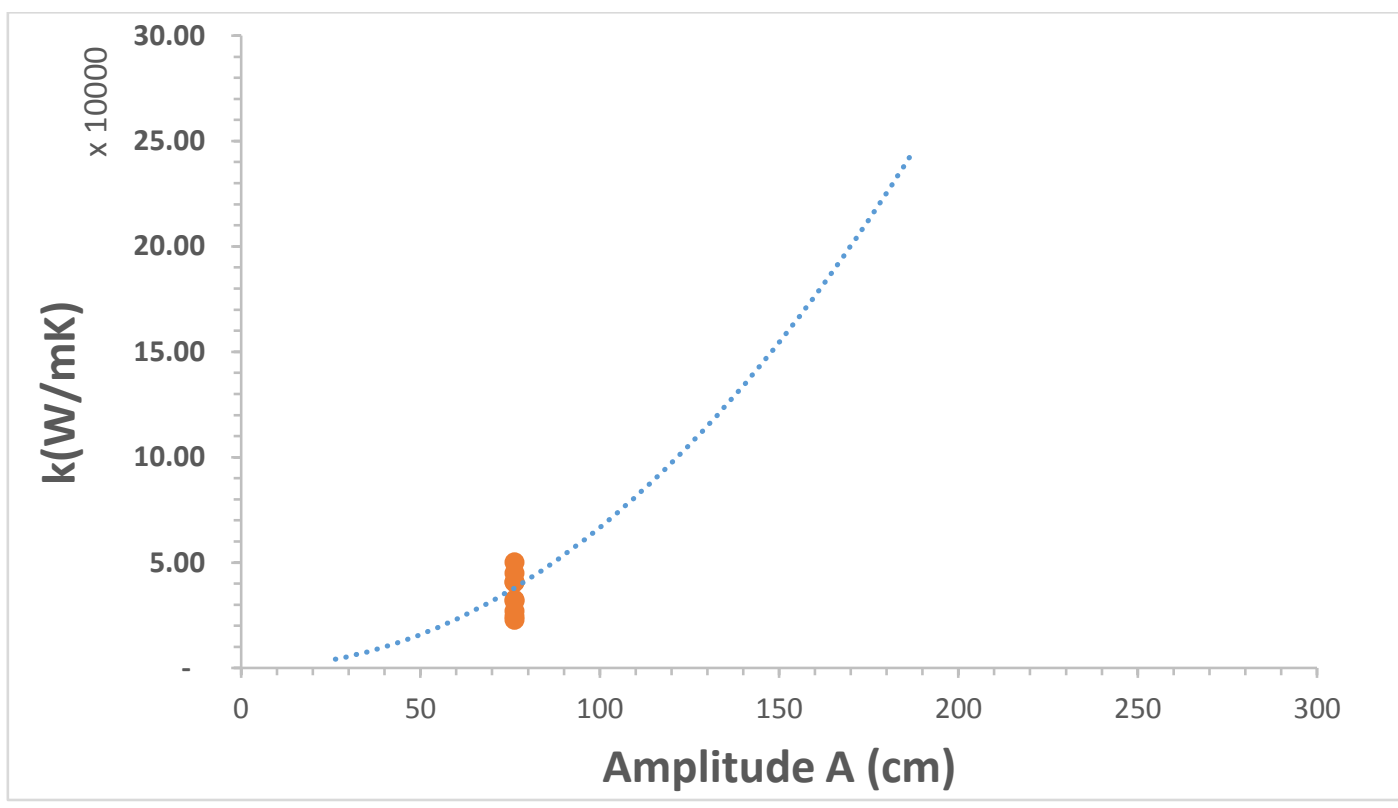

\footnotetext{
$k_{\text {eff }}$

Figure 3. 18: Comparison of experimental with correlations from Shin Shin \& Nishio (1998)
} 


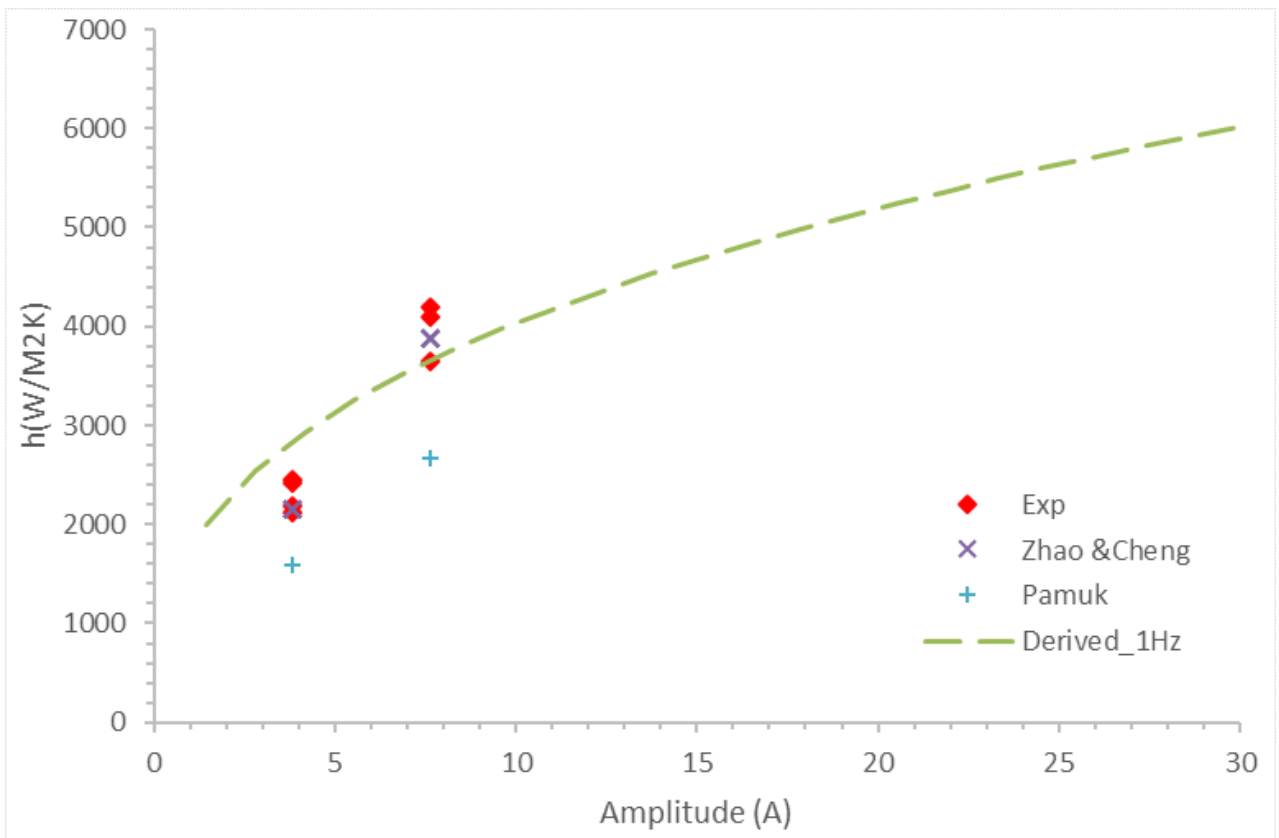

Figure 3. 19: Comparison of experimental heat transfer coefficient with various correlations at $\mathbf{f}=\mathbf{1 H z}$. 


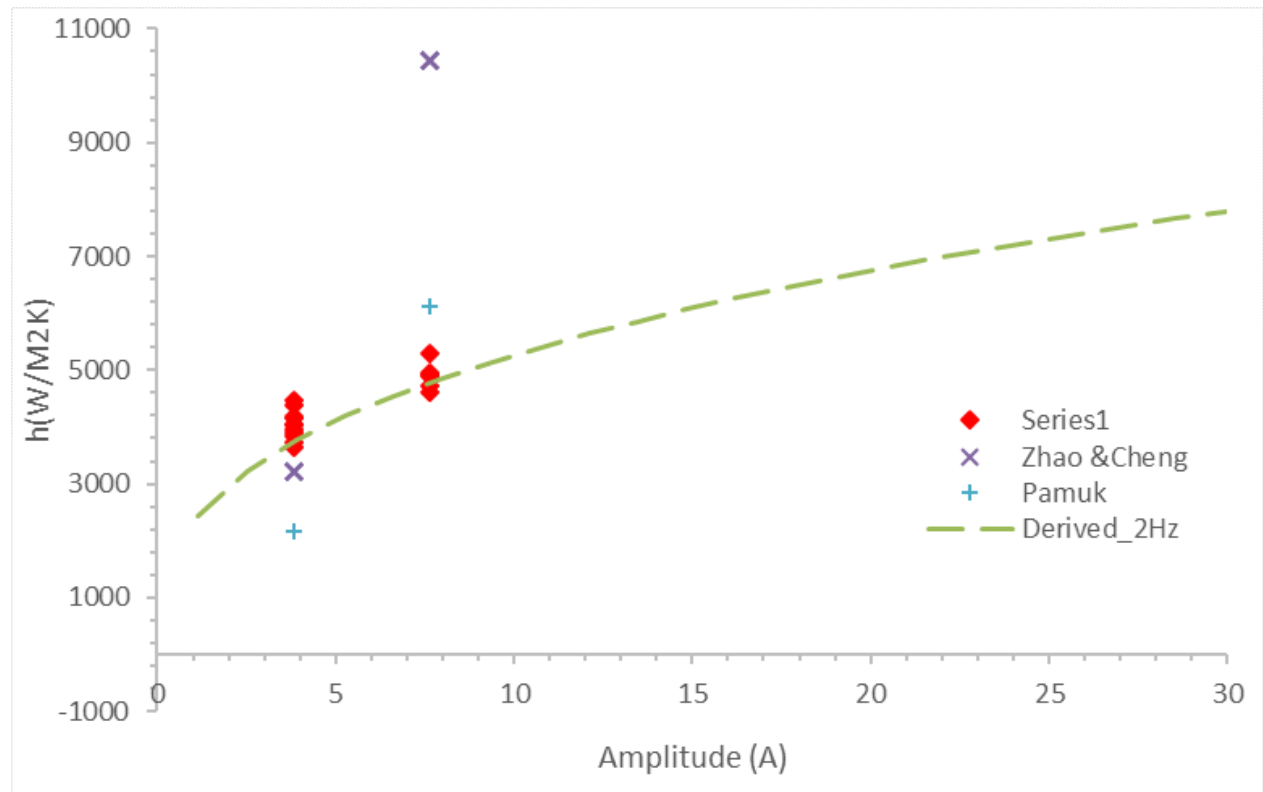

Figure 3. 20: Comparison of experimental heat transfer coefficient with various correlations at $\mathbf{f}=\mathbf{2 H z}$. 
(T. Zhao and Cheng 1995) was accurate at low oscillating frequent but deviate from the correlation with increasing speed. The correlation was then presented in a visually $k_{\text {eff }}$

representative format using MATLAB. and $\overline{\mathrm{Nu}}$ from the current study are presented in Fig. 3. 21 and Fig. 3.22, respectively. The idea is to develop simplified design tools that can be quickly interpreted for practical designs of heat transfer devices involving a reciprocating flow. The experiment shows that the single-phase RMDHL of this study could achieve an effective thermal conductance hundreds of times higher than the thermal conductance of copper $(400 \mathrm{~W} / \mathrm{m}-\mathrm{K})$. The result also shows that the correlation is a good approximation for the .

\subsection{Temperature Measurements of Single-Phase Heat Transfer Experiments}

Temperature distributions on the cold plate and in the working fluid for single-phase heat transfer from the current study followed the same trends as the work by Cao and Gao (2013). The results show that the bellows-type RMDHL is an effective thermal management device for applications involving large heat transfer rates and over a large surface area. The tests show that the bellows-type RMDHL has the potential to maintain a heat-generating surface at an exceedingly uniform temperature and the maximum temperature difference over the cold plate is directly proportional to the power of the actuator and the working frequency. The results of the tests also show that with a power input of about $5 \mathrm{~W}$ into the driver $550 \mathrm{~W}$ of heat can be managed at the evaporator. Detailed temperature distributions will be presented in the next section of numerical modeling when the temperature data are used to validate the numerical results. 


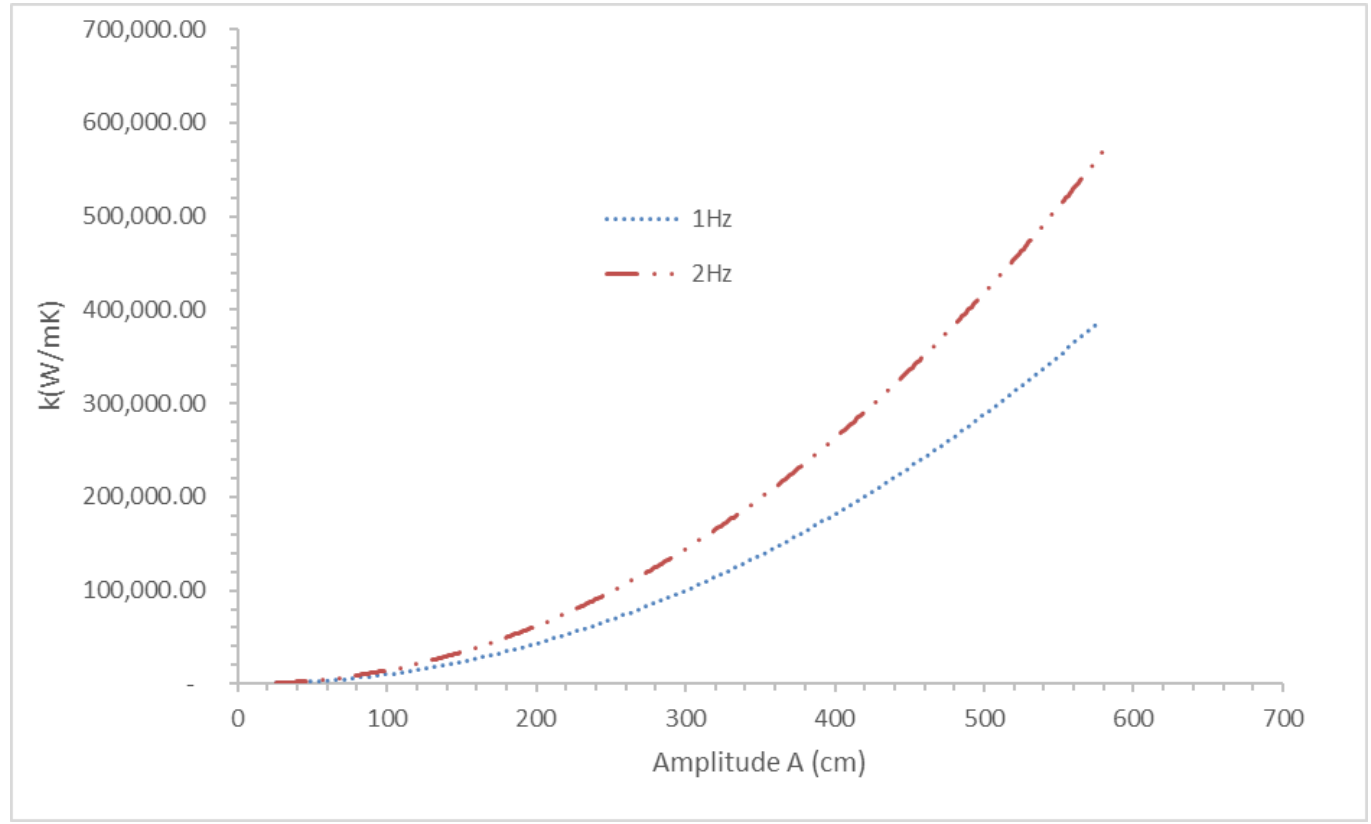

Figure 3. 21: Effective heat transfer coefficient against stroke for RMDHL 


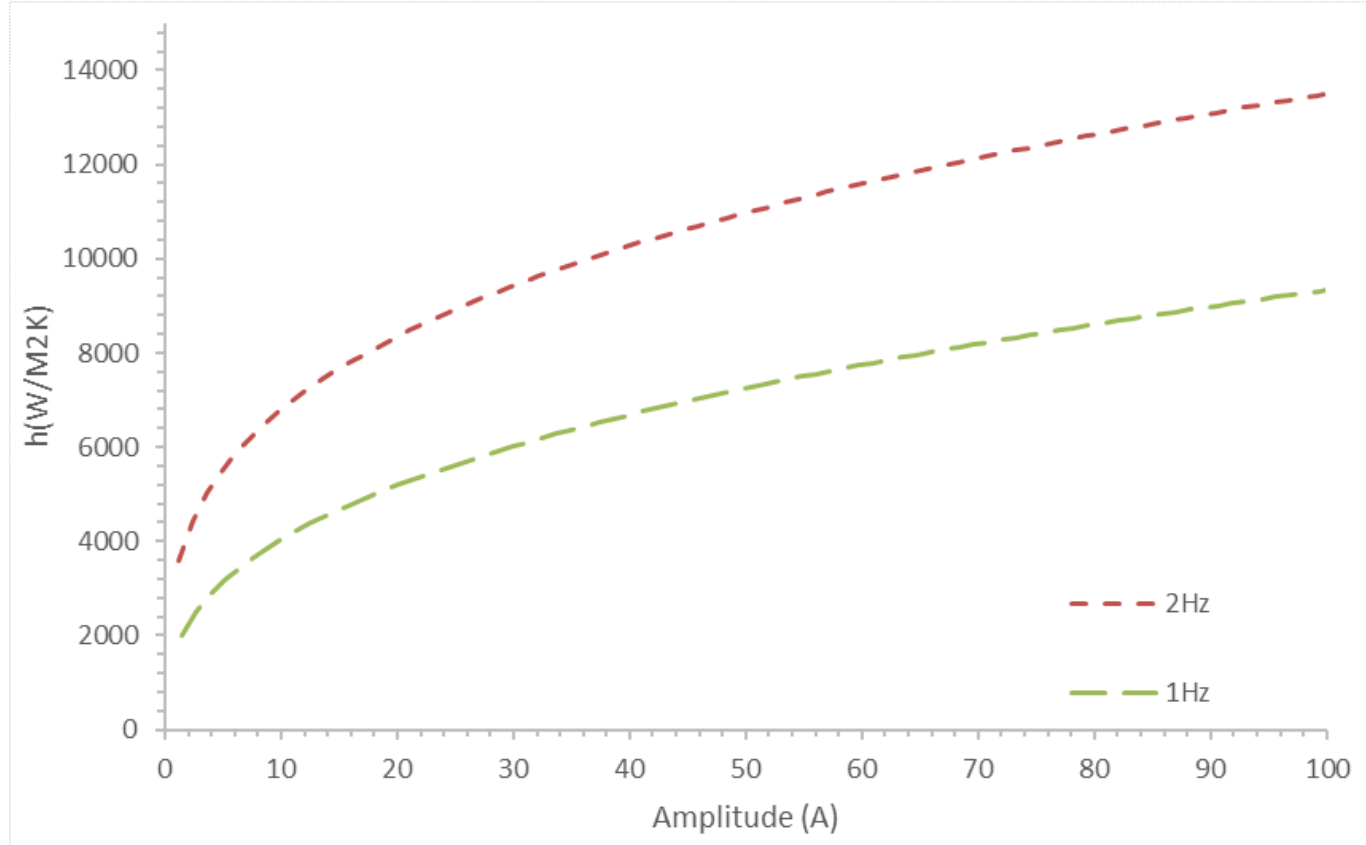

Figure 3. 22: Heat transfer coeffcient against stroke for RMDHL 


\section{CHAPTER FOUR: NUMERICAL STUDIES}

The task of developing a CFD model for reciprocating or oscillating flowin this studyis not a simple one. The problem is characterized by unsteady velocity profile due to the periodical change in flow direction, the coupled heating and cooling of the working fluid, and the needs to incorporate the effect of numerous fluid properties such as density, viscosity, thermal conductivity and specific heat. Then there is also the dependency of the model on the geometry and the flow boundary conditions (Bergman, Lavine, and Incropera 2012). An extensive review of literature revealed that the investogation into heat transfer in oscillating pipe flow has been mostly experimental or analytical studies focusing on pulsating pipe flow, Stirling engines and Vuilleumier heat pumps type systems, high Reynolds number open channel applications, and gravity-wave induced boundary layer in biological systems like arteries and airways (Nallasamy 1987; Markatos 1986; Gundogdu and Carpinlioglu 1999; Gündoğdu and Çarpınlığlu 1999; Özdinç Çarpinlioğlu and Yaşar Gündoğdu 2001; Ahn and Ibrahim 1991; M. Ibrahim and Hashim 1994; Su, Davidson, and Kulacki 2012; Ghafarian, Mohebbi-Kalhori, and Sadegi 2013; L. Wang and Lu 2004; Scotti and Piomelli 2001; M. Zhao, Cheng, and An 2010).

In the past the Stirling engine has been the basis for developing numerical

models for thermal management systems that achieve heat transfer using a 
reciprocating flow of the working fluid (Barreno et al. 2015). Similar to the Stirling engine, the principle of RMDHL operations for cooling applications may be theoretically described as heat transfer in a periodically reversing flow between two temperature reservoirs. In the operation of the Stirling Engine (illustrated in fig. 4.1 and Fig 4.2), the two reservoirs (the hot and cold reservoirs) can either be at constant heat flux or constant temperature. The hot and cold reservoirs are separated into in two distinct chambers which are connected by a regenerator. According to (Andersen, Carlsen, and Thomsen 2006), the Stirling engine is a closed cycle, regenerative engine in which work is expended to compress a cold gas, and the gas is then heated to further increased the pressure. The high temperature, high pressure gas is then expanded, and more work can be extracted than was needed to compress the cold gas. Finally, the gas is cooled before the next cycle begins with a new compression.

In the RMDHL, however, the compression and expansion are temperature induced, and the distinctions between the hot and the cold reservoirs is not as distinct. Also, the chambers are separated by an adiabatic region (as illustrated in Fig 4.3) which in reality is simply a connecting tubing. The implication is that the cooling and heating in the cycle of the working fluid must be numerically coupled and the numerical models for the Stirling engine are therefore not applicable for the RMDHL application. 


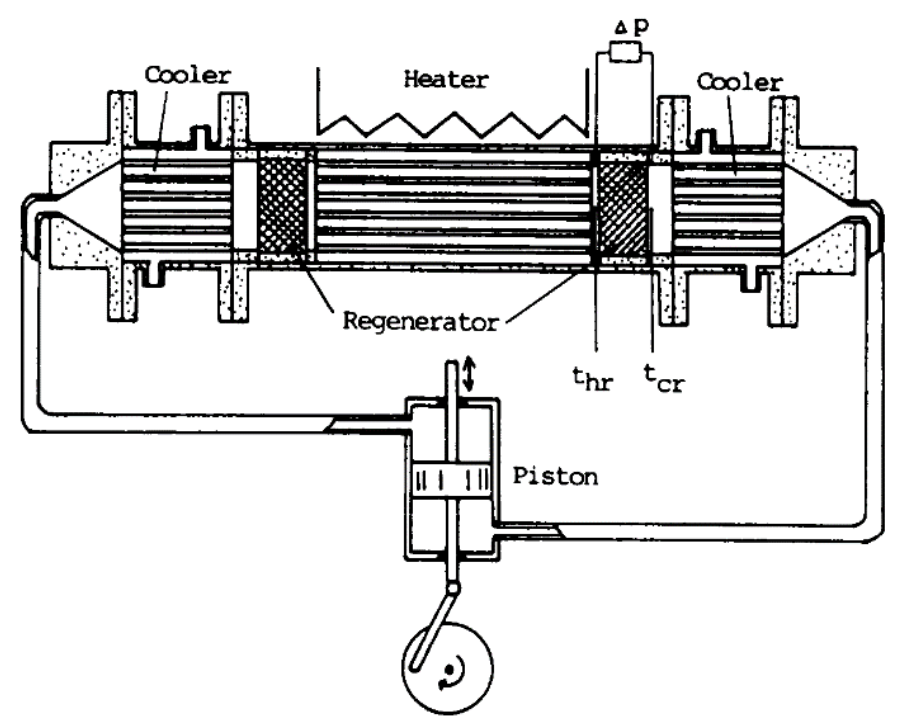

Figure 4. 1: Physical model of Sterling (Tanaka, Yamashita, and Chisaka 1990)

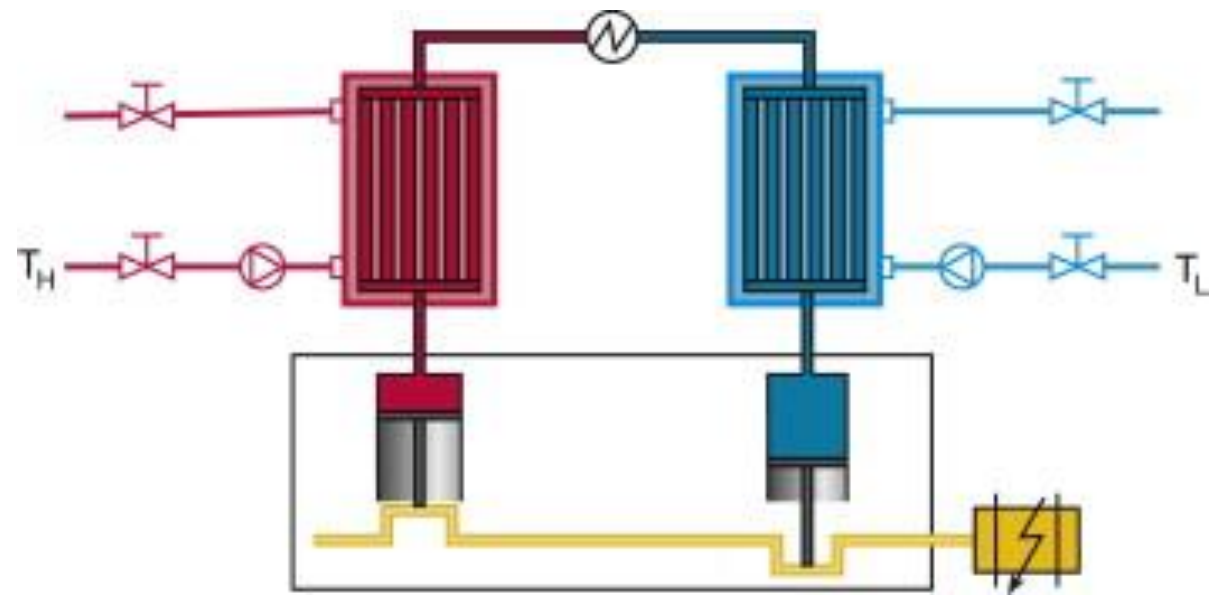

Figure 4. 2: Schematic diagram of the Alpha-type Stirling engine cooler (Kuosa et al. 2012) 


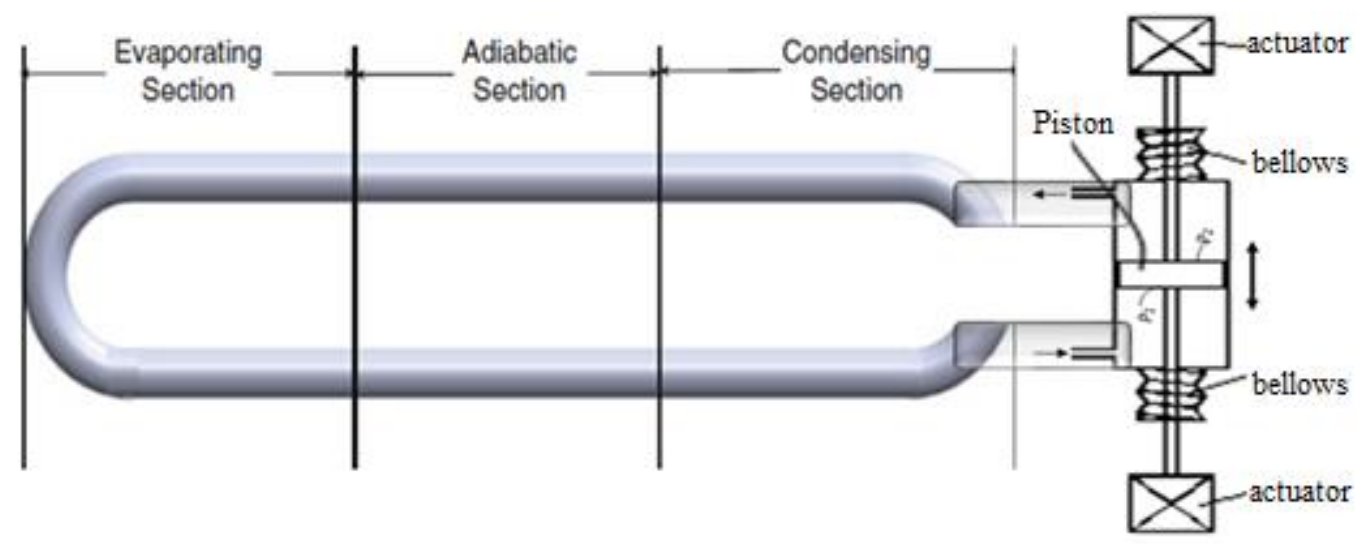

Figure 4. 3: Physical model of RMDHL 
It should also be mentioned that the Stirling engine and earlier oscillating flow cooling systems were driven by the circular motion of the driver. This circular motion translated into cosine or sine variation in the pressure form. Although the RMDHL can also be physically coupled with a rotary driver, the RMDHL of this study is the first oscillating flow device that incorporates a driver input controlled by an electrically driven actuator, and the waveform for the pressure oscillation can take any waveform programmed into the controller. Depending on the application, a square wave or a triangular wave can be programmed into the driver circuit. For the current study, the high temperature reservoir (the evaporator) is maintained at a constant heat flux while the cold reservoir is approximated to be at a constant temperature.

In developing a numerical model, the objectives of this study are as follows:

1. Develop a tool for analyzing the temperature profile across the cold plate to provide increased understanding of the cooling characteristics of the RMDHL

2. Define the velocity Profile of RMDHL and refine the accuracy of the heat transfer correlation

3. Investigate the transient temperature Profile on Cold Plate and the working fluid

4. Create a numerical tool for practical design and application of the RMDHL

5. Develop a two-phase numerical model for conjugate heat transfer involving reciprocating flow 
The initial portion of the current study has developed a single- phase, laminar oscillatory flow in the RMDHL to provide an estimate of the mean velocity and the velocity profile. The follow-up study investigated the most suitable turbulent model to predict the flow in the RMDHL more appropriately. Subsequent studies integrated the RPI boiling model into the turbulent oscillatory pipe flow developed earlier. Finally, this study analyzed vapor generation, transport and condensation within the RMDHL and validated the developed numerical model with specific characteristics of reciprocatory flow heat transfer. The general external boundary conditions for both single-phase and two-phase flows of this study are presented in Fig 4.4.

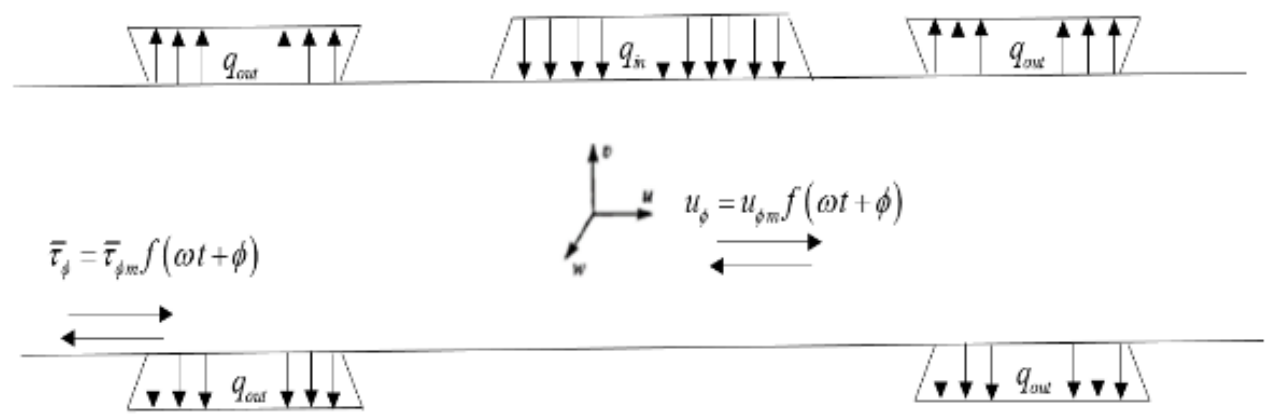

Figure 4. 4: External boundary conditions for this study

\subsection{Governing Equations for Single-Phase Flow}

The formulation in the present study is based on the two and three-dimensional conservation equations of mass, momentum, and energy under the conditions of incompressible fluid, negligible thermal dissipation and pressure work, constant fluid 
properties, and the Boussinesq approximation, along with appropriate boundary conditions, as shown in Fig. 4.4 All of the surfaces of the heat source exposed to the surroundings are assumed to be adiabatic except the interior walls of evaporator where a constant heat flux simulates the heat generation from the heat source. Based on above assumptions, the governing equations for fluid and energy transport are as follows:

$$
\begin{aligned}
& \nabla \cdot \vec{V}=0 \\
& \frac{\rho \partial(\vec{V})}{\partial t}+\rho(\vec{V} . \nabla \vec{V})=-\nabla p+\mu \nabla^{2} \vec{V} .
\end{aligned}
$$

Energy in fluid flow

$$
\rho c_{p} \frac{\rho D(T)}{D t}=-k_{s} \nabla^{2} T
$$

Other single-phase turbulent flow equations, are presented in section 4.3.

\subsection{Two-phase Numerical Models For RMDHL}

The boiling models are developed in the context of the Eulerian multiphase model. The multiphase flows are governed by the conservation equations for phase continuity, momentum, and energy. Given the maximum heat flux of about $300 \mathrm{~W} / \mathrm{cm}^{2}$ prescribed in the experiment, it is expected that the wall temperature is high enough to cause boiling to occur at the wall. Initial experimental results (Cao and Gao 2008) indicated that the bulk volume averaged liquid temperature is less than the saturation value. In this case, the energy is transferred directly from the wall to the liquid. Part of this energy will cause the temperature of the fluid to rise, and the other part will generate vapor. Interphase heat transfer will also cause the average liquid 


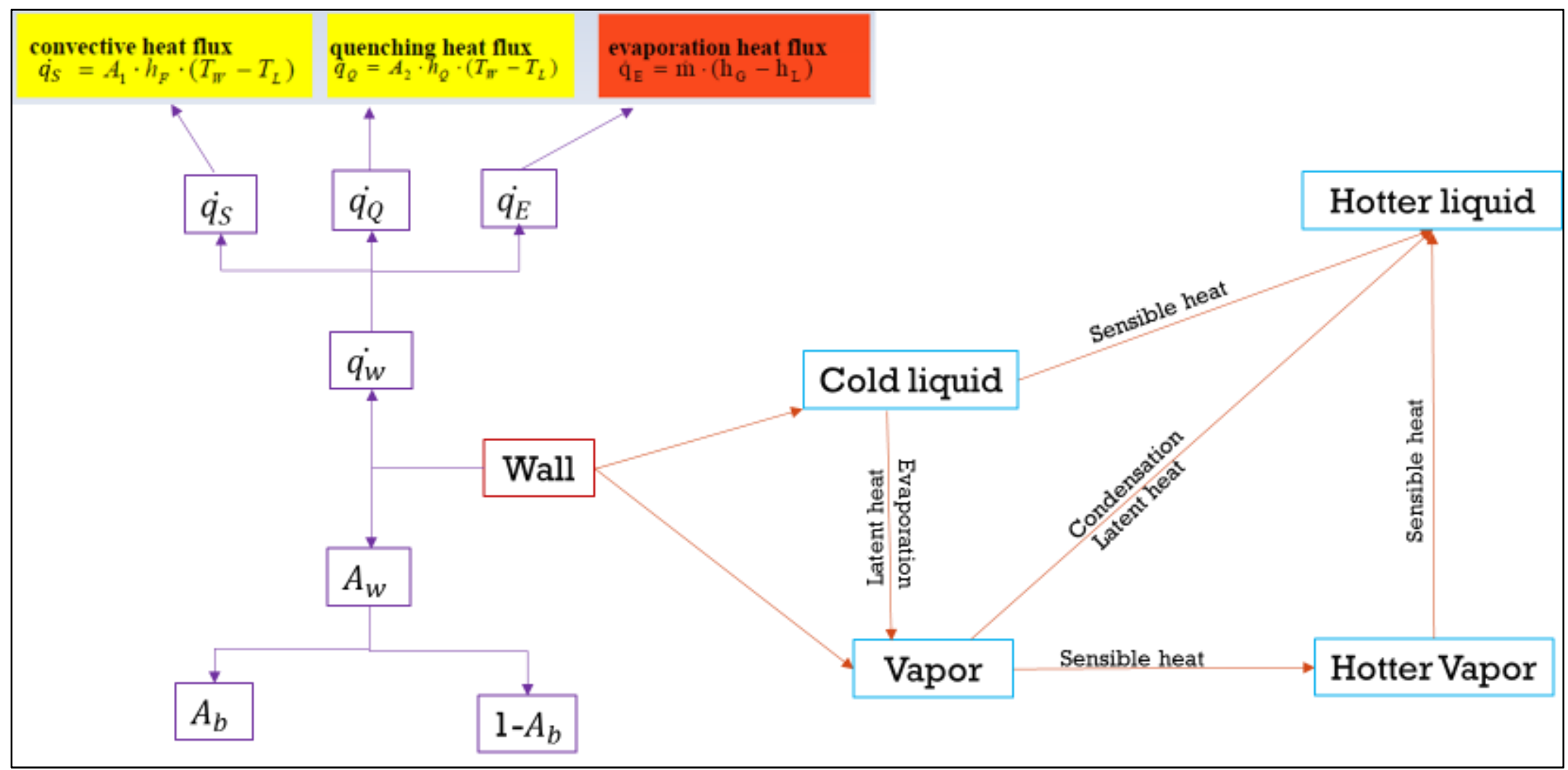

Figure 4. 5: Schematic of the distribution of wall heat flux in the two phase model 
temperature to increase; however, the vapor will condense at nearly saturated temperature. Additionally, some of the energy may be transferred directly from the wall to the vapor while traveling from the evaporator to the condenser. Fig. 4.5 is an illustration of the expected energy distribution of the wall heat flux, the interactions between the energy in each phase and the wall distribution due to bubble formation in the two-phase model captured in the current study. Each of the terms in Fig. 4. 5 is defined in susequent sections of this chapter.

The mechanisms defined in the previous paragraph is synonymous with the term "subcooled boiling". This term is used to describe the physical situation where the wall temperature is high enough to cause boiling to occur at the wall even though the bulk volume averaged liquid temperature is less than the saturation value. Subcooled boiling is the foundations of the so-called Rensselaer Polytechnic Institute (RPI) models (Kurul and Podowski 1990, 1991). Basically, the framework of Eulerian model is applied to the vapor-liquid two-phase flow and a wall heat flux partitioning model proposed by Rensselaer Polytechnic Institute (RPI) is used to describe the subcooled boiling process at heated wall surface (Gu et al. 2017). In the current study, the RPI model was modified, respectively, for wall heat flux/flux partitioning and evaporation/condensation in the bulk flow.

According to the basic RPI model, the total heat flux from the wall to the liquid is partitioned into three components, namely the convective heat flux, the quenching heat flux, and the evaporative heat flux: 


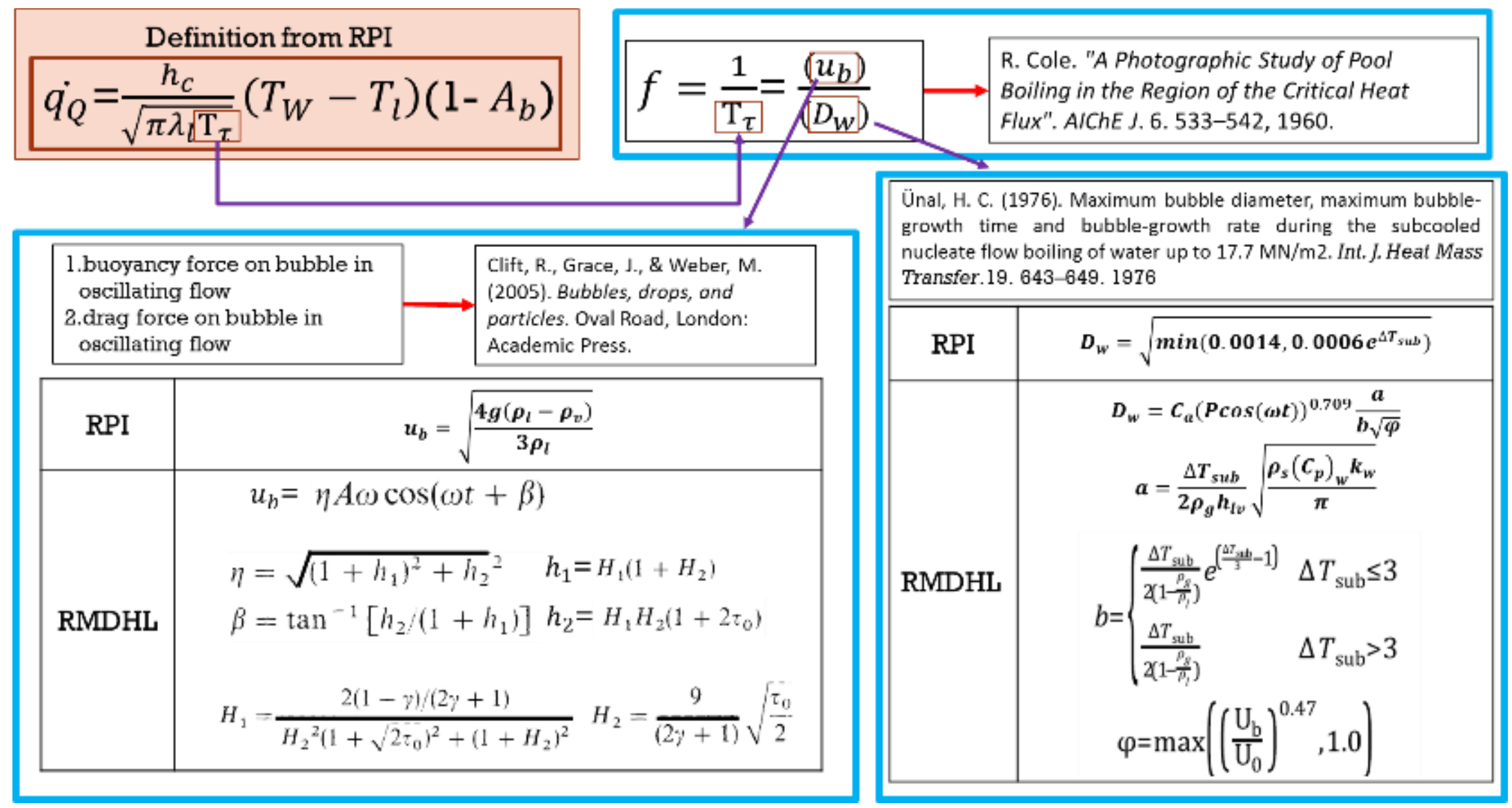

Figure 4. 6: Illustration of how how the RPI model was extended for two phase analysis of reciprocating two phase flow using quenching heat flux $\left(q_{Q}\right)$ and case study 


$$
q_{W}=\dot{q_{C}}+\dot{q_{Q}}+\dot{q_{E}}
$$

In order to describe the procedure for modifying the RPI model adopted in the current study, Fig 4.6 provides an illustration of the steps for redefining the quenching heat flux $\left(\dot{q}_{Q}\right) \cdot \dot{q_{Q}}$ is defined from RPI as:

$$
\dot{q_{Q}}=\frac{h_{c}}{\sqrt{\pi \lambda_{l} T_{\tau}}}\left(T_{W}-T_{l}\right)\left(1-A_{b}\right)
$$

$T_{\tau}$ and $A_{b}$ are the phase dependent terms in the equation. $T_{\tau}$ is defined by Cole (1960) as:

$$
f=\frac{1}{T_{\tau}}=\frac{\left(u_{b}\right)}{\left(D_{w}\right)}
$$

The definition of $u_{b}$ and the $D_{w}$ defined in the RPI are then substituted with a suitable definition for oscillating flow. The substitute correlation is selected based on wide acceptability (based on literature review) or selected based on their compatibility with ANSYS. In this particular case $u_{b}$ is defined by Clift, Grace, and Weber (2005) and $D_{w}$ is defined by Ünal (1976) as shown in Fig 4.6. In general, for the two-phase model, each phase satisfies the conservation equations of mass, momentum, and energy. The focus of the numerical model is vapor generation, transport and condensation within the RMDHL, and validation of the developed numerical model. 


\subsubsection{Continuity Equation}

The phases are coupled at the interface by the balance relations for mass, momentum, and energy. The interphase interactions are modeled using the particle model that assumes a continuous-phase fluid which contains particles of a dispersedphase fluid or solid. In this model, the approximated particle diameter $d_{b}$, and the volume phase fraction $\alpha$ is used to estimate the interphase contact area. Using the mixture theory approach, the volume of phase $q, V_{q}$, is defined by;

$$
V_{q}=\int_{V} \alpha_{q} d V
$$

Two-phase are expected in this problem, as a result:

$$
\sum_{q=1}^{2} \alpha_{q}=\alpha_{l}+\alpha_{v}=1
$$

The continuity equation for phase $\mathrm{q}$ is

$$
\frac{\partial}{\partial t}\left(\alpha_{q} \rho_{q}\right)+\nabla \cdot\left(\alpha_{q} \rho_{q} \vec{v}_{q}\right)=\sum_{p=1}^{n}\left(\dot{m}_{p q}-\dot{m}_{q p}\right)
$$

where $\vec{v}_{q}$ is the velocity of phase $q$ and $\dot{m}_{p q}$ characterizes the mass transfer from the $p^{t h}$ to the $q^{\text {th }}$ phase;

\subsubsection{Mass Transfer from the Wall to Vapor}

Mass Transfer from liquid to vapor which occurs at the cold wall is derived from the evaporation heat flux and was modeled as follows: 


$$
\dot{m}_{E}=\frac{\dot{q}_{E}}{h_{f v}+C_{p_{v}}\left(T_{s a t}-T_{l}\right)}
$$

where $C_{p_{v}}$ is the heat capacity of the coolant at constant pressure and $\dot{q}_{E}$ defined in the next section is the evaporative heat flux.

\subsubsection{Energy Equation}

For the wall boiling flow, a complete description of the energy transfer will need to account for the total energy transferred directly from the wall to the cooling fluid and energy transfer within the cooling fluid(s). This description is achieved using the RPI model. According to the RPI model (Kurul and Podowski 1991, 1990; Podowski, Alajbegovic, and Kurul 1997), the total heat flux from the wall to the liquid ( $\left.\dot{q}_{W}\right)$ is modeled as:

$$
\dot{q}_{W}=\dot{q}_{C}+\dot{q}_{Q}+\dot{q}_{E}
$$

where $\dot{q}_{C}$ is the convective heat flux, ${ }_{Q}$ is the quenching heat flux, and $\dot{q}_{E}$ is the evaporative heat flux.

$$
\dot{q}_{C}=h_{c}\left(T_{w}-T_{l}\right)\left(1-A_{b}\right)
$$

where $h_{c}$ is the single-phase heat transfer coefficient, $T_{w}$ and $T_{l}$ are the wall and liquid temperatures respectively, $A_{b}$ is the area of the heated wall surface covered by 
nucleating bubbles, making $\left(1-A_{b}\right)$ the area of the heated wall which is covered by the liquid.

$$
\dot{q}_{Q}=\frac{2 k_{l}}{\sqrt{\pi \lambda_{l} \tau}}\left(T_{w}-T_{l}\right)
$$

where the conductivity of the liquid phase is $k_{l}, \tau$ is the periodic time, and $\lambda_{l}$ is the diffusivity of the liquid phase.

$$
\dot{q}_{E}=f V_{d} N_{w} \rho_{V} h_{f v}
$$

where $V_{d}$ is the volume of the bubble based on the bubble departure diameter, ${ }^{w}$ is the active nucleate site density, $\rho_{V}$ is the vapor density, ${ }_{f v}$ and is the latent heat of evaporation, and $f$ is the bubble departure frequency. The definition of the other parameters associated with these equations is provided in (Del Valle and Kenning 1985; Kocamustafaogullari and Ishii 1983; Cole 1960; Park, Kim, and Miyata 1999).

The energy equation for each phase is:

$$
\begin{aligned}
& \frac{\partial}{\partial t}\left(\alpha_{q} \rho_{q} h_{q}\right)+\nabla \cdot\left(\alpha_{q} \rho_{q} \vec{u}_{q} h_{q}\right)=\alpha_{q} \frac{\partial p}{\partial t}+\bar{\tau}_{q}: \nabla \vec{u}_{q}-\nabla \vec{q}_{q} \\
& +S_{q}+\left(Q_{p q}+\dot{m}_{p q} h_{p q}-\dot{m}_{q p} h_{q p}\right)
\end{aligned}
$$

where $h_{q}$ is the specific enthalpy of the $q^{\text {th }}$ phase, $\vec{q}_{q}$ is the heat flux, and $S_{q}$ is a source term that includes sources of enthalpy (in this case 0 ) 


\subsubsection{The Momentum Equation}

The momentum balance for phase yields

$$
\begin{aligned}
& \frac{\partial}{\partial t}\left(\alpha_{q} \rho_{q} \vec{v}_{q}\right)+\nabla \cdot\left(\alpha_{q} \rho_{q} \vec{v}_{q} \vec{v}_{q}\right)=\alpha_{q} \nabla p+\nabla \cdot \bar{\tau}_{q}+\left(\alpha_{q} \rho_{q} \vec{g}\right) \\
& +\sum_{p=1}^{n} K_{p q}\left(\vec{v}_{p}-\vec{v}_{q}\right)+\dot{m}_{p q} \vec{v}_{p q}-\dot{m}_{q p} \vec{v}_{q p} \\
& +\vec{F}_{q}+\vec{F}_{l i f t, q}+\vec{F}_{w l, q}+\vec{F}_{v m, q}+\vec{F}_{t d, q}+\vec{F}_{D, q}
\end{aligned}
$$

where ${ }^{\tau_{q}}$ is the $q^{\text {th }}$ phase stress-strain tensor:

$$
\overline{\bar{\tau}}_{q}=\alpha_{q} \mu_{q}\left(\nabla \vec{v}_{q}+\nabla \vec{v}_{q}{ }^{T}\right)+\alpha_{q}\left(\lambda_{q}-\frac{2}{3} \mu_{q}\right) \nabla \cdot \vec{v}_{q}
$$

where $\mu_{q}$ and $\lambda_{q}$ are the shear and bulk viscosity of phase $q, \vec{g}$ is the acceleration of gravity; $\vec{F}_{q}$ is the $q^{\text {th }}$ phase external body force; $\vec{F}_{l i f t, q}$ is the $q^{\text {th }}$ phase lift force $; \vec{F}_{w l, q}$ is the $q^{\text {th }}$ phase wall lubrication force; $\vec{F}_{v m, q}$ is the $q^{\text {th }}$ phase virtual mass force; $\vec{F}_{t d, q}$ is the $q^{\text {th }}$ phase turbulent dispersion force; $\vec{F}_{D, q}$ is the $q^{\text {th }}$ phase drag force; $\vec{v}_{p q}$ is the interphase velocity; and $K_{p q}$ is the momentum exchange coefficient between each phase as defined in (Kolev 2007, 2011).

In the two-phase flow, the secondary phase (in this case the vapor phase) is assumed to form droplets or bubbles. For the current study, the secondary phase bubbles are described as deformable in the ellipsoidal and spherical cap regimes 
(Zeitoun and Shoukri 1996). Drew (Drew 1983) pointed out that the central aspect of two-phase flows that makes them fundamentally different from a single-phase flow is the interracial interaction terms; in the absence of an interfacial mass transfer, the difference is contained in the interfacial forces. There are some applicable models in existence which have removed any possible inconsistencies or untoward predictions in their values. Based on the stated assumption, the interphase momentum exchange variables are modeled in the next sections.

\subsubsection{Interphase drag force $(\quad)$}

As a result of the reciprocating motion, there will be a constant deformation of the hydraulic boundary layer, thermal layer, and the bubble shapes. The bubbles can have a range of shapes such as spherical, elliptical, or cap. As a result, the steady-state

condition of in the two-phase flow is modeled in terms of the drag coefficient $C_{D}$ and relative phase velocity $v_{r}$ using The Grace model(Clift, Grace, and Weber 2005):

$$
\vec{F}_{D, q}=\frac{C_{D}}{24} \frac{d_{p} \rho_{q}\left|v_{r}\right|}{\mu_{q}}
$$

where $C_{D}, v_{r}$ vary with flow regime as prescribed by (Ishii and Zuber 1979) and (Clift, Grace, and Weber 2005).

\subsubsection{Interphase lift force ( )}

The secondary phase will experience lift forces mainly due to velocity gradients in the primary-phase (liquid phase) flow field. In most cases, the lift force is 
insignificant compared to the drag force. In this study where the phases separate quickly, the lift force is significant. $\vec{F}_{\text {lift }}$ is suitably modeled according to the work by Frank et al. (Frank, Shi, and Burns 2004), which is a modification of the Tomiyama lift model (Tomiyama 1998). The main feature of this model is the prediction of the crossover point in bubble size at which particle distortion causes a reversal in the sign of $\vec{F}_{l i f t}$. The model is expressed as follows:

$$
\vec{F}_{\text {lift }}=C_{l} \alpha_{p} \rho_{q} v_{r} \times\left(\nabla \times v_{l}\right)
$$

where $C_{l}$ is the lift force coefficient (Frank, Shi, and Burns 2004; Tomiyama 1998).

\subsubsection{Turbulent dispersion force ( )}

For boiling flows, the vapor (stream of bubbles) formed at the wall, moves through the turbulent primary fluid and spreads out. This spreading, or dispersion, is a collective result of the random motions of the primary fluid and dispersed secondary phase. $\vec{F}_{t d}$ is essential for transporting the stream of bubbles from walls to the core fluid flow regions and results in the diffusion of the vapor phase caused by liquid phase turbulence (Drew 1983). The classic model of $\vec{F}_{t d}$ requires an approximation of the drift velocity. The reciprocating motion of the fluid in this study makes this task more complicated. As a result, this study adopted the model of Lopez de Bertodano (Lopez de Bertodano 1992), which models $\vec{F}_{t d}$ in terms of $\rho_{q}$, the turbulent kinetic energy in 
the continuous phase ${ }^{k_{q}}$, and the gradient of dispersed phase volume fraction $\nabla \alpha_{q}$, with a coefficient $C_{T D}$ :

$$
\vec{F}_{t d}=-\vec{F}_{t d}=C_{T D} \rho_{q} k_{q} \nabla \alpha_{q}
$$

\subsubsection{Wall lubrication force ( )}

Wall lubrication force on the secondary phase acts in the radial direction by pushing the bubbles away from the wall and prevents their accumulation near the wall. This force results in the vapor phase concentrating in a region near, but not immediately adjacent to, the wall. has the general form:

$$
\vec{F}_{w l}=C_{w l} \rho_{q} \alpha_{P}\left|v_{r \|}\right|^{2} \vec{n}_{w}
$$

where $v_{r \|}$ is the phase relative velocity component tangential to the wall surface, ${ }^{w}$ the normal unit vector pointing away from the wall, and $C_{w l}$ is the wall lubrication coefficient that is a modification of the wall lubrication force formulation of Antal et al. (Antal, Lahey, and Flaherty 1991; Kolev 2011).

\subsubsection{Virtual mass force ( )}

The virtual mass force is experienced by the body as if it had an additional mass during its translation relative to the continuum (Kolev 2007). In this case, the virtual mass effect is significant because the secondary phase density is smaller than the primary phase density. As a result, the inertia of the liquid-phase mass encountered by 
the accelerating particles (or droplets or bubbles) of the vapor phase exerts a "virtual mass force" on the particles (Drew 1983). The general form of the virtual mass force is given as (Kolev 2011):

$$
\vec{F}_{v m}=C^{v m} \alpha_{p} \rho_{p}\left[\left(\frac{\partial \vec{u}_{v}}{\partial t}+\vec{u}_{v} \cdot \nabla \vec{u}_{v}\right)-\left(\frac{\partial \vec{u}_{l}}{\partial t}+\vec{u}_{l} \cdot \nabla \vec{u}_{l}\right)\right]
$$

where the virtual mass coefficient $C^{v m}$ is set to 0.5 as recommended in (Kolev 2011)

\subsubsection{Transport effects at the interface}

A surface of discontinuity, as an interface, is associated with the formation of bubbles due to heat transfer from the solid wall to the coolant and disintegration of the bubbles due to heat transfer from the bubbles as they disperse from the wall to the subcooled region in the primary phase. When considering the appropriate interface jump, conditions relating the values of the fundamental quantities on both sides of the interface should be considered (Faghri and Zhang 2006). For this study, the energy and mass relations at the non-flat liquid-vapor interface are approximated as presented in the following sections.

\subsubsection{Interface to Liquid Heat Transfer ( , )}

Based on the classic Ranz-Marshall correlation (Mashayek 2001), the heat transfer $\left(q_{l t}\right)$ from the bubble interface to the liquid is defined as: 


$$
\begin{aligned}
& q_{l t}=h_{s l} A_{i}\left(T_{s a t}-T_{l}\right) \\
& h_{s l}=\frac{k_{l}}{D_{b}}\left(2+0.6 \operatorname{Re}^{0.5}+\operatorname{Pr}^{0.33}\right)
\end{aligned}
$$

\subsubsection{Interface to Vapor Heat Transfer ( )}

The interface to vapor heat transfer is calculated using the constant time scale return to saturation method (Tentner, Merzari, and Vegendla 2014; H. Li et al. 2011). The formulation is as follows:

$$
q_{v t}=\frac{\alpha_{v} \rho_{v} C_{p_{v}}}{.05}\left(T_{s a t}-T_{v}\right)
$$

\subsubsection{Interfacial Mass Transfer}

Assuming that all the heat transferred to the interface is associated with phase change, the formulation for the interfacial mass transfer rate is as follows:

$$
\dot{m}_{E}=\frac{q_{l t}+q_{v t}}{h_{f v}}
$$

\subsubsection{Other closure parameters}

The following parameters, interphase contact area, area of influence ${ }_{b}$, the frequency of bubble departure $f$, nucleate site density ${ }^{N}$, and bubble departure diameter $D_{W}$, provide closure for the preceding equations. Values for these parameters were obtained based on (Aminfar, Mohammadporfard, and Maroofiazar 2013).The 
mean bubble diameter $d_{b}$ that is estimated from the mean Sauter bubble diameter of Zeitoun and Shoukri (Zeitoun and Shoukri 1996) is employed in this study.

\subsection{Turbulence modeling}

The turbulence parameters are obtained from the following transport equations (David C. Wilcox 2006; Kim, Choudhury, and Patel 1999; Yakhot and Smith 1992; Shih et al. 1995; F R Menter 1994; Walters and Cokljat 2008; R. B. Langtry et al. 2006):

\subsubsection{Standard k- $\varepsilon$ Model}

The turbulence kinetic energy $(k)$ and turbulence rate of dissipation $(\varepsilon)$ are given as follows:

$$
\begin{aligned}
& \frac{\partial}{\partial t}(\rho k)+\frac{\partial}{\partial x_{i}}\left(\rho k u_{i}\right)=\frac{\partial}{\partial x_{j}}\left[\left(\mu+\frac{\mu_{t}}{\sigma_{k} t}\right) \frac{\partial k}{\partial x_{j}}\right]+G_{k}+G_{b}-\rho \varepsilon-Y_{M}+S_{k} \\
& \frac{\partial}{\partial t}(\rho \varepsilon)+\frac{\partial}{\partial x_{i}}\left(\rho \varepsilon u_{i}\right)=\frac{\partial}{\partial x_{j}}\left[\left(\mu+\frac{\mu_{t}}{\sigma_{\varepsilon} t}\right) \frac{\partial \varepsilon}{\partial x_{j}}\right]+C_{1 \varepsilon} \frac{\varepsilon}{k}\left(G_{k}+C_{3 \varepsilon} G_{b}\right)-C_{2 \varepsilon} \rho \frac{\varepsilon^{2}}{k}+S_{\varepsilon}
\end{aligned}
$$

In these equations, ${ }^{G_{k}}$ represents the generation of turbulence kinetic energy due to the mean velocity gradients; $G_{b}$ is the generation of turbulence kinetic energy due to buoyancy, ${ }^{G_{k}}$ represents the contribution of the fluctuating dilatation incompressible turbulence to the overall dissipation rate, and ${ }^{C_{1 \varepsilon}}, C_{2 \varepsilon}$, and ${ }^{C_{3 \varepsilon}}$ are the related empirical constants (Henkes, Van Der Vlugt, and Hoogendoorn 1991). The value of $C_{1 \varepsilon}$ and $C_{2 \varepsilon}$ 
are set respectively at 1.44 and 1.92. ${ }^{\sigma_{k}}$ and ${ }^{\sigma_{\varepsilon}}$ are the turbulent Prandtl numbers for $k$ and $\varepsilon$ respectively with values 1.0 and 1.3 respectively, $S_{k}$ and $S_{\varepsilon}$ are source terms which in this study are set to be zero. Additional information on value selection and modeling equations of $G_{k} G_{b} G_{k}$ and $C_{3 \varepsilon}$ are presented in Launder and Spalding 1974. The turbulent viscosity $\mu_{t}$ is modeled as follows,

$$
\mu_{t}=\rho C_{\mu} \frac{k^{2}}{\varepsilon}
$$

and $C_{\mu}$ is set at 0.09 (Henkes, Van Der Vlugt, and Hoogendoorn 1991; Launder and Spalding 1974)

\subsubsection{RNG $k$ - $\varepsilon$ Model}

The RNG k- $\varepsilon$ Model is a modification of the standard k- $\varepsilon$ Model. It includes the additional term in its $\varepsilon$ equation, incorporating the effect of swirl on turbulence, an analytical formula for turbulent Prandtl numbers and an analytically derived differential formula for effective viscosity. In addition to the documented swirl modification, additional modifications are shown in the following formulations (Yakhot and Smith 1992):

(i) $\left(\mu+\frac{\mu_{t}}{\sigma_{k} t}\right)$ in Eq. 18 is substituted with $\alpha_{k} \cdot \mu_{\text {eff }}$
(ii) $\alpha_{\varepsilon} \cdot \mu_{\text {eff }}$ in Eq. 19 is substituted with $\left(\mu+\frac{\mu_{t}}{\sigma_{\varepsilon} t}\right)$ 
(iii)Additional $-R_{\varepsilon}$ in Eq. 18

(iv) $C_{\mu}, C_{1 \varepsilon}$ and $C_{2 \varepsilon}$ are set at $0.0845,1.42$ and 1.68 , respectively.

$\alpha_{k}$ and ${ }^{\alpha_{\varepsilon}}$ are the inverse effective Prandtl numbers for ${ }^{k}$ and $\varepsilon$ respectively, and ${ }^{\mu_{e f f}}$ is the effective viscosity (Yakhot and Smith 1992).

\subsubsection{Realizable $k-\varepsilon$ Model}

The Realizable $k$ - $\varepsilon$ Model is also a modification of the standard $k$ - $\varepsilon$ Model. It uses the same formulation for the $k$ but has an alternative formulation for $\varepsilon$ (Shih et al. 1995):

$$
\frac{\partial}{\partial t}(\rho \varepsilon)+\frac{\partial}{\partial x_{i}}\left(\rho \varepsilon u_{i}\right)=\frac{\partial}{\partial x_{j}}\left[\left(\mu+\frac{\mu_{t}}{\sigma_{\varepsilon} t}\right) \frac{\partial \varepsilon}{\partial x_{j}}\right]+\rho C_{1} S \varepsilon+C_{1 \varepsilon} \frac{\varepsilon}{k_{k}} \cdot C_{3 \varepsilon} G_{b}-\rho C_{2} \frac{\varepsilon^{2}}{k+\sqrt{v \varepsilon}}+S_{\varepsilon}
$$

where $C_{1}$ and $C_{2}$ are empirical constants (Shih et al. 1995).

\subsubsection{Standard $k$ - $\omega$}

The transport equations for $k$ and the specific dissipation rate $(\omega)$ are as follows (Florian R. Menter 2011):

$$
\begin{aligned}
& \frac{\partial}{\partial t}(\rho k)+\frac{\partial}{\partial x_{i}}\left(\rho k u_{i}\right)=\frac{\partial}{\partial x_{j}}\left[\Gamma_{k} \frac{\partial k}{\partial x_{j}}\right]+G_{k}-Y_{k}+S_{k} \\
& \frac{\partial}{\partial t}(\rho \omega)+\frac{\partial}{\partial x_{i}}\left(\rho \omega u_{i}\right)=\frac{\partial}{\partial x_{j}}\left[\Gamma_{\omega} \frac{\partial \omega}{\partial x_{j}}\right]+G_{\omega}+Y_{\omega}+S_{\omega}
\end{aligned}
$$

In these equations, ${ }^{G_{k}}$ represents the generation of turbulence kinetic energy due to the mean velocity gradients, ${ }^{\omega}$ represents the generation of $\omega, \Gamma_{k}$ and $\Gamma_{\omega}$ represent the 
effective diffusivity of $k$ and $\omega$ respectively, $Y_{k}$ and $Y_{\omega}$ represent the dissipation of $k$ and $\omega$ due to turbulence. All of the preceding terms are calculated and specified in (Henkes, Van Der Vlugt, and Hoogendoorn 1991; Florian R. Menter 2011).

\subsubsection{Shear-Stress Transport (SST) $k$ - $\omega$ Model.}

The SST $k$ - $\omega$ model is a modification of the standard $k$ - $\omega$ model. The modifications are as follows (F R Menter 1994):

(i) $G_{k}$ in Eq. 22 is substituted with $\tilde{G}_{k}$

(ii) Additional $D_{\omega}$ is added to Eq. 23

${ }^{G_{k}}$ represents the generation of turbulence kinetic energy due to mean velocity gradients, calculated from ${ }_{k}, D_{\omega}$ represents the cross-diffusion term (Henkes, Van Der Vlugt, and Hoogendoorn 1991; F R Menter 1994), and $S_{k}$ and $S_{\omega}$ are source terms which in this study are set to be zero.

\subsubsection{Transition $k-k_{L}-\omega$}

For an incompressible single-phase flow with no body forces, governed by the steady Reynolds-averaged continuity and momentum equations as well as a linear eddy-viscosity model for the Reynolds stresses, the $k-\quad-\omega$ governing equations for turbulent kinetic energy $k$, the laminar kinetic energy , and the scale-determining variable $\omega$ are as follows(Walters and Cokljat 2008):

$$
\frac{D k}{D t}=P_{k}+R_{B P}+R_{N A T}-\omega k-D_{T}+\frac{\partial}{\partial x_{j}}\left[\left(v+\frac{\alpha_{T}}{\alpha_{k}}\right) \frac{\partial k}{\partial x_{j}}\right]
$$




$$
\begin{gathered}
\frac{D k_{L}}{D t}=P_{k L}+R_{B P}+R_{N A T}-\omega k-D_{L}+\frac{\partial}{\partial x_{j}}\left[v \frac{\partial k_{L}}{\partial x_{j}}\right] \\
\frac{D \omega}{D t}=C_{\omega l} \frac{\omega}{k} P_{k}+\left(\frac{C_{\omega R}}{f_{W}}-1\right) \frac{\omega}{k}\left(R_{B P}+R_{N A T}\right)-C_{\omega 2} \omega^{2}+C_{\omega 3} f_{\omega} \alpha_{T} f_{W}^{2} \frac{\sqrt{k}}{d^{3}}+\left[\left(v+\frac{\alpha_{T}}{\sigma_{\omega}}\right) \frac{\partial \omega}{\partial x_{j}}\right]
\end{gathered}
$$

The various terms in the model equations represent production, destruction, and transport mechanisms (Walters and Cokljat 2008; S.-E. Kim, Choudhury, and Patel 1999).

\subsubsection{Transition SST Model;}

The transition SST model is based on the coupling of the SST $k$ - $\omega$ model with two other transport equations, one for the intermittency and one for the transition onset criteria, in terms of momentum-thickness Reynolds number. The transport equations for the intermittency $\gamma$ and the various terms in the model equations representing production, destruction, and transport mechanisms are provided in (R. B. Langtry et al. 2006; F. R. Menter et al. 2006; R. Langtry and Menter 2005).

\subsection{Boundary Conditions:}

The general external boundary conditions for the numerical modeling have been presented in Fig. 4.4, and additional specific boundary conditions are given as follows: Inlet:

$$
V_{\text {out }}=V_{\text {in }}, \quad T_{\text {in }}=\left(T_{\text {out }}\right)_{\text {ave }}
$$

Outlet: 


$$
P=P_{\text {out }}, \quad \frac{\partial T}{\partial n}=0
$$

Fluid-solid interface:

$$
\vec{V}=0, \quad T=T_{s}, \quad-k_{s} \frac{\partial T_{s}}{\partial n}=-k \frac{\partial T}{\partial n}
$$

Top wall:

$$
q_{w}=-k_{s} \frac{\partial T_{s}}{\partial n}
$$

In Eqs. (36-38), $V_{\text {in }}$ and $T_{\text {in }}$ are the fluid inlet velocity and temperature, respectively,

$p_{\text {out }}$ is the pressure at the outlet, $n$ is the direction normal to the wall or the outlet plane, and $q_{w}$ is the heat flux applied to the wall of the heat source or sink.

\subsection{CAD Meshing, Discretization and Simplification}

Using Solidworks, the geometry for various configurations of the RMDHL was developed. Fig 4.7 to Fig 4.9 shows the CADs developed for all three generations of the RMDHL. The CAD developed in Solidworks is exported into ANSYS for computational analyses. In ANSYS the solids that are not required for the analysis are suppressed as shown in Fig 4.10. Meshing for the geometrical model in Fig. 4.10 is generated for the solid and fluid domains as illustrated in Fig 4.11. 


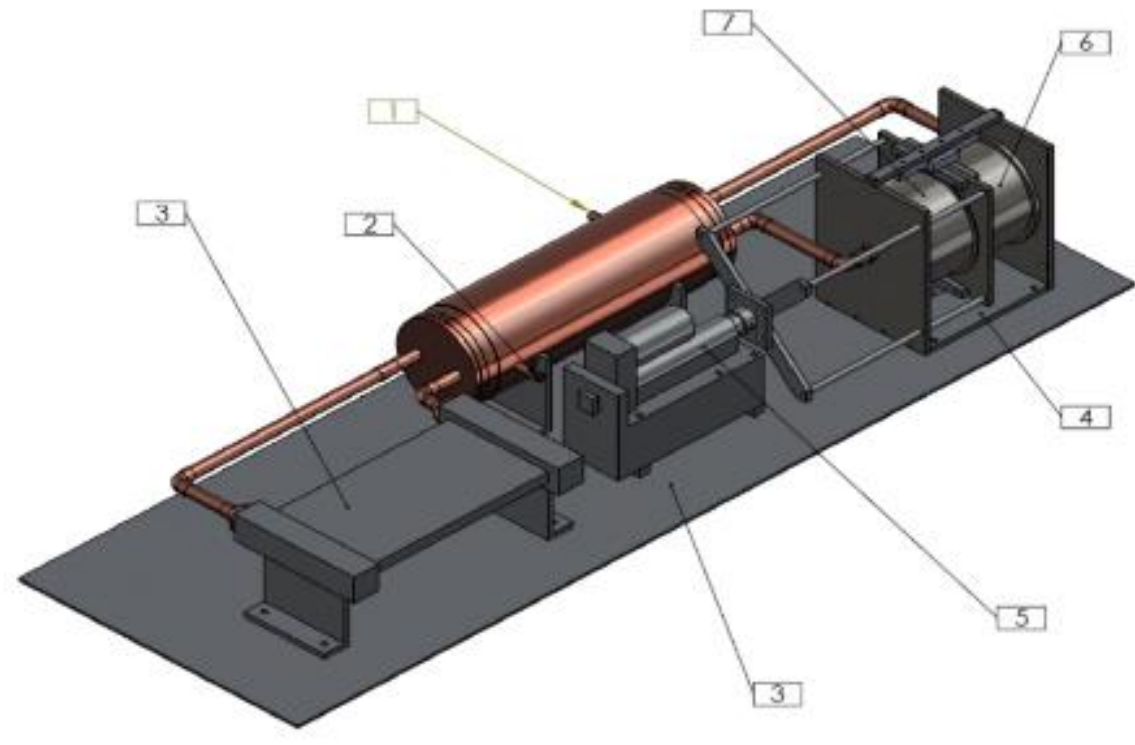

Figure 4. 7: CAD developed for first generation bellows type RMDHL

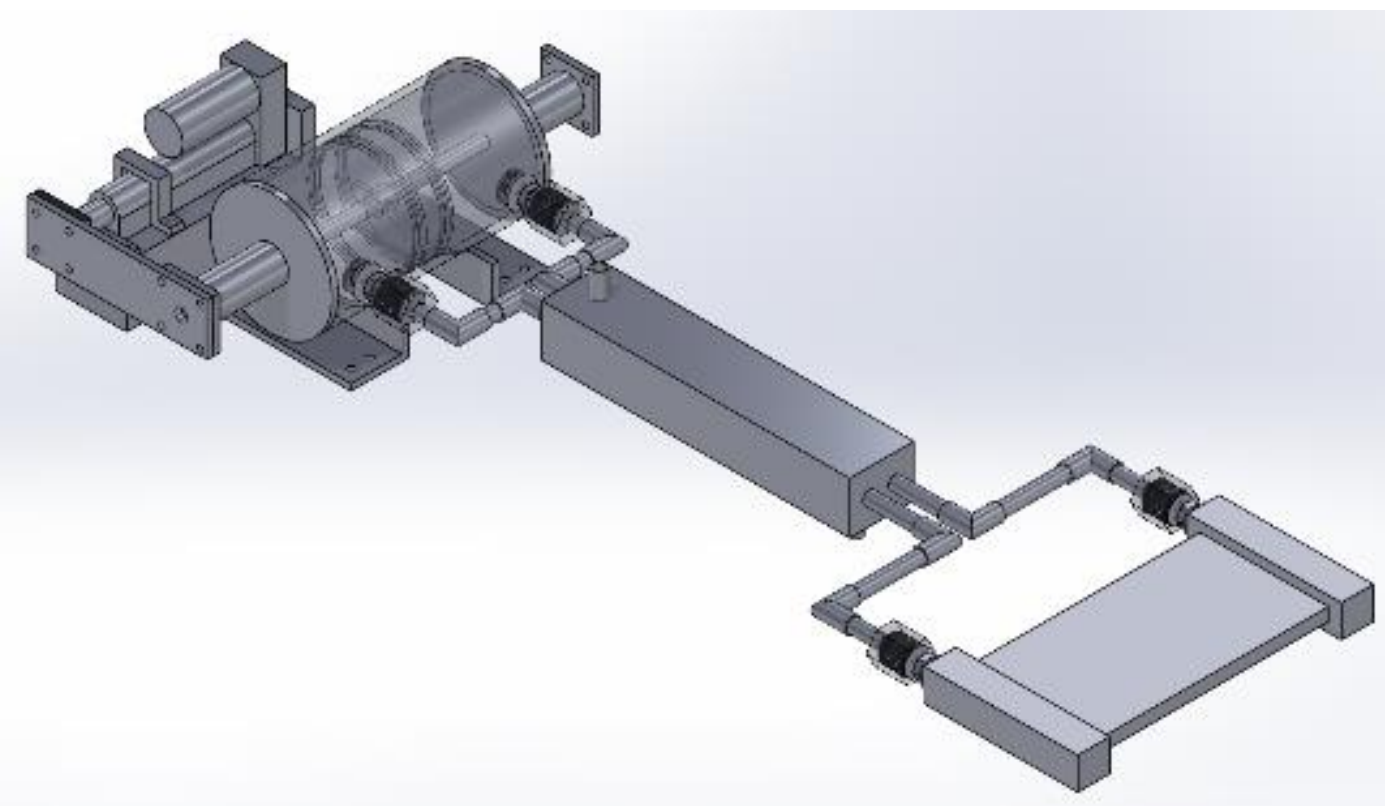

Figure 4. 8: CAD developed for second generation Bellows Type RMDHL 


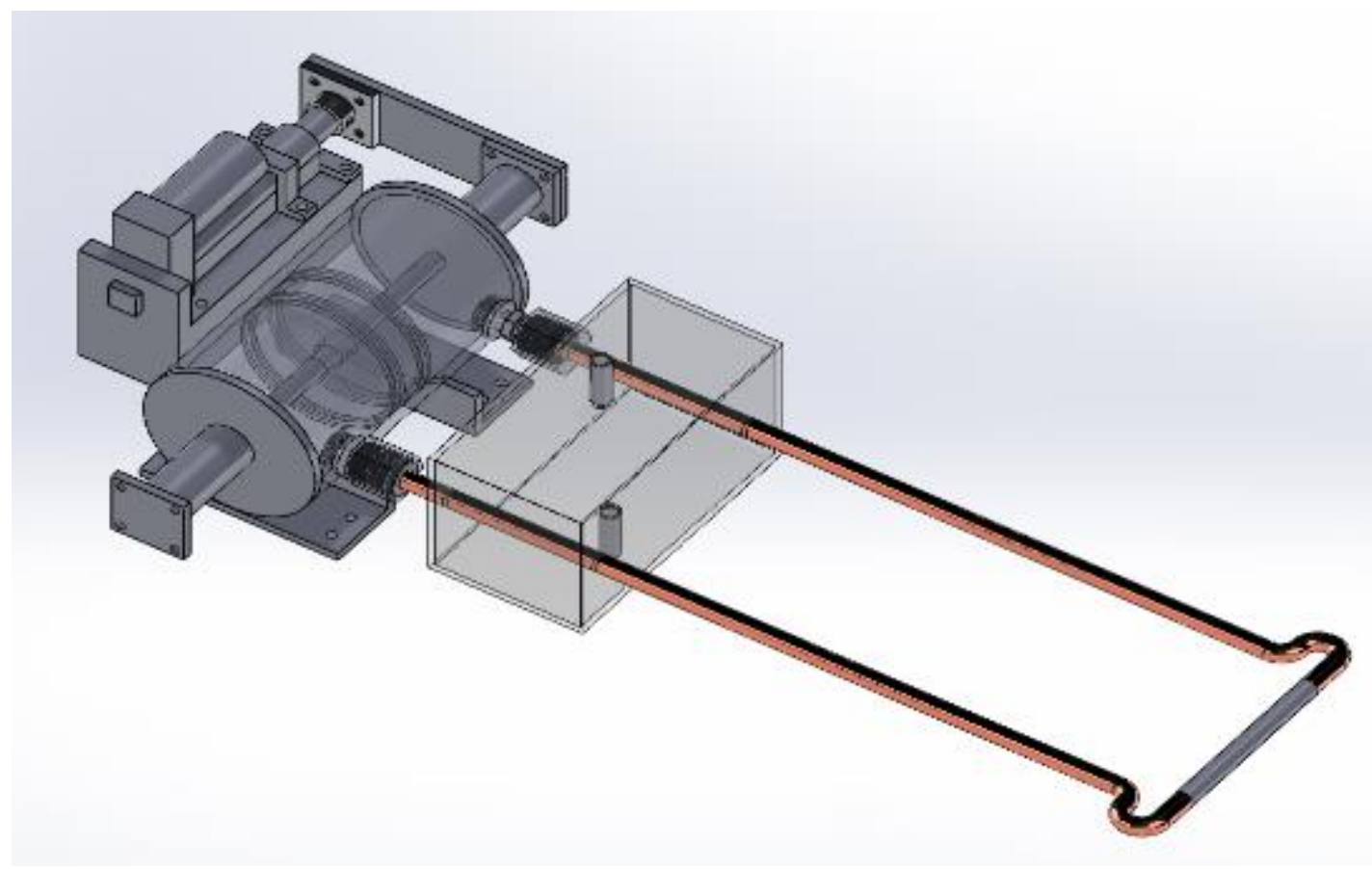

Figure 4. 9: CAD developed for third generation bellows type RMDHL 


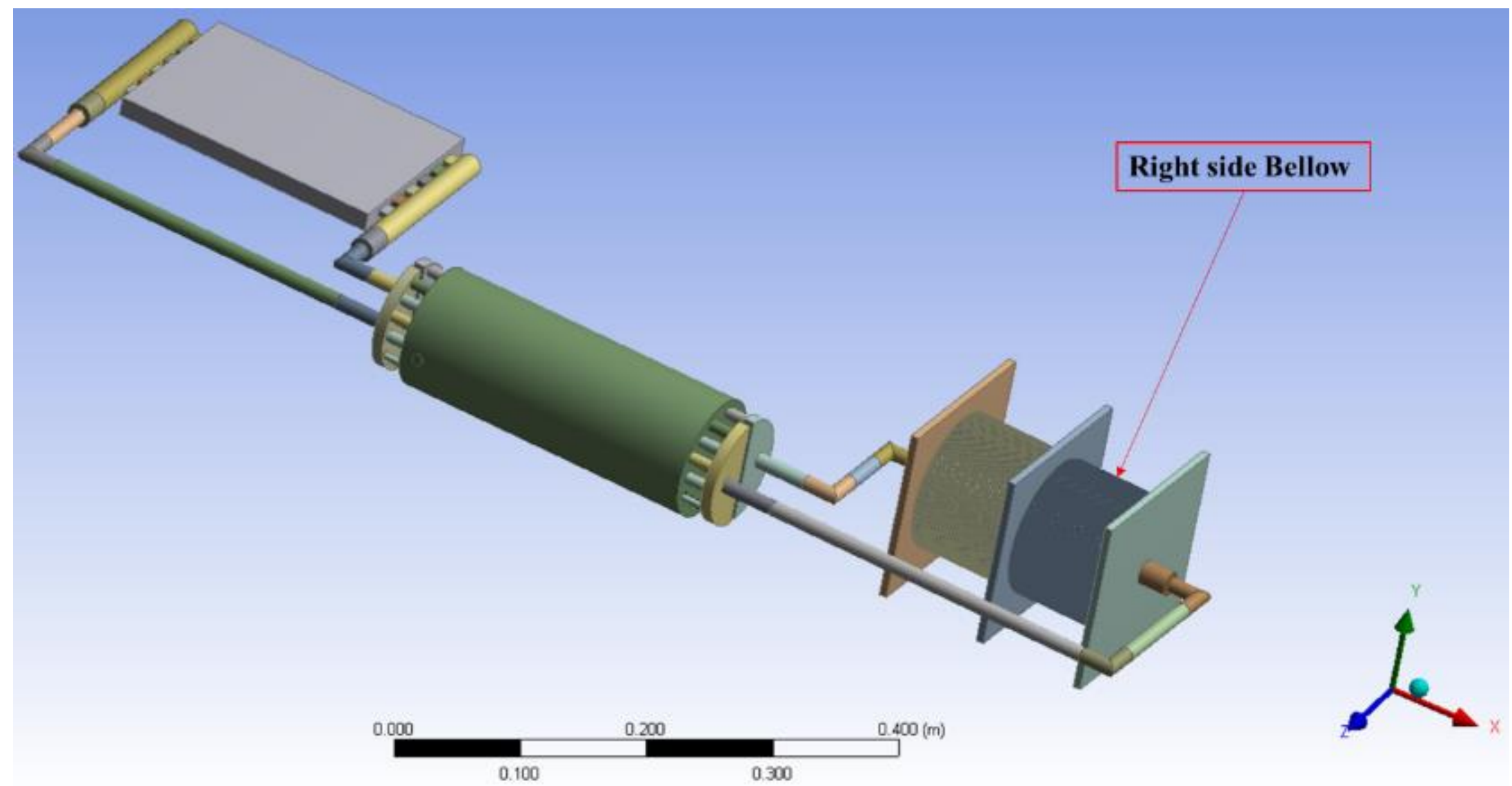

Figure 4. 10: Geometrical Model of Bellows - type RMDHL indicating one of the bellows and suppressed solid sections 


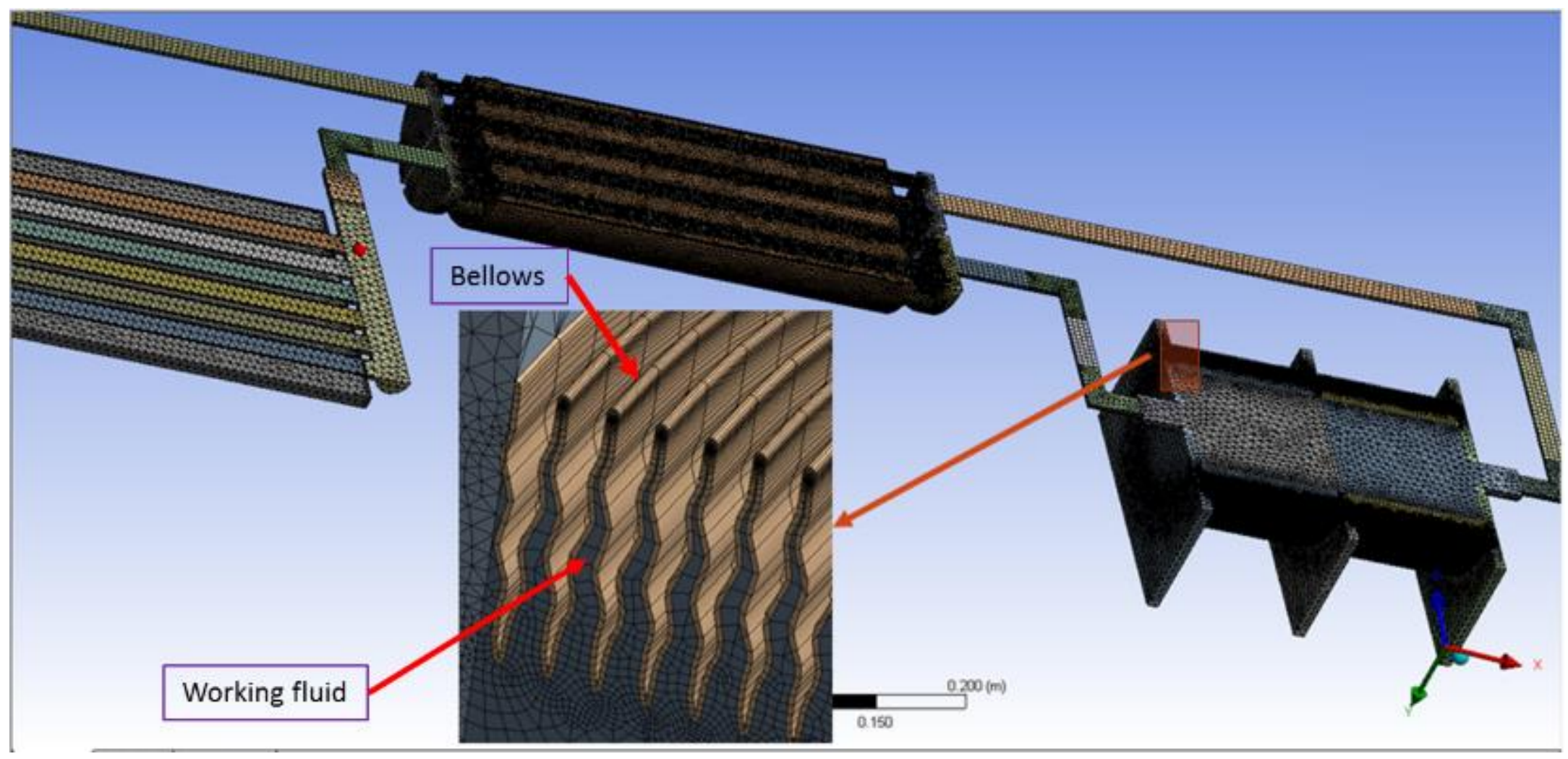

Figure 4. 11: Meshes of the geometrical model of the right bellows of the RMDHL 


\subsection{Virtual Loop}

The focus of the computational fluid dynamics modeling is the fluid behavior and heat transfer characteristics of the system of interest. The physical geometry of the loop plays a critical role in defining the boundary conditions. In this study, the control volume is defined by the interior of the solids and control surfaces are specified as inlets and outlets. Irrespective of the problem, the boundary conditions need to be defined while the solids and fluids are treated with meshes points. Each of these mesh points requires computational resources to resolve. For instance, the mesh of only the right bellow has 1,132,096 nodes and 1,064,379 elements whereas the combined node and elements for the rest of the loop (excluding both bellows) are less than 1,000,000. Two-thirds of the computational cost will be associated with resolving the bellows while only one third will be dedicated to the region of interest.

To optimized computational resources, the usual practice in general CFD modeling is to treat solids as walls and focus on the fluid in the region of interest. For the RMDHL, this becomes a challenge for two reasons:

1. In specifying inlet and outlet boundaries: Unlike unidirectional flow, the inlet and outlet interchange with the direction of the flow, and the outlet and the inlet in the forward direction become the inlet and outlet in the reverse direction.

2. the fluid conditions at the outlet and inlet of the model are interdependent for both the forward and reverse flow: the outlet flow conditions determine the value of the inlet flow conditions.

3. Complexities associated with geometry creates by the bellows. 
A literature review of earlier numerical studies did not provide details on how to resolve the inlet and outlet boundary condition of the oscillating flow. A few studies directly modeled the loop driver using a fluid-structure interaction (FSI) model. In FSI the solid motion is modeled, and fluid motion is modeled as resultants from the solid motion. As a result of the complexity of the bellows geometry, FSI for the RMDHL requires costly computational resources.

In order to avoid devoting scarce computational resources to this complexity, this study developed a virtual loop for both single phase and two-phase RMDHL loops (appendix A and Appendix B). A virtual loop is schematically shown in Fig. 4. 12 and was developed based on the following assumptions:

1. The function of the motor-driven mechanical driver of the RMDHL is to keep the working fluid circulating through the loop.

2. The driver provides the oscillating pressure differential within the loop which moves the cooling fluid back and forth within the loop.

3. To prevent a possibility of the occurrence of cavitation, it is essential that the portion of the working fluid that is vaporized at the cold plate be wholly condensed into the liquid before the fluid is recirculated in the condenser.

4. The working fluid enters the driver as liquid and exits as a liquid.

5. The pressure increase by the driver does not make any significant contribution to phase change.

6. Working fluid is incompressible within the driver.

7. Conditions within the driver are isobaric, adiabatic, isochoric and isentropic. 


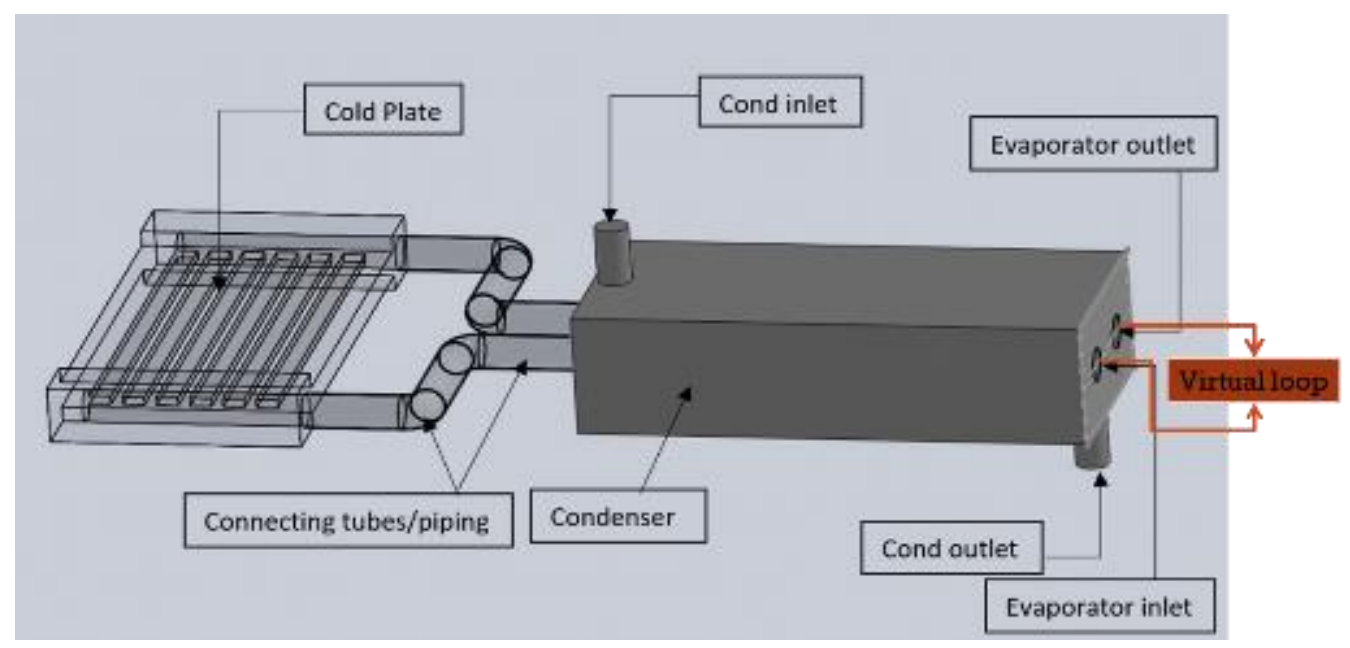

(a)

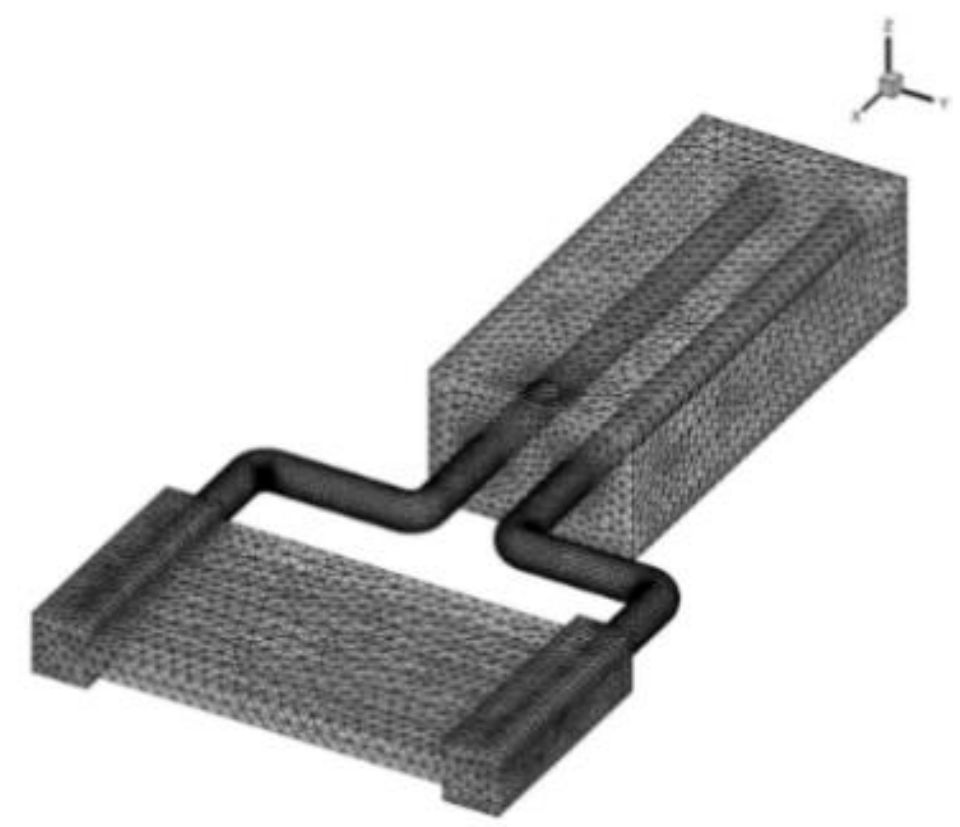

(b)

Figure 4. 12: (a) Geometry of the RMDH loop used for numerical model (b) Grid distribution for the RMDH loop 
The UDF.h header in Appendix 3 contains definitions for macros as well as compiler directives for $\mathrm{C}$ library function header files used in the Virtual loop. Appendix 3 also includes header files for other Ansys Fluent-supplied macros and functions. The virtual loop is a routine written in $\mathrm{C}$ which is dynamically linked with the solver. It is used to determine the interfacial conditions between the end of the condenser and the pump. In this case, only a constant average temperature is initiated for the computation process, and the final working fluid temperature at the interface had been determined through the iteration of the computational procedure. Even if physically the loop is open as depicted in Fig 4.12, the user-defined function has closed the loop from the computational point of view. The virtual loop is used to reduce the computational cost of solving the whole domain and also to avoid the simulation of the solenoid part which requires an FSI modeling. The constant heat flux condition is applied to the outer surface of the cold plate while the rest of the exterior surface of the loop was insulated. A constant temperature is applied at the inlet of the condenser cooling water

\subsection{Empirical method for comparing experimental data and Numerical}

\section{Results}

A numerical model calibration template (Moriasi et al. 2007) is adapted for comparing and ranking numerical model predictions for the cold plate temperature with measured data. The template investigates the accuracy of each numerical prediction and then benchmarks each numerical result against data. The template adopts $y$ - 
intercept of the best-fit regression (Pearson's correlation coefficient ( $\mathrm{r}$ ) and coefficient of determination $\left(\mathrm{R}^{2}\right)$ ), the Nash-Sutcliffe efficiency (NSE) presented in Eq. (39), the percentage bias (PBIAS) presented in Eq. Error! Reference source not found. and RMSE-observations standard deviation ratio (RSR) presented in Eq. (41).

NSE determines the relative magnitude of the residual variance ("noise") compared to the measured data variance ("information"). NSE ranges between $-\infty$ and 1.0, the closer NSE is to 1 , the more accurate the prediction. PBIAS measures the tendency of the simulated data to be larger or smaller than the experimental data, the lower the magnitude, the more accurate the model simulation, and the optimal value would, therefore, be zero. RSR measures the error index, and like the PBIAS, its optimal value is zero.

$$
\begin{gathered}
N S E=1-\frac{\sum_{i=1}^{n}\left(Y_{i}^{\text {exp }}-Y_{i}^{\text {sim }}\right)^{2}}{\sum_{i=1}^{n}\left(Y_{i}^{\text {exp }}-Y_{i}^{\text {mean }}\right)^{2}} \\
\text { PBIAS }=\frac{\sum_{i=1}^{n}\left(Y_{i}^{\text {exp }}-Y_{i}^{\text {sim }}\right) \times(100)}{\sum_{i=1}^{n}\left(Y_{i}^{\text {exp }}\right)} \\
R S R=\frac{\sqrt{\sqrt{\sum_{i=1}^{n}\left(Y_{i}^{\text {exp }}-Y_{i}^{\text {sim }}\right)^{2}}}}{\sqrt{\sum_{i=1}^{n}\left(Y_{i}^{\text {exp }}-Y_{i}^{\text {mean }}\right)^{2}}}
\end{gathered}
$$




\subsection{Velocity profile}

\subsubsection{Inlet/outlet conditions specifications}

The displacement of the piston $x_{p}(t)$ with time is (De-Jongh and Rijs 2004):

$$
x_{p}(t)=-1 / 2 S-1 / 2 " S \cos (\omega t)
$$

where $\omega$ is the pump frequency in radians per second. Differentiating Eq. 1 with respect to time we have the mean velocity for the oscillating flow

$$
u=u_{\max } \sin (\omega t)
$$

The stroke frequency $n$ (in strokes or cycles per second) then follows from:

$$
n=\omega /(2 \pi)
$$

The average volume flow rate $q_{a v}$ is equal to the product of stroke volume and pump frequency (De-Jongh and Rijs 2004):

$$
q_{a v}=\omega /(2 \pi) S A_{p}
$$

The pressure gradient takes the form:

$$
-\frac{1}{\rho} \frac{\partial p}{\partial x}=a e^{i \omega t}
$$

The definition of the critical Reynolds number for the reciprocating flow in the main pipe is given by: 


$$
\operatorname{Re}_{\omega}=\frac{\omega D^{2}}{v}
$$

The velocity profile for the oscillating motion of the fluid in a pipe which is driven by a sinusoidal pressure gradient was integrated over the cross-section of a pipe as given by Eq. (2) and simplified for the Reynolds number range covering the value obtained in Eq. (6) (T. S. Zhao and Cheng 1998b) and (Uchida 1956):

$$
\begin{aligned}
& \overline{\bar{V}}=\frac{V_{\max }}{\tau R} \int_{00}^{R \tau} \int_{0}\left(\frac{32}{r \sigma R e_{\omega}}\left[\sin t-\left(e^{-E}\right) \sin (t-E)\right]\right) \text { and } E=\left[1-\left(\frac{r}{R}\right)^{2}\right] \sqrt{\frac{R e_{\omega}}{8}} \\
& \sigma=\frac{8}{\alpha^{3}} \sqrt{\left(\alpha-2 C_{1}\right)^{2}+4 C_{2}^{2}}
\end{aligned}
$$

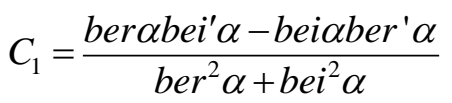

$$
\begin{aligned}
& C_{2}=\frac{\text { ber } \alpha b e i^{\prime} \alpha+b e i \alpha b e r^{\prime} \alpha}{b e r^{2} \alpha+b e i^{2} \alpha}
\end{aligned}
$$

ber( ), bei( ), $\operatorname{ber}^{\prime}\left(\right.$ ) and $\operatorname{bei}^{\prime}($ ) are kelvin functions (Abramowitz and Irene A.

Stegun 1972).

For reciprocating flow in the rectangular duct, the analytical solution for the flow between two parallel planes when there is a pressure gradient which varies harmonically with time is given by Landau and Lifshitz (Landau and Lifshitz 1987) as:

$$
\frac{\partial V}{\partial t}=a e^{i \omega t}+v \frac{\partial^{2} V}{\partial y^{2}}
$$

Subject to boundary conditions $v=0$ for $y= \pm \mathrm{h}$. If the $x z$ plane is half-way between the two planes, the solution can be rewritten in terms of the pump parameters as: 


$$
\begin{aligned}
& k=\frac{\alpha(i+1)}{H \sqrt{2}} \\
& i \omega=v k^{2} \\
& V=\frac{i a}{\omega} e^{i \omega t}\left(1-\frac{\cos k y}{\cos \frac{k H}{2}}\right) \\
& a=\pi n S=\frac{\omega S}{2}
\end{aligned}
$$

The mean value of the velocity over cross section is given as:

$$
\bar{V}=\operatorname{Real}\left\{\frac{i a}{\omega} e^{i \omega t}\left(1-\frac{2}{k H} \tan \frac{k H}{2}\right)\right\}
$$

The time and cross-channel-averaged velocity for one oscillation period is given by

$$
\bar{V}=\operatorname{Real}\left\{\frac{i a}{\omega} e^{i \omega t}\left(1-\frac{2}{k H} \tan \frac{k H}{2}\right)\right\}
$$

\subsubsection{Validation of Velocity profile}

Fig. Error! Reference source not found.13a is the velocity profile obtained for a twodimensional flow study by (Uchida 1956) and Fig Error! Reference source not found. $13 \mathrm{~b}$ is the result of the velocity profile for the $\mathrm{y}-\mathrm{z}$ plane of the 3-dimensional flow in this study, which is very similar to the results obtained by (Uchida 1956; T. S. Zhao and Cheng 1998a) In agreement with (T. S. Zhao and Cheng 1998a), the velocity profile has different shapes at accelerating half and decelerating half periods. The 
velocity is relatively steeper near the wall and flat towards the center. Fig. 4.14 also shows that the velocity profile has a parabolic definition along the channel.

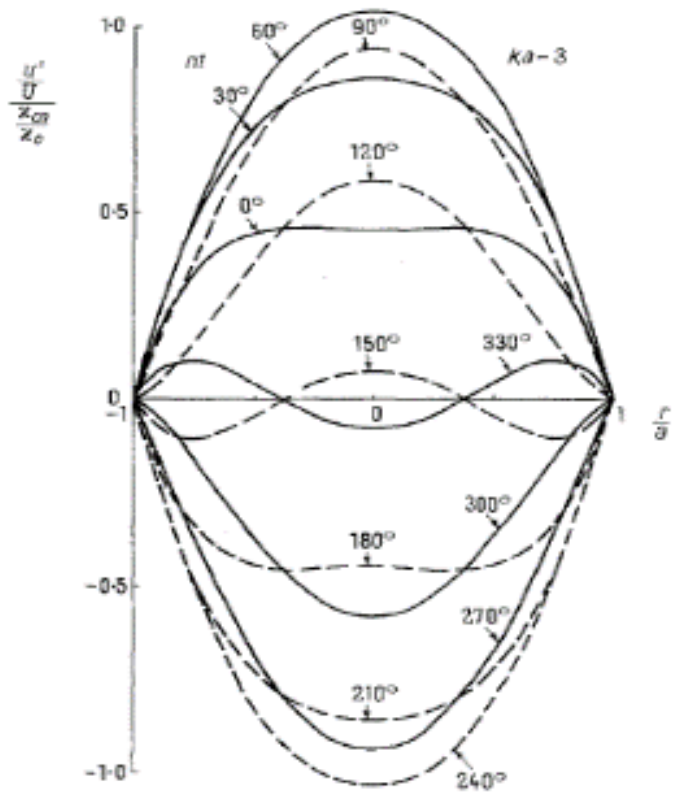

(a)

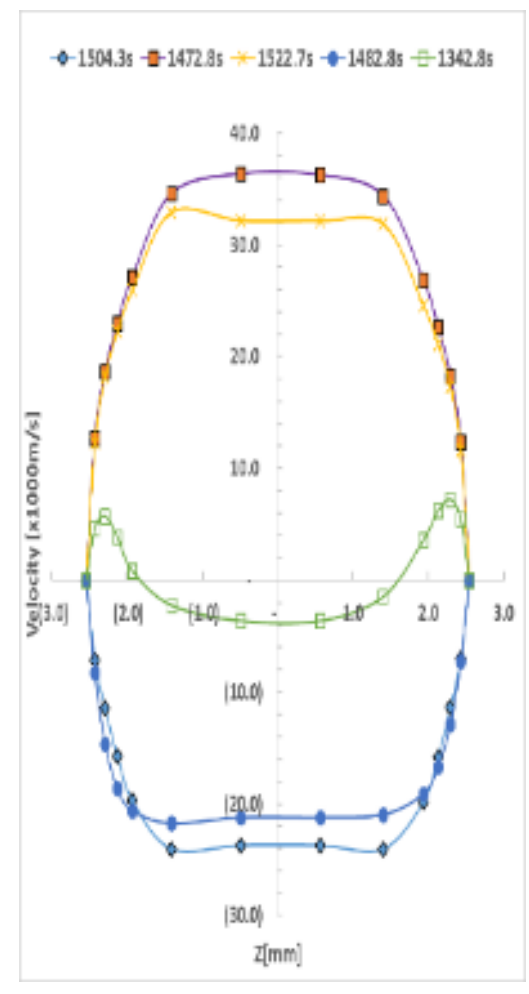

(b)

Figure 4. 13. Velocity profile for (a) pulsating viscous flow (Uchida 1956), (b) pulsating viscous flow of this study at various times 


\subsection{Comparative Studies into Temperature Uniformity between DPDHL with continuous flow Cooling and Oscillating Flow Cooling using Two-Dimensional Single-Phase Model}

The two-dimensional model of the RMDHL provides the preliminary insight into how significant difference between the temperature uniformity characteristics of the RMDHL and that of the dynamic pump driven heat loop (DPDHL) in terms of a continuous or unidirectional flow. Fig. 4:14 shows the geometry and boundary conditions of the cooling loops in the 2D analysis. The figure shows two loops, the outer loop represents the evaporator loop with the oscillating flow and the inside loop representing the condenser loop. Both loops consist of a single passage with two $90^{\circ}$ elbows. Figure 4.15 shows the grid distribution for the present simulation. The inner loop is the condenser loop, and the other loop forms the evaporator loop. The condenser and evaporator loops are enclosed with a solid wall. The two loops are separated from each other by two $0.5 \mathrm{~m}$ copper walls. The solid walls are copper, and their thermophysical properties are obtained from the fluent database. The working liquid in both loops is water. The heat fluxes are applied on both sides of the evaporator, and the boundary conditions are presented in Table 4.1.

For the present simulation about 20,000 structured elements have been used for the governing equations presented in section 4.1. For the solutions of a DPDHL, the following assumptions are also made:

(1) Both fluid flow and heat transfer are in steady-state and two dimensional.

(2) Fluid is in single phase and flow is laminar. 


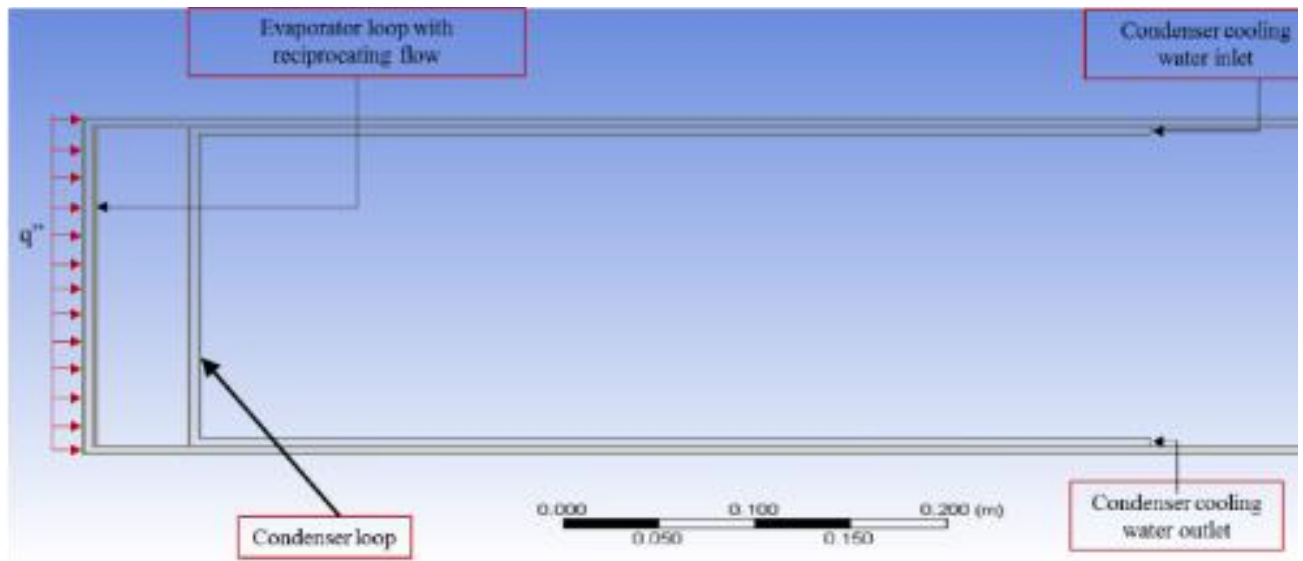

Figure 4. 14: the 2D geometry of the cooling loops.

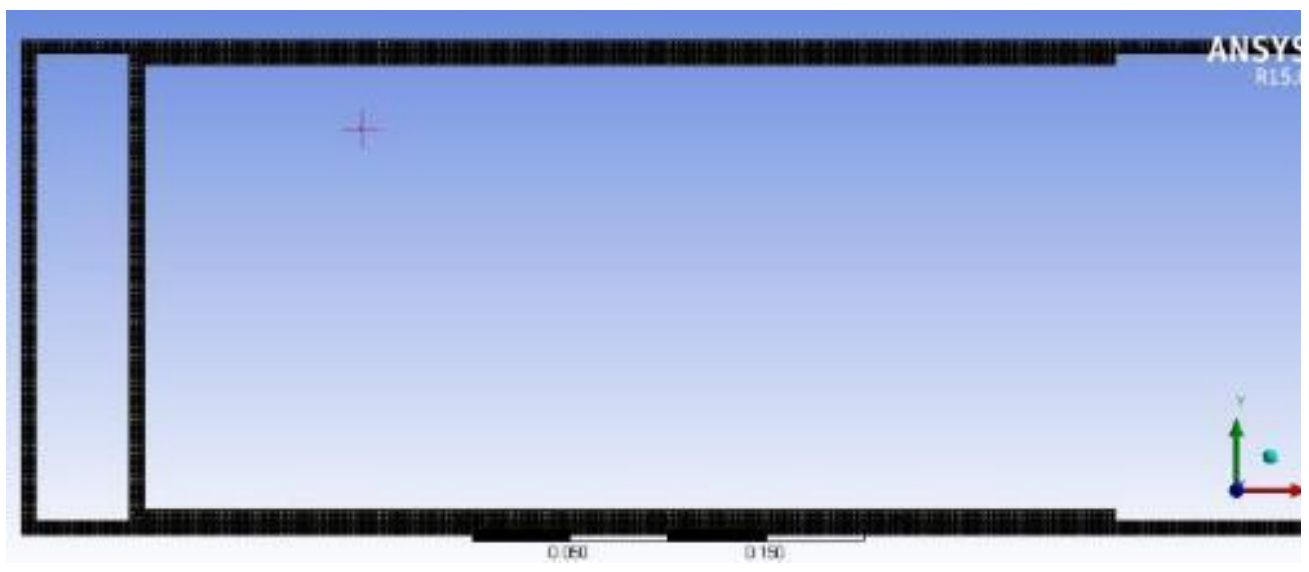

Figure 4. 15: mesh distribution for a 2D 
Table 4.1: Dimensions, geometries and boundary conditions for the present cooling loop

\begin{tabular}{|l|l|}
\hline Boundary conditions & Values and their range. \\
\hline Inlet velocity of the evaporator & $0.01,0.03 \mathrm{~m} / \mathrm{s}$ \\
\hline Inlet velocity of the condenser & $0.01,0.03 \mathrm{~m} / \mathrm{s}$ \\
\hline Heat flux on the evaporator walls & $2000,5000,10000 \mathrm{w} / \mathrm{m}^{\wedge} 2$ \\
\hline Inlet temperature of the condenser & $283 \mathrm{~K}$ \\
\hline $\begin{array}{l}\text { Channel width for both condenser and } \\
\text { evaporator loops }\end{array}$ & $0.005 \mathrm{~m}$ \\
\hline Solid wall thickness & $0.001 \mathrm{~m}$ \\
\hline
\end{tabular}


(3) Properties of both fluid and heat sink materials are temperature independent

(4) All the surfaces of heat sink exposed to the surroundings are assumed to be insulated except the walls of evaporator where constant heat flux is imposed to simulate the heat generation from the heat source.

The boundary conditions for these equations are specified in the Table 4.1. Based on the operating conditions described above, the boundary conditions for the governing equations are described by Eq (36) to Eq (38). The Geometry and grid distribution for RMDHL is the same as DPDHL. To simulate a closed-loop RMDHL, the following setup is used:

a) Instead of a closed loop, an open loop is simulated.

b) The virtual loop is used to generate the sinusoidal inlet velocity for the evaporator loop.

c) The inlet temperature of the loop is set to the average outlet temperature of the loop, and the backflow temperature of the outlet is set to the average temperature of the inlet boundary to mimic the temperature and velocity in the distribution of a closed loop.

Fig. 4.16 - Fig. 4. 21 show the temperature and pressure contours as well as velocity vectors for the DPDHL for evaporator and condenser inlet velocity of 0.01 $\mathrm{m} / \mathrm{s}$, wall flux of $2000 \mathrm{w} / \mathrm{m}^{\wedge} 2$ and inlet temperature of the condenser at $283 \mathrm{~K}$. Temperature profiles on the evaporator walls (left and right walls) are also shown in Fig 4.22- Fig 4.26. The figures clearly show the non-uniformity of the temperature 
profiles. As seen in these figures the temperature is minimum at the inlet of the evaporator and gradually increases toward the outlet of the outlet. Moreover, these figures clearly indicate that the temperature increase with the increased heat flux on the walls or decreased flow rate.

The results of RMDHL loop are obtained for the corresponding boundary conditions of the DPDHL with the inlet velocity of evaporator and condenser loop of $0.1 \mathrm{~m} / \mathrm{s}$, wall heat fluxes $20000 \mathrm{w} / \mathrm{m}^{\wedge} 2$ and the inlet condenser temperature of $283 \mathrm{k}$. The only varying parameter was the frequency of the reciprocating motion or in the other word, the frequency of the sinusoidal inlet velocity for the evaporation loop. The results of unsteady simulation indicate the following important points:

1. The frequency of the inlet velocity has a significant effect on the rate of heat removal from the evaporator. It can be concluded that the optimum loop time should be equal or more than:

$$
\begin{aligned}
& \qquad \Delta T=2 \times \Delta T_{\text {half }} \\
& \text { Where } \Delta T_{\text {half }}=\frac{0.5+0.005+0.125}{U_{\max }} .
\end{aligned}
$$

2. The numerical results also show that choosing the duration time less than the above value results in much higher temperature on the evaporator walls because the fluid could not transfer the heat from the evaporator to the condenser well.

3. In this study, a sinusoidal velocity profile is used, but there is no grantee that this profile is the best choice to obtain the maximum heat transfer rate. It is 


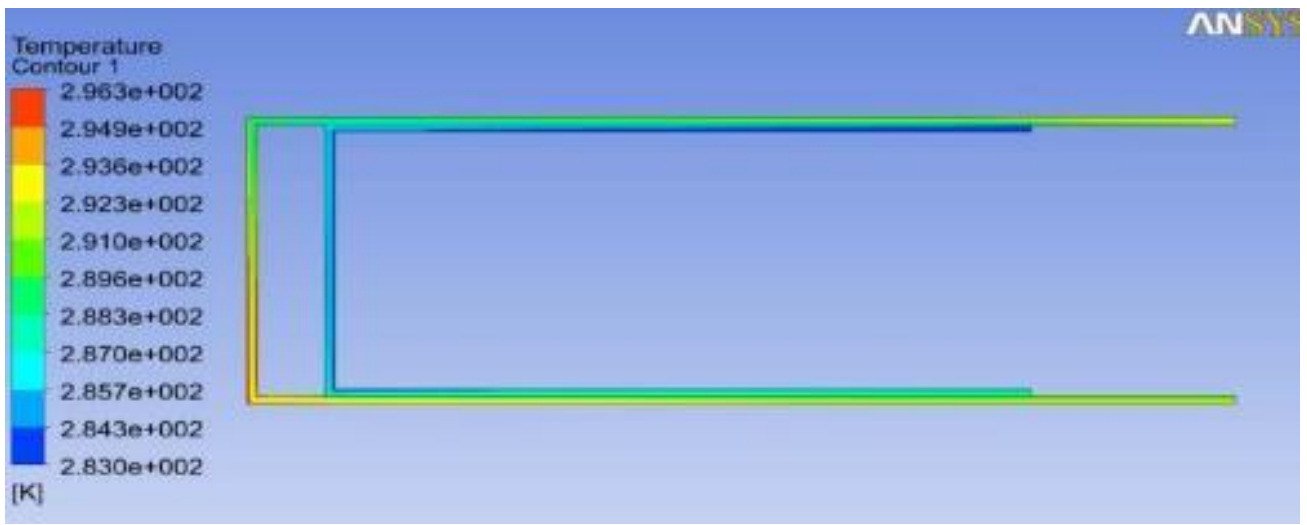

Figure 4. 16: Temperature contour for the evaporator, condenser and solid walls.

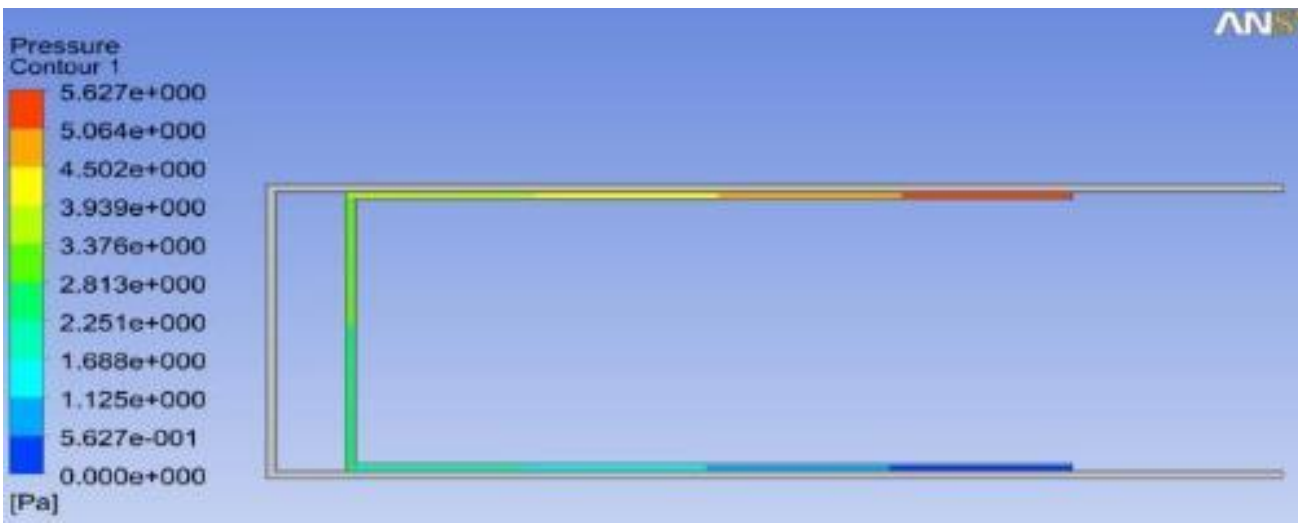

Figure 4. 17: Pressure contour for the condenser loop. 


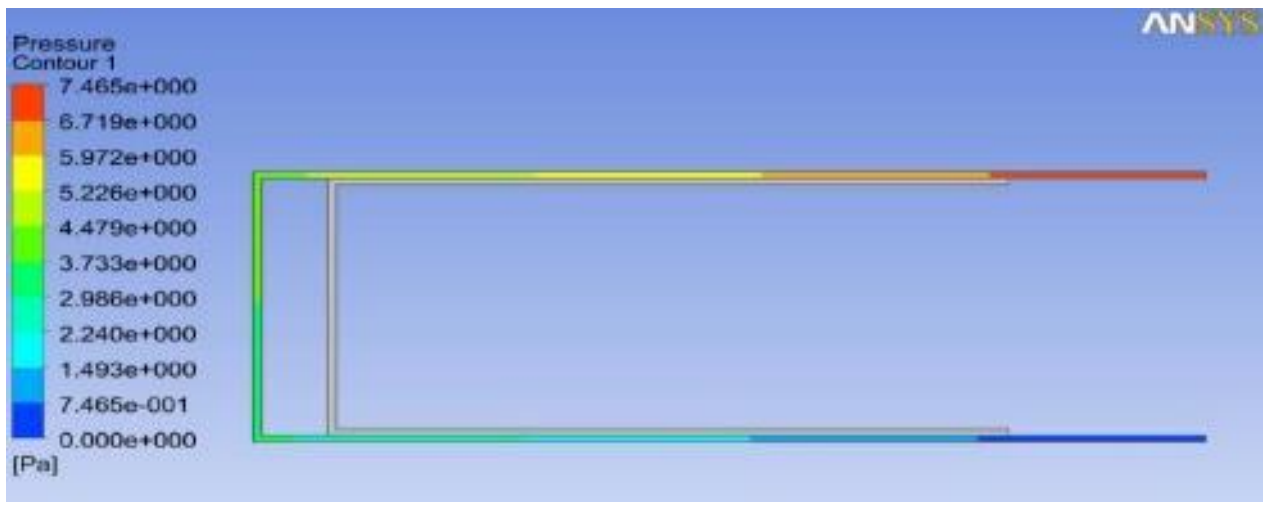

Figure 4. 18: Pressure contour for the evaporator loop.

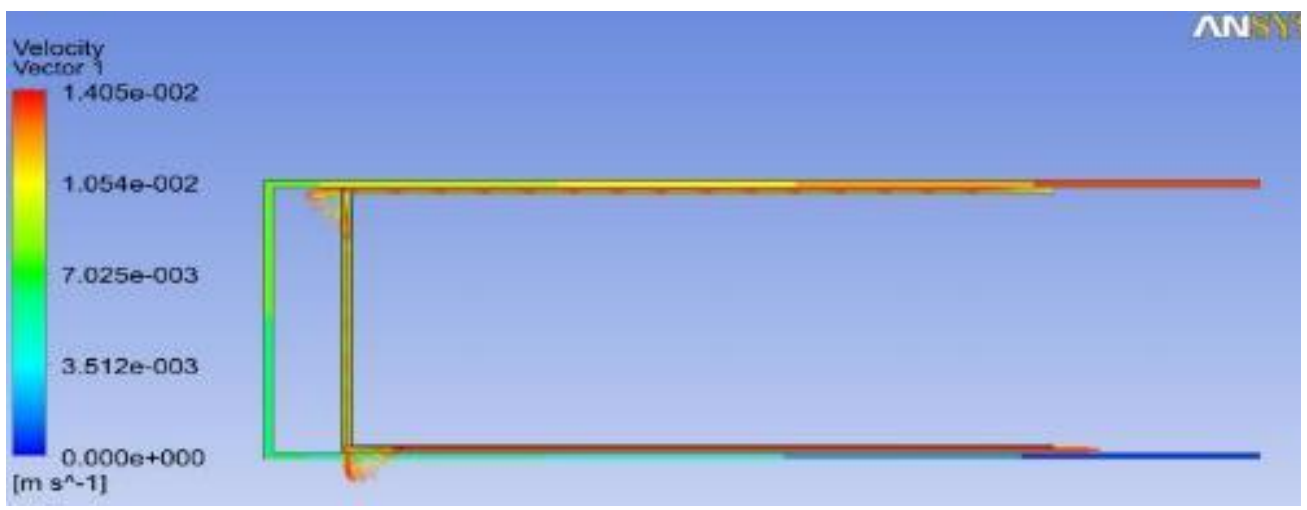

Figure 4. 19: Velocity vectors of the condenser loop. 


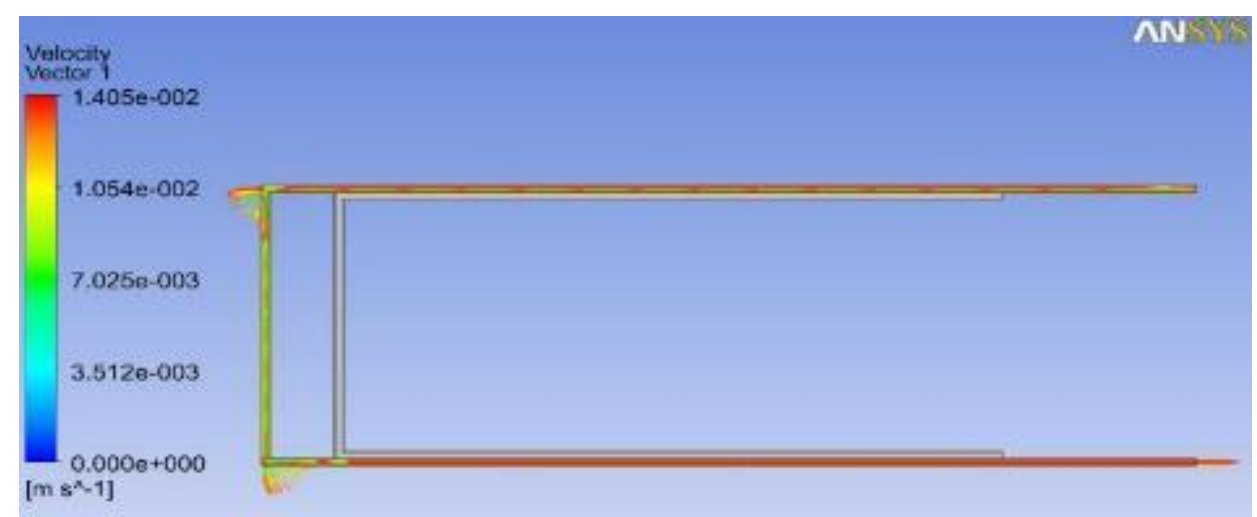

Figure 4. 20: Velocity vectors of the evaporator loop.
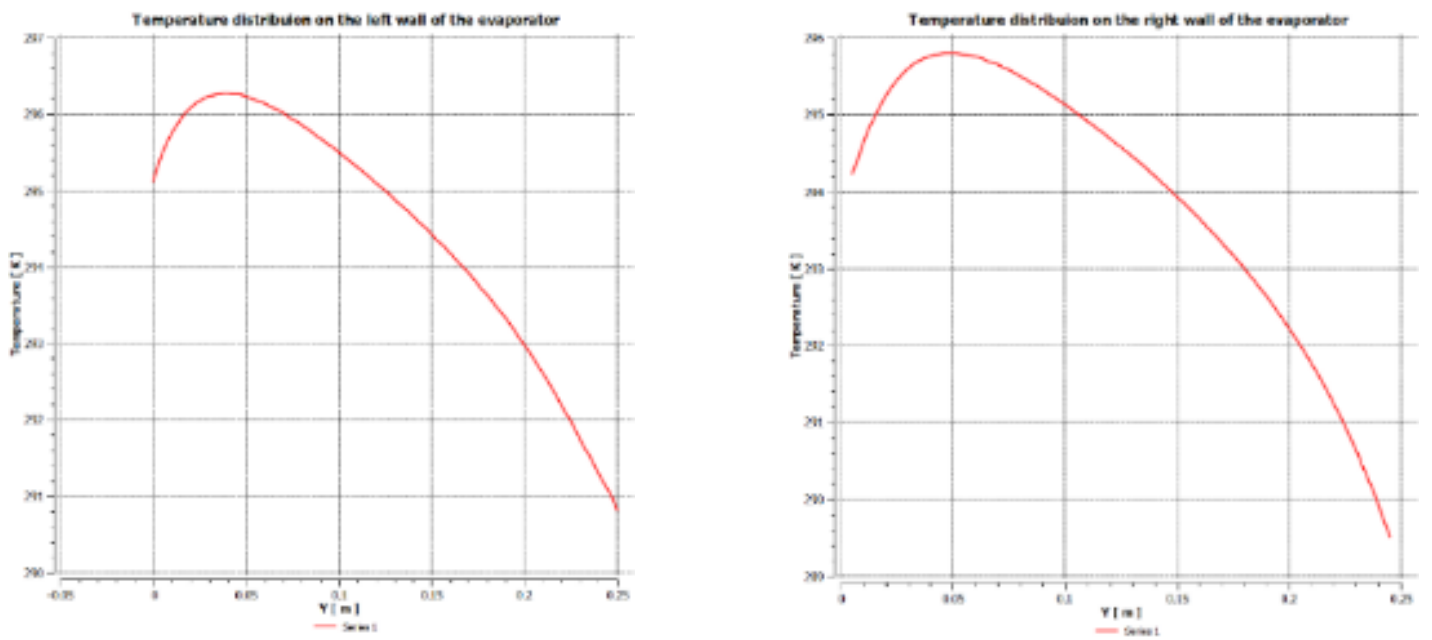

Figure 4. 21: Temperature profiles on the evaporator walls for $V_{\max }=0.01 \mathrm{~m} / \mathrm{s}$, $q^{\prime \prime}=2000 \mathrm{~W} / \mathrm{m}^{2}$ and $\mathrm{T}_{\text {condensor }}=283 \mathrm{~K}$. 

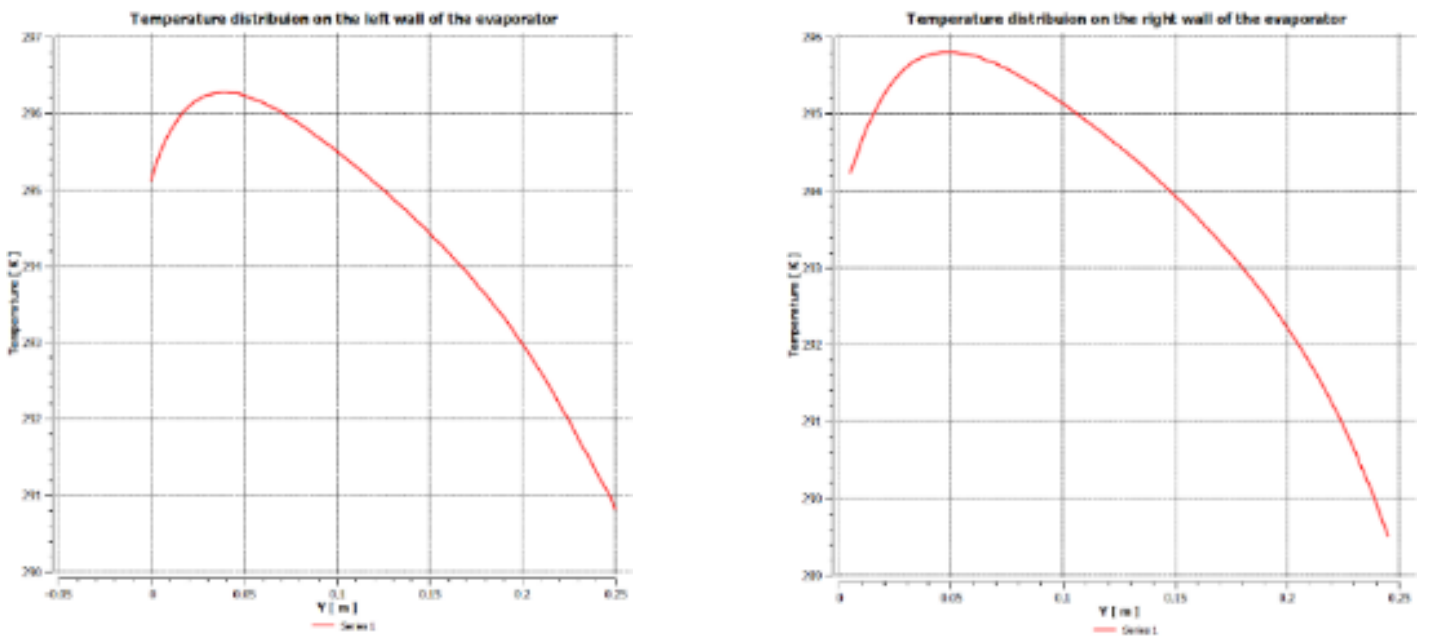

Figure 4. 22: Temperature profiles on the evaporator walls for $V_{\max }=0.01 \mathrm{~m} / \mathrm{s}$, $q^{\prime} "=5000 \mathrm{~W} / \mathrm{m}^{2}$ and $T_{\text {condensor }}=283 \mathrm{~K}$.
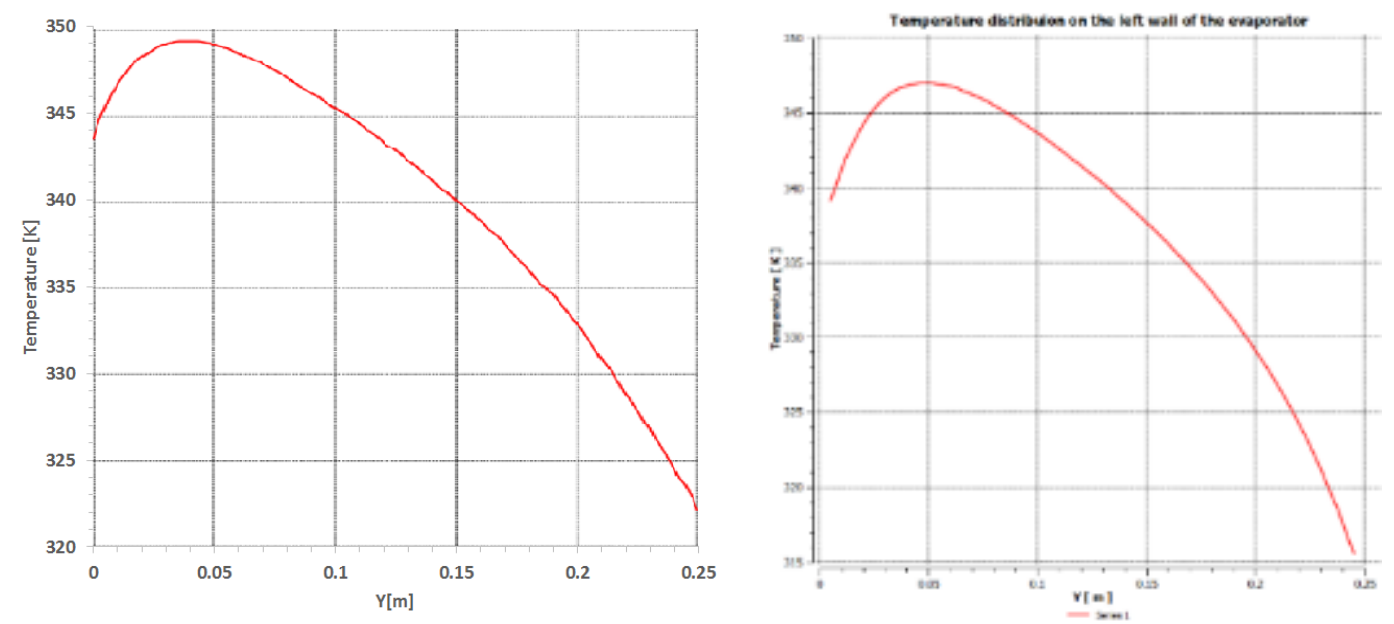

Figure 4. 23: temperature profiles on the evaporator walls for $V_{\max }=0.01 \mathrm{~m} / \mathrm{s}$, $q "=10000 \mathrm{~W} / \mathrm{m}^{2}$ and $\mathrm{T}_{\text {condensor }}=283 \mathrm{~K}$. 

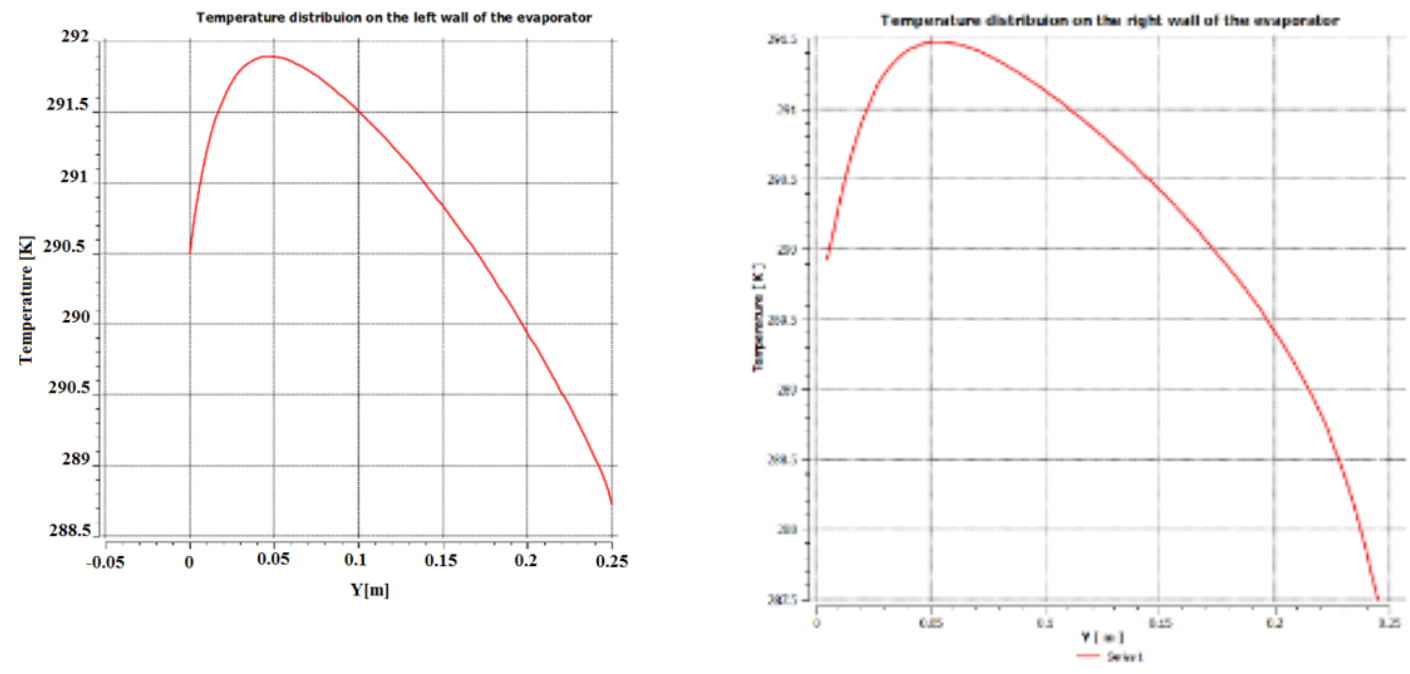

Figure 4. 24: temperature profiles on the evaporator walls for $V_{\max }=0.03 \mathrm{~m} / \mathrm{s}$, $\mathbf{q}^{\prime \prime}=2000 \mathrm{~W} / \mathrm{m}^{2}$ and $\mathrm{T}_{\text {condensor }}=283 \mathrm{~K}$.
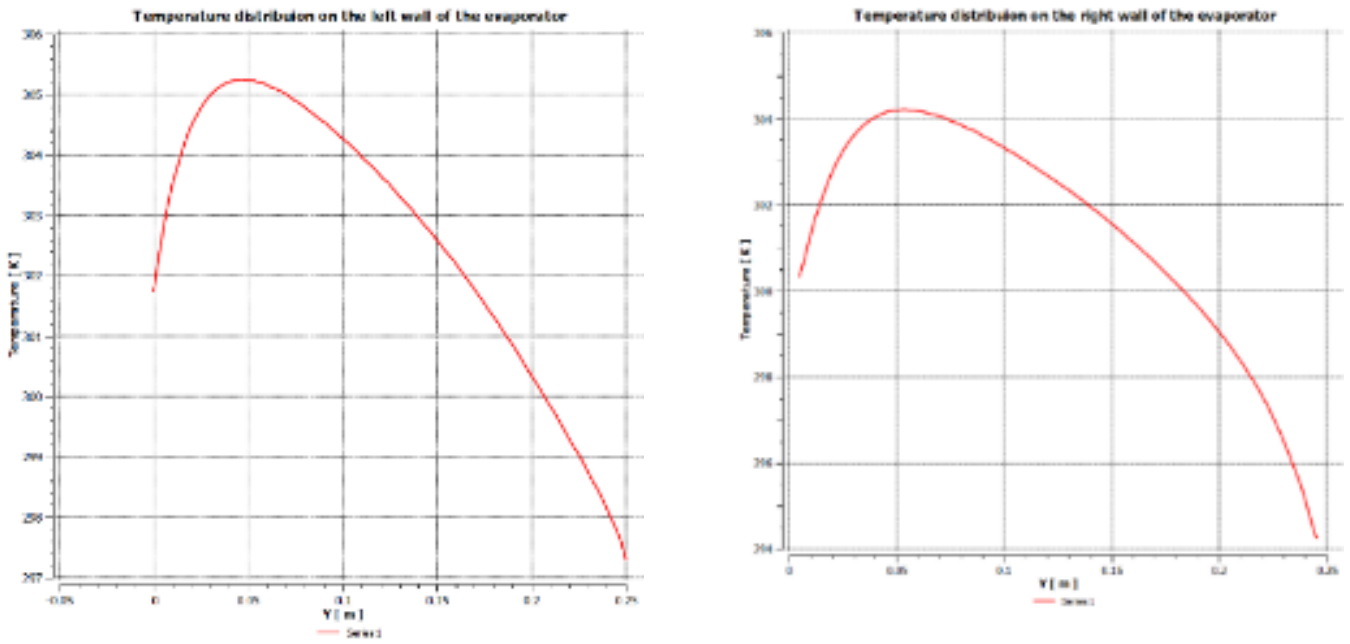

Figure 4. 25: temperature profiles on the evaporator walls for $V_{\max }=0.03 \mathrm{~m} / 2$, $\mathrm{q} "=5000 \mathrm{~W} / \mathrm{m}^{2}$ and $\mathrm{T}_{\text {condensor }}=283 \mathrm{~K}$ 


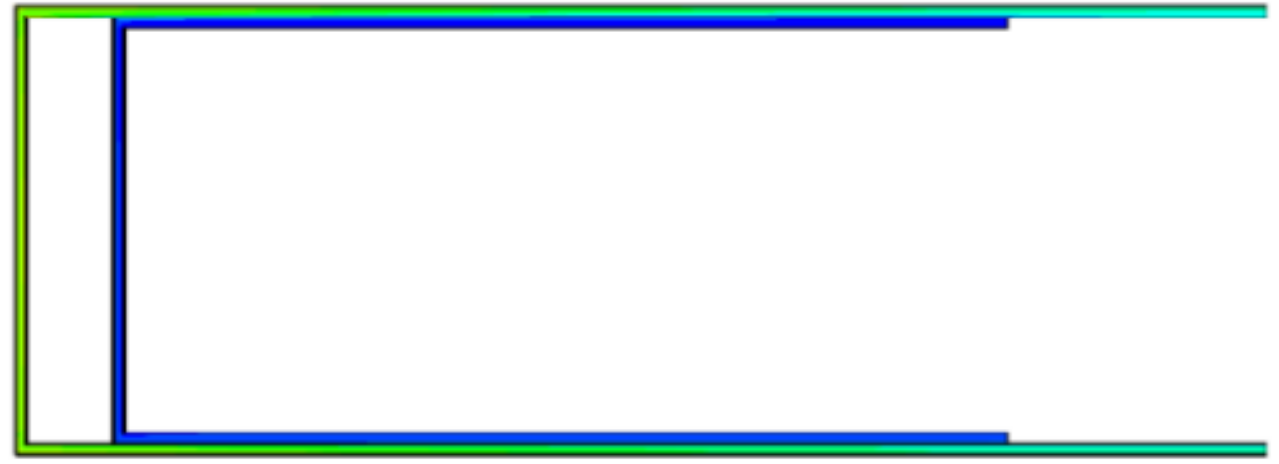

Figure 4. 26: Temperature contour on the computational domain

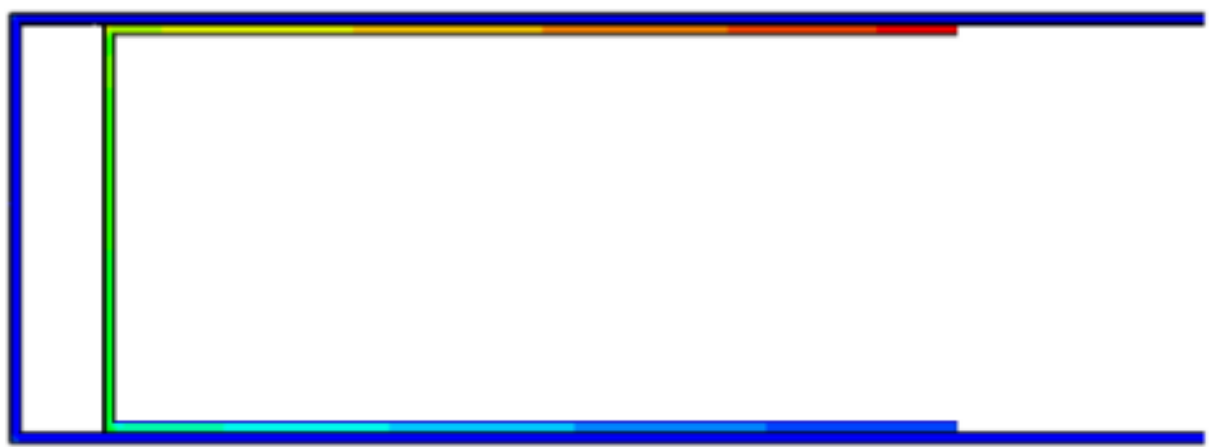

Figure 4. 27: Pressure contour on the computational domain. 


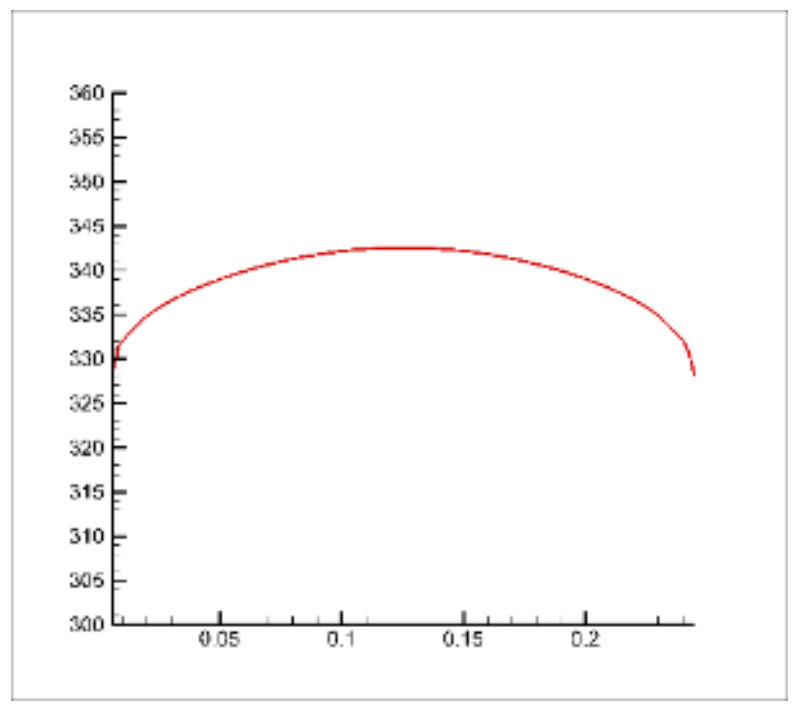

Figure 4. 28: Temperature profile on the evaporator surface, $t=15 s, q " '=20000$ $\mathbf{W} / \mathbf{m}^{2}$.

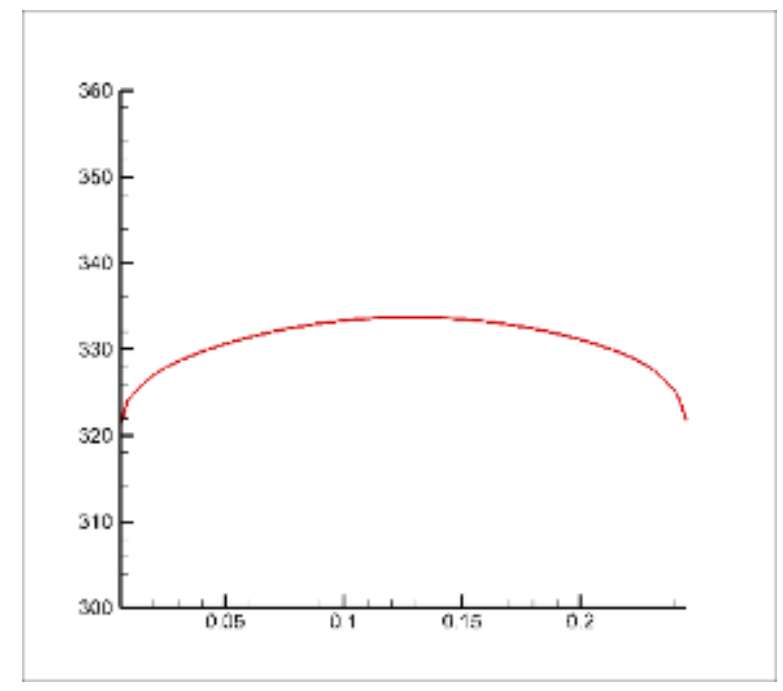

Figure 4. 29: Temperature profile on the evaporator surface, $T=20 \mathrm{~s}, \mathrm{q}$ ' $=$ $20000 \mathrm{~W} / \mathrm{m}^{2}$ 


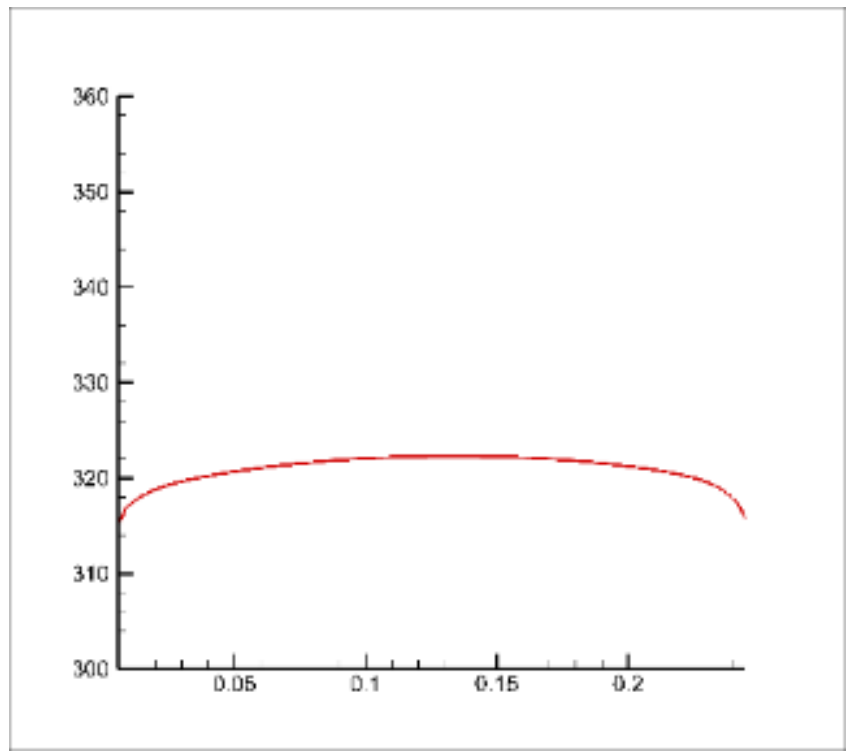

Figure 4. 30: Temperature profile on the evaporator surface, $T=30 \mathrm{~s}, \mathrm{q}, "=20000$ $\mathrm{W} / \mathrm{m} 2$. 
believed that a velocity profile with longer residence time and shorter circulation time will have a better performance for the RMDHLs.

4. In this study, a reciprocating velocity profile has been applied to the inlet boundary, and outlet boundary is set to constant pressure boundary.

Figures 4.27 and 4.28 show the averaged pressure and temperature contours on the computational domain for a reciprocating loop. Contrary to the DPDHL, the temperature contour is in symmetry for the RMDHL system. Figures $4.30-4.31$ show the temperature profile on the wall of the evaporator for different heating periods of the reciprocating loop. As can be seen, the temperature profile is more uniform for the RMDHL, in which the maximum temperature occurs at the midpoint of the walls.

\subsection{Investigation into the Effect of various turbulence models on the accuracy of Numerical Model.}

The purpose of this study is to determine the most suitable turbulence model that produces the closest approximation to equilibrium conditions on the cold plate of the RMDHL. Turbulence models can be classified as Zero, One, Two or Stress equation models based on the number of partial differential equations solved in addition to the mean flow equation (Nallasamy 1987). The $k-\varepsilon, k-\quad$ and the $k-\omega$ models belong to the two-equation models. The standard and modified forms of the $k-\varepsilon$ model is used in a majority of flows reported in the literature. While the equally popular Transition $k$ -

- $\omega$ and Transition SST are three equation models. This study compared the accuracy of the predictions of the Standard k- $\varepsilon$ Model, Renormalization-group (RNG) k- $\varepsilon$ 
Model, Realizable k- $\varepsilon$ Model, Standard k- $\omega$, Shear-Stress Transport (SST) k- $\omega$ Model and Transition SST Model.

The formulation of the conjugate heat transfer in the present study is based on the three-dimensional single-phase equations of continuity, momentum, and energy under the conditions of incompressible fluid, negligible dissipation and pressure work, and constant fluid properties. The current analysis is an open loop configuration for the reciprocating loop. The open loop configuration is achieved using a virtual loop. The loop is assuming adiabatic, isochoric and isobaric conditions. In the forward direction the boundary conditions for the inlet remains a velocity inlet, and in the reverse flow, the boundary condition reverses to a velocity outlet. The Virtual loop was successfully implemented to reduce computational costs associated with fluid-structure interaction required in modeling the pump.

The mesh information for numerical investigation is shown in Fig 4.12. Numerical values for the boundary conditions are provided in Table 4.2. For all numerical studies, grid independence studies are always necessary to establish computational accuracy and were equally performed in this work. A time-step of 0.005 was adopted for the study and convergence was achieved after an average of sixty thousand iterations, with an average total flow time of 300s. The mesh independence studies were conducted for two more Grids with coarse and fine meshes settings in ANSYS Fluent. With respect to temperature variation on the plate surface, the variation of the results between the coarsest and finest meshes was $2 \%$ while the results showed less than $1.5 \%$ change in the last two grid size. The criteria for convergence was set at 
$10^{-4}$ for the continuity, $x, y$ and $z$ momentum, turbulent kinetic energy and turbulence dissipation rate, while a convergence criterion of energy was set at $10^{-7}$.

The flow channel for the experimental and numerical setups of this conjugate heat transfer study consists of rectangular channels at the heat addition site within the cold plate and circular channel in the connecting piping of the condenser section where heat removal occurs. The focus of the study is the characteristics of the convection and conduction in the rectangular channel with constant heat flux. The results of this analysis are compared with similar works.

For the present high heat flux application, a two-phase RMDHL is considered. The commercial ANSYS Fluent CFD code was employed to simulate the RMDHL numerically. The first step in the model development is the generation of a 3D CAD model of by SolidWorks. Both the condenser and evaporator of the loops are enclosed with a solid wall. The solid walls are copper, and thermophysical properties for them are obtained from the fluent database. The working liquid in the RMDHL loops is water. To simplify the numerical modeling and understand the reciprocating flow heat removal characteristics, an open loop configuration of the heat loop is modeled. The model includes the loop condenser with specified inlet and outlet conditions associated with the condenser.

Temperature variation on the cold plate for all the solutions is typical of the results of single phase RMDHL cooling systems (O. Popoola, Soleimanikutanaei, and Cao 2016b)(O. T. Popoola and Cao 2015). The temperature uniformity across the cold plate is such that the temperature is lower at the edges and gradually increases through 
the middle. This variation is different from conventional cooling using dynamic pumping system where the temperature will progressively increase from the coolant inlet to the outlet. Based on a time step of $0.005 \mathrm{~s}$ adopted for the current study, the results of the numerical analysis indicate that the temperature scatter among the numerical models tested is about $2 \mathrm{~K}$. The temperature range across the cold plate is within $3^{\circ} \mathrm{C} . k$ - $\varepsilon$ models however produces a higher temperature uniformity across the cold plate than those of the $k$ - $\omega$ based models. The comparison of the results of the numerical model to the data collected experimentally was made both graphically and statistically.

Additional visual comparison for the results of the turbulence based numerical models was carried using a consistent post-processing setting, as shown in Fig. 4.31. The figures show the temperature gradients as expected in such numerical analysis, which is that heat enters the system from the cold plate (evaporator) and the heat is removed from the system at the condenser, regarding the contour of varying temperature across evaporator 


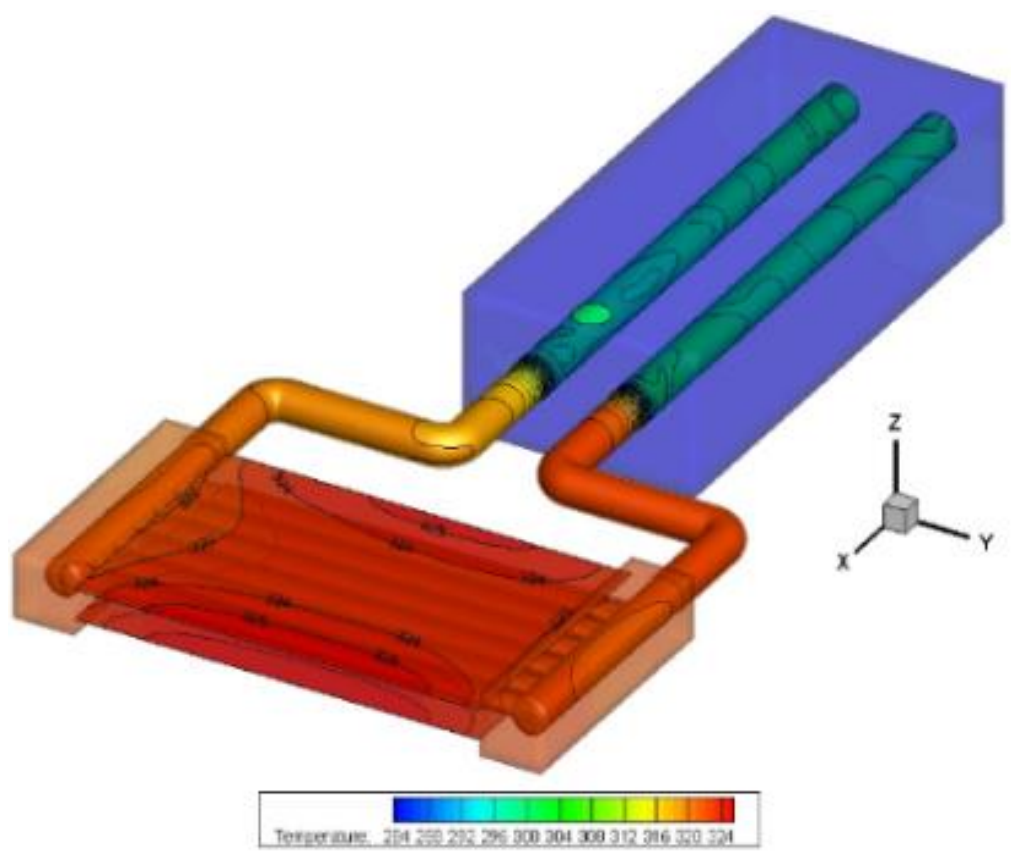

(a) Standard $k-\varepsilon$ Model

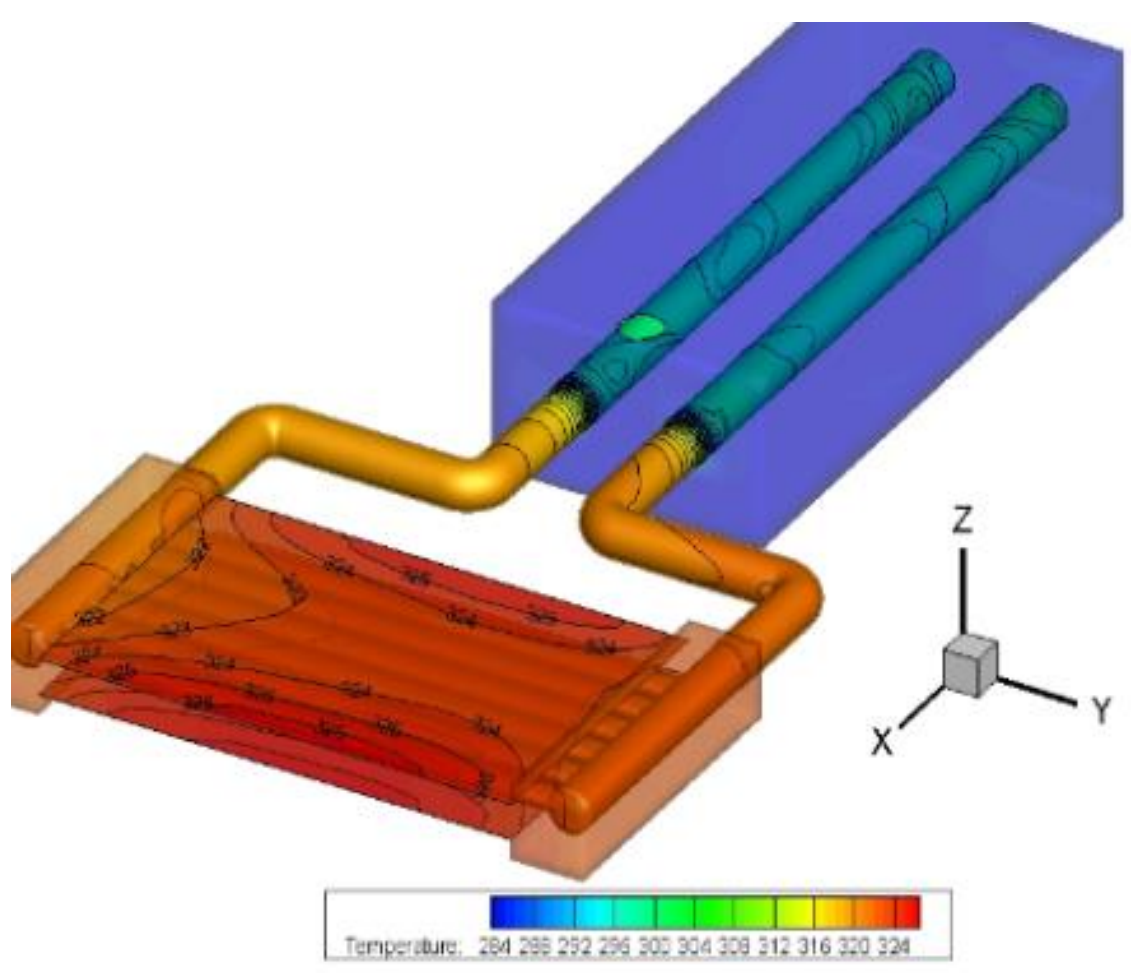

(b) Realizable $k$ - $\varepsilon$ Model 

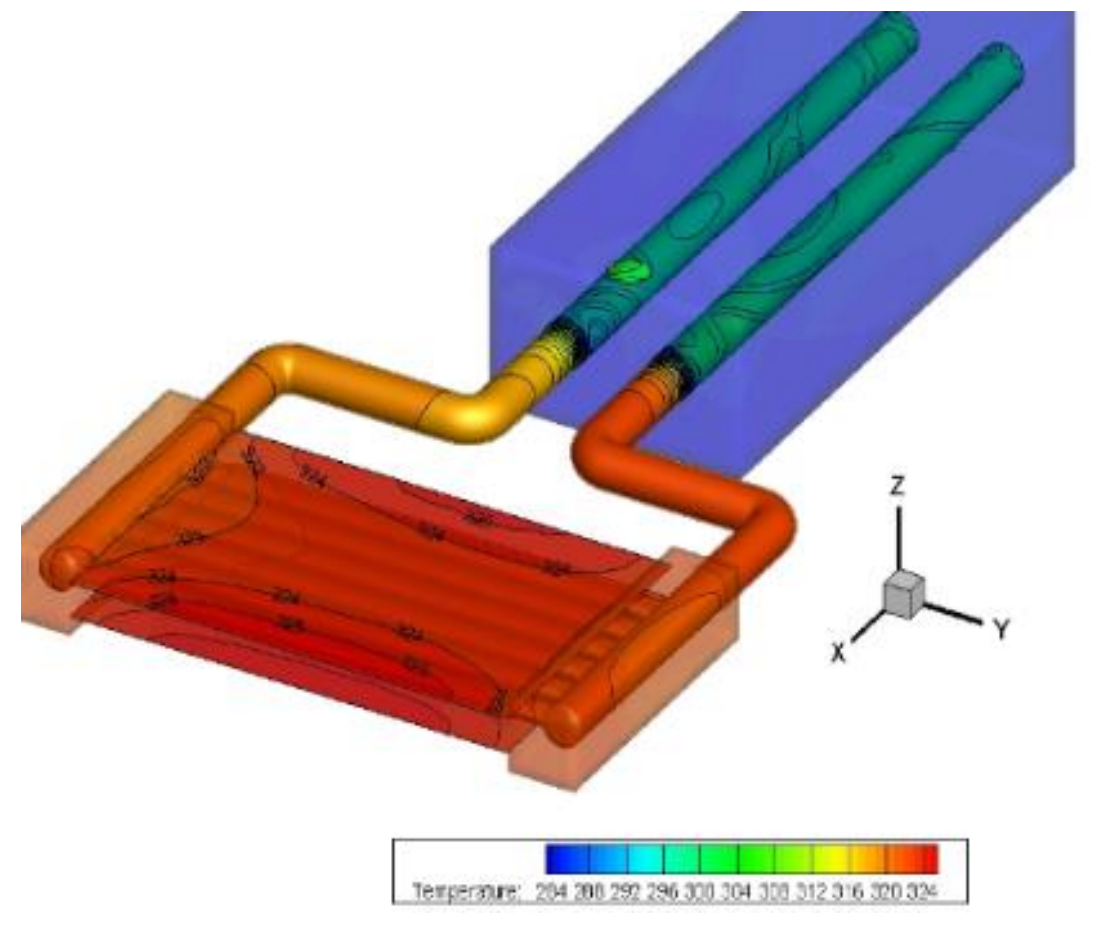

(c) RNG $k$ - $\varepsilon$ Model

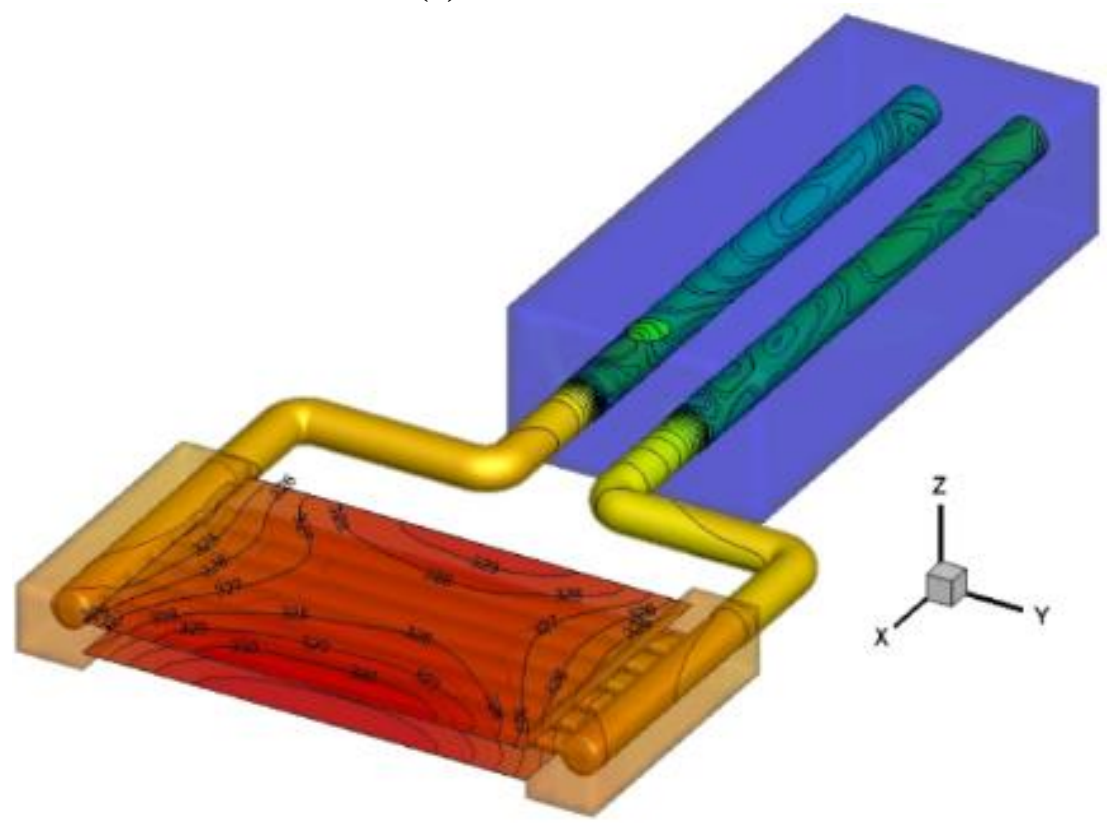

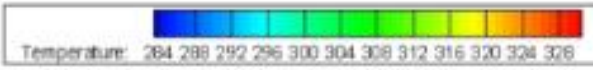

(d) SST $k$ - $\omega$ Model 


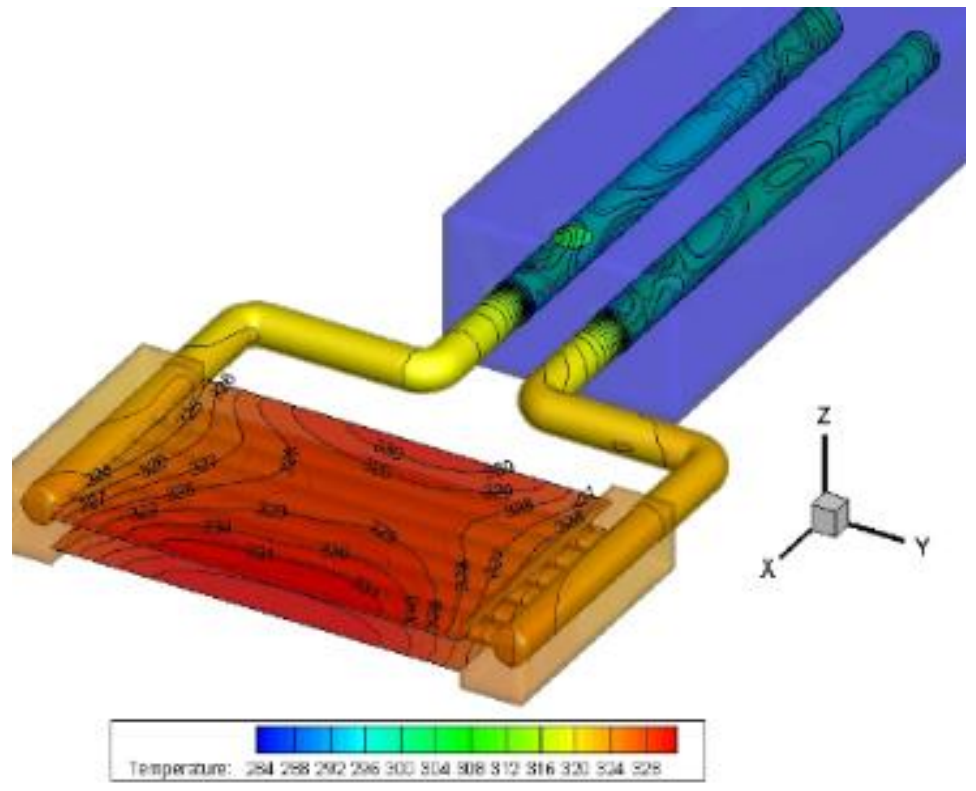

(e) Standard $k$ - $\omega$

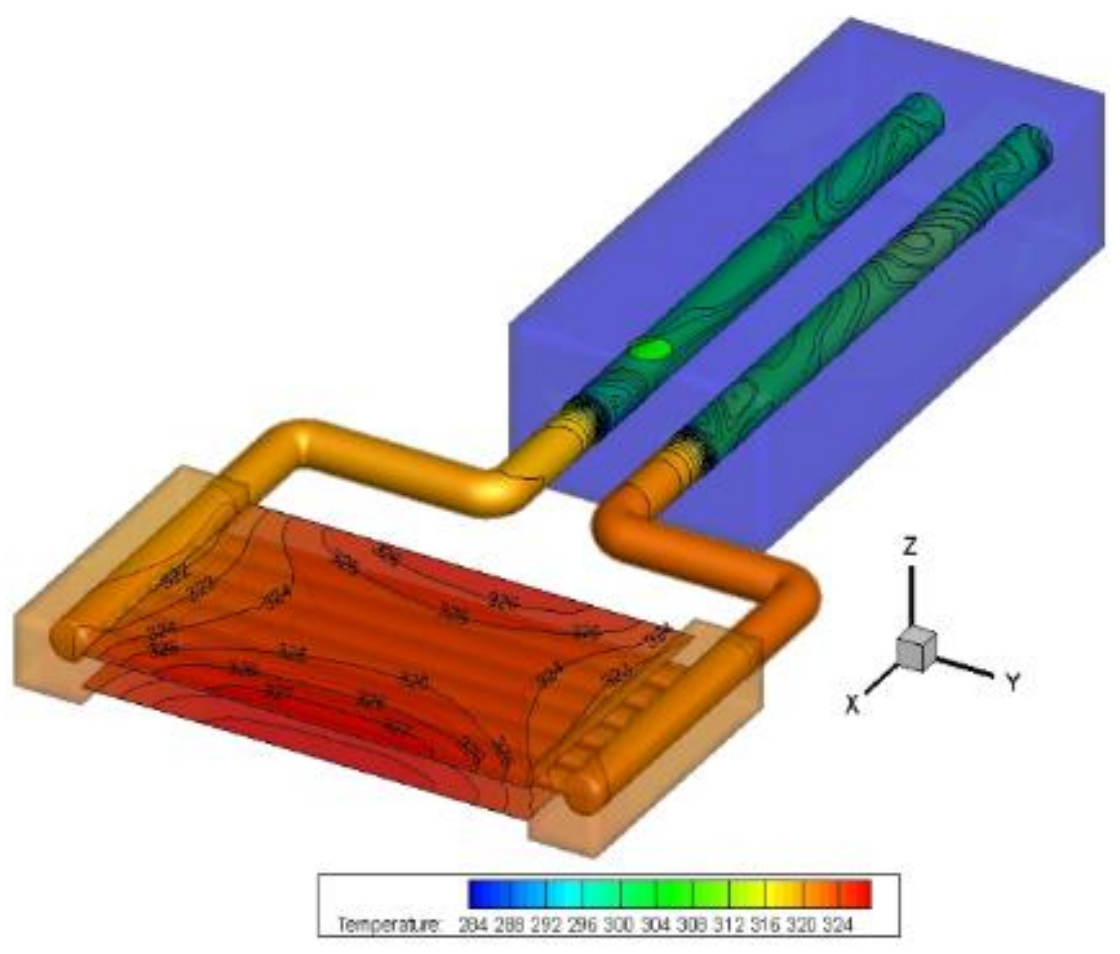

(f) Transition $k-\quad-\omega$ 


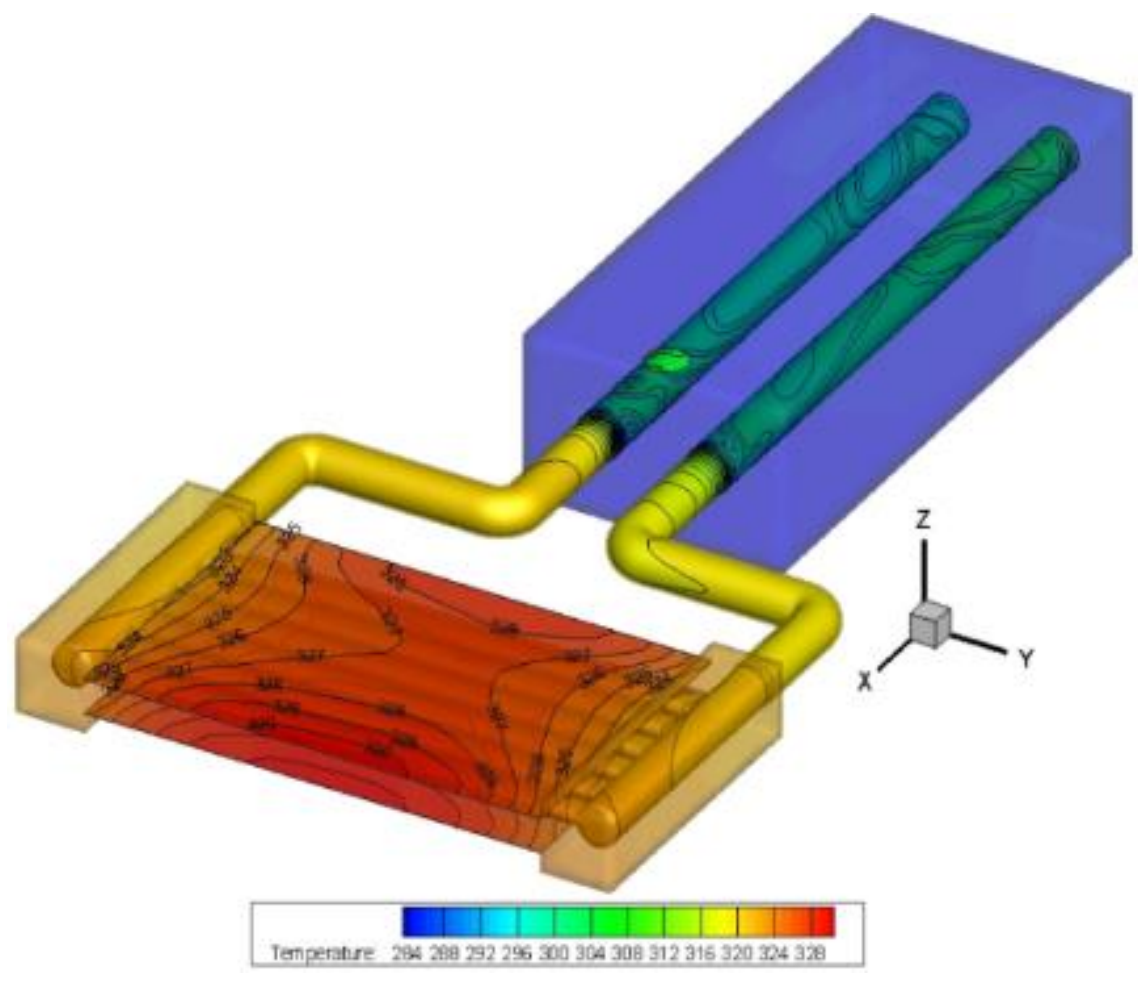

(g) Transition SST

Figure 4. 31: Temperature contours of RMDHL assembly model with various turbulence prediction models. 
for varying heat transfer rate at different stages in the numerical simulation. From Fig. 4.31 it was evident that the results of the numerical models vary significantly for the cold plate (evaporator), condenser and the cooling fluid in the RMDHL loop. Fig. 4.32 is an $\mathrm{x}$-y plot of the numerical results from different turbulence models as compared with the corresponding temperature measurement at each thermocouple location. From the results it can be seen that some models such as RNG k- $\varepsilon$ and Standard k- $\varepsilon$ match the experimental data much better than others.

To standardize the results of this work and provide an empirical basis for the results of this study to be benchmarked against other works, a numerical model calibration template (Moriasi et al. 2007) is adapted for comparing and ranking numerical model predictions for the cold plate temperature with measured data. The template investigates the accuracy of each numerical prediction and then benchmarks each numerical result against data. A summary of the statistical analysis is presented in Table 4.8. From the template, all the models appropriately reproduce the magnitudes of measured data.

Regarding computational time, the average number of iterations for convergence for all the turbulence models averaged about $60 \mathrm{k}$ iterations. However, the computational time for the standard $k-\varepsilon$ and standard $k-\omega$ models were significantly lower while the Transition $k-\quad-\omega$ was significantly higher than the other Models. Although this work did not investigate the 


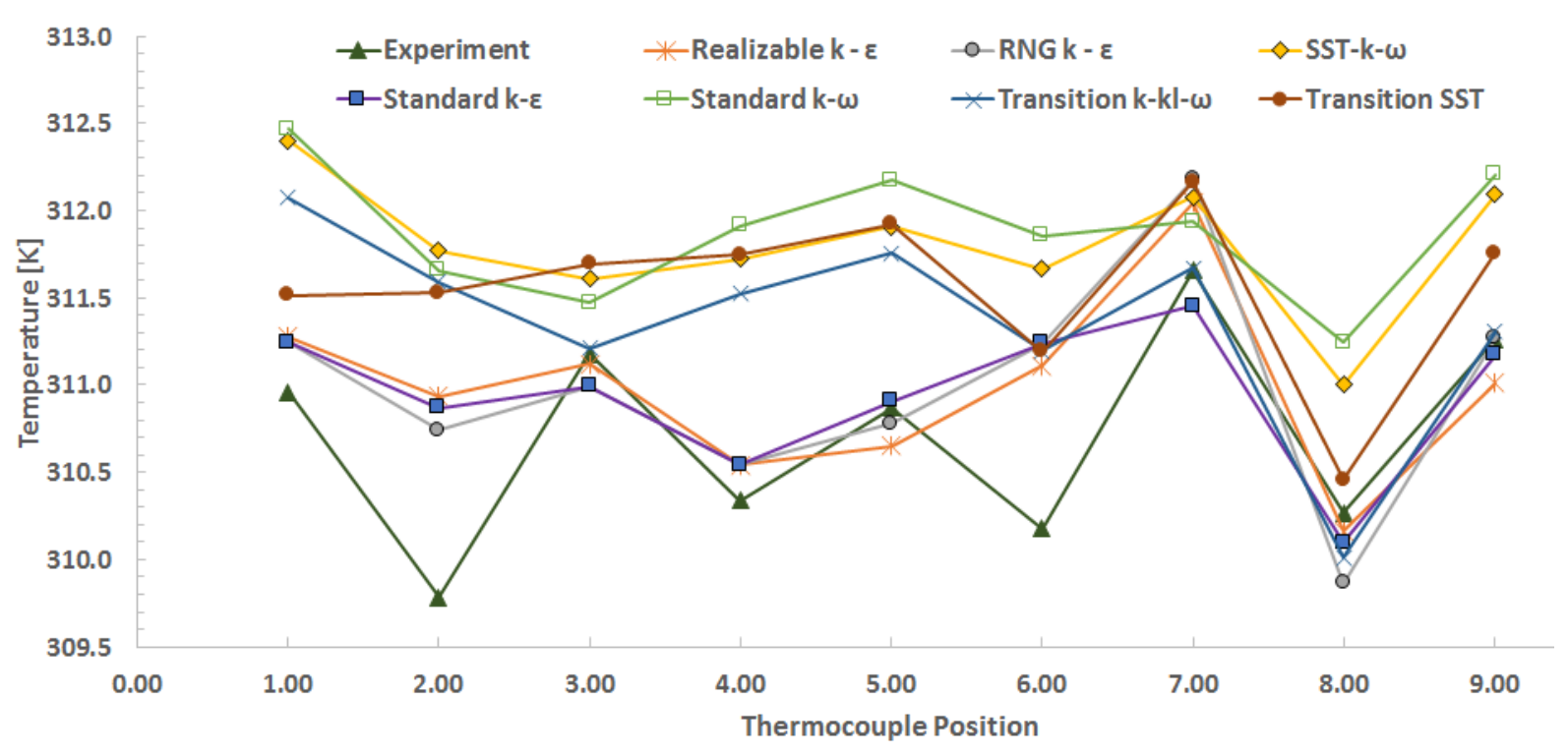

(a)

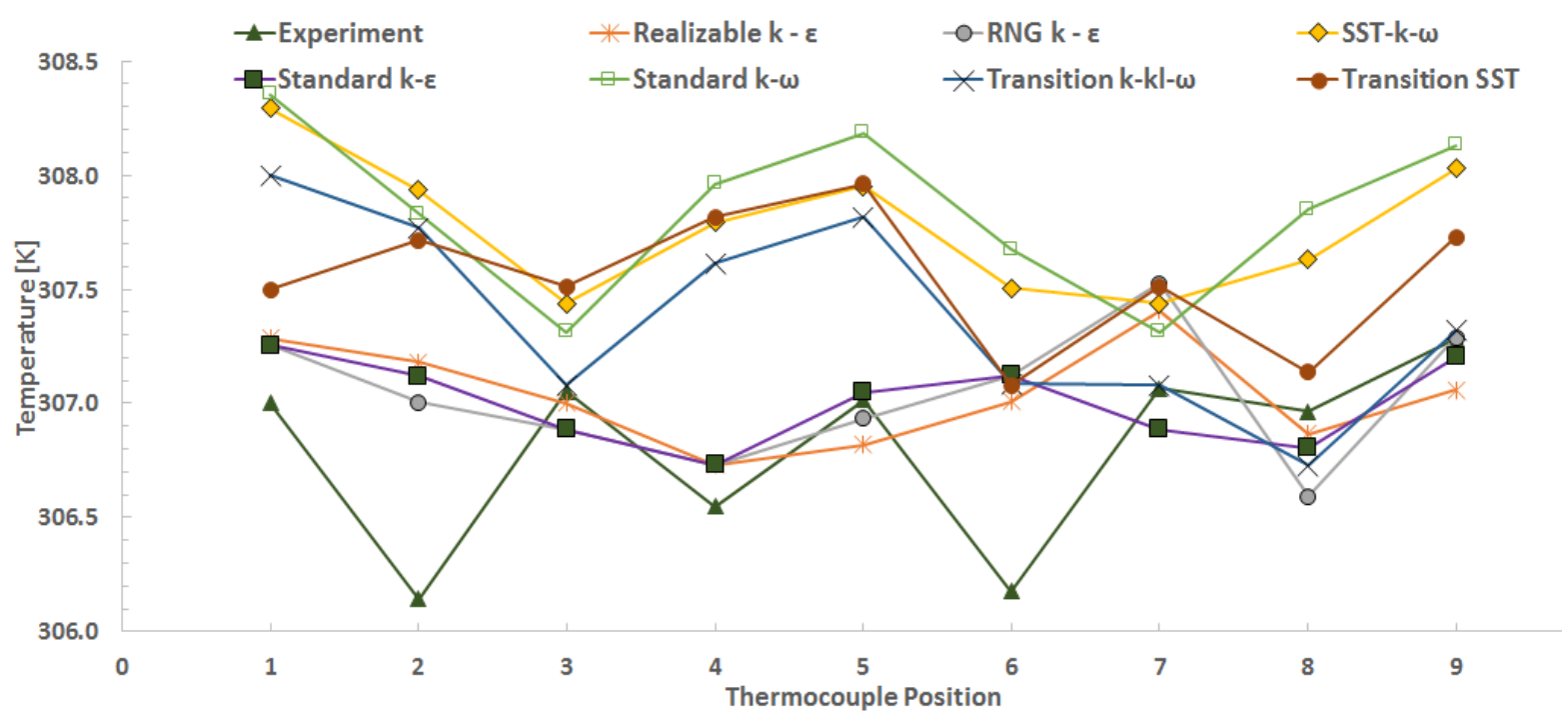

(b)

Figure 4. 32. Temperature distributions over the cold plate for experimental and the numerical models for (a) $8.8 \mathrm{KW} / \mathrm{m}^{2}$ (b) $6.7 \mathrm{KW} / \mathrm{m}^{2}$ 


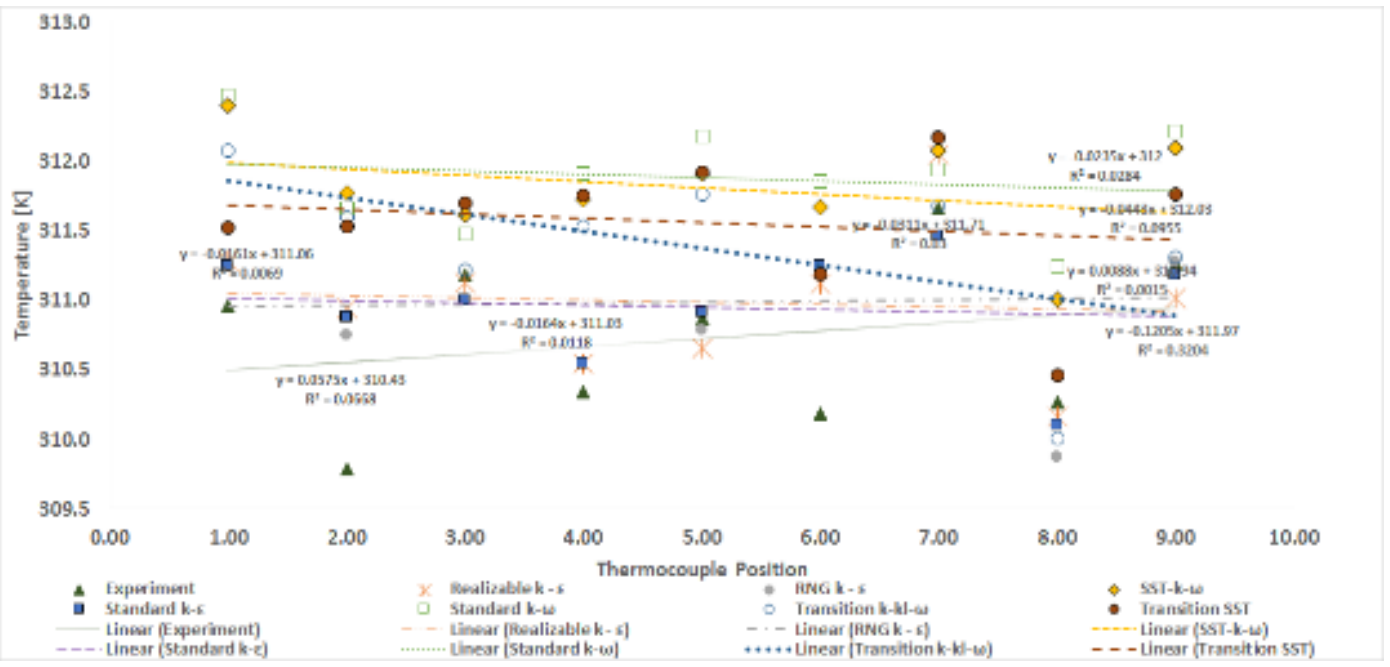

Figure 4. 33: The $y$-intercept of the best-fit regression temperature distributions over the cold plate 
Table 4. 2: Results for statistical and ranking for turbulent prediction methods

\begin{tabular}{|l|l|c|c|c|c|c|c|c|c|}
\hline \multirow{2}{*}{ Model } & \multirow{2}{*}{ Experiment } & $\begin{array}{l}\text { Realizable } \\
k-\varepsilon\end{array}$ & $\begin{array}{l}\text { RNG } \\
k-\varepsilon\end{array}$ & $\begin{array}{l}\text { Standard } \\
k-\varepsilon\end{array}$ & $\begin{array}{l}\text { SST- } \\
k-\omega\end{array}$ & $\begin{array}{l}\text { Standard } \\
k-\omega\end{array}$ & $\begin{array}{l}\text { Transition } \\
k-\end{array}-\omega$ & $\begin{array}{l}\text { Transition } \\
\text { SST }\end{array}$ \\
\hline \multirow{3}{*}{ Regression } & $\mathrm{r}$ & & 0.62 & 0.67 & 0.56 & 0.51 & 0.39 & 0.30 & 0.60 \\
\cline { 2 - 10 } & $\mathrm{R}^{2}$ & 0.067 & 0.007 & 0.001 & 0.019 & 0.096 & 0.028 & 0.320 & 0.030 \\
\cline { 2 - 10 } & Slope & 0.058 & -0.016 & 0.009 & -0.016 & -0.044 & -0.002 & -0.121 & -0.031 \\
\cline { 2 - 10 } & Intercept & 310.43 & 311.06 & 310.94 & 311.03 & 312.03 & 312.00 & 311.97 & 311.71 \\
\hline NSE & & 0.12 & 0.11 & 0.15 & -3.31 & -3.97 & -1.63 & -1.78 \\
\hline PBIAS & & -0.08 & -0.08 & -0.07 & -0.35 & -0.37 & -0.21 & -0.27 \\
\hline RSR & & 0.94 & 0.94 & 0.92 & 2.08 & 2.23 & 1.62 & 1.67 \\
\hline
\end{tabular}


effect of turbulence models on pressure drop, the value of the slope of the RNG $k-\varepsilon$ may indicate that the pressure drop may be influenced by the turbulence models. The value of the slope for the RNG $k-\varepsilon$ model has a positive value whereas all the other models have a negative value. Incidentally, the slope of the experimental results also has a positive value.

The value of the slope for all turbulence models as well as the experimental data is within the range of \pm 1 . The slope indicates that the results from all the turbulence models and the measured experimental values are linearly related. From the y-intercept and the slope values, the data suggests that the RNG $k-\varepsilon$ provides the best prediction for the magnitude of the average temperature of the measured data. The Slope indicates that the RNG $k-\varepsilon$ also provides a better prediction of the linear variation in temperature across the cold plate surface. NSE, RSR, and PBIAS for all the numerical models were less than \pm 1 , implying that all the turbulence models will provide suitable correlations to the experimental results. This outcome is of importance to address the point raised in the introductions that before this study correlation for oscillating flow is not explicitly developed as they are obtained from using correlations relating to unidirectional (continuous) flow.

The PBIAS shows that the magnitude of the results for the numerical $k$ - $\omega$ based models tends to be larger than the magnitude of the experimental data. Physical examination of the plots indicates that the $k$ - $\omega$ models may provide a better prediction of the fluctuations in temperature. However, the RSR values suggest that the $k-\omega$ Models contain more noise related values than the $\mathrm{k}-\varepsilon$ Model. The reason for this may 
be due to the fact that the $k$ - $\omega$ Models contain a wall function as part of its formulation, and the sinusoidal variation of flow velocity and pressure may make the results of the $k$ - $\omega$ Models less stable when compared to the results of the $k$ - $\varepsilon$ models. On the other hand, NSE indicates how well the plot of observed data fits the simulated data (Moriasi et al. 2007). We can conclude that the RNG $k$ - $\varepsilon$ based models provide better magnitude predictions, collinearity to the measured data, and least noise. The standard $k$ - $\varepsilon$ model and the realizable k- $\varepsilon$ Model are just as efficient as standard $k-\varepsilon$ models but the $k$ - $\omega$ based models were the least accurate in magnitude prediction.

\subsection{D Single - Phase Modeling}

This study aims to numerically investigate the possibility of applying the RMDHL to possible liquid cooling systems and compare its performance to that of a dynamic pump driven heat loop (DPDHL) in terms of a continuous or unidirectional flow cooling system. The second objective of the present study is to validate the numerical investigation with the experimental research and compare the performance of the RMDHL with the conventional continuous cooling loops in terms of the temperature level, temperature uniformity and heat removal rate from the surface of the heat source.

The commercial ANSYS Fluent CFD code was employed to simulate both the DPDHL and RMDHL numerically. The first step in the model development is the generation of a 3D CAD model of by SolidWorks. Both the condenser and evaporator of the loops are enclosed with a solid wall. The solid walls are aluminum, and 
thermophysical properties for them are obtained from the fluent database. The working liquid in both the RMDHL and DPDHL loops is water.

The formulation in the present study is based on the single three-dimensional phase turbulent flow equations of continuity, momentum, and energy under the conditions of incompressible fluid, negligible dissipation and pressure work, constant fluid properties, and the Boussinesq approximation, along with appropriate boundary conditions. However, the specifications of the inlet conditions to the condenser and the outlet conditions from the condenser for both DPDHL and RMDHL to achieve a relevant equivalency for comparison may be a bit challenging.

For performance comparison, the goal of this investigation is accurately select a flow velocity for the continuous flow which is equivalent to the reciprocating flow velocity. In doing this, it is essential to note that there are several fundamental differences between the reciprocating flow and the continuous flow. First, the velocity profiles are entirely different. Whereas the maximum axial velocity for the continuous flow at the center of the channel is of so-called parabolic effect, the maximum axial velocity for a fast oscillating flow occurs close to the wall, resulting in the so-called annular effect (T. S. Zhao and Cheng 1998b). Also, the transition from laminar to turbulence in reciprocating flow is different for that of the continuous flow. Even though the categorization of reciprocating flow regime as either laminar or turbulent is based on the Reynolds number, the definition of the critical Reynolds number for the reciprocating flow in the main pipe is given by:

$$
R e_{\omega}=\frac{\omega D^{2}}{v}=354.32
$$


while the Reynolds number for the reciprocating flow in the rectangular channels for the cold plate is determined as:

$$
\left(R e_{\omega}\right)_{x}=\frac{\omega x_{\max }^{2}}{v}
$$

where $x_{\max }$ represents the maximum displacement of the fluid through the assumption that the fluid moves as a plug flow. In this case, it is equal to the minimum theoretical length of the channel. This value of the Reynolds number in the pipe indicates that the flow in the pipe is turbulent. Since the hydraulic diameter of the cold plate is smaller than the diameter of the pipe, it follows that the flow in the rectangular channels in the cold plate is turbulent.

The second fundamental parameters dictating the effect of the oscillating fluid flow frequency (Menon, Weber, and Chang 1984) is the Womersley number $(\alpha)$ and the depth of penetration of the flow $(\delta)$. At low $\alpha$, the flow is quasi-steady, i.e., the fluid particles everywhere respond instantaneously to the applied pressure gradient. When $\alpha$ is large as in the case of this study case, the motion of the boundary layer follows the pressure gradient more closely than the laminate of the fluid in the tube core, which shows phase lags to the imposed pressure gradient. Based on the fluid flow in the given cold plate of this simulation, the two terms are defined as follows:

$$
\begin{aligned}
& \begin{array}{l}
\alpha=\frac{D}{2} \sqrt{\frac{\omega}{v}} \\
\omega=2 \pi n
\end{array}=0.480 \frac{\mathrm{rad}}{\mathrm{s}} \\
& \text { where } D=\text { hydraulic radius }=0.020066 \mathrm{~m}
\end{aligned}
$$


Table 4. 3: Boundary conditions for the resent simulation.

\begin{tabular}{lll}
\hline Boundary conditions & \multicolumn{2}{l}{ Values and their range } \\
\hline $\begin{array}{l}\text { Inlet velocity of the } \\
\text { evaporator }\end{array}$ & $\begin{array}{l}\text { varying } \\
\text { stroke }(\mathrm{cm}) \\
\text { frequency } \\
(\mathrm{n})\end{array}$ & 7.62, \\
Inlet velocity of the & $1.223 \mathrm{~m} / \mathrm{s}$ & \\
condenser & & \\
Inlet temperature of the & $283 \mathrm{~K}$ & \\
condenser & $0.0001 \mathrm{~m}$ & \\
Solid wall thickness & Heat $(\mathrm{W})$ & Average Heat Flux $\left(\mathrm{W} / \mathrm{m}^{2}\right)$ \\
Heat (Q) & 606.00 & $8,780.16$ \\
& 558.00 & $8,084.70$ \\
& 505.00 & $7,316.80$ \\
& 464.00 & $6,722.77$ \\
\hline
\end{tabular}

Table 4.4: Dimensions, geometries and boundary conditions for the present cooling loop simulation.

\begin{tabular}{lll}
\hline Boundary conditions & \multicolumn{2}{l}{ Values and their range } \\
\hline \multirow{2}{*}{ Inlet velocity of the evaporator } & $\begin{array}{l}\text { varying stroke }(\mathrm{cm}) \\
\text { frequency }(\mathrm{n})\end{array}$ & $7.62,8.62,9.62,10.62$ \\
Inlet velocity of the condenser & $1.223 \mathrm{~m} / \mathrm{s}$ & \\
Inlet temperature of the & $283 \mathrm{~K}$ & \\
condenser & $0.0001 \mathrm{~m}$ & \\
Solid wall thickness & Heat $(\mathrm{W})$ & Average Heat Flux $\left(\mathrm{W} / \mathrm{m}^{2}\right)$ \\
& 551 & 7983 \\
& 500 & 7244 \\
Heat $(\mathrm{Q})$ & 455 & 6592 \\
& 400 & 5795 \\
\hline
\end{tabular}


Table 4.5: Details of the Numerical solution variables.

\begin{tabular}{lc}
\hline Solver parameters & Solver parameters Values \\
\hline Solution Method & PISO \\
Time step (s) & 0.5 \\
Relaxation factor momentum & 0.4 \\
Relaxation factor pressure & 0.3 \\
Relaxation factor energy & 0.9 \\
Turbulence kinetic energy & 0.5 \\
Turbulence viscosity & 0.5 \\
Turbulence dissipation rate & 1 \\
Density & 1 \\
\hline
\end{tabular}


For the turbulent flow, the k- $\varepsilon$ turbulent model is used. The boundary and operating conditions are described in Table 4.3 and Table 4.4. Details of the numerical solution scheme are presented in Table 4.5. The CFD numerical algorithm utilizes Pressure Implicit with Splitting of Operators (PISO) formulation for the solution of the Navier-Stokes and heat transport equations. Gradient discretization was Green-Gauss Node based, pressure discretization was Second order while the momentum and energy discretization were Second order upwind. The turbulent Kinetic Energy and turbulent dissipation were First order upwind using the k- $\varepsilon$ turbulence model. The transient formulation was second order Implicit. Appropriate under relaxations were used to improve the numerical stability for all governing equations. The criteria for convergence was set at $10^{-4}$ for the continuity, $x, y$ and $z$ momentum, turbulent kinetic energy and turbulence dissipation rate. A convergence criterion of $10^{-8}$ was set for the DPDHL and $10^{-6}$ for the RMDHL.

The geometry and thermal boundary conditions for the RMDHL is a bit more complicated than the DPDHL. DPDHL will have permanent inflow and outflow regions, where one can define the inlet velocity and temperature boundary conditions. RMDHL flows require interchange between the inflow and out-flow boundaries during a cycle. For most applications, it is difficult to determine the inflow/outflow boundary conditions, since fluid particles exiting the flow domain during a part of the cycle are fed back into the domain later in the cycle. The DPDHL which represents the case of forced convection heat transfer in channels and tubes is well understood. Several results in analytical and empirical relations for the Nusselt number variations regarding the 
flow parameters are available (Faghri, Zhang, and Howell 2010). The Geometry and grid distribution for RMDHL is the same as DPDHL.

As mentioned before, the heat fluxes applied on both sides of the evaporator as well as other operational variables and the boundary conditions that are numerically simulated are presented in Tables 4.3 and 4.4. Fig. 4.34(a) and (b) show the comparison of the numerical model and the experimental results for two different heat input conditions. It was observed that the results of the numerical model show a similar trend to the results of the experimental measurements. The mesh independence studies were conducted for two more Grids with coarse and fine meshes settings in ANSYS Fluent (Table 4.6), resulting in a variation of less than $5 \%$ for the temperature variation on the plate surface. The results of the computational study were, generally, in good agreement with experimental data, signifying a level of confidence with the present study. Hence the numerical solution was used as the basis for the result analysis. Fig.4.35 and Fig. 4.36 show the contour of varying temperature across evaporator for varying heat transfer rate and at different stages in the numerical simulation. The figures show the temperature gradients expected in such numerical analysis, which is heat enters the system from the cold plate and the heat is removed from the system at the condenser. 


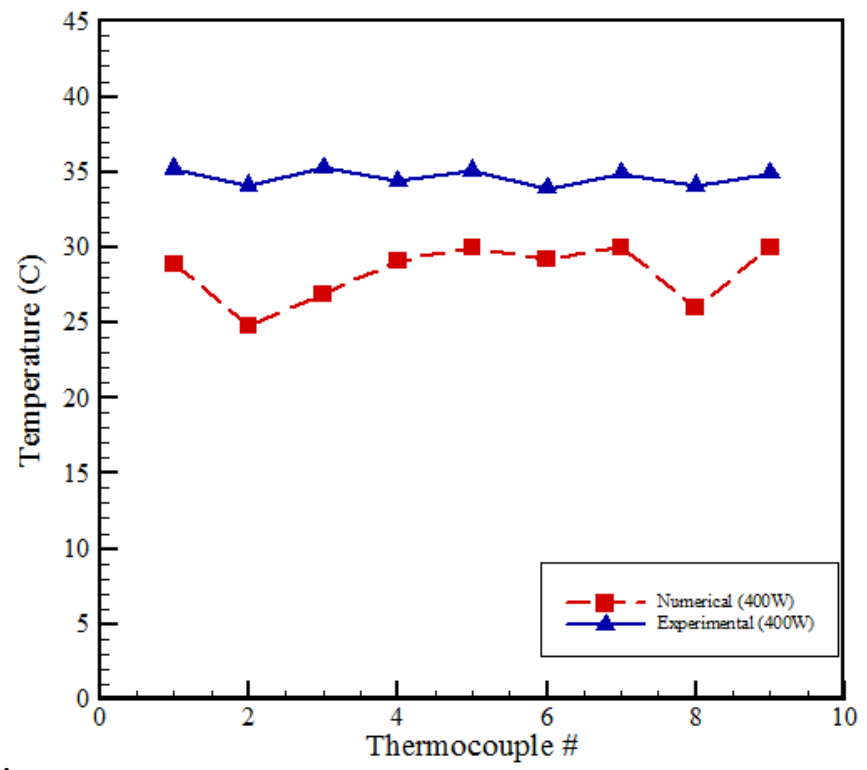

(a)

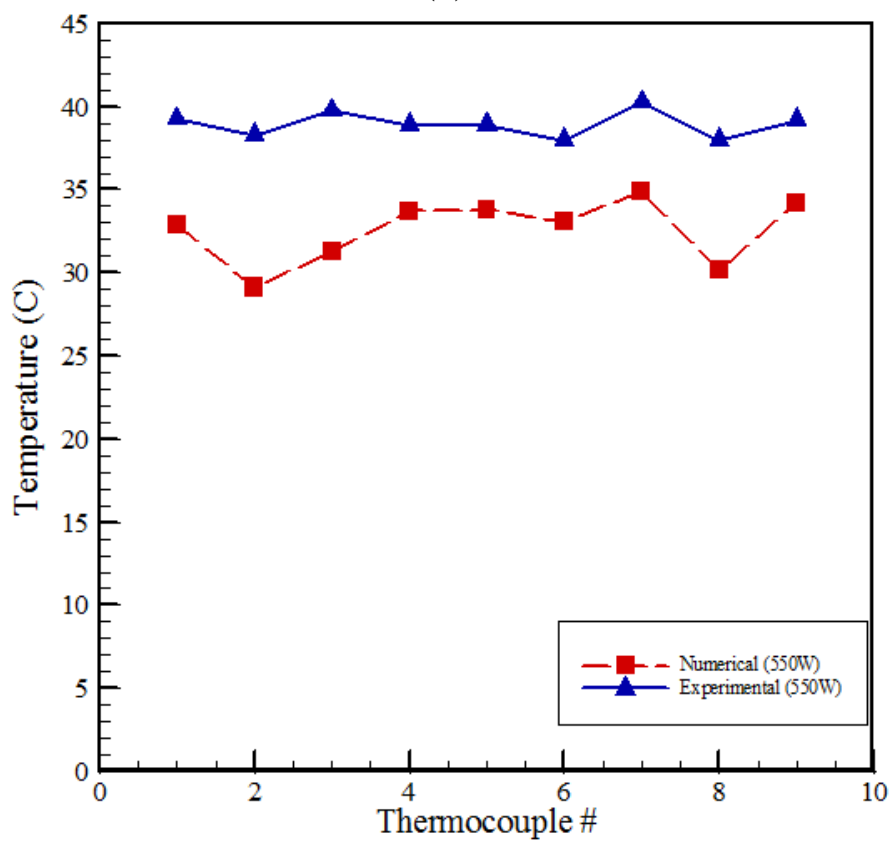

(b)

Figure 4. 34: Comparison of numerical and experimental Temperature distributions over the cold plate at different heat inputs for a coolant inlet temperature of $10^{\circ} \mathrm{C}$. 
Table 4. 6: Mesh Information for Numerical investigation.

\begin{tabular}{|l|l|l|l|}
\hline Domain & Nodes & Tetrahedron elements & Total elements \\
\hline body part & 46978 & 167499 & 167499 \\
\hline condenser part & 88243 & 165196 & 275326 \\
\hline evaporate part & 129837 & 149526 & 338421 \\
\hline All Domains & 265059 & 482221 & 781246 \\
\hline
\end{tabular}

Q

RMDHL

DPDHL

(W)

550

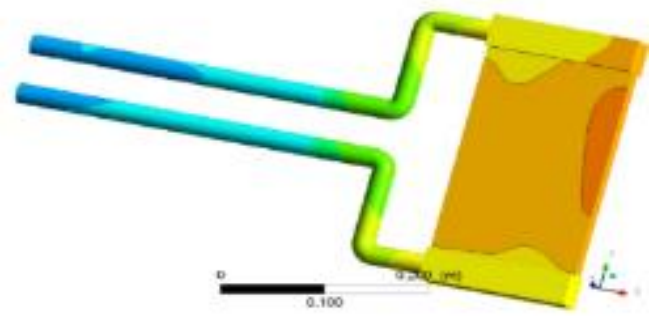

(a)

450

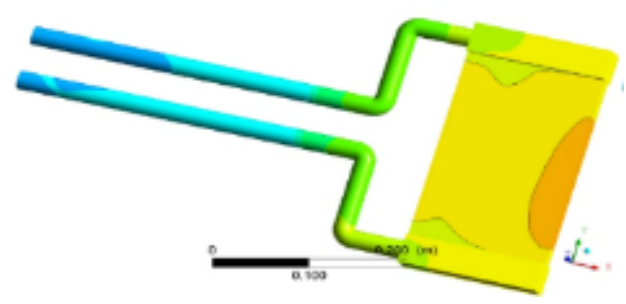

(c)

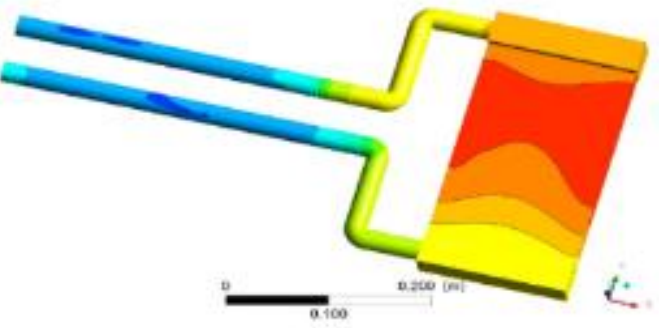

(b)

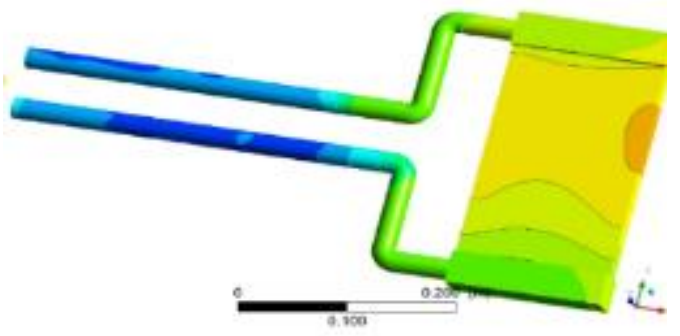

(d)

Figure 4. 35: The contour of varying temperature across evaporator for varying heat transfer rates. 


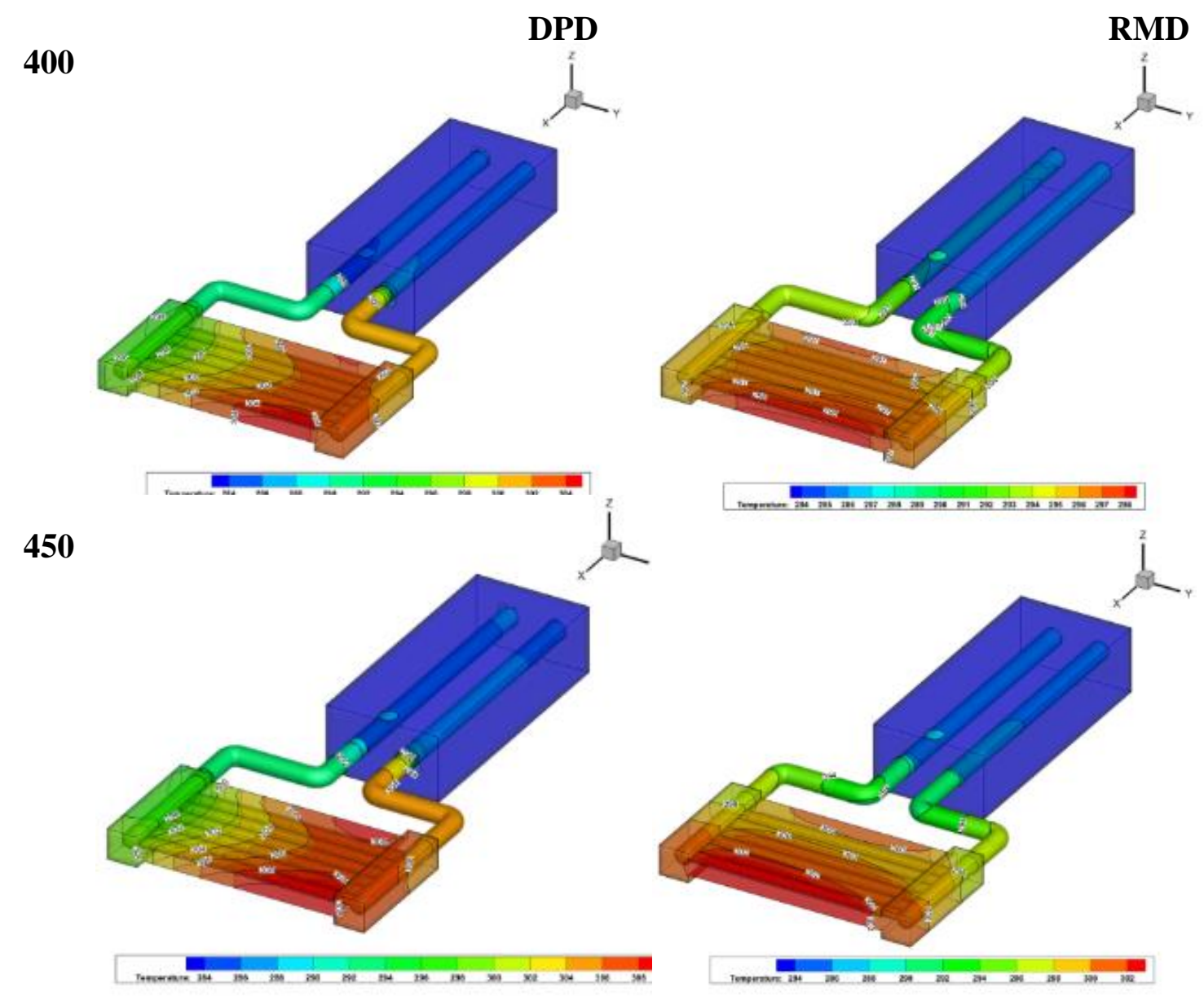



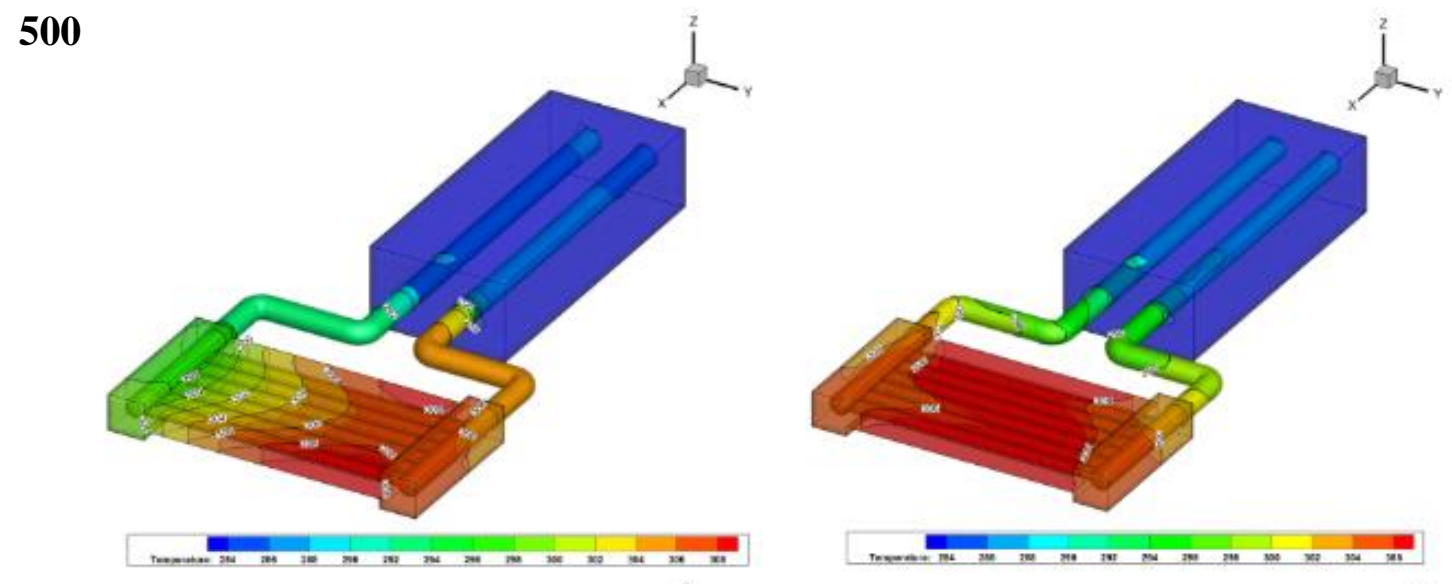

550

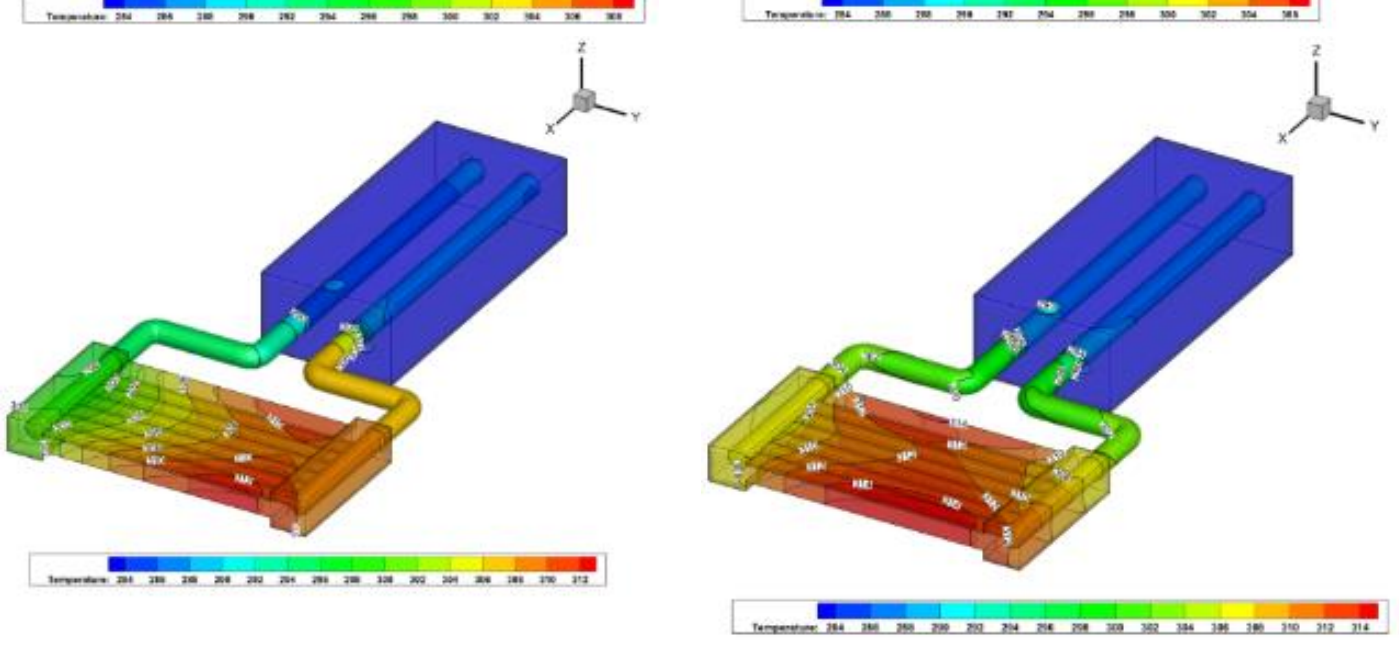

Figure 4. 36: Temperature contours of hot plate for various heat transfer rate and cooling methods 


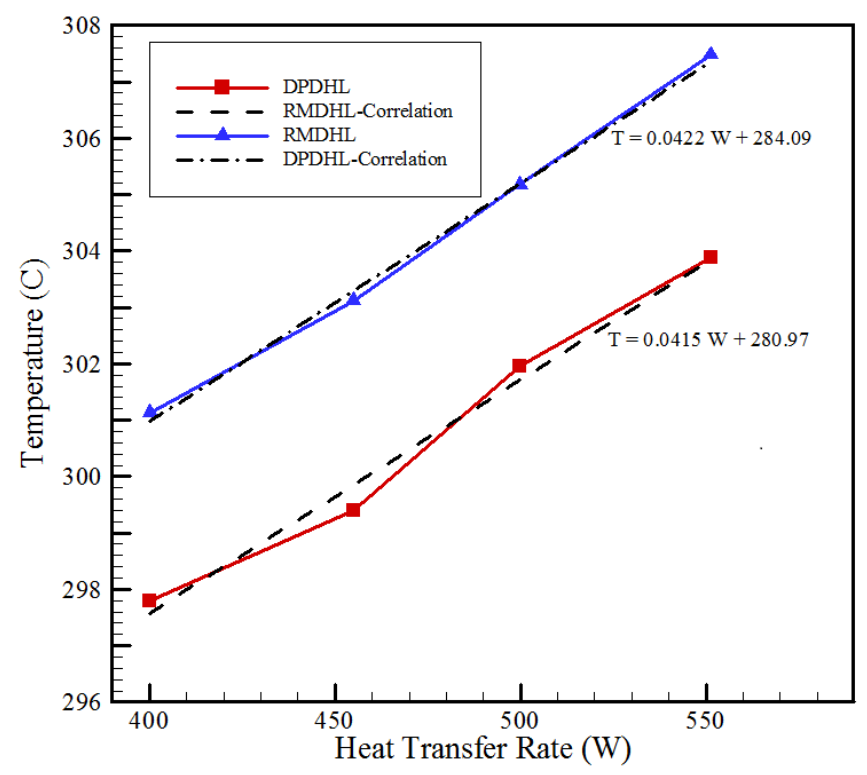

Figure 4. 37. The plot of Heat transfer rate vs. average temperature.

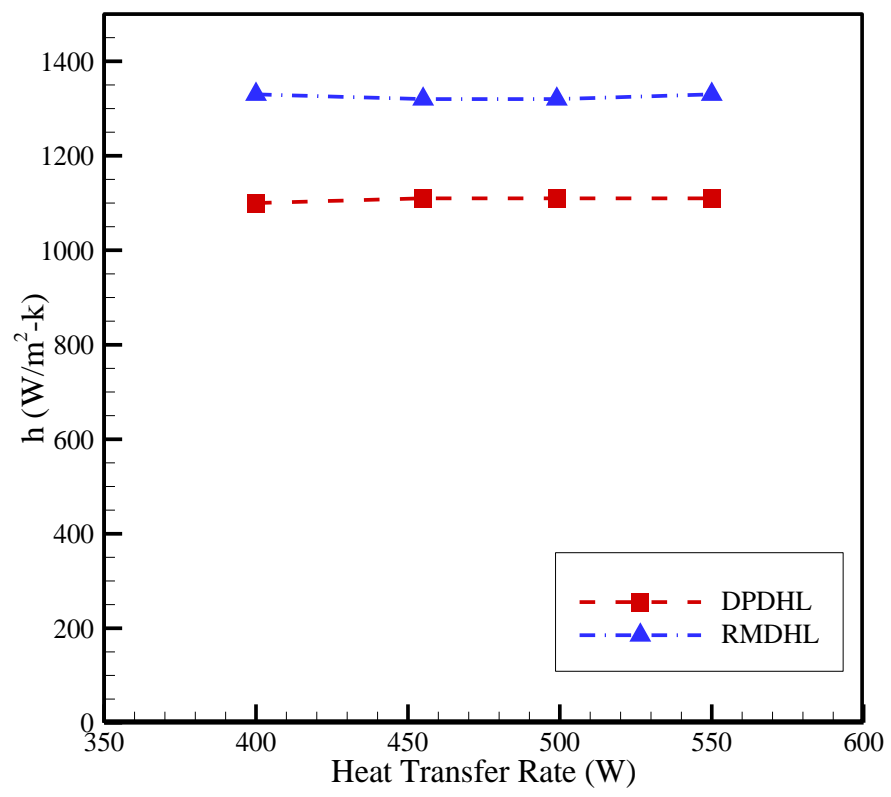

Figure 4. 38: The Plot of Heat transfer coefficients for both RMDHL and DPDHL at different heat transfer rate. 
For the RMDHL, the temperature profile is more uniform, and the maximum temperature occurs at the midpoint of the walls. As expected, average hot plate temperature for RMDHL is lower than the average cold plate temperature for the DPDHL for all heat flux and volume flow rates observed. The difference in the temperature is in some cases up to $3.5^{\circ} \mathrm{C}$ while surface temperature differences at specific certain locations were up to $8^{\circ} \mathrm{C}$, as shown in Fig.4.35 and Fig 4.36, for heat transfer rates of 551, 500, 455, 400 watts. The DPDHL produced an average hot plate temperature of $307.409^{\circ} \mathrm{C}, 305.102^{\circ} \mathrm{C}, 303.123^{\circ} \mathrm{C}$, and $301.115^{\circ} \mathrm{C}$ respectively, while the RMDHL produced an average of $303.871{ }^{\circ} \mathrm{C}, 301.944{ }^{\circ} \mathrm{C}, 299.447{ }^{\circ} \mathrm{C}$, and $297.847^{\circ} \mathrm{C}$ respectively.

The observed difference in the mean cold plate temperature has been predicted by (Grossman and Nachman 2006). Because of the same mean fluid velocity for the DPDHL and the RMDHL, the expectation that the more rigorous breakdown of the thermal boundary layer by the reciprocating fluid motion will similarly enhance heat exchange in the turbulent reciprocating flow of the RMDHL, producing more effective heat transfer than in a continuous flow in the DPDHL.

Fig. 4.39 show the temperature distributions of both the RMDHL and DPDHL cold plates at a condenser inlet velocity of $1.23 \mathrm{~m} / \mathrm{s}$, heat transfer rate ranges from $400 \mathrm{~W}$ to $550 \mathrm{~W}$ and a condenser inlet temperature of $283 \mathrm{~K}$. with other parameters as discussed in Table 4.5. The uniformity of the temperature profiles of the RMDHL, shown in the left column, is much better than that of the DPDHL. As seen in Fig 4.39 (a)-(h), there are two temperature gradients, one gradient along the evaporator width 


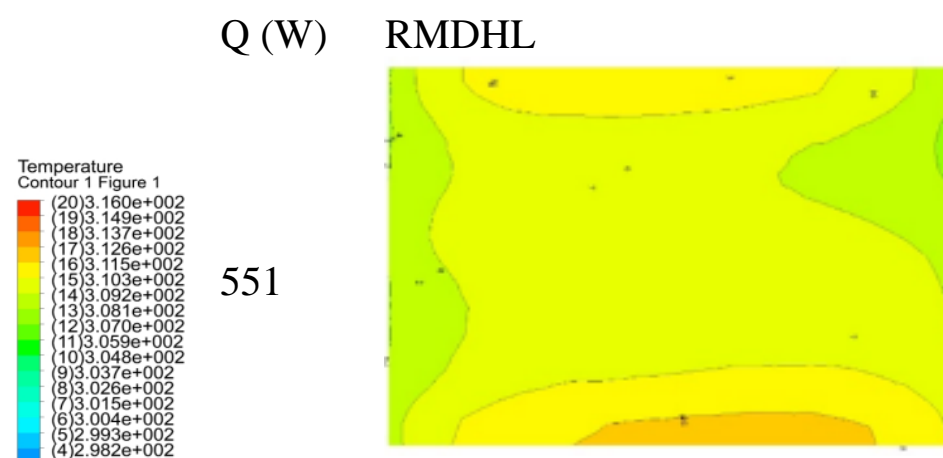

DPDHL

(a)

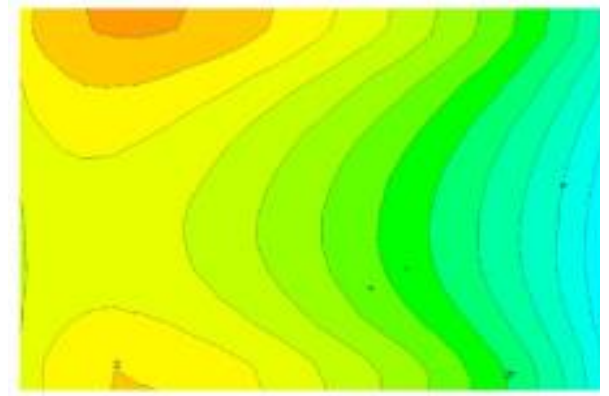

(1) $2.950 \mathrm{e}+002$

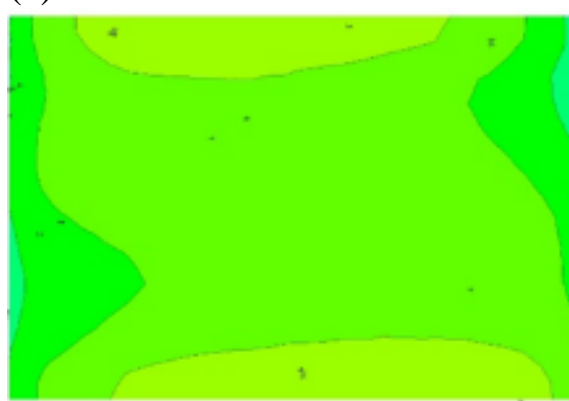

(b)

500

(C)

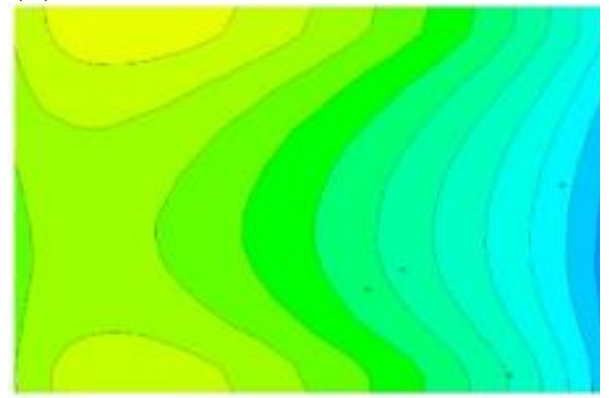

(d)

455
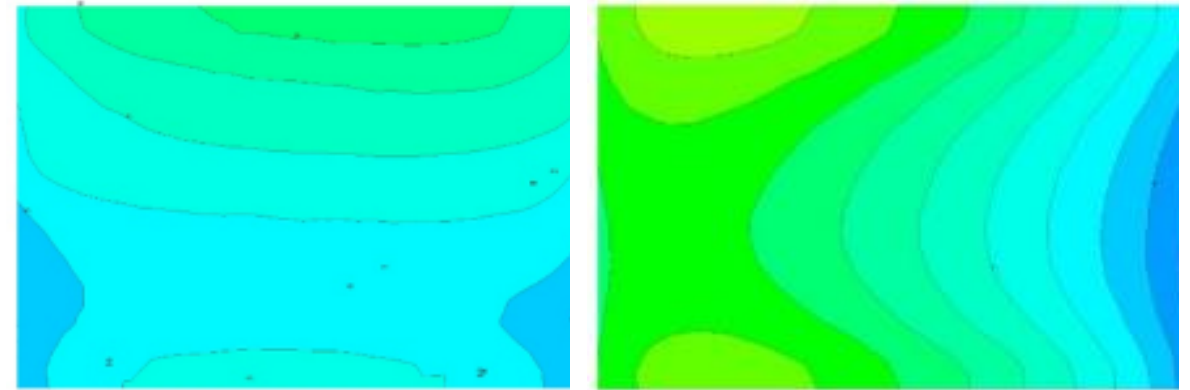

(e)

(f)

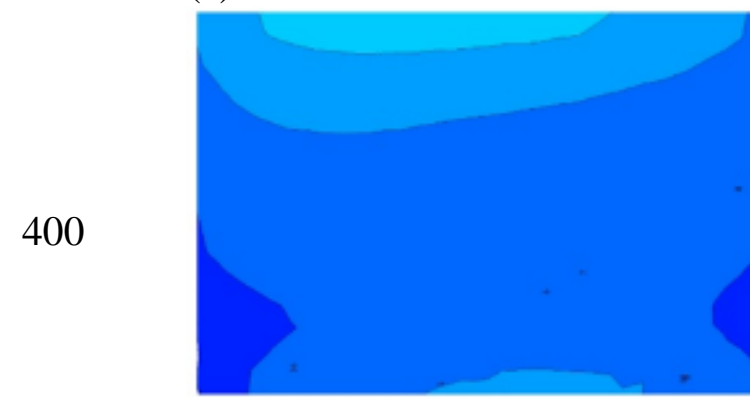

(g)

(h)

Figure 4. 39: Temperature contours of hot plate for various heat transfer rate and cooling methods 
and the other across the evaporator thickness. The gradient along the evaporator width is similar for both the DPDHL and the RMDHL due to the pressure drop along the grooves in the evaporator.

The effect of this gradient can be reduced if a different groove configuration is adopted. However, it is observed that the pressure gradient along the evaporator is more pronounced for the DPDHL than the RMDHL. For the temperature gradient along the fluid flow path, Fig. 4.39 shows that the DPDHL (the column on the right) has up to 13 distinct temperature bands, as observed for the 551W cold plate. It was also observed that temperature band increases with increasing heat flux. Whereas for the RMDHL the number of bands is much fewer and independent of heat flux. As much as $80 \%$ of the cold plate of the RMDHL was maintained at a temperature within a difference of $0.5^{\circ} \mathrm{C}$.

To show the statistical variation of this temperature distribution, three cross sections are examined as shown in Fig.4.40. Section A-A at one end, Section B-B at the center, and section $\mathrm{C}-\mathrm{C}$ at the other end. Temperatures at the centerline were compared for both the RMDHL and DPDHL at two heat transfer rates of 400 and 550 W. Fig. 4.41, Fig. 4.42 and Table 4.7 present the details of the analysis. The plate temperature is minimum at the inlet of the evaporator and gradually increases toward the outlet of the DPDHL flow loop. However, the temperature distribution is substantially more uniform along the width for the RMDHL. 


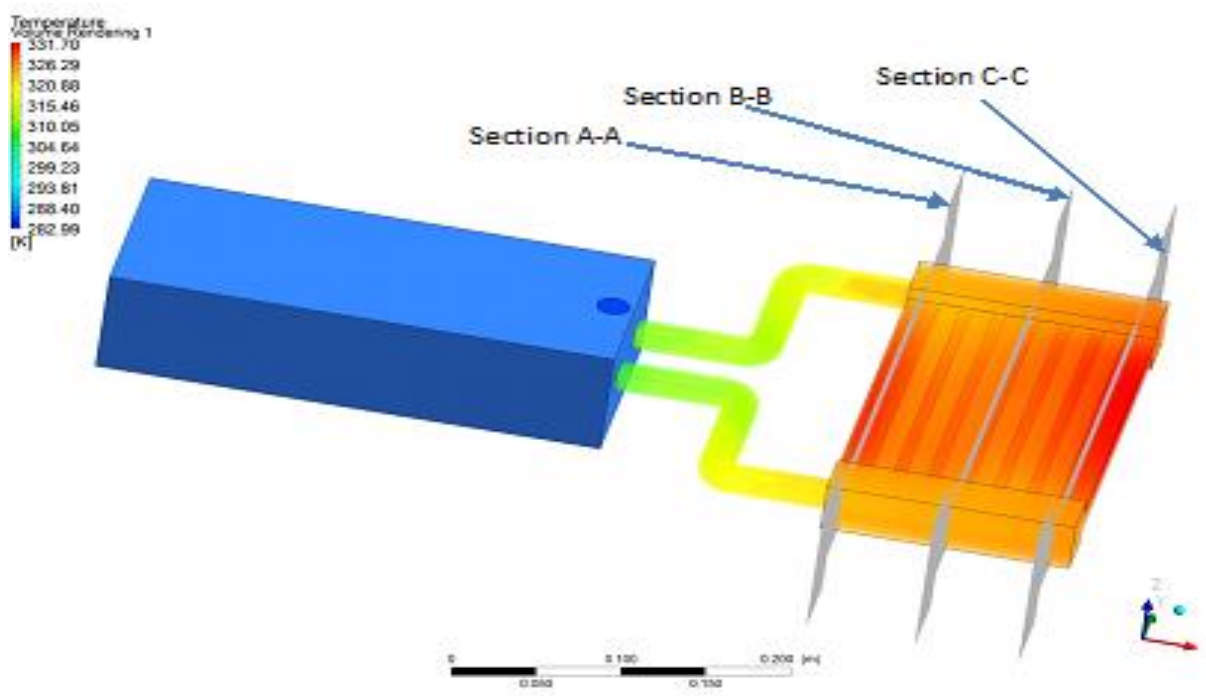

Figure 4. 40. Volume rendering of a cross-section of the hot plate showing cross section for temperature investigation. 


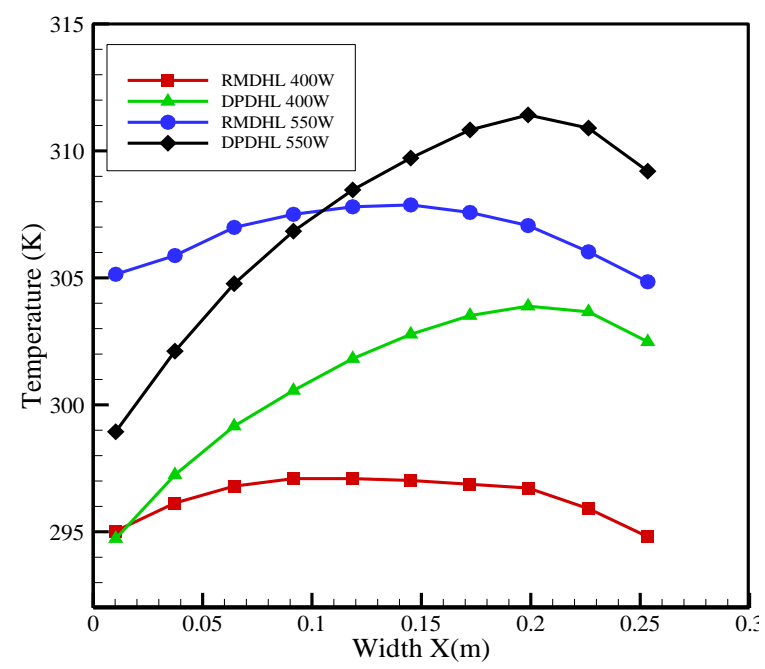

(a)

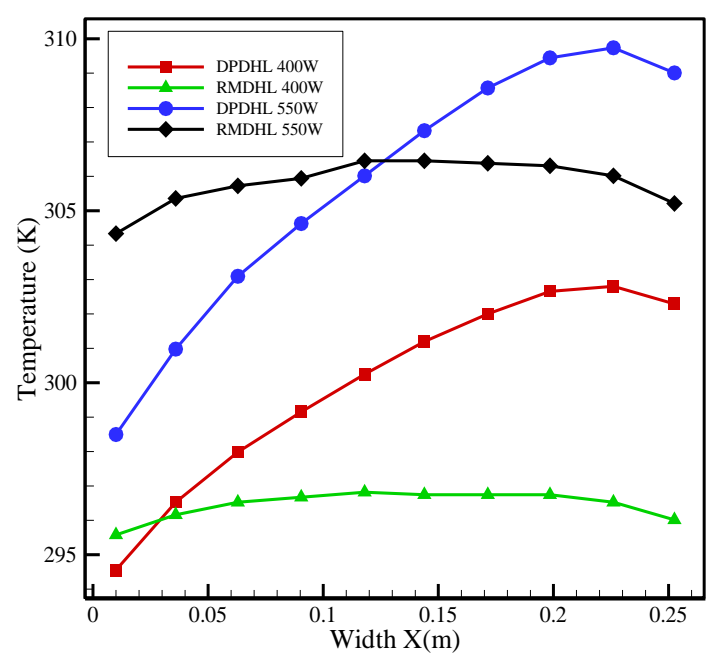

(b)

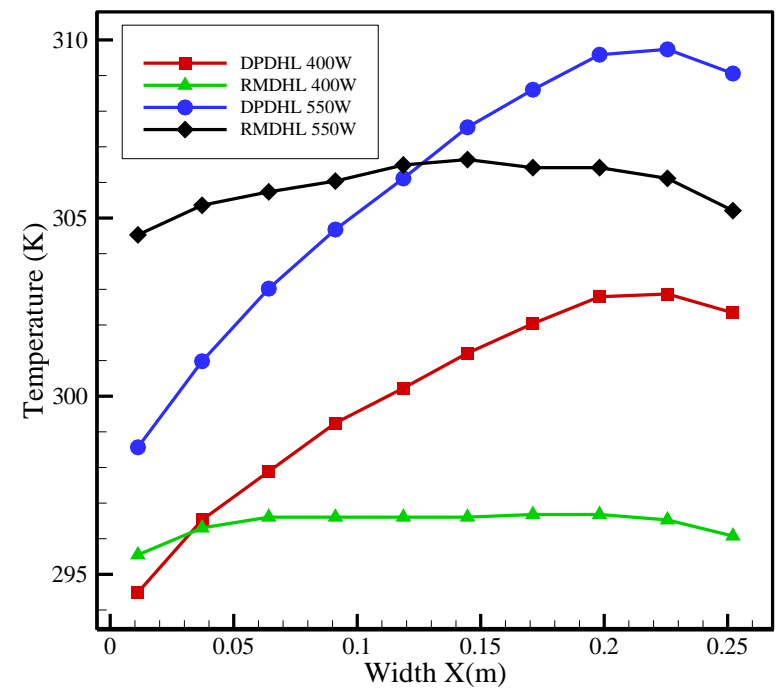

(c)

Figure 4. 41. Temperature profile for a cross-section along hot plate (a) section A-A (b) section B-B (c) section C-C 
Table 4. 7: Bulk Temperature variation across various cross-sectional areas of the hot plate.

\begin{tabular}{|l|c|c|c|c|c|c|c|c|c|c|c|c|}
\hline & \multicolumn{9}{|c|}{ DPDHL } & \multicolumn{5}{c|}{ RMDHL } \\
\hline $\mathrm{Q}(\mathrm{W})$ & \multicolumn{3}{|c|}{400} & \multicolumn{3}{|c|}{550} & \multicolumn{3}{c|}{400} & \multicolumn{3}{c|}{550} \\
\hline $\mathrm{x}(\mathrm{m})$ & $\mathrm{T} \min$ & $\mathrm{T} \max$ & $\Delta \mathrm{T}$ & $\mathrm{T} \min$ & $\mathrm{T} \max$ & $\Delta \mathrm{T}$ & $\mathrm{T} \min$ & $\mathrm{Tmax}$ & $\Delta \mathrm{T}$ & $\mathrm{Tmin}$ & $\mathrm{Tmax}$ & $\Delta \mathrm{T}$ \\
\hline 0.01 & 291.7 & 304.1 & 12.4 & 294.5 & 311.5 & 17.0 & 293.5 & 297.3 & 3.8 & 303.4 & 311.2 & 7.8 \\
\hline 0.03 & 291.7 & 303.7 & 12.0 & 294.5 & 310.9 & 16.4 & 293.8 & 296.7 & 2.9 & 303.7 & 310.4 & 6.7 \\
\hline 0.04 & 291.7 & 303.0 & 11.3 & 294.5 & 310.0 & 15.5 & 294.3 & 296.6 & 2.3 & 304.4 & 309.5 & 5.1 \\
\hline 0.06 & 291.8 & 303.0 & 11.2 & 294.6 & 310.0 & 15.4 & 294.6 & 296.8 & 2.2 & 307.0 & 309.7 & 2.7 \\
\hline 0.08 & 291.9 & 303.2 & 11.3 & 294.7 & 310.3 & 15.6 & 294.7 & 296.9 & 2.2 & 307.0 & 309.8 & 2.8 \\
\hline 0.10 & 292.1 & 303.6 & 11.5 & 295.1 & 310.9 & 15.8 & 295.0 & 297.2 & 2.1 & 307.1 & 310.0 & 2.9 \\
\hline 0.12 & 292.7 & 304.5 & 11.8 & 295.8 & 312.1 & 16.3 & 295.2 & 298.0 & 2.8 & 307.4 & 310.9 & 3.5 \\
\hline 0.14 & 295.8 & 305.0 & 9.20 & 300.1 & 312.7 & 12.6 & 295.60 & 298.5 & 2.9 & 307.9 & 311.7 & 3.8 \\
\hline
\end{tabular}

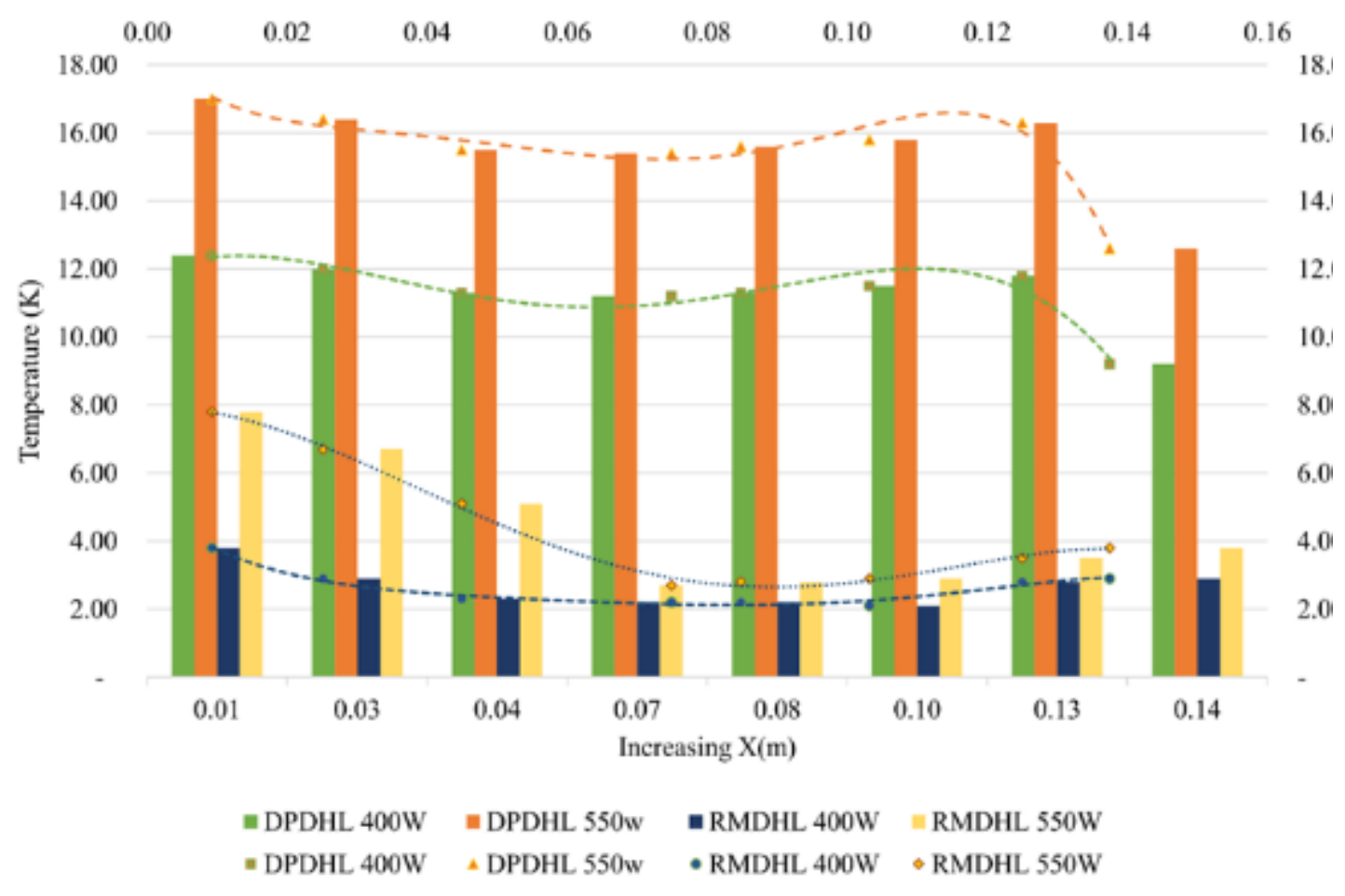

Figure 4. 42: Temperature variation across various cross-sectional areas of the hot plate. 
Table 4. 8: Standard deviation of temperature across cross-sections of the hot plate.

\begin{tabular}{lcccc}
\hline & & & $400 \mathrm{~W}$ & $550 \mathrm{~W}$ \\
& $400 \mathrm{~W}$ & $550 \mathrm{~W}$ & RMDHL & RMDHL \\
\hline Section A-A & 3.03 & 4.14 & 0.84 & 1.09 \\
Section B-B & 2.83 & 3.88 & 0.38 & 0.70 \\
Section B-B & 2.83 & 3.88 & 0.38 & 0.70 \\
\hline
\end{tabular}

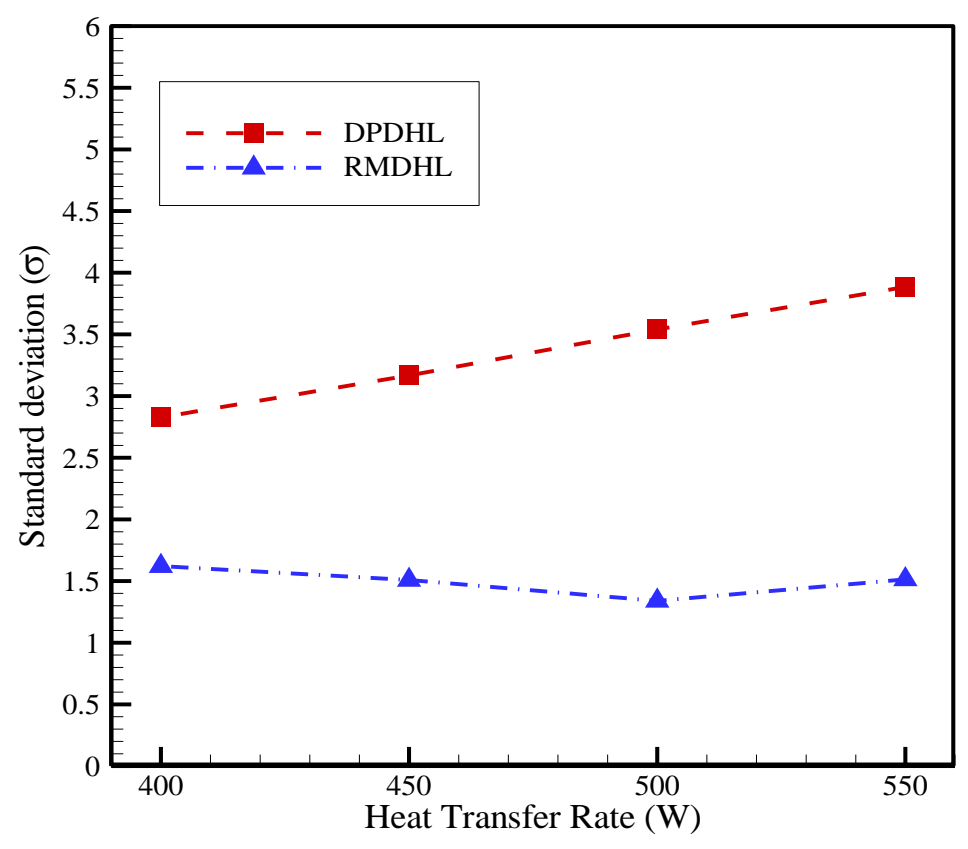

Figure 4. 43: Plot of Heat transfer rate and standard deviation 
Another statistic indicator that may be used to gauge the temperature uniformity over the cold plate is the standard deviation, defined by (Cao, $\mathrm{Xu}$, and Gao 2013):

$$
\sigma_{T}=\sqrt{\frac{1}{N-1} \sum_{i=1}^{N}\left(T_{i}-T_{m}\right)^{2}}
$$

For this purpose, the steady-state minimum temperature, maximum temperature, and the temperature difference at different power inputs for water coolant inlet temperatures of $10^{\circ} \mathrm{C}$ are shown in Fig.4. 42.

Although the maximum temperature differences may vary with the power input, they are generally around $6^{\circ} \mathrm{C}$ for the RMDHL and $17^{\circ} \mathrm{C}$ for the DPDHL loop. The standard deviation for the RMDHL is less than half of the value for the DPDHL (Table 4.7). The standards deviation of the RMDHL changes only slightly with increasing rate of heat transfer, but for the DPDHL it increases significantly with increasing rate of heat transfer. It should be pointed out that all of the data presented herein are based on a low driver reciprocating frequency of $0.14 / \mathrm{s}$ for a maximum stroke. Fig. 4.42 show that not only is the mean temperature for the RMDHL lower than the mean temperature for the DPDHL, but the standard deviation around the mean is also about $1.5^{\circ} \mathrm{C}$ for the RMDHL and about $4^{\circ} \mathrm{C}$ for the DPDHL. Fig. 4.42 also reveals that although the variation in cold plate temperature is different for the RHMDL and the DPDHL, the temperature trend is the same. The temperature is the highest at the entrance, and it begins to drop as the cooling proceeds towards the outlet. The distribution is flattening towards the middle with a slight inflection and continues to fall at the outlet of the fluid. 


\subsection{Results of Two-Phase Heat Transfer Modeling}

The objective of the work in thi section is to numerically study a solenoidoperated RMDHL in connection with high heat flux thermal management applications. The second objective is to validate the numerical investigation with the experimental data. Due to the lack of suitable correlations for oscillating flow conditions in the literature, correlations derived from steady state unidirectional flow assumptions will be adopted and modified for the RMDHL flow ( Walther, Kühl, and Schulz 2000; Lee, Park, and Tack Ro 1998; T. S. Zhao and Cheng 1998b; T. S. Zhao and P.Cheng 1996) and the results obtained will be compared to experimental data.

Figure 4.44 provides a simplified schematic of geometry and boundary conditions for this study, which will be the basis for developing the models for the twophase RMDHL. The commercial ANSYS Fluent CFD code was employed to simulate the RMDHL numerically. The first step in the model development is the generation of a 3D CAD model by SolidWorks. Both the condenser and evaporator of the loops are enclosed with solid wall. The solid walls are copper, and their thermophysical properties are obtained from the fluent database. The working liquid in the RMDHL

loops is water. The model includes the loop condenser with specified inlet and outlet conditions associated with the condenser.

The operating and boundary conditions of the two-phase model are provided in Table 4.9. Details of the numerical solution scheme are presented in Table 4.10. The CFD numerical algorithm utilizes Pressure Implicit with Splitting of Operators (PISO) formulation for the solution of the Navier-Stokes and heat transport equations. Gradient 


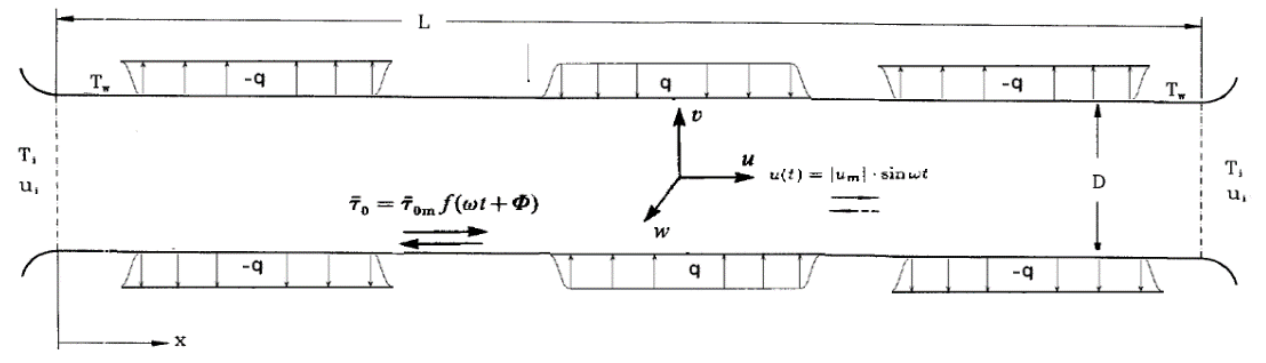

Figure 4. 44: The geometry and boundary conditions for this study

Table 4. 9: The boundary conditions for the governing equations

\begin{tabular}{|c|c|c|}
\hline Boundary conditions & \multicolumn{2}{|c|}{ Values and their range } \\
\hline \multirow{2}{*}{ Inlet of the evaporator } & stroke $(\mathrm{cm})$ & 7.62 \\
\hline & frequency $(\mathrm{Hz})$ & 1 \\
\hline \multirow{8}{*}{$\begin{array}{l}\text { Inlet velocity of the } \\
\text { condenser }(\mathrm{m} / \mathrm{s}) \\
\text { Solid wall thickness } \\
\text { (m) }\end{array}$} & 0.69 & \\
\hline & 0.0005 & \\
\hline & $\begin{array}{l}\text { Inlet temp of the } \\
\text { condenser }(K)\end{array}$ & Heat $(W)$ \\
\hline & & 171 \\
\hline & 212 & 156 \\
\hline & 315 & 126.9 \\
\hline & & 75.9 \\
\hline & & 162 \\
\hline \multirow{7}{*}{ Heat (Q) } & & 131.4 \\
\hline & 323 & 104 \\
\hline & & 79.1 \\
\hline & & 166 \\
\hline & 230 & 137.2 \\
\hline & 338 & 107.4 \\
\hline & & 81.6 \\
\hline
\end{tabular}


Table 4. 10: Details of the Numerical solution variables

\begin{tabular}{ll}
\hline Solver parameters & Solver parameters \\
\hline Solution Method & Coupled \\
pressure-Velocity Coupling & Full Multiphase \\
& Coupled \\
Spatial discretization schemes & Second order Upwind \\
Transient formulation & Second order Implicit \\
Time step (s) & 0.05 \\
Relaxation factor momentum & 0.4 \\
Relaxation factor pressure & 0.3 \\
Relaxation factor energy & 0.4 \\
Relaxation factor Turbulence kinetic & 0.4 \\
energy & \\
Relaxation factor Turbulence viscosity & 0.4 \\
Turbulence dissipation rate & 0.6 \\
Relaxation factor Density & 1 \\
Relaxation factor body forces & 0.5 \\
Relaxation factor Vaporization mass & 1 \\
Flow Courant Number & 20 \\
Explicit Relaxation & 0.1 \\
\hline
\end{tabular}


discretization was Green-Gauss Node based, and pressure discretization was second order while the momentum and energy discretization were second-order upwind. The turbulent kinetic energy and turbulent dissipation were first order upwind using the k$\varepsilon$ turbulence model. The transient formulation was second order implicit. Appropriate under-relaxations were used to improve the numerical stability for all governing equations. The criteria for convergence was set at $10^{-3}$ for the continuity equation, $x, y$ and $z$ momentum equations, and turbulent kinetic energy and turbulence dissipation rate equations, while a convergence criterion of $10^{-6}$ was set for the energy equation.

Figure 45 (a) shows the open loop geometric configuration for the numerical model of the RMDHL, the location of the planes for data analysis, and grid distribution for numerical simulations, which was discretized with 500,000 polyhedral computational volumes. The resulting computational mesh is presented in Fig. 45(c). Temperature measurement was done over six planes. The planes are indicated and labeled as 1 to 6 in Fig. 45(b) according to the dimensions provided in Fig. 4.13. Mesh independence studies were conducted for two more grids with coarse and fine meshes settings in ANSYS Fluent to establish the computational accuracy of the computational domain. The resulting variation is less than $3.5 \%$ concerning temperature variation on the plate surface results, similar to other reported studies (O. T. Popoola and Cao 2015).

Figure 46 shows the temporal contours of vapor fraction in the numerical analysis at $212.05 \mathrm{~s}$ spaced with $0.1 \mathrm{~s}$ time step. In the forward direction Fig 4.46 (a) (c), the figure shows the increase in vapor fraction as a result of the absorption of heat at the evaporator. The continuous stream of vapor is transported to the condenser. The 


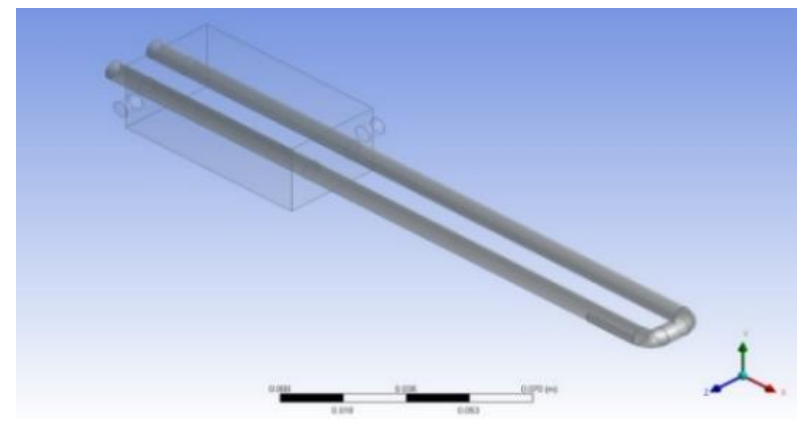

(a)

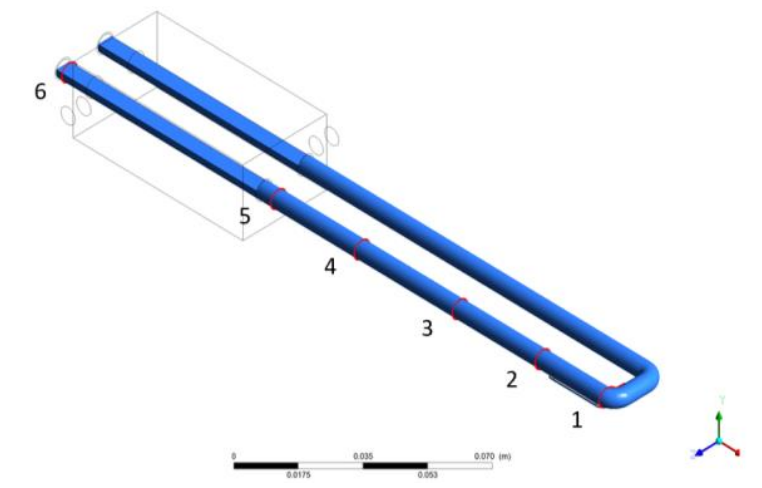

(b)

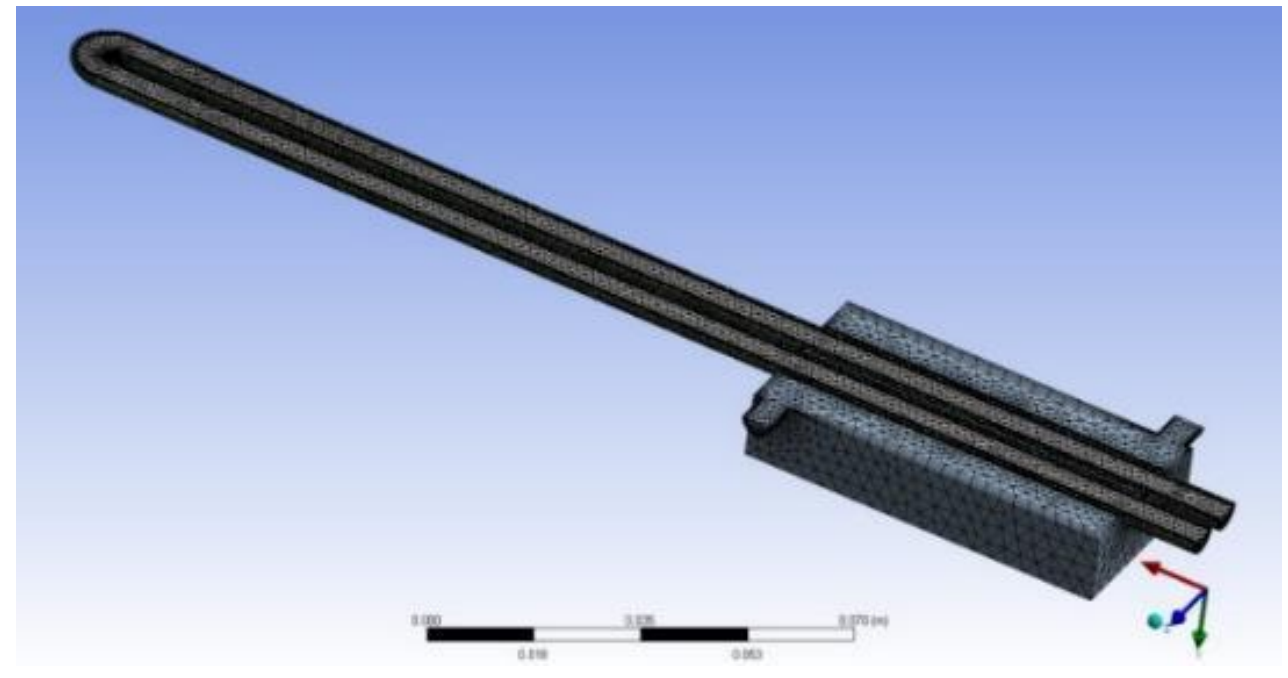

(c)

Figure 4. 45: (a) Open loop geometry configuration for numerical model of the RMDHL, (b) location of the planes for data analysis of the RMDHL, and (c) Grid distribution for numerical simulations of the RMDHL 


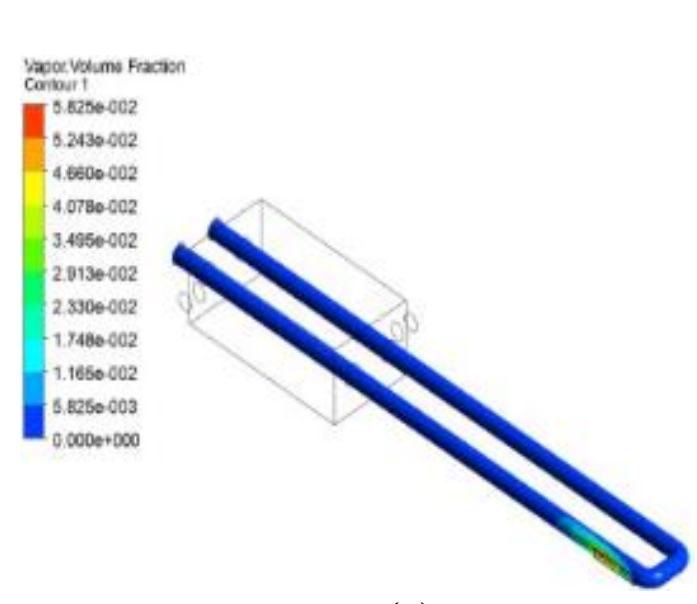

(a)

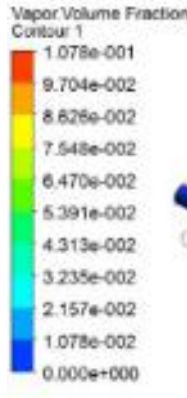

Wose.Volume Fiaction

2 Coste-00t

$1.32 \mathrm{Be}-001$

1E250.001

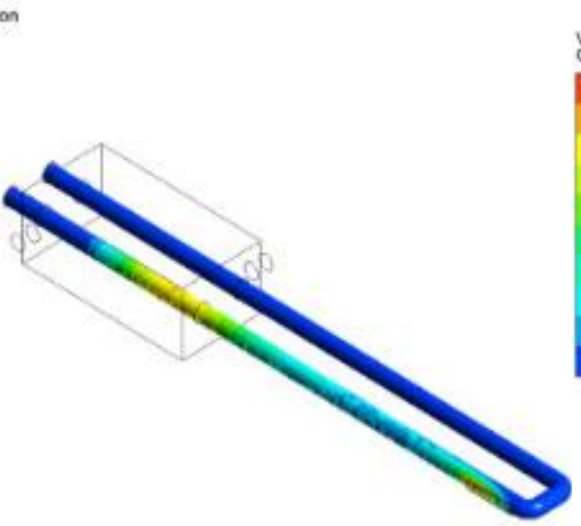

(c)

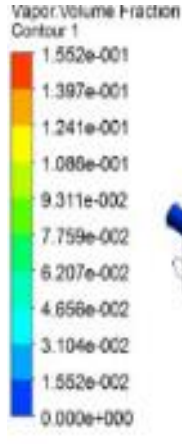

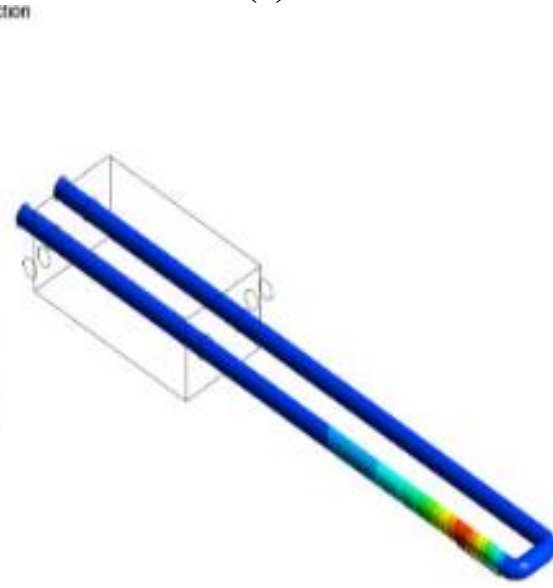

(e)
1.4220-001

12100001

reve 001

8. 1250.002

$6094 \mathrm{e}-002$

46630.002

$2031 \mathrm{e}-002$

$0,0050+000$

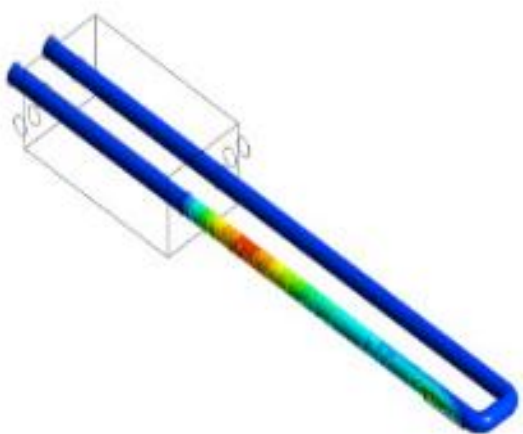

(b)

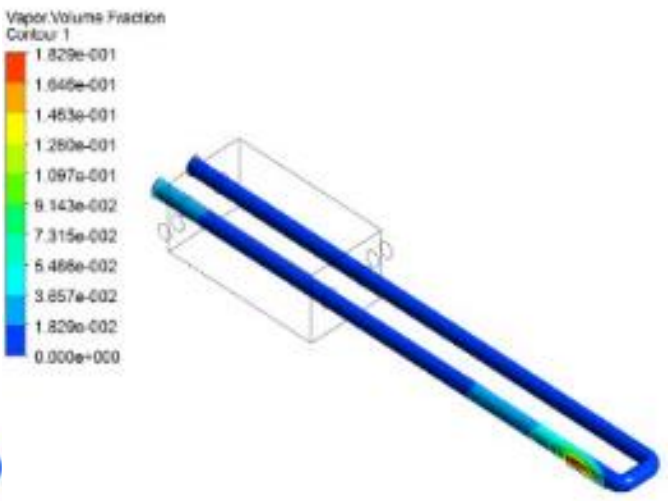

(d)

YaserVbume Fractsen
Conbur 1

$90750-801$

87110001

$7745 e 001$

- $67750-00$ !

5 ap7e 001

$48400-00$

-3372000 !

$29040-001$

1.896000

96790.000

$0.0006 \times 000$

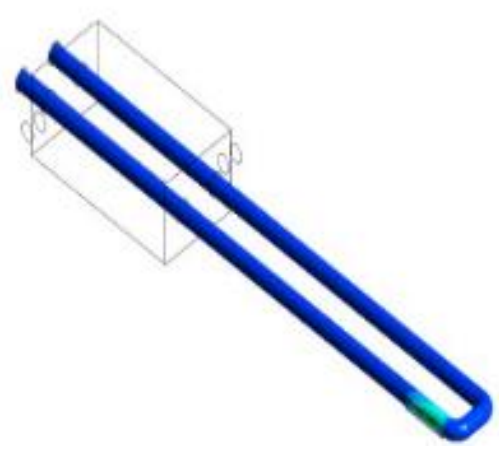

(f) 

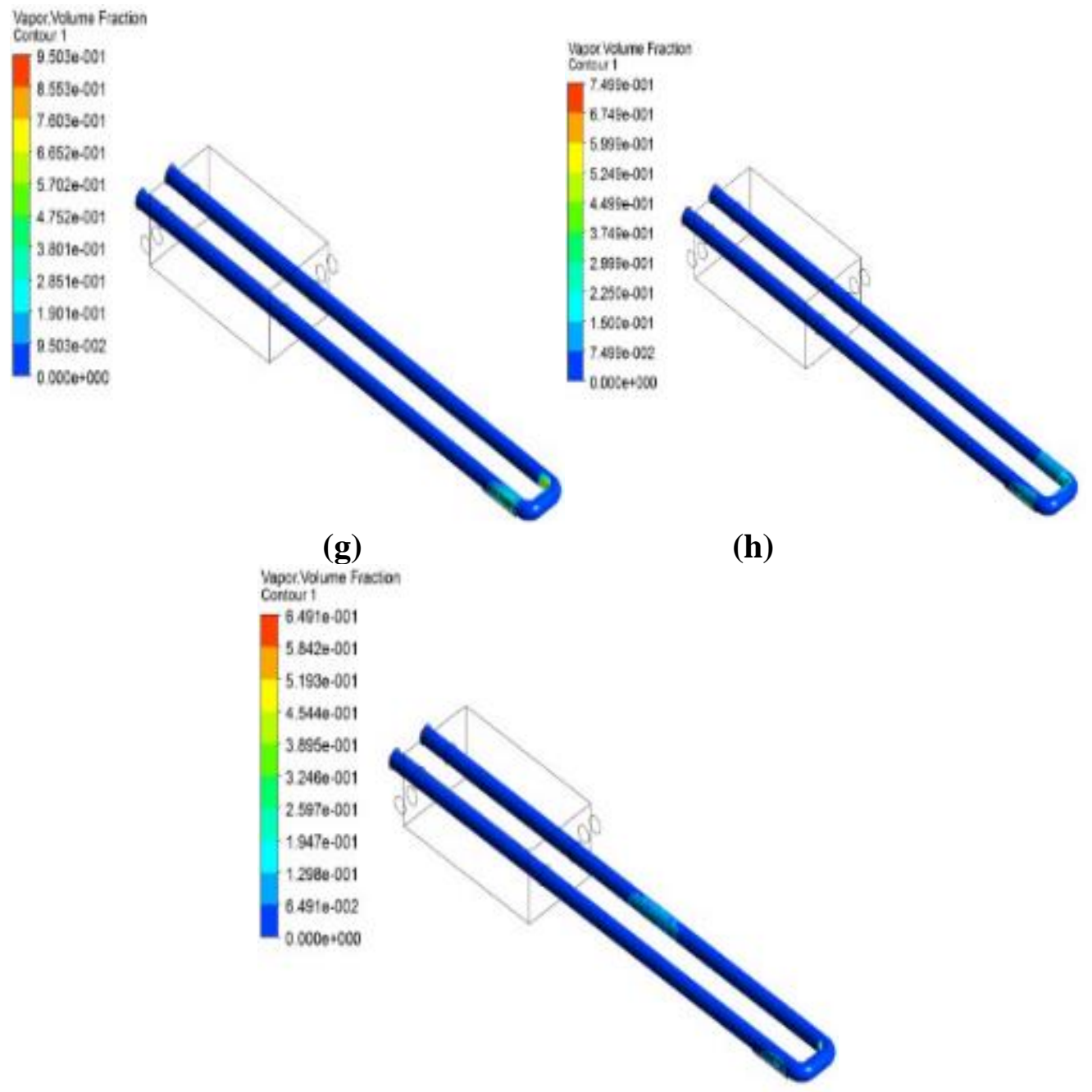

(h)

(i)

Figure 4. 46: Contour of vapor volume fraction in the evaporator at (a) 212.05 s (b) $212.15 \mathrm{~s}$ (c) $212.35 \mathrm{~s}$ (d) $212.50 \mathrm{~s}$ (e) $213.05 \mathrm{~s}$ (f) $212.35 \mathrm{~s}$ (g) $213.50 \mathrm{~s}$ (h)214.05 s (i) 214 
vapor fraction decreases along the condenser as the flow slows down and begins to change flow direction, accompanied by mixing and the vapor detaches in the loop. The model also captures the mixing that occurs due to an acceleration of flow around the bend in the reverse direction.

For this analysis, a time-based convergence criterion was selected to determine the conditions for convergence. It was observed that the results of the numerical simulation show a similar trend to the results of the experimental measurements. Figures 4.47 and 4.48 show the comparison of the numerical model and the experimental results for two condenser temperatures and varying levels of indicated heat input conditions using standard error bars. As indicated by the error bars, the agreement between the numerical results and experimental data are excellent, indicating the validity of the numerical model developed in this paper.

Figure 4.49 shows the contour of varying temperature along the heat loop for various heat transfer rates. The figure shows the typical temperature gradients between the evaporator, where heat enters the system from the heater, and the condenser where the heat is removed from the system. In a conventional heat loop where a unidirectional positive displacement mechanism is applied, it is normal to observe regions of stratified temperature gradients in the working fluid. These temperature gradients are associated with the thermal and velocity boundary layers. In the case of the RMDHL shown in Fig 4.49, such distinct temperature regions are absent in the fluid due to the mixing that occurs as a result of the reciprocating flow. 


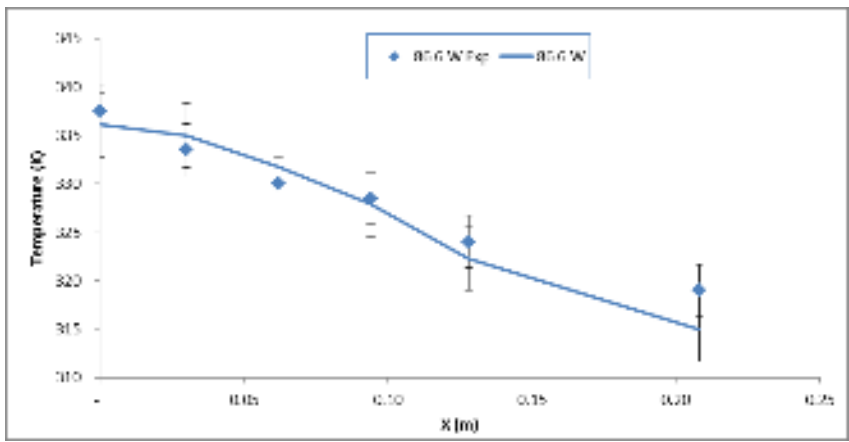

(a)

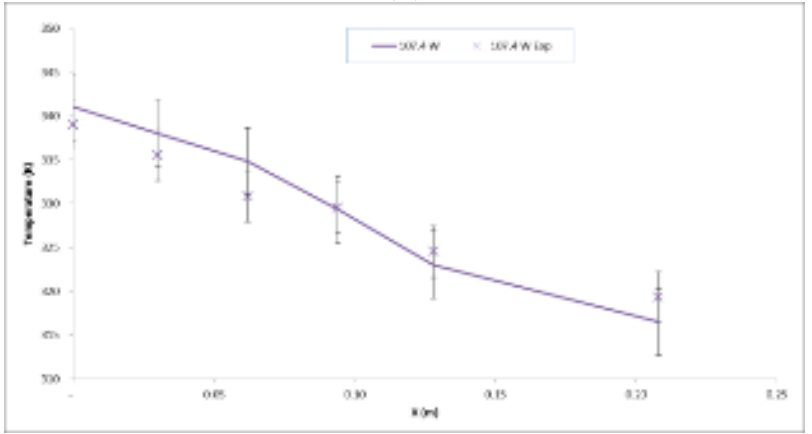

(b)

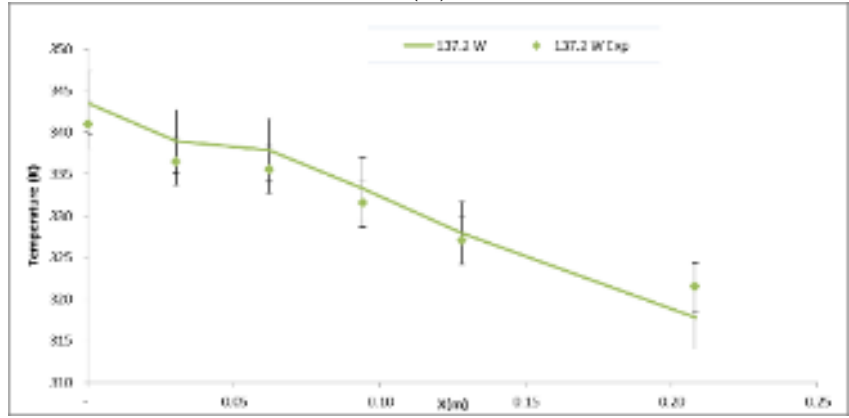

(c)

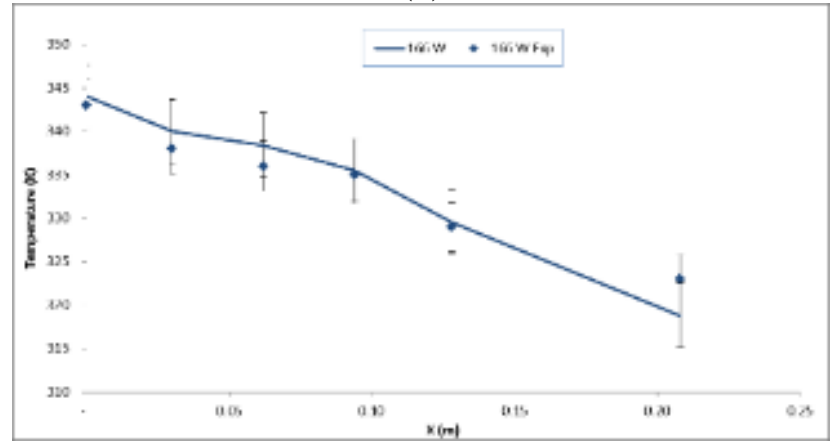

(d)

Figure 4. 47: Comparison of numerical and experimental temperature distributions along the heat loop at different heat inputs for a coolant inlet temperature of $313^{\circ} \mathrm{C}$. 


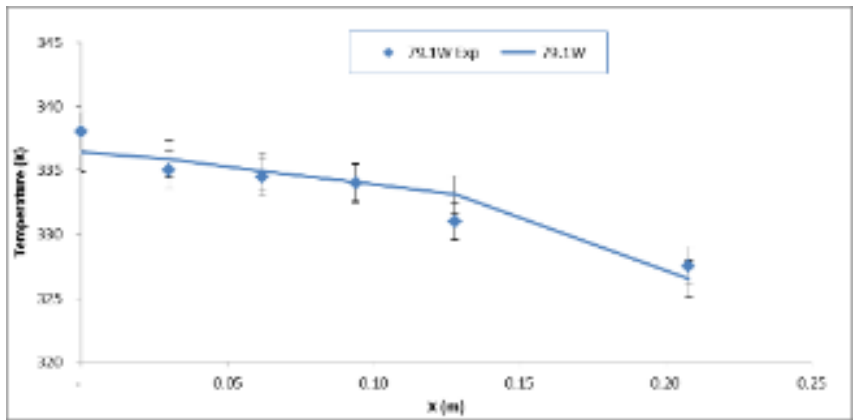

(a)

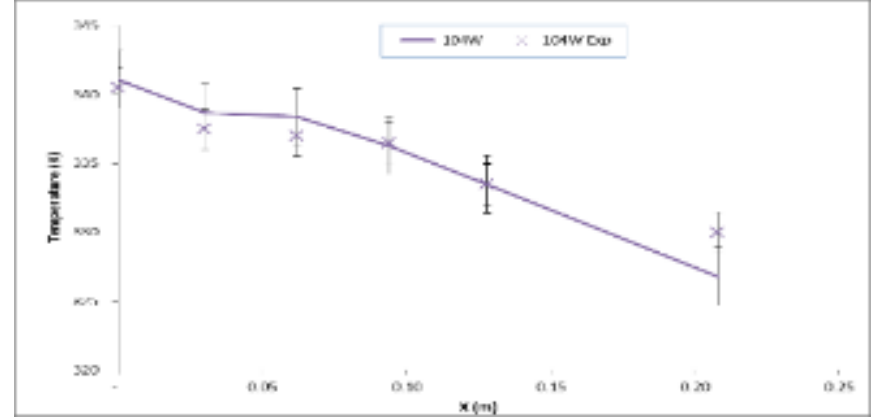

(b)

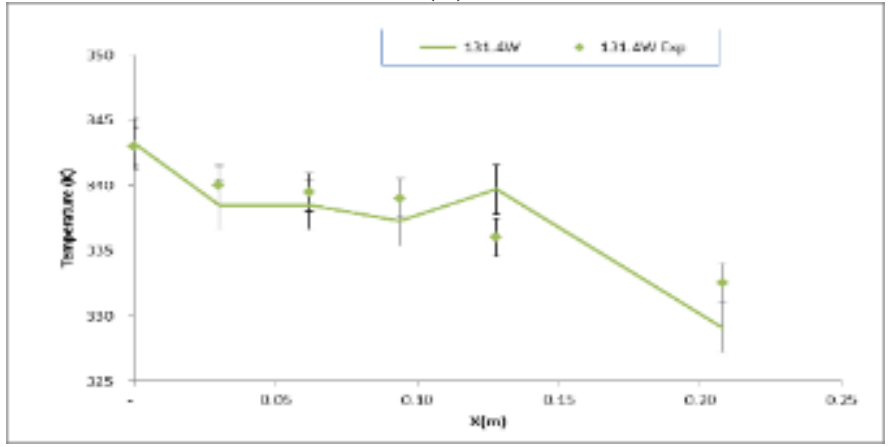

(c)

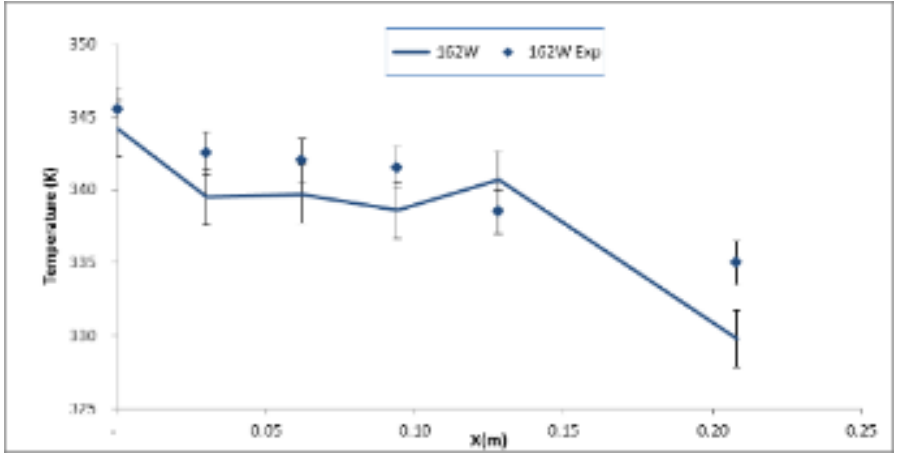

(d)

Figure 4. 48: Comparison of numerical and experimental temperature distributions along the heat loop at different heat inputs for a coolant inlet temperature of $338^{\circ} \mathrm{C}$ 
Inner Wall Temperature

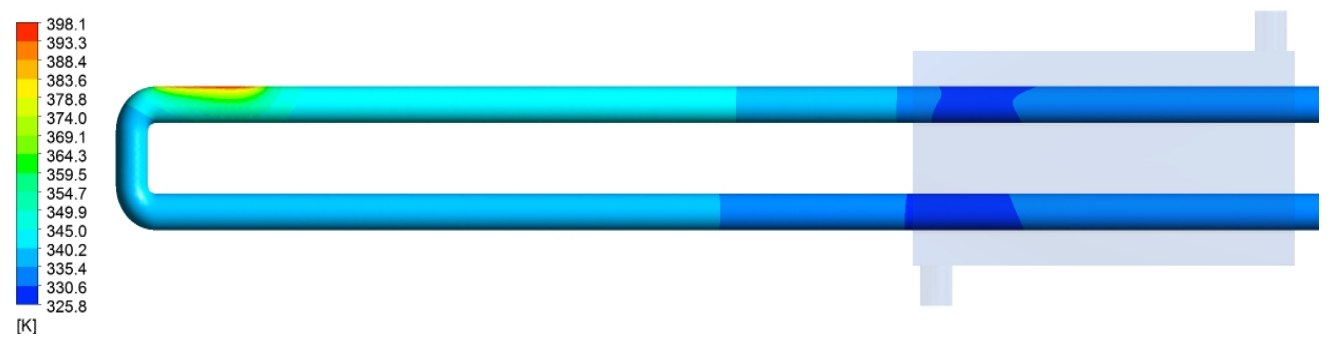

(a)

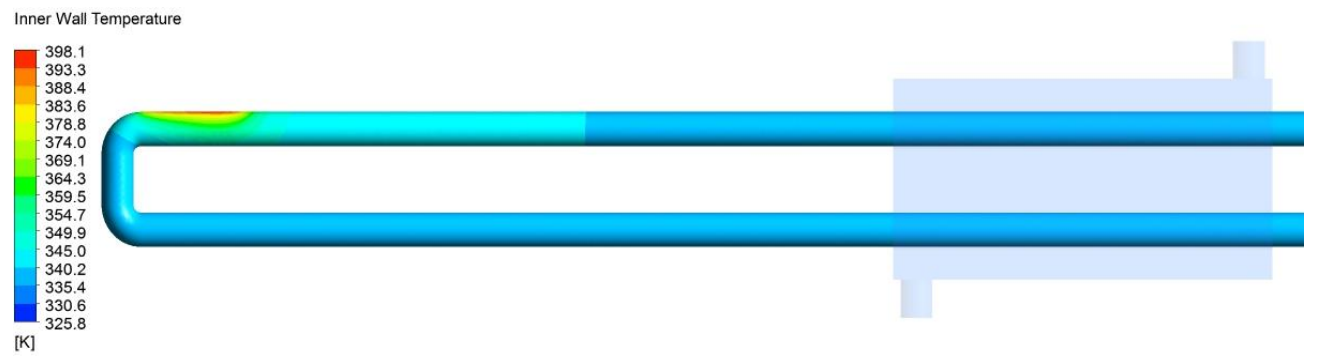

(b)

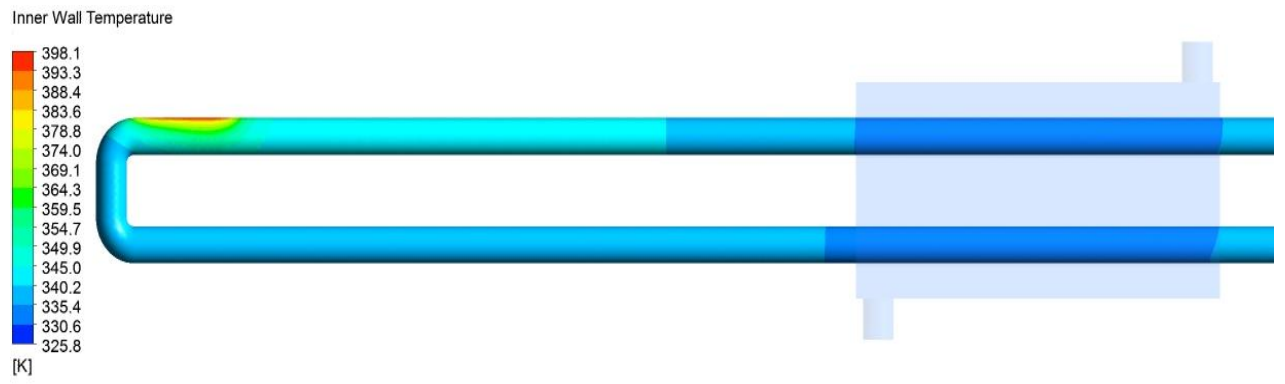

(c)

Figure 4. 49: Temperature contours of the working fluid for (a) $313 \mathrm{~K}$ condenser with $166 \mathrm{~W}$ at $715.4 \mathrm{~s}$, (b) $338 \mathrm{~K}$ condenser with $162 \mathrm{~W}$ at $474.8 \mathrm{~s}$, and (c) $323 \mathrm{~K}$ condenser with $171 \mathrm{~W}$ at $624.8 \mathrm{~s}$. 


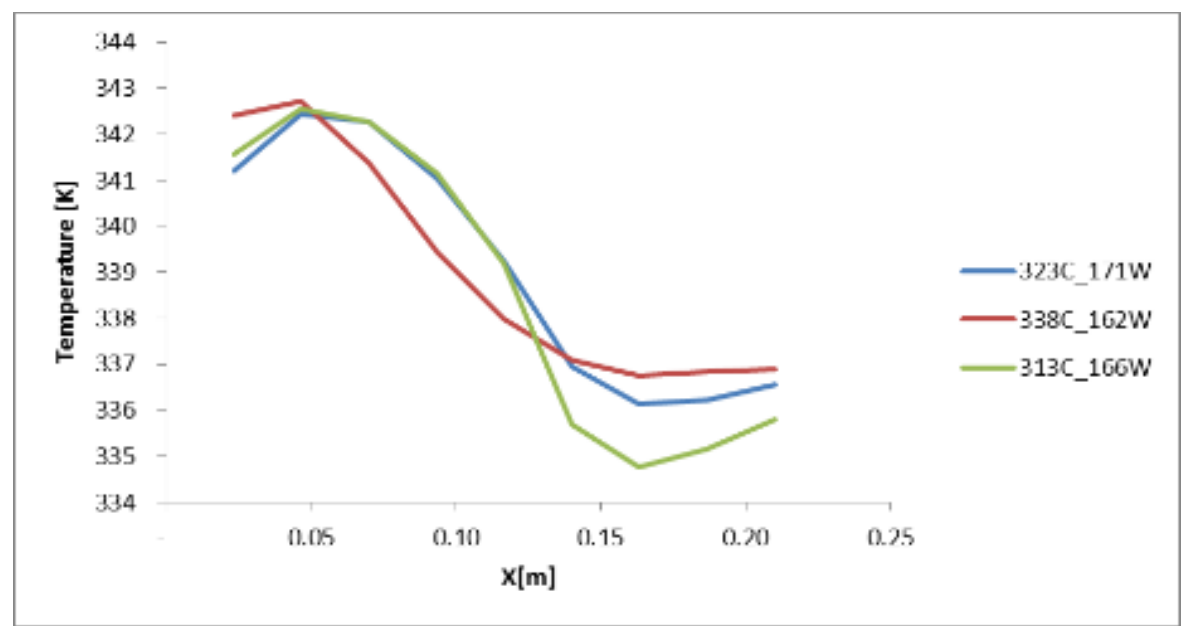

Figure 4. 50: Plot of fluid temperature vs. distance from the heater location 


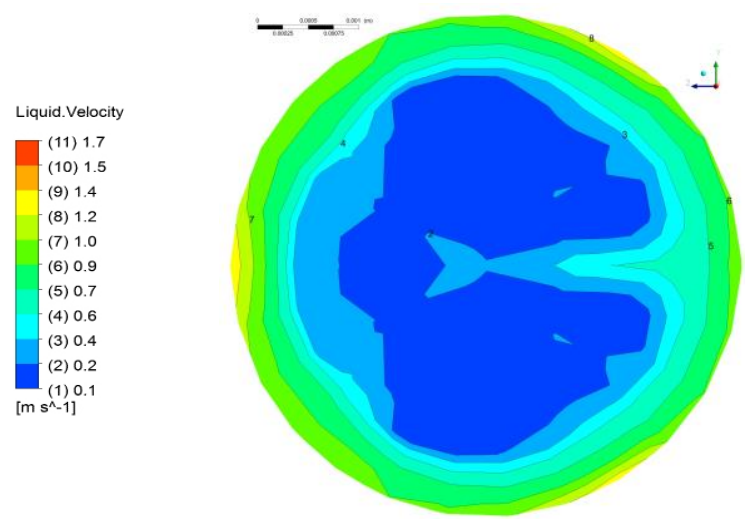

(a)

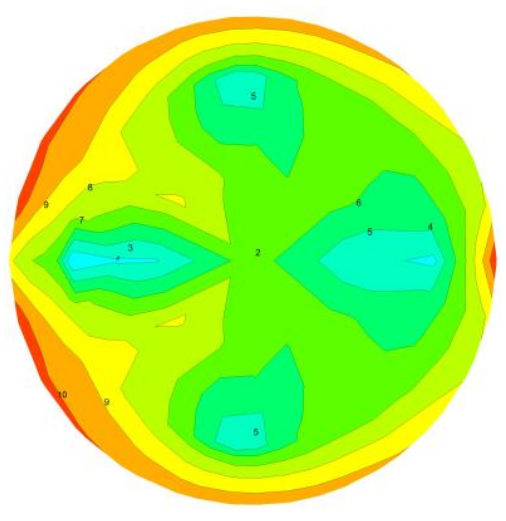

(b)

Liquid Velocity
\begin{tabular}{|l} 
(11) 0.9 \\
(10) 0.8 \\
(9) 0.7 \\
(8) 0.7 \\
(7) 0.6 \\
(6) 0.5 \\
(5) 0.4 \\
(4) 0.3 \\
(3) 0.3 \\
(2) 0.2 \\
(1) 0.1 \\
{$\left[\mathrm{~m} \mathrm{~s}^{\wedge}-1\right]$}
\end{tabular}

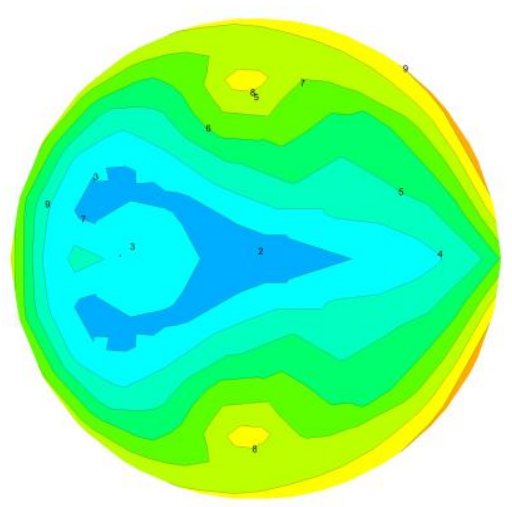

(c)

Figure 4. 51: Velocity profile of cooling liquid in the evaporator over Plane 2 with 313K Condenser and: (a) $166 \mathrm{~W}$ at $474.8 \mathrm{~s}$, (b) $166 \mathrm{~W}$ at $624.8 \mathrm{~s}$, and (c) $166 \mathrm{~W}$ at $715.4 \mathrm{~s}$ 
Figure 4.50 is a plot of the fluid temperature distributions along the heat loop at different condenser temperatures and heat inputs. Fig. 4.51 shows typical local velocity profiles of the fluid flow with different heat inputs. It was observed that the maximum velocity of the flow occurred near the wall rather than at the center of the pipe, as in the case of unidirectional steady flow, the so-called "annular effect" (T. S. Zhao and Cheng 1998b). The flow in the RMDHL is much more independent from the magnitude of the kinetic Reynolds number, the maximum axial velocity, and the phase difference between the pressure and the kinetic Reynolds number. It is understandable that a higher velocity near the wall will have the effect of promoting heat exchange between the wall and the fluid, which would explain why the RMDHL would have an enhanced heat transfer capacity. It was also observed that with an increased reciprocating frequency, the boundary layer near the wall becomes thinner, which further explains the heat transfer enhancement mechanism of the RMDHL. The standard deviation (SD) of the loop temperature about the mean was used as the measure of the temperature uniformity, the equation for the SD, Eq.(4.63), and the mean loop temperature, Eq. (4.64), are provided as follows:

$$
\begin{gathered}
\sigma_{T}=\sqrt{\frac{1}{N-1} \sum_{i=1}^{N}\left(T_{i}-T_{m}\right)^{2}} \\
\frac{1}{A} \int T d A=\frac{1}{A} \sum_{i=1}^{n} T_{i}\left|A_{i}\right|
\end{gathered}
$$

Fig. 4.52 show the temperature distributions over the RMDHL at heat inputs up to $171 \mathrm{~W}$ and a condenser inlet temperature of $323 \mathrm{~K}$. The figure indicates that although 
Inner Wall Temperature

(11)401.2
$(10) 391.4$
$(9) 381.8$
$(8) 372.4$
$(7) 363.3$
$(6) 354.4$
$(5) 345.7$
$(4) 337.2$
$(3) 328.9$
$(2) 320.9$
(1)313.0
$[K]$

(a)

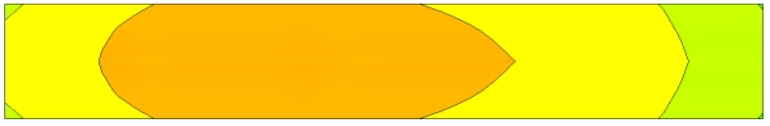

(b)

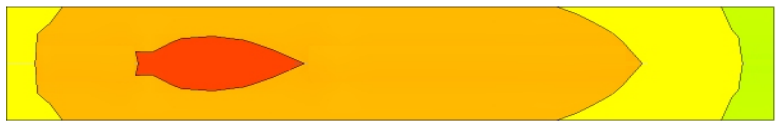

(c)

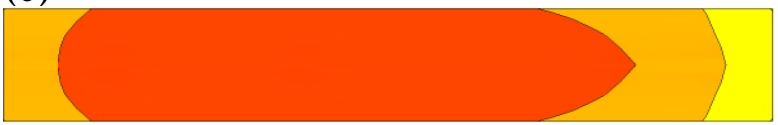

(d)

Figure 4. 52: Temperature contours of the heat loop showing surface temperature uniformity for $323 \mathrm{~K}$ condenser inlet temperature with heat flux at (a) $75.9 \mathrm{~W}$, (b) $126.9 \mathrm{~W}$, (c) $156 \mathrm{~W}$, and (d) $171 \mathrm{~W}$

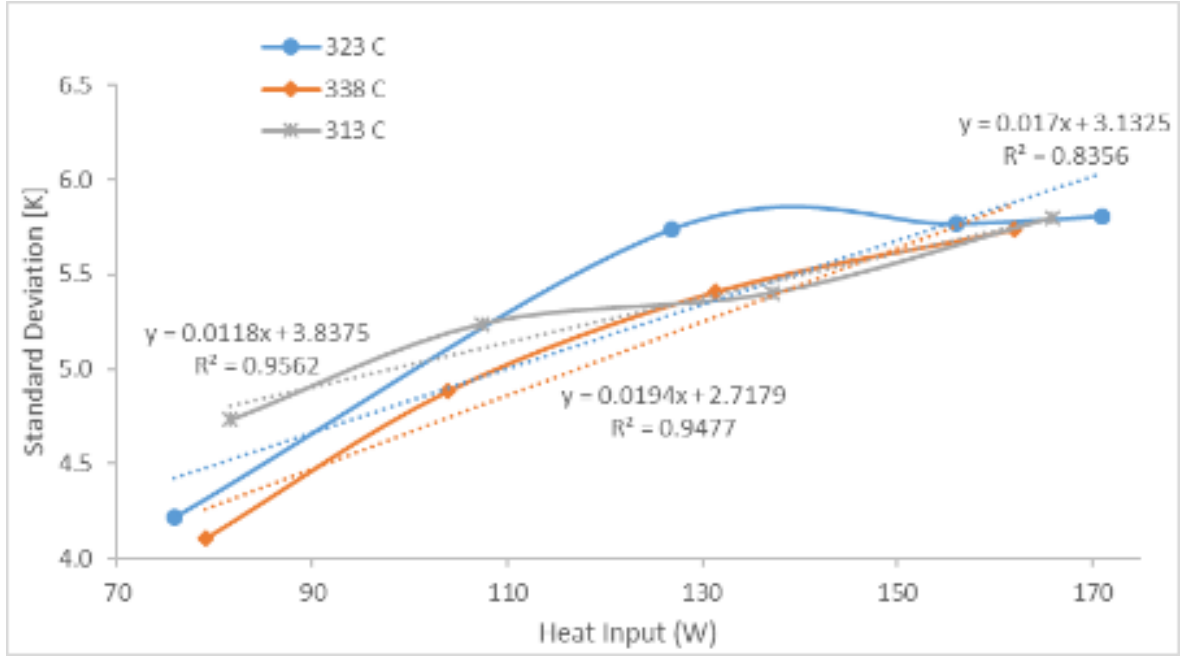

Figure 4. 53: Regression analysis for the SD of the of the cold plate static temperature for operating conditions 
the mean temperature across the surface varied with increasing heat input, the temperature level across the surface was similar. In some cases, up to $80 \%$ of the surface was maintained within a range of $1 \mathrm{~K}$. It can be seen that the mean temperatures along the heat loop increase with heat input, but the temperature distribution over the heat loop remains relatively uniform. To determine if this temperature uniformity was related to the condenser coolant temperature, a regression analysis of the SD for the various condenser coolant temperature was conducted and the results are presented in Fig 4.53. The he SD initially increases with heat input and then begins to form a plateau. In general, the value of the SD is less than $6 \mathrm{~K}$. Considering the high heat flux level over $300 \mathrm{~W} / \mathrm{cm}^{2}$ in the evaporator, the level of the temperature uniformity over the heat loop is rather high.

\subsubsection{Estimating Work done in RMDHL loop using Two-phase model.}

In section 4.2, an analytical approximation has been presented for the estimation of the work done by the RMDHL. When the liquid enters the heating section, boiling occurs, and pressure increases significantly. As the volume increases, pressure takes a maximum and then starts to decrease. The $P-V$ diagram constructed from the numerical model may also provide an approximation of the work done on the working fluid by the RMDHL driver. A potential application of the numerical model may be for developing the $\mathrm{p}-\mathrm{v}$ diagram for the RMDHL. The cycle efficiency of the RMDHL cycle could also be obtained from the P-V diagram of the operational cycle (Moran and Shapiro 2004). 
In the numerical model, the inlet velocity was defined as an input variable while the outlet velocity is an output variable. Fig. 4.54 shows a plot of the inlet volume flow rate against the iteration for one cycle of the driver while Fig. 4.55 is the corresponding outlet volume flow rate. Apparent changes in velocity as a result of the vapor generated within the loop has been indicated in Fig. 4.55. Fig. 4.56 superimposes the inlet and outlet flow rates to reveals different stages of acceleration and deceleration in the flow. Fig 4.57 shows a plot of the volume integral of pressure within the loop. These pressure and flow rate profile show almost the same pattern for each cycle. The PV diagram as a result of this analysis is presented in Fig. 4.58 that was developed using the data reduction flowchart presented in Fig. 4.59.

\subsubsection{Transient Characteristic of Cold plate with Two-phase application of RMDHL}

The present study revealed the two-phase heat transfer enhancement mechanism due to oscillating boundary layers. A possible correlation for the rewetting on the surface may be approximated by the work of Antal et al.(Antal, Lahey, and Flaherty 1991; Kolev 2011) to obtain a correlation for the heat transfer coefficient. The alignment between the numerical model and the experimental analysis has shown that the pattern of the vapor bubble formation at the heated surface and subsequent bubble removal 


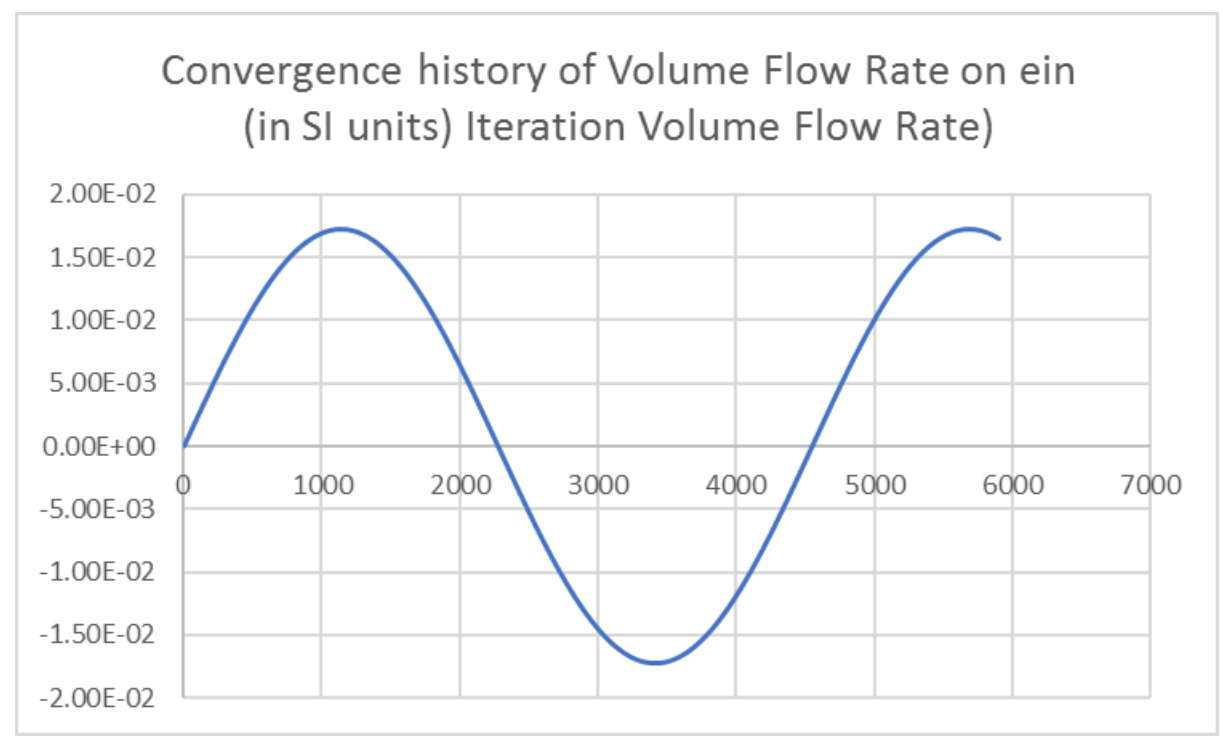

Figure 4. 54: Plot of Inlet volume flow rate against iteration

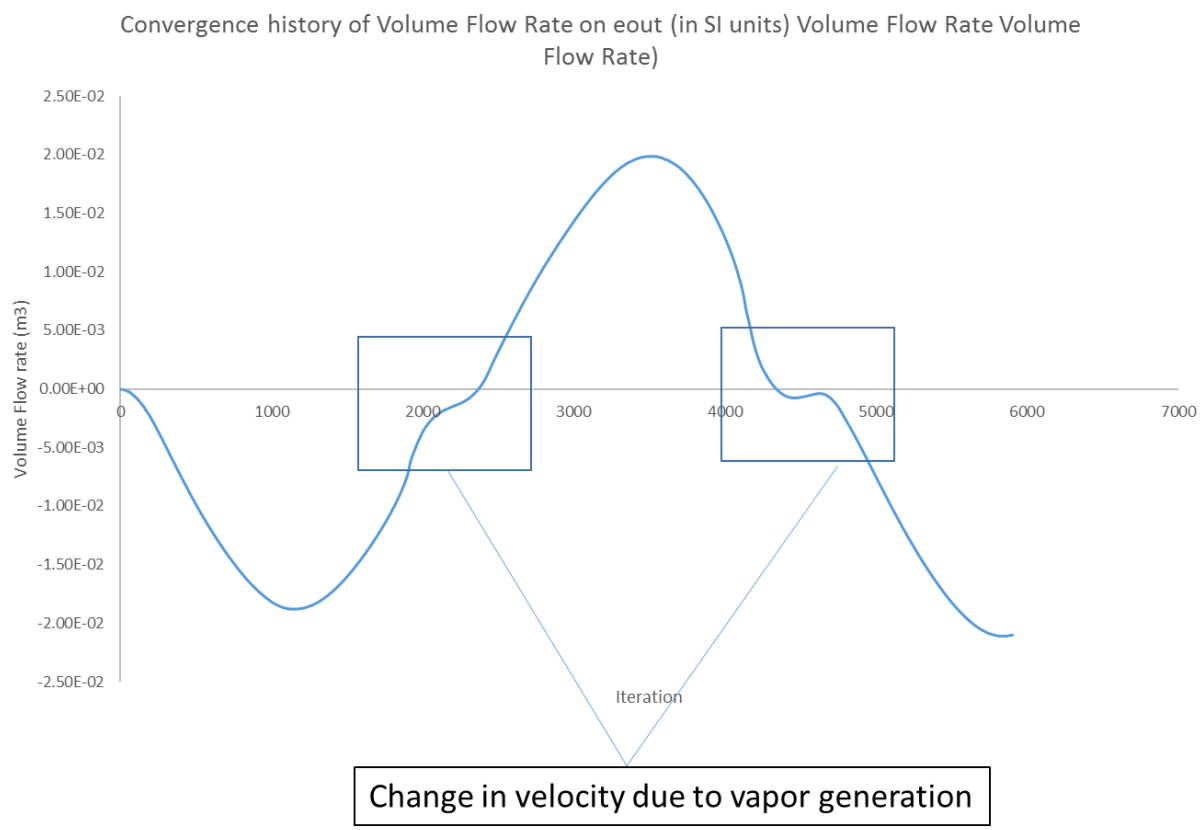

Figure 4. 55: Plot of Outlet volume flow rate against iteration 


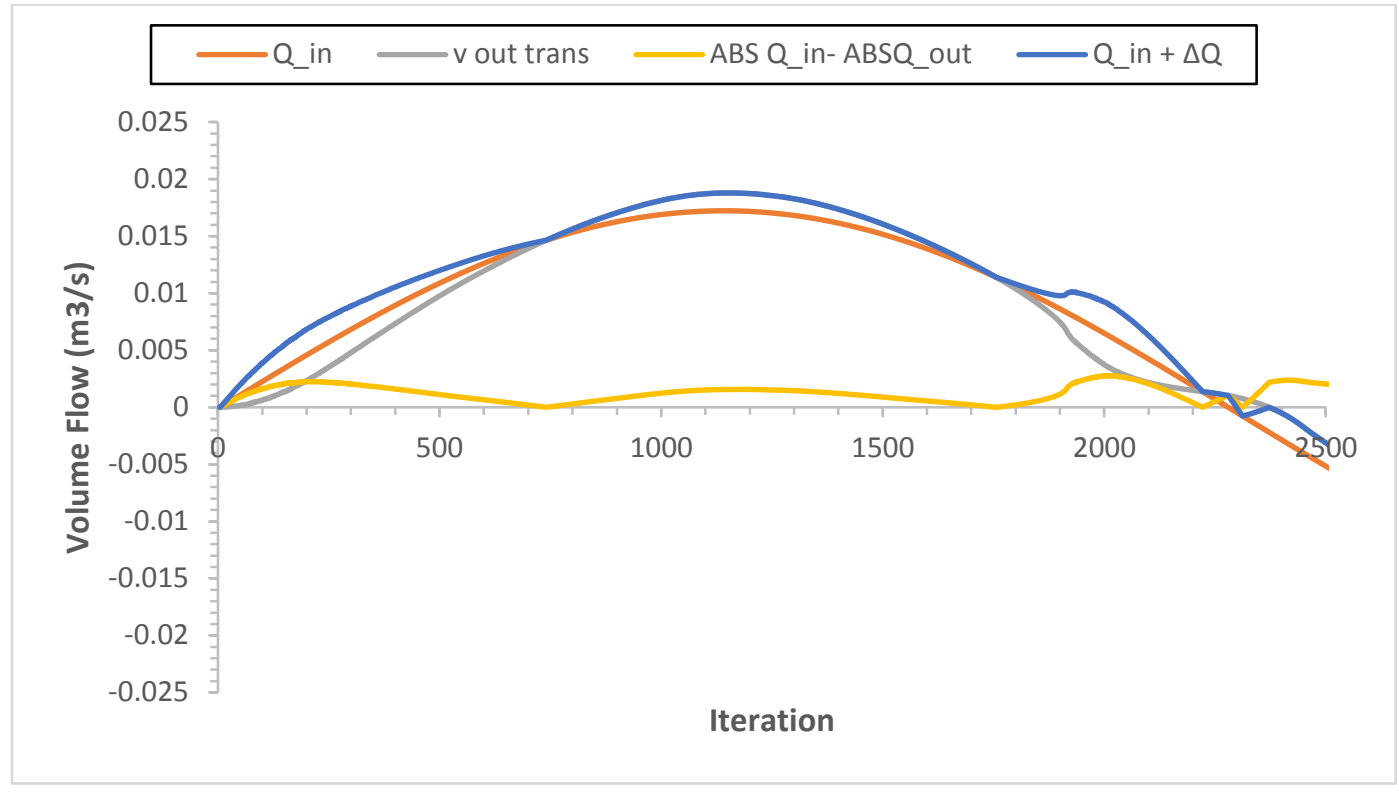

Figure 4. 56: Plot to compare the difference between inlet and out volume flow rate for half cycle of RMDHL driver to reflect variation in volume flow due to evaporation and condensation 


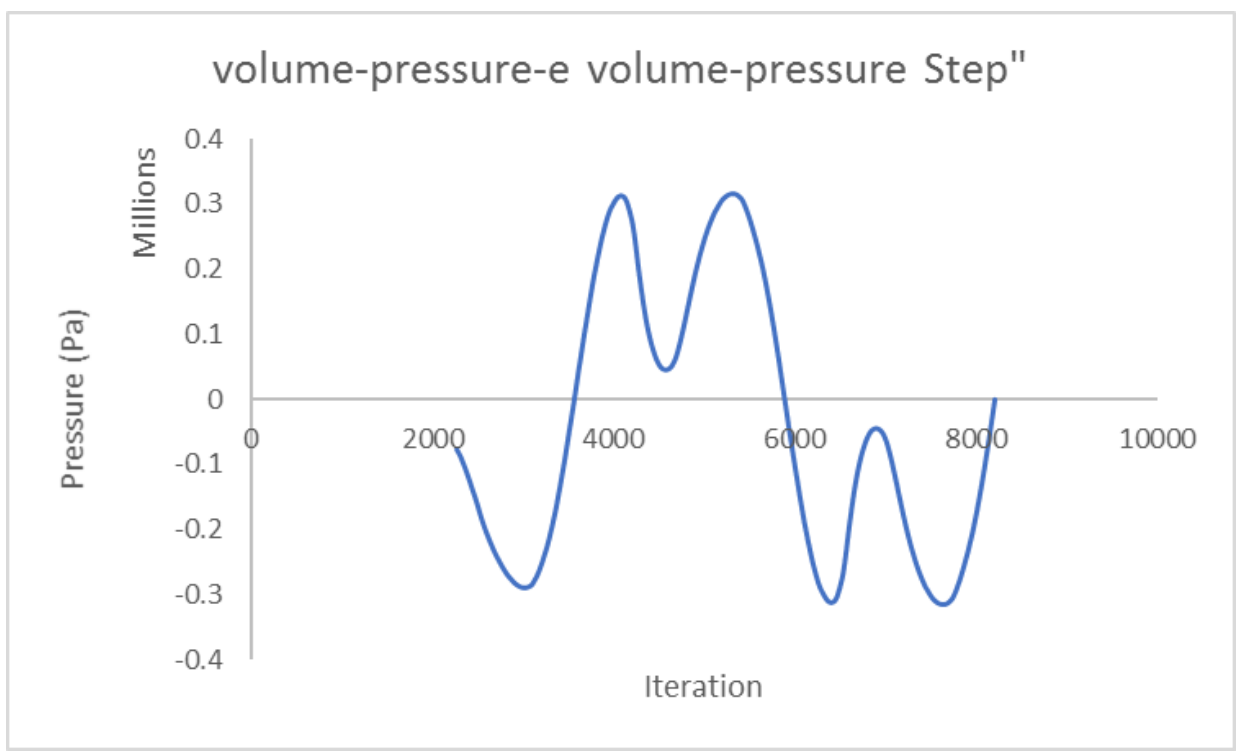

Figure 4. 57: Plot of Volume integral of pressure in the evaporator for one reciprocating cycle of the RMDHL driver

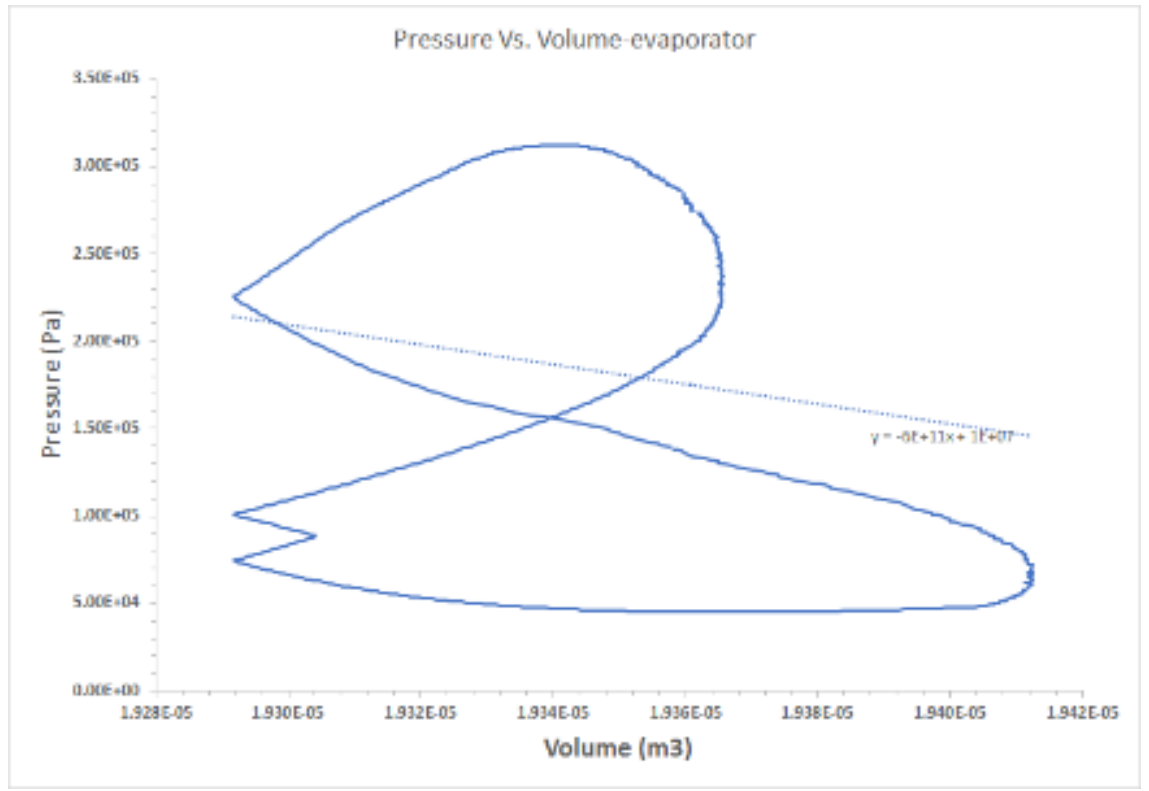

Figure 4. 58: P-V Diagram for RMDHL 


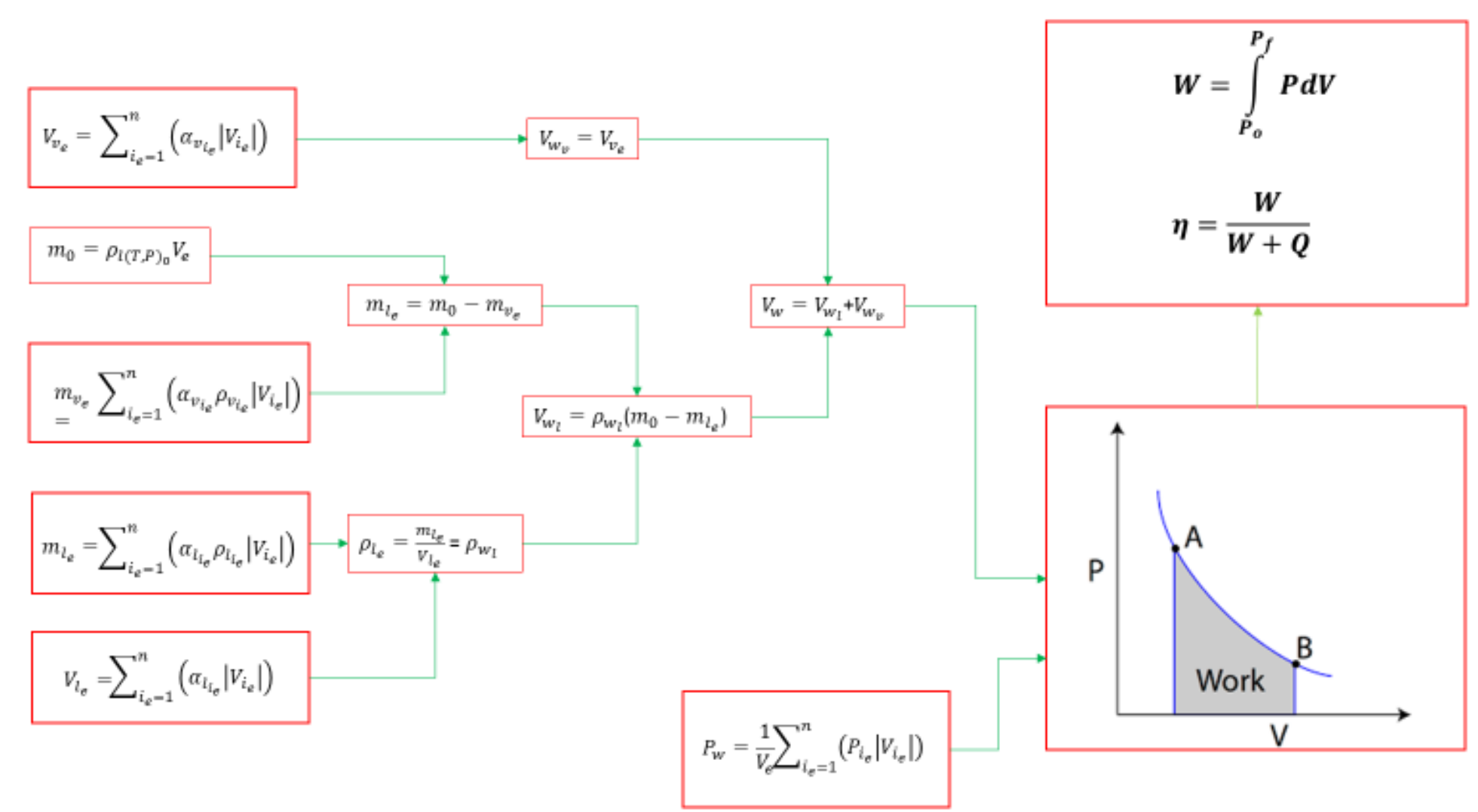

Figure 4. 59: Flow chart for developing P-V diagram from Numerical model 
from the surface can be predicted based on the work of Frank et al. (Frank, Shi, and Burns 2004), which is a modification of the Tomiyama lift model (Tomiyama 1998).

The experimental results of Cao and Gao (2003 and 2007) had suggested that for a two-phase reciprocating flow, however, this wall temperature fluctuation would diminish even under a relatively low reciprocating frequency, which concludes that the wall temperature fluctuation for the two-phase operation of the RMDL is associated with the heat input at the wall. Typical temperature variations across the hot plate at a high heat flux of $800 \mathrm{~W} / \mathrm{cm}^{2}$ is presented in Fig. 4.60 The temperature fluctuations increases with an increase in heat flux. The combination of the result of the higher heat transfer capacity of the two-phase flow and the oscillating velocity profile shows that the behavior of two-phase cooling does not follow the expectation that phase change will reduce temperature fluctuations significantly, although there exists a temperature stabilization regime..

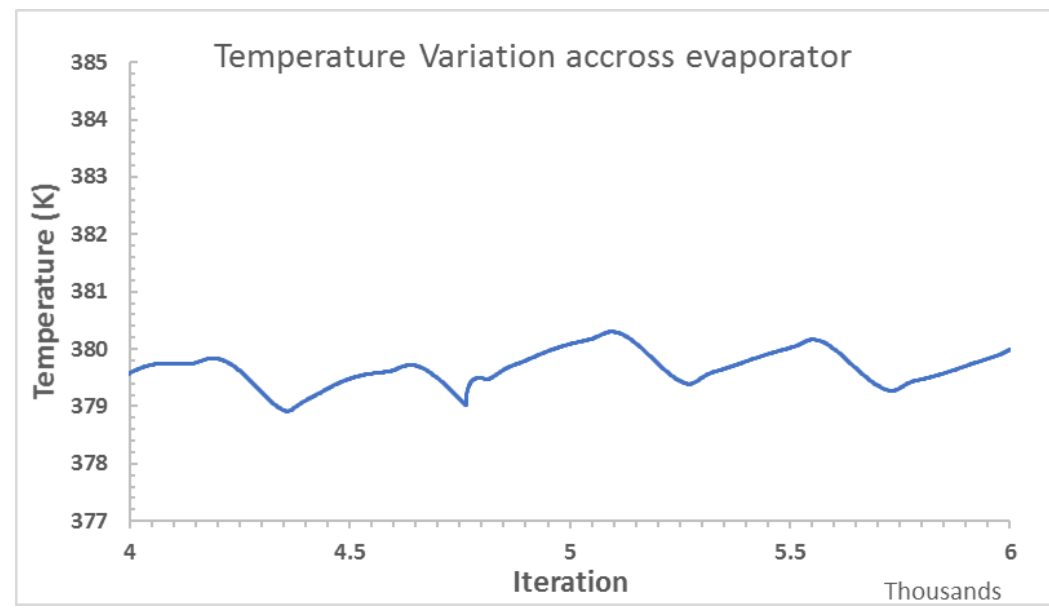

Figure 4. 60: Temperature fluctuations across hot plate at $600 \mathrm{~W} / \mathrm{cm}^{2}$ 
Typically, two-phase heat transfer system genetically tends to equalize the temperature in the system as is the case for other two-phase heat transfer devices such as a heat pipe and capillary pumped heat loop (Faghri, 1995; Peterson, 1994). The possible explanation for the fluctuation in Fig. 4.60 may be that the differences in vapor generation rate during the acceleration and deceleration of the flow within the loop may induce certain temperature fluctuation, although the fluctuation magnitude is rather small. If the input profile is changed from a sinusoidal form to a square wave form, the acceleration and deceleration phases could not be so pronounced. Future studies are required to investigate the relationship between the cold plate stabilization and the oscillating characteristic of pressure.

Theoretically, the wall temperature fluctuation may be eliminated due to the thin film evaporation and liquid rewetting at the wall in two-phase flows. In a pool boiling, the vapor bubbles generated at the heated surface would depart from the surface due to the buoyancy effect. In an internal two-phase heat transfer when the heat flux is sufficiently high, the generated bubbles would coalesce and form a vapor plug above the surface. Initially, there would exist a thin liquid film between the surface and the vapor plug, which would provide evaporative heat removal at the surface and support the growth of the vapor plug. However, numerical modeling in this study has shown that the RMDHL does not provide a timely removal of the vapor plug from the surface and subsequently liquid rewetting are essential to sustained heat removal at the surface before the depletion of the liquid film. 


\section{CHAPTER FIVE: ANALYTICAL STUDIES}

The investigation presented in the following section has been executed in order to provide empirical design and performance correlations for some of the other design parameters in addition to the thermal/fluid design consideration of the RMDHL in prior Chapters. These aspects may include pump selection, efficiency analysis, component selection and design, and ultimately understanding the limitations of the RMDHL. The approach adopted in this study is to tailor methodology developed in other similar (state of the art) studies or develop logical approximation from current studies to resolve some of the complexities associated with oscillating pipe flow. The procedure outlined in this section shows the design considerations for developing the prototypes used in this study and also outline some of the lessons learned in optimizing the RMDHL for thermal management. The aspects considered for the two phase operation of the loop include:

1. The power consumption of the actuator

2. Mechanism for the displacement of the working fluid

3. Working fluid (coolant) selection.

Consider a cooling loop under a two-phase working condition (liquid and vapor coexist) similar to those schematically shown in Fig. 5.1 to Fig 5.3. The driver as shown in Fig 5.1 is representative of a solenoid driver. An alternate configuration for 
the RMDHL including an actuator driver and a train of liquid plugs and vapor bubbles is illustrated Fig 5. 2. To illustrate the pressure distributions in a section of piping (Miyazaki 2000), one vapor bubble and one half of a liquid plug on the left and one half of a liquid plug on the right, have been shown in Fig. 5.3 where it was illustrated that the shear stress acting on the solid wall by the liquid plug is much higher than the shear stress induced by the vapor bubble. As shown in Fig. 5.2, When applying a heat source to the external circumference of the evaporator section, heat is transported by radial conduction through the evaporator wall and reaches the working fluid, resulting in vaporization. Also depicted in Fig 5.1 to Fig. 5.3, the cooling loop is assumed to have a condenser section on each side of the reciprocating driver, and the loop is symmetric about the line connecting the midpoints of the evaporator and reservoir. The analysis presented in this chapter will be based on the configuration shown in Fig 5.1 to Fig. 5.3.

\subsection{Working fluid consideration.}

One of the critical requirements of the present project is the temperature uniformity in the evaporator section (cold plate) where the chips are being cooled. For this purpose, a working fluid that exhibits a smaller value of $\mathrm{d} T / d p$ under a two-phase saturated condition should be considered. Additionally, the cold plate is required to work at a rather low temperature. Therefore, a working fluid having a low solidification temperature should be considered. Another requirement is to have a relatively low vapor pressure at the operation temperature of the cooling loop condenser for the consideration of the bellows design. 


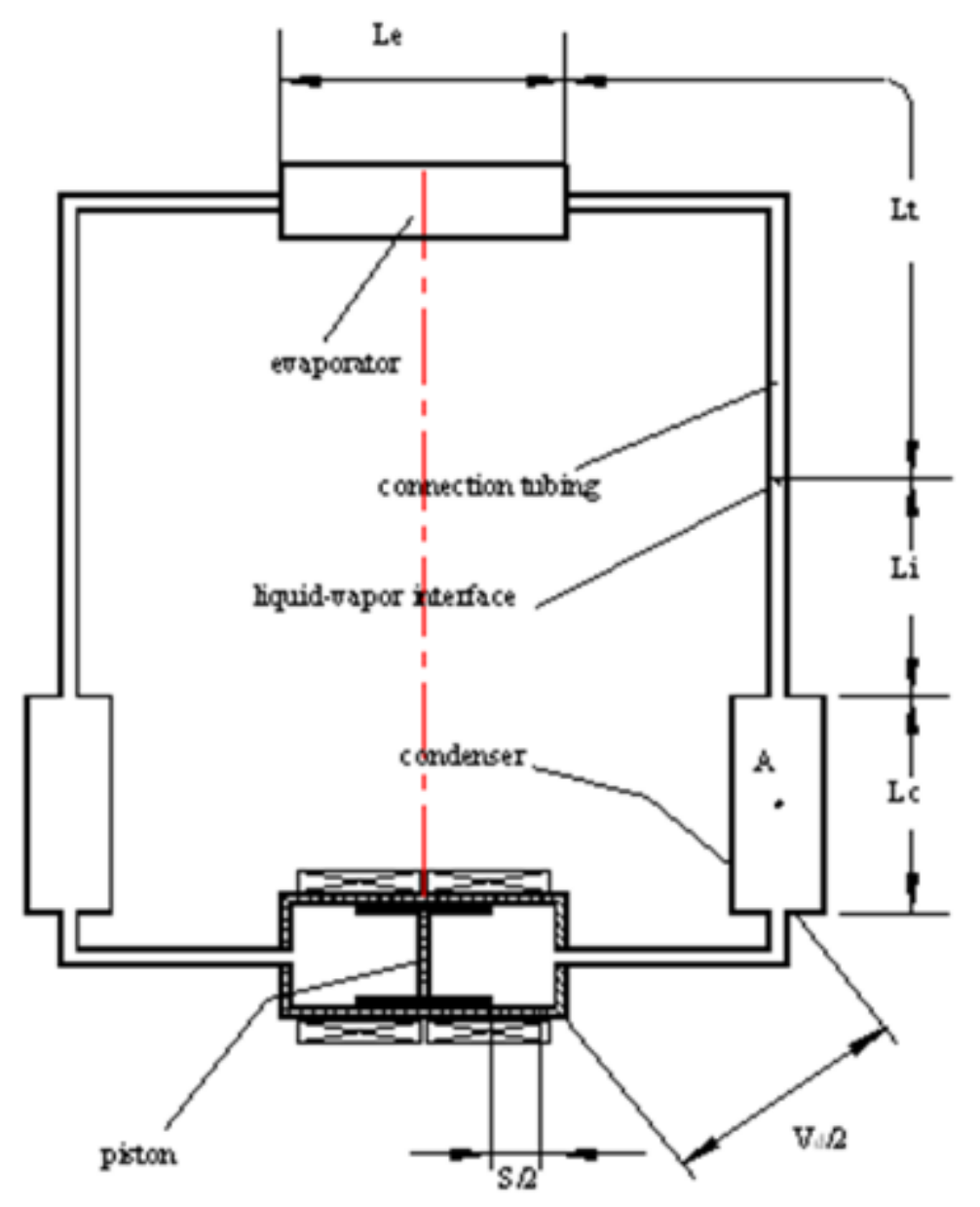

Figure 5. 1: The schematic of the heat loop (based on the solenoid type RMDHL) for the derivation of the working criterion 

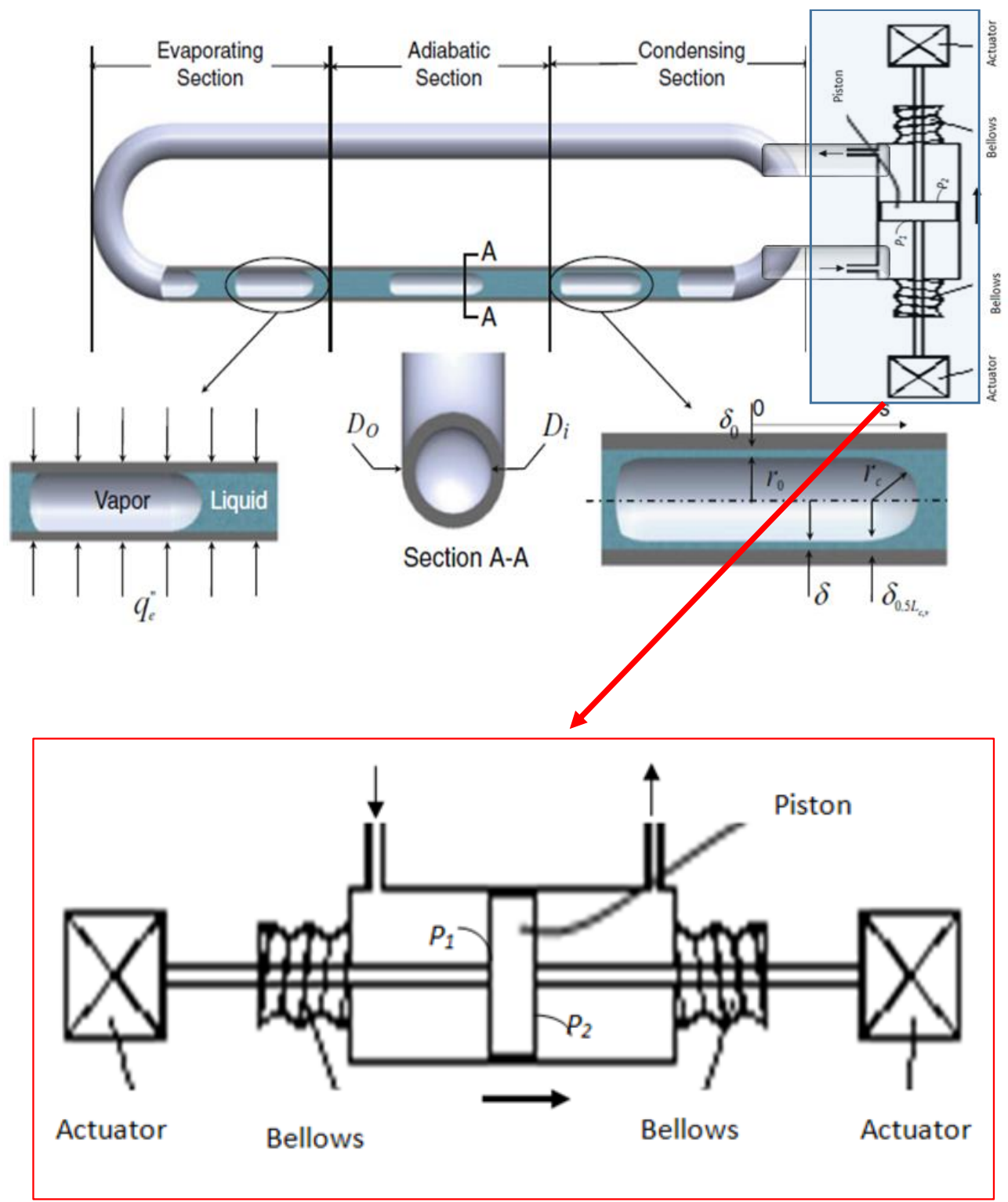

Figure 5. 2: Schematic drawing for Estimating power consumption in RMDHL illustrating the Motion of the piston in the reservoir. 


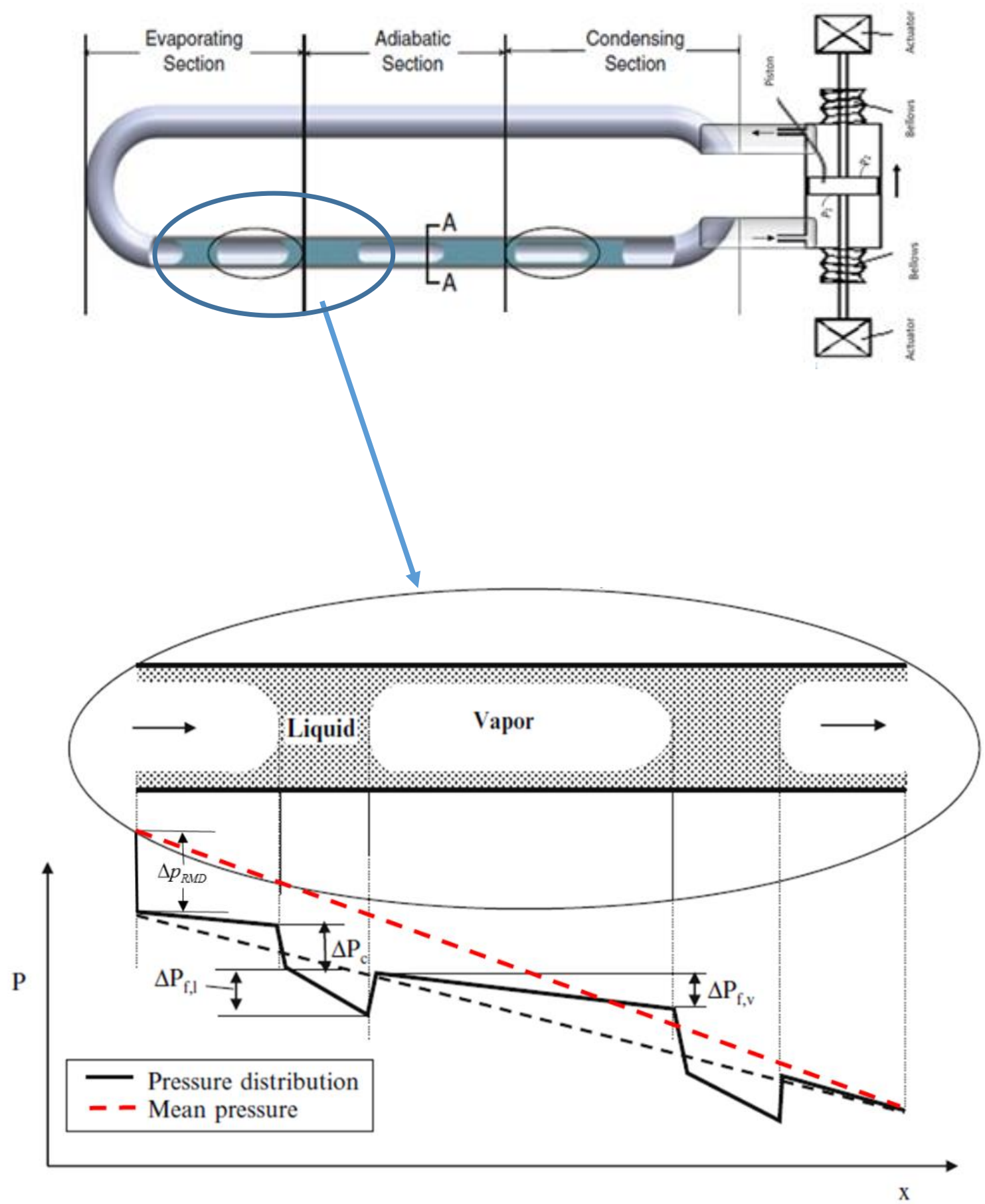

Figure 5. 3: Schematic of pressure distributions in a RMDHL configuration 


\subsection{Pump Selection and Power Analysis for the Oscillating Flow}

The selection of the prime mover in the RMDHL depends on the physical dimensions, working fluids, operating temperature, surface conditions, heat flux and total heat load, orientation, material, and the filling ratio, $\Phi$. The physical dimensions determine the critical fluid displacement. The fluid displacement within the loop in turn will be defined by the cooling requirement of the RMDHL and the fluid volume contained in the condenser and evaporator. This section presents a novel approach to determine the critical displacement of the reciprocating driver, inner surface/volume requirement in the condenser section and ultimately an approximation of the power consumption of a two-phase oscillating flow system using the RMDHL.

\subsubsection{Critical displacement of the reciprocating driver}

Using the method of (Cao and Gao 2008), the critical requirement for the operation of the cooling loop is given as:

$$
A_{p} S \geq 2\left(\frac{1}{2} A_{c} L_{c}+A_{t} L_{t}+\frac{1}{2} A_{e} L_{e}\right)
$$

The terms in the parentheses on the right-hand side of the above equation is the interior volume from the center of the condenser to the center of the evaporator on each side of the cooling loop, which reflects one of the essential geometric characteristics of the cooling loop in connection with the heat transfer distance and fluid displacement volume. If an effective displacement volume is defined for the entire cooling loop as follows: 


$$
V_{e f f}=2\left(\frac{1}{2} A_{c} L_{c}+A_{t} L_{t}+\frac{1}{2} A_{e} L_{e}\right)
$$

Eq. (5. 1) can be written as:

$$
A_{p} S \geq V_{\text {eff }}
$$

Eq. (5. 3) indicates that the liquid displacement volume of the piston as represented by $A_{p} S$ must be equal to or greater than the effective displacement volume of the cooling loop if the cooling loop is to work properly. Eq. (5. 3), is however, only true for a single-phase heat transfer mode. For a two-phase heat transfer mode, the criterion as represented by Eq. (5.2) or (5.3) is too conservative due to several reasons. Since the cross-sectional area of the reservoir is much greater than that of the rest of the cooling loop, the liquid velocity exiting the liquid reservoir should be relatively high. Even if after the piston has reached the dead center of the reservoir, the liquid would continue to move towards the evaporator until the kinetic energy associated with it is exhausted.

Additionally, once the liquid enters the evaporator section, some liquid will be evaporated into vapor. The evaporation will drastically change the volume of the flow stream and the liquid/vapor two-phase mixture will expand vigorously into the evaporator section. As a result, the section between the piston right dead center and the center of the evaporator in Fig. 5.1 would be filled with both liquid and vapor, and the flow is in a two-phase flow condition. It is understood that the liquid fraction would change substantially along the loop. For the derivation of a more concise relation, an 
effective liquid fraction, $\phi$, is used. By taking into account the two-phase flow condition, the liquid balance as represented by Eq. 3 should be modified as follows:

$$
A_{p} \frac{S}{2}+\frac{V_{d}}{2}+\varphi A_{c} L_{c}+\varphi A_{t} L_{i} \geq \frac{V_{d}}{2}+\varphi\left(A_{c} L_{c}+A_{t} L_{t}+\frac{1}{2} A_{e} L_{e}+\frac{1}{2} A_{c} L_{c}+A_{t} L_{i}\right)
$$

Following the same deriving procedure, the following relation is obtained:

$$
A_{p} S \geq 2\left(\frac{1}{2} A_{c} L_{c}+A_{t} L_{t}+\frac{1}{2} A_{e} L_{e}\right) \varphi
$$

or

$$
A_{p} S \geq \varphi V_{e f f}
$$

The value of $\phi$, by definition, is greater than zero and less than unity. An actual value of $\phi$, however, has to be determined experimentally for most practical applications due to the complex heat transfer process in the cooling loop. Still, Eq. (5.1) or Eq. (5.5) provides a concise criterion that could be used for the design of a heat loop. It should be pointed out that during the derivation of above relations, the liquid reservoir is assumed to contain pure liquid and the backflow through the gap between the outer surface of the piston and the inner surface of the reservoir casing is neglected. If the reservoir would deal with a two-phase liquid-vapor mixture and the back-flow effect is taken into account, the term $A_{p} S$ in the above equations may need to be multiplied by a driver efficiency $\eta$ that is less than unity:

$$
\eta A_{p} S \geq 2 \varphi\left(\frac{1}{2} A_{c} L_{c}+A_{t} L_{t}+\frac{1}{2} A_{e} L_{e}\right)
$$

The above equation may be rewritten in terms of the inner volumes of the condenser, tubing, and evaporator $\left(V_{c}, V_{t}\right.$, and $V_{e}$, respectively) as follows: 


$$
\eta A_{p} S \geq 2 \varphi\left(\frac{1}{2} V_{c}+V_{t}+\frac{1}{2} V_{e}\right)
$$

where the in nterior cross-sectional area of the evaporator are denoted by $L_{e}$ and $A_{e}$, respectively, the length and average interior cross-sectional area of the connection tubing between the evaporator and the condenser are $L_{t}$ and $A_{t}$, the length and interior cross-sectional area of each condenser section are $L_{c}$ and $A_{c}$, the interior volume of the section between the end of the condenser and the piston right dead center is $V_{d} / 2$, and the piston cross-sectional area and reciprocating stroke are $A_{p}$ and $S$, respectively.

\subsubsection{Inner surface/volume requirement in the condenser section}

The inner surface requirement may be translated into the internal volume requirement, $V_{c}$, which appears in Eq. (5.8). For a condenser section that involves heat transfer between the outer surface and the ambient, fins are typically required at the outer surface of the condenser. The overall heat transfer coefficient based on the outer surface area may be written as

$$
\frac{1}{U_{a}}=\frac{1}{h_{i}\left(A_{i} / A_{a}\right)}+A_{a} R_{w}+\frac{1}{\eta_{t, a} h_{a}}
$$

where $U_{a}$ is the overall heat transfer coefficient on the air side, $h_{i}$ is the heat transfer

coefficient associated with the working fluid inside the condenser, $A_{i}$ is the inner surface of the condenser, $A_{a}$ is the total heat transfer surface area in contact with air, $R_{w}$ is the thermal resistance related to the container walls, $\eta_{t, a}$ is the overall fin 
efficiency, and $h_{a}$ is the heat transfer coefficient on the air side. For a typical set of values as follows:

$$
h_{i}=5000 \frac{W}{m^{2} \cdot K}, A_{i} / A_{a}=14, \eta_{t, a}=0.90, h_{a}=100 \frac{W}{m^{2} \cdot K}
$$

Moreover, with negligible wall thermal resistance and an average condensation temperature of $T_{s}$, the following relations are obtained:

$$
\begin{aligned}
& U_{a}=80 \frac{W}{m^{2} \cdot K} \\
& Q=U_{a} A_{a}\left(T_{s}-T_{a}\right)=U_{a}\left(A_{i} / 0.14\right) \Delta T \\
& \rightarrow A_{i}=Q \times 0.14 /\left(U_{a} \Delta T\right)
\end{aligned}
$$

For $\Delta T=20^{\circ} C$ and $Q=(2400 / 2) W, A_{i}=0.21 m^{2}$

Therefore,

$$
V_{c}=\frac{\pi}{4} d_{c}^{2} L_{e f f, c}=\frac{\pi d_{c} L_{e f f, c}}{1} \frac{d_{c}}{4}=A_{i} \frac{d_{c}}{4}
$$

If the condenser tubing inner diameter, $d_{c}$, is taken to be $6 \mathrm{~mm}$, $V_{c}=1.575 \times 10^{-4} \mathrm{~m}^{3}$. It should be mentioned that the volume as calculated above is the condenser volume on each side of the cooling loop. Also, the above sample calculation is not a detailed design of the condenser; the purpose herein is to evaluate the volume requirement. In the first prototype demonstration, tap water will be used to remove heat from the condenser and therefore, detailed information on the condenser is not required at the present time. 


\subsubsection{Interior volume requirement associated with the evaporator}

Based on the configuration of the cold plate the interior volume associated with the evaporator $V_{e}=7.2 \times 10^{-5} \mathrm{~m}^{3}$

\subsubsection{Volume associated with the connection tubing}

$$
V_{t}=\frac{\pi}{4} d_{t}^{2} L_{t}
$$

where $d_{t}$ is the inner diameter of the tubing and $L_{t}$ is the length of the tubing on each side of the heat loop. If $d_{t}$ is taken as $0.012 \mathrm{~m}$ and $L_{t}$ is $0.5 \mathrm{~m}$ :

$$
V_{t}=5.65 \times 10^{-5} \mathrm{~m}^{3}
$$

\subsubsection{Liquid displacement volume in the reservoir}

$$
A_{p} S=\frac{\pi}{4} D_{r}^{2} S
$$

where $D_{r}$ is the inner diameter of the reservoir and $S$ is the stroke of the piston. If $D_{r}$ is taken as $0.08 \mathrm{~m}$ and $S$ is taken as $0.06 \mathrm{~m}$ :

$$
A_{p} S=\frac{\pi}{4} D_{r}^{2} S=3.0 \times 10^{-4} \mathrm{~m}^{3}
$$

\subsubsection{Overall balance requirement}

Finally, the sample design consideration in connection with the volume requirement of Eq. (5. 10) is checked:

$$
\eta A_{p} S=\eta \frac{\pi}{4} D_{r}^{2} S \geq 2 \varphi\left(\frac{1}{2} V_{c}+V_{t}+\frac{1}{2} V_{e}\right)
$$




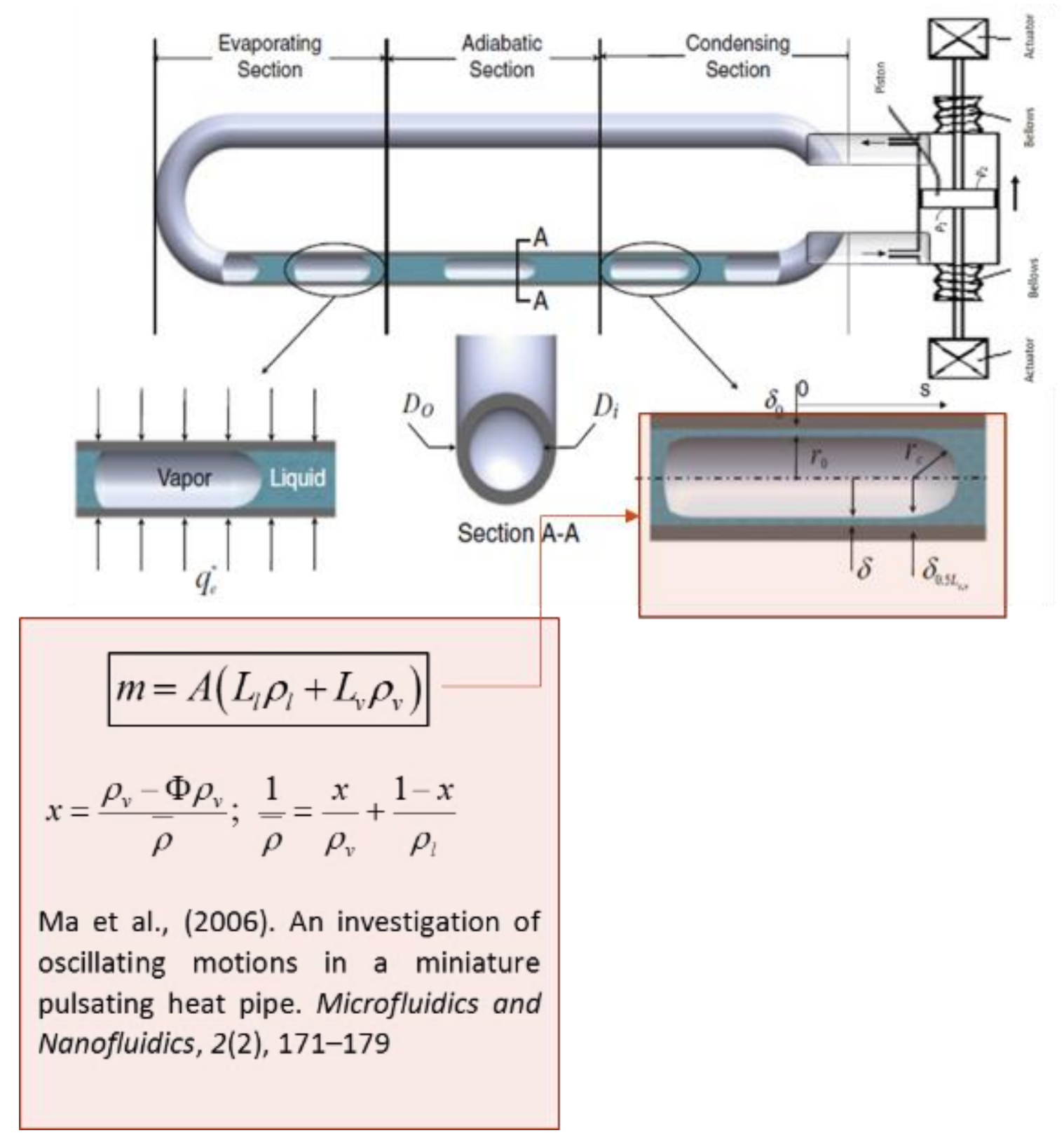

Figure 5. 4: Analysis of the volume of one vapor bubble formed during heat transfer in the RMDHL 
If $\eta$ is taken as 0.9 , and $\varphi$ is taken as 0.7 ,

$$
\begin{gathered}
\eta \frac{\pi}{4} D_{r}^{2} S=2.70 \times 10^{-4} m^{3}>2 \varphi\left(\frac{1}{2} V_{c}+V_{t}+\frac{1}{2} V_{e}\right)=2 \times 0.7 \times\left(\frac{1}{2} \times+1.575 \times 10^{-4}+\right. \\
\left.5.65 \times 10^{-5}+\frac{1}{2} \times 7.2 \times 10^{-5}\right) \mathrm{m}^{3}=2.40 \times 10^{-4} \mathrm{~m}^{3}
\end{gathered}
$$

above sample result indicates that the displacement volume requirement is satisfied.

\subsubsection{Power consumption of the actuator}

\subsubsection{Modeling and theoretical considerations}

A sufficient amount of energy must be provided to overcome the frictional losses associated with the motion of the working fluid to facilitate the reciprocating motion of the working fluid in the loop. The energy required is supplied by the action of a piston driven by an actuator. Fig 2 shows the motion of the piston schematically during a stroke from the left to the right of the reservoir. During this stroke, the actuator must overcome the pressure force difference between the frontal and back surfaces of the piston. The power consumption of the actuator or actuators may be calculated by the following relation:

$$
P=\left(p_{2}-p_{1}\right) A_{p} V_{p}=\Delta p A_{p} V_{p}
$$

The actual volume $V$ of the vapor bubble and liquid plug can be determined experimentally via a time-based analysis of high-speed camera footage of the operation of the working fluid and the construction of the RMDHL using transparent tubing material to capture the shape of the of the bubble as depicted in Fig 5.4.The liquid 
filling ratio $\Phi$ may be based on the "average density" of the working fluid defined by Ma and Chen (2006):

$$
\begin{aligned}
& x=\frac{\rho_{v}-\Phi \rho_{v}}{\bar{\rho}} \\
& \frac{1}{\bar{\rho}}=\frac{x}{\rho_{v}}+\frac{1-x}{\rho_{l}}
\end{aligned}
$$

where $p_{1}$ is the average pressure on the back face of the piston, $p_{2}$ is the pressure on the frontal face of the piston, $A_{p}$ is the cross-sectional area of the piston, and $V_{p}$ is the velocity of the piston.

To simplify the problem, the concept of the mean effective velocity, similar to that in an internal combustion engine analysis, is employed. The mean effective velocity, $\bar{V}_{p}$, is directly related to the piston reciprocating frequency, $F$, by the following relation:

$$
\bar{V}_{p}=2 S /(1 / F)
$$

By the definition of the mean effective velocity, the concept of the mean effective pressure difference is also defined in Eq. (5.15) for the power consumption of the actuator. The pressure drop as illustrated in Fig 5.3 across the piston is also the pressure drop of the working fluid and may be calculated by (Barreno et al. 2015):

$$
\left(\frac{\Delta p}{\Delta L}\right)=\frac{\mu U_{0}}{D^{2}}\left[\operatorname{Re}_{\omega} \cos (\omega t)+\left(32+f_{1}\right)\left(\frac{120}{L / D}\right)^{0.01} \sin (\omega t+\phi)\right]
$$

where: $f_{i}=0.19416 \operatorname{Re}_{\omega}-0.000575 \operatorname{Re}_{\omega}^{1.75}$;

$$
\beta=\left(A_{0} \sqrt{\operatorname{Re}_{\omega}}\right)
$$




$$
\begin{aligned}
& \phi=\phi_{1} \frac{1}{2} \tan ^{-1} \theta\left(\frac{\operatorname{Re}_{\omega}}{32+1.67 \mathrm{Re}_{\omega}^{0.59}}\right) \\
& \phi=\frac{\phi_{1}}{650}\left(1000-A_{0} \sqrt{\operatorname{Re}_{\omega}}\right)
\end{aligned}
$$

\subsubsection{Mass flow rate induced by the motion of the piston}

In the present cooling loop design, the piston/reservoir diameter may be in the range of $50-100 \mathrm{~mm}$ and piston stroke in the range of $50-80 \mathrm{~mm}$. The piston reciprocating frequency may vary within a wide range. For the present two-phase application, the heat transfer is primarily due to phase change, and subsequently, the mass supply requirement to the cold plate is drastically reduced as compared to a single-phase flow. Therefore, the piston may operate at a low frequency. A low frequency will also reduce the power consumption of the actuator.

If the piston diameter is taken to be $80 \mathrm{~mm}$, the piston stroke to be $60 \mathrm{~mm}$ and the frequency to be $0.2 s^{-1}$, the piston speed according to Eq. (18) would be:

$$
\bar{V}_{p}=2 S /(1 / F)=2 \times 0.06 /(1 / 0.2)=0.024 \mathrm{~m} / \mathrm{s}
$$

When R-11 is used, the corresponding mass flow rate is

$$
\dot{m}=\rho_{l} A_{p} \bar{V}_{p}=1440 \times \frac{\pi}{4} 0.08^{2} \times 0.024=0.174 \mathrm{~kg} / \mathrm{s}
$$

The Reynolds number is based on the mixture density and viscosity:

$$
\operatorname{Re}=\frac{V D_{h} \rho}{\mu}, \quad \frac{1}{\rho}=\frac{x}{\rho_{v}}+\frac{1-x}{\rho_{l}}, \quad \mu=\frac{x}{\mu_{v}}+\frac{1-x}{\mu_{l}}
$$

For R-11 at $40^{\circ} \mathrm{C}, \quad \rho_{l}=1440 \mathrm{~kg} / \mathrm{m}^{3}, \rho_{v}=9.7 \mathrm{~kg} / \mathrm{m}^{3}$,

$$
\mu_{l}=3.75 \times 10^{-4} \mathrm{~N}-\mathrm{s} / \mathrm{m}^{2}, \mu_{v}=1.19 \times 10^{-5} \mathrm{~N}-\mathrm{s} / \mathrm{m}^{2}
$$




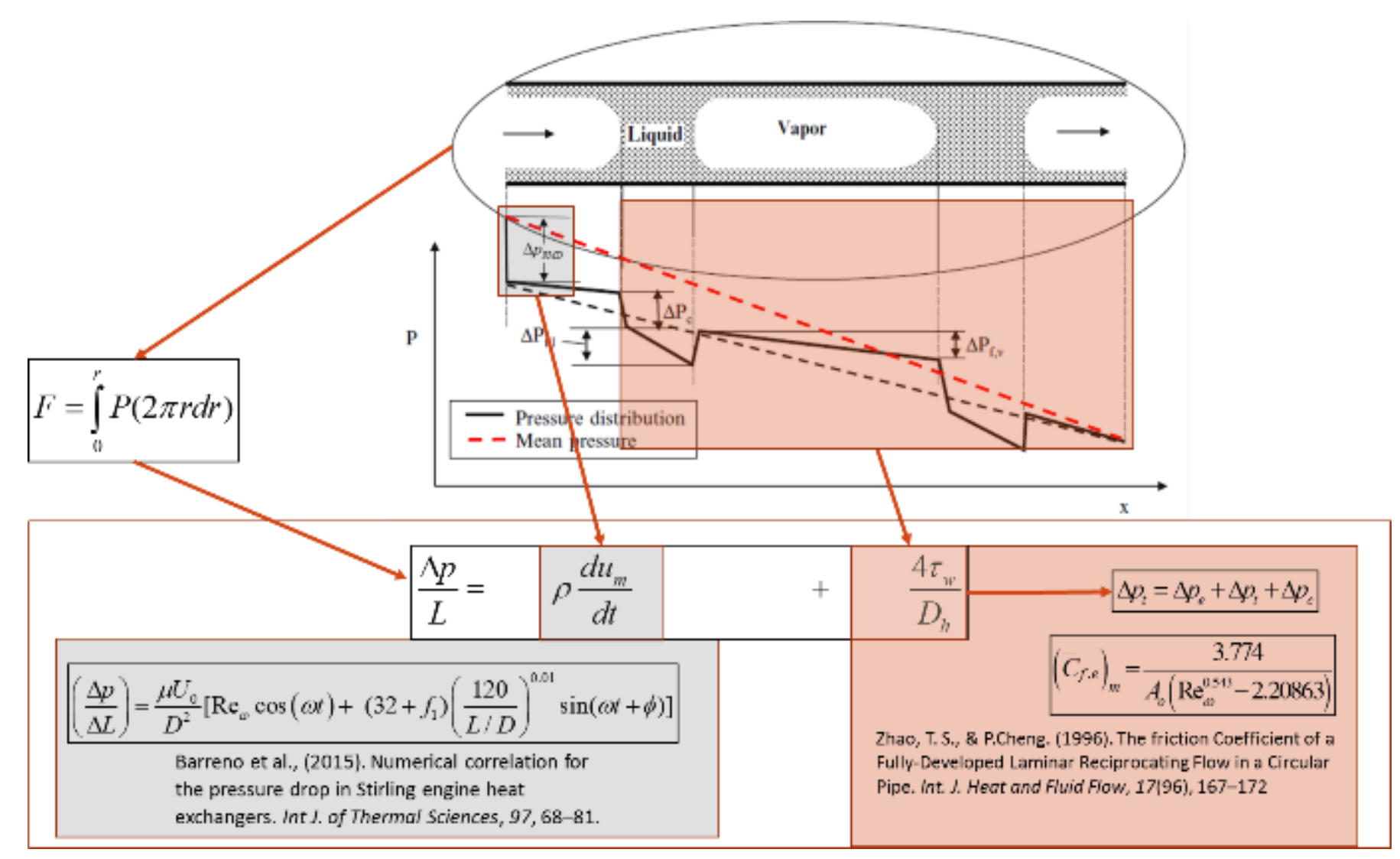

Figure 5. 5: Schematic illustration of how the Pressure forces in the RMDHL loop are resolved 


\subsubsection{Pressure drop in the evaporator section of the cooling loop}

In this exemplary calculation, the effective quality in the evaporator is taken to be $x=0.5$. A sufficiently low vapor quality is essential to keep the surface wall wetted and promote boiling heat transfer, which may be achieved once the requirement for liquid volume displacement is met. With the specified quality, the properties associated with the two-phase flow may be evaluated:

$$
\begin{gathered}
\rho=\left(\frac{0.5}{9.7}+\frac{1-0.5}{1440}\right)^{-1}=19.27 \mathrm{~kg} / \mathrm{m}^{3} \\
\mu=\left(\frac{0.5}{1.19 \times 10^{-5}}+\frac{1-0.5}{3.75 \times 10^{-4}}\right)^{-1}=2.31 \times 10^{-5} \mathrm{~N} \cdot \mathrm{s} / \mathrm{m}^{2} \\
\operatorname{Re}=\frac{V D_{h} \rho}{\mu}=\frac{4 \dot{m}}{\pi D_{h} \mu}=\frac{4 \dot{m}}{\pi \frac{2 w h}{(w+h)} \mu}=\frac{4 \times(0.174 / 6)}{\pi \times 0.0084 \times 2.31 \times 10^{-5}}=1.9 \times 10^{5}
\end{gathered}
$$

Therefore, through (T. S. Zhao and Cheng 1998a),

$$
\begin{aligned}
& \left(\bar{C}_{f, e}\right)_{m}=\frac{3.774}{A_{o}\left(\operatorname{Re}_{\omega}^{0.543}-2.20863\right)} \\
& c_{f}=0.0039, f=4 \times c_{f}=0.0158
\end{aligned}
$$

moreover, then the pressure drop across the cold plate is calculated:

$$
\Delta p=f\left(\frac{4}{\pi}\right)^{2} \frac{\dot{m}^{2} \Delta x}{2 D_{h}^{5} \rho}=0.0158 \times\left(\frac{4}{\pi}\right)^{2} \frac{(0.174 / 6)^{2} \times 0.2}{2 \times 0.0084^{5} \times 12.1}=4256.8 \mathrm{~Pa}
$$

\subsubsection{Pressure drop in the connection tubing of the cooling loop}

Since the size of the connection tubes is not subjected to serious limitations other than the displacement volume requirement, a larger diameter, $12 \mathrm{~mm}$, herein is used to 
reduce the pressure drop. In this calculation, a vapor quality of 0.5 is used to reflect the fact that at a given time one leg of the connection tube may be filled substantially with liquid, while the other leg may be filled substantially with vapor. The total connection tube length is taken to be $1 \mathrm{~m}$ with each leg being $0.5 \mathrm{~m}$.

$$
\begin{gathered}
\rho=\left(\frac{0.5}{9.7}+\frac{1-0.5}{1440}\right)^{-1}=19.27 \mathrm{~kg} / \mathrm{m}^{3} \\
\mu=\left(\frac{0.5}{1.19 \times 10^{-5}}+\frac{1-0.5}{3.75 \times 10^{-4}}\right)^{-1}=2.31=\times 10^{-5} \mathrm{~N} \cdot \mathrm{s} / \mathrm{m}^{2} \\
\operatorname{Re}=\frac{V D_{h} \rho}{\mu}=\frac{4 \dot{m}}{\pi D_{h} \mu}=\frac{4 \times(0.174)}{\pi \times 0.012 \times 2.31 \times 10^{-5}}=8.0 \times 10^{5}
\end{gathered}
$$

Therefore

$$
\begin{gathered}
c_{f}=0.003, f=4 \times c_{f}=0.012 \\
\Delta p=f\left(\frac{4}{\pi}\right)^{2} \frac{\dot{m}^{2} \Delta x}{2 D_{h}^{5} \rho}=0.012 \times\left(\frac{4}{\pi}\right)^{2} \frac{(0.174)^{2} \times 1.0}{2 \times 0.012^{5} \times 19.27}=61416 P a
\end{gathered}
$$

\subsubsection{Pressure drop in the condenser section of the heat loop}

For condenser design, an arrangement of 12 tubes having an inner diameter of $7 \mathrm{~mm}$ that are connected in parallel is considered. The tube length based on the total interior condenser volume as calculated before is $0.34 \mathrm{~m}$. The vapor quality is taken to be 0.2 , reflecting a much large percentage of liquid present in the condenser. Accordingly, 


$$
\begin{gathered}
\rho=\left(\frac{0.2}{9.7}+\frac{1-0.2}{1440}\right)^{-1}=47.23 \mathrm{~kg} / \mathrm{m}^{3} \\
\mu=\left(\frac{0.2}{1.19 \times 10^{-5}}+\frac{1-0.2}{3.75 \times 10^{-4}}\right)^{-1}=5.28 \times 10^{-5} \mathrm{~N} \cdot \mathrm{s} / \mathrm{m}^{2} \\
\operatorname{Re}=\frac{V D_{h} \rho}{\mu}=\frac{4 \dot{m}}{\pi D_{h} \mu}=\frac{4 \times(0.174 / 12)}{\pi \times 0.007 \times 5.28 \times 10^{-5}}=5.0 \times 10^{4}
\end{gathered}
$$

Therefore, $c_{f}=0.0052, f=4 \times c_{f}=0.0210$

$$
\Delta p=f\left(\frac{4}{\pi}\right)^{2} \frac{\dot{m}^{2} \Delta x}{2 D_{h}^{5} \rho}=0.021 \times\left(\frac{4}{\pi}\right)^{2} \frac{(0.174 / 12)^{2} \times 0.34}{2 \times 0.007^{5} \times 47.23}=1532.90 P a
$$

\subsubsection{Total pressure drop in the heat loop}

The total pressure drop in the cooling loop is the summation of individual pressure drops,

$$
\Delta p_{t}=\Delta p_{e}+\Delta p_{t}+\Delta p_{c}=4256.8+61416+1532.9=67205.7 P a
$$

The power consumption is:

$$
P=\Delta p_{t} A_{p} \bar{V}_{p}=67205.7 \times \frac{\pi}{4} 0.08^{2} \times 0.024=8.11 \mathrm{~W}
$$

The average force acting on the piston is

$$
F=\Delta p_{t} A_{p}=66855.5 \times \frac{\pi}{4} 0.08^{2}=337.8 \mathrm{~N}
$$




\subsection{Oscillating Motions and Vapor transport}

Heat removal rate at the condenser is coupled to a reasonable extent to the transport of mass and heat from the within the RMDHL loop. Oscillating motions with the RMDHL loop is defined using the template developed (Miyazaki 2000). Miyazaki developed the template to analyze transport of fluid within an oscillating heat pipe and assumes that heat transfer from the working from the wall to the working fluid results in the generation of vapor and slugs at the evaporator and the vapor and slug are the medium of heat transport.

Miyazaki (Miyazaki 2000) further assumed that the reciprocating motion is a result of pressure gradients developed due to pressure difference generated by vapor generation at the evaporator and condensation at the condenser. However, for the RMDHL, there is additional pressure gradient introduced by the reciprocating driver. This study modifies the results of Miyazaki (Miyazaki 2000) with the introduction of $\Delta p_{R M D}$, the replicating pressure from the RMDHL. The schematic for the analysis is presented in Fig 5.2 and Fig 5.6. The condensing and evaporating sections are connected by an adiabatic section and the portion of the RMDHL not required for the analysis is not shown. The following assumptions are made:

1. It is assumed that heat is transported by radial conduction from the heat source through the evaporator wall to reach the working fluid because the pipes are cylindrical. 
2. The heat transfer results in sensible heating and vaporization of the working fluid and vaporization cause a volume expansion in the working fluid; the expansion creates an increase in pressure in the working fluid.

3. Volume contraction due to the cooling occurs in the condenser.

4. The heat transfer in the evaporating section of the RMDHL is a combination of nucleate boiling and bulk convection at the macroscopic level.

5. Acceleration of the working fluid as a result of the oscillating flow is negligible.

6. The temperature difference between the evaporator and condenser has a cosine waveform (Miyazaki 2000) given by:

$$
\Delta T=\frac{\Delta T_{\max }-\Delta T_{\min }}{2}[1+\cos (\omega t)]
$$

7. The vapor phase is considered an ideal gas.

8. The average density of the working fluid is given as (Ma et al. 2006):

$$
x=\frac{\rho_{v}-\Phi \rho_{v}}{\bar{\rho}}
$$

9. Quality $x$ based on the filling ratio $\Phi$ is given as (Miyazaki 2000):

$$
\frac{1}{\bar{\rho}}=\frac{x}{\rho_{v}}+\frac{1-x}{\rho_{l}}
$$




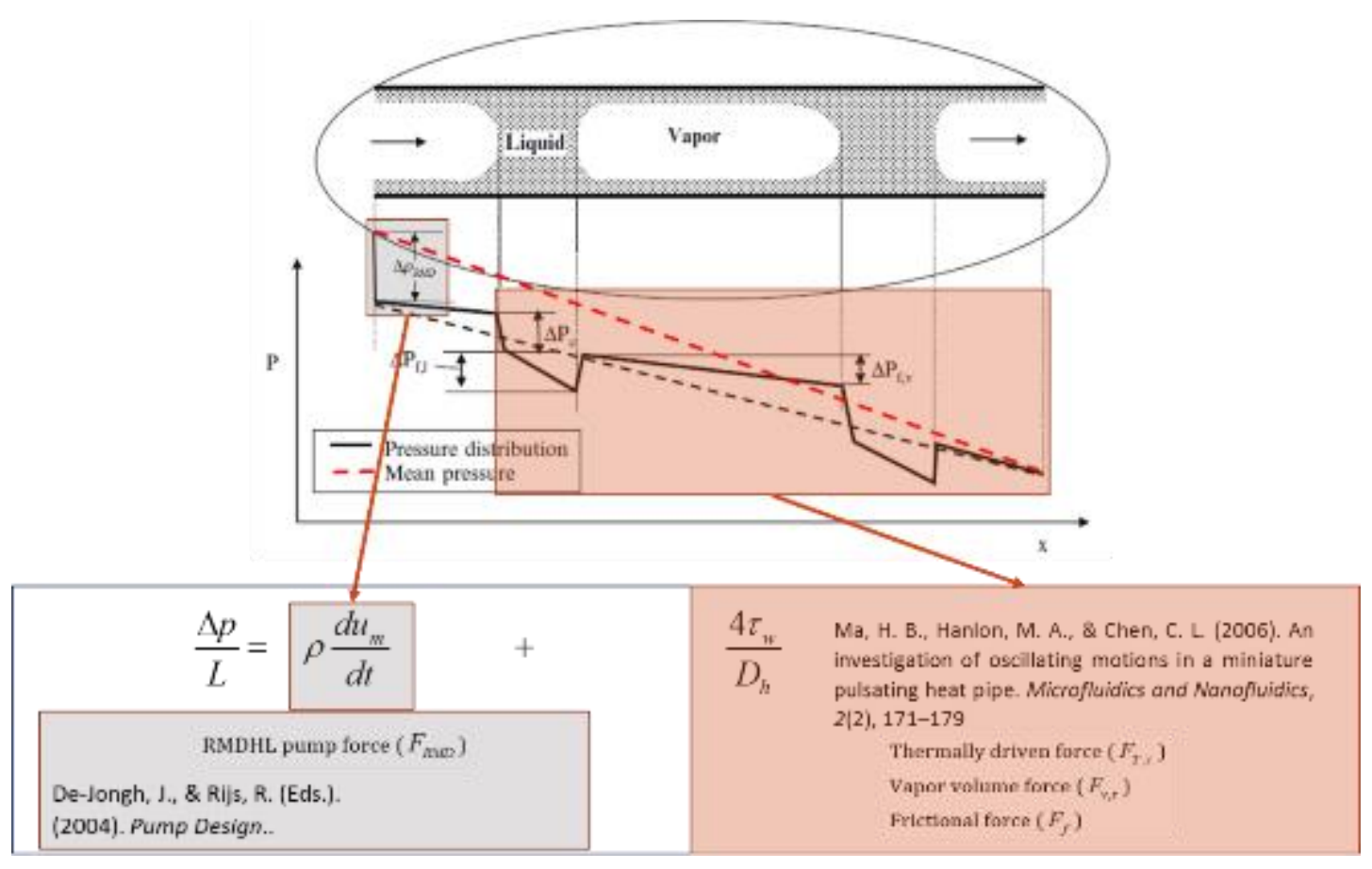

Figure 5. 6: Pressure distributions in the RMDHL adapted from Ma et al.(2006) 
10. The vapor temperature in the evaporator section is known, then the vapor pressure in the evaporating section can be estimated using the ClausiusClapeyron Equation and is given as

$$
p_{e}=p_{0} \exp \left[\frac{h_{f g}}{R} \frac{T_{e}-T_{0}}{T_{e} T_{0}}\right]
$$

11. Similarly, vapor pressure in the condenser is given as

$$
p_{e}=p_{0} \exp \left[\frac{h_{f g}}{R} \frac{T_{c}-T_{0}}{T_{c} T_{0}}\right]
$$

12. The summation of pressure gives the instantaneous Pressure difference in the RMDHL at the evaporator, condenser on the reciprocating driver

$$
\Delta p=p_{c}\left[\exp \left(\frac{h_{f g}}{R} \frac{T_{e}-T_{c}}{T_{e} T_{c}}\right)-1\right]
$$

Taylor series expansion of Eq. (25) gives

$$
\Delta p=\Delta T \frac{h_{f g} \rho_{v, c}}{T_{e}}
$$

The momentum equation for the case under consideration can be expressed as:

$$
\sum F=m a=m \frac{d^{2} x}{d \tau^{2}}=\left(L_{l} \rho_{l}+L_{v} \rho_{v}\right) A \frac{d^{2} x}{d \tau^{2}}
$$

where $L_{v}$ and $L_{l}$ are the total length for the vapor bubbles and liquid slugs, respectively 
An examining the oscillating phenomena occurring in the RMDHL, there should exist four kinds of forces:

1. the thermally driven force $F_{T, \tau}$

2. the vapor volume force $F_{v, \tau}$

3. the frictional force $F_{f}$

4. the RMDHL pump force $F_{R M D}$

For reciprocating flow is driven by an oscillating pressure gradient given by:

$$
\frac{1}{\rho} \frac{\partial p}{\partial x}=k \cos \omega \tau
$$

where $k$ and $\omega$ are the amplitude and circular frequency of the pressure gradient imposed by the reciprocatory driver. If Eq. (31) is multiplied by $2 \pi r d r$ and integrated over $r$ and $x$ (T. S. Zhao and P.Cheng 1996) it becomes:

$$
\frac{\Delta p}{L}=\rho \frac{d u_{m}}{d t}+\frac{4 \tau_{w}}{D_{h}}
$$

$u_{m}$ corresponds to the mean cross-sectional velocity from the RMDHL driver given as

$$
\begin{aligned}
& u_{m}=u_{\text {max }} \sin (\omega \tau) \\
F_{R M D}= & A \Delta p_{R M D}=A \omega u_{\max }\left(L_{l} \rho_{l}+L_{v} \rho_{v}\right) \cos (\omega \tau) \\
& \frac{4 \tau_{w}}{D_{h}} \text { corresponds to the frictional force } F_{f} \text { defined as (Ma, Hanlon, and Chen }
\end{aligned}
$$

2006), the vapor volume force $F_{v, \tau}$ (based on the assumption that the vapor is an ideal gas) is given by as: 


$$
\begin{aligned}
& F_{f}=A \Delta p_{f}=A\left[f_{l} \operatorname{Re}_{l}\left(\frac{\mu_{l} L_{l}}{2 D_{h}^{2}}\right)+f_{v} \operatorname{Re}_{v}\left(\frac{\mu_{v} L_{v}}{2 D_{h}^{2}}\right)\right]\left(\frac{d x}{d \tau}\right) \\
& F_{v, \tau}=A \Delta p_{v}=\frac{A \rho_{v} R T}{L_{v}} x
\end{aligned}
$$

where $\operatorname{Re}_{l}=\frac{\rho_{l} D}{\mu_{l}} \frac{d x}{d t}$ and $\operatorname{Re}_{v}=\frac{\rho_{v} D}{\mu_{v}} \frac{d x}{d t}$

The thermally driven force $F_{T, \tau}$ is attributed to the differential vapor pressure resulting from the difference in temperature between the condenser and the evaporator given as:

$$
F_{T, \tau}=A \Delta T \frac{h_{f g} \rho_{v, c}}{T_{e}}=A \frac{h_{f g} \rho_{v, c}}{T_{e}} \frac{\Delta T_{\max }-\Delta T_{\min }}{2}[1+\cos (\omega \tau)]
$$

Substituting Eq. (28) to Eq. (31) into Eq. (26):

$$
\begin{aligned}
&\left(L_{l} \rho_{l}+L_{v} \rho_{v}\right) A \frac{d^{2} x}{d \tau^{2}} \\
&+A \omega u_{\max }\left(L_{l} \rho_{l}+L_{v} \rho_{v}\right) \cos (\omega t) \\
&+A\left[f_{l} \operatorname{Re}_{l}\left(\frac{\mu_{l} L_{l}}{2 D_{h}^{2}}\right)+f_{v} \operatorname{Re}_{v}\left(\frac{\mu_{v} L_{v}}{2 D_{h}^{2}}\right)\right]\left(\frac{d x}{d \tau}\right) \\
&+\frac{A \rho_{v} R T}{L_{v}} x \\
& \quad=A \frac{h_{f g} \rho_{v, c}}{T_{e}} \frac{\Delta T_{\max }-\Delta T_{\min }}{2}[1+\cos (\omega \tau)]
\end{aligned}
$$

Eq. (32) is a mathematical model predicting the oscillation movement in the RMDHL. Which can be rewritten as: 


$$
\begin{aligned}
\left(L_{l} \rho_{l}+L_{v} \rho_{v}\right) A & \frac{d^{2} x}{d \tau^{2}} \\
+A\left[f_{l}\right. & \left.\operatorname{Re}_{l}\left(\frac{\mu_{l} L_{l}}{2 D_{h}^{2}}\right)+f_{v} \operatorname{Re}_{v}\left(\frac{\mu_{v} L_{v}}{2 D_{h}^{2}}\right)\right]\left(\frac{d x}{d \tau}\right) \\
& +\frac{A \rho_{v} R T}{L_{v}} x \\
= & A \frac{h_{f g} \rho_{v, c}}{T_{e}} \frac{\Delta T_{\max }-\Delta T_{\min }}{2} \\
& +\left(A \frac{h_{f g} \rho_{v, c}}{T_{e}} \frac{\Delta T_{\text {max }}-\Delta T_{\min }}{2}-A \omega u_{\max }\left(L_{l} \rho_{l}+L_{v} \rho_{v}\right)\right) \cos (\omega \tau)
\end{aligned}
$$

Dividing through with $\left(L_{l} \rho_{l}+L_{v} \rho_{v}\right) A$

$$
\begin{aligned}
\frac{d^{2} x}{d \tau^{2}}+ & \frac{A\left[f_{l} \operatorname{Re}_{l}\left(\frac{\mu_{l} L_{l}}{2 D_{h}^{2}}\right)+f_{v} \operatorname{Re}_{v}\left(\frac{\mu_{v} L_{v}}{2 D_{h}^{2}}\right)\right]}{\left(L_{l} \rho_{l}+L_{v} \rho_{v}\right) A}\left(\frac{d x}{d \tau}\right) \\
+ & \frac{\left(\frac{A \rho_{v} R T}{L_{v}}\right)}{\left(L_{l} \rho_{l}+L_{v} \rho_{v}\right) A} x \\
= & \frac{\left(A \frac{h_{f g} \rho_{v, c}}{T_{e}} \frac{\Delta T_{\max }-\Delta T_{\min }}{2}\right)}{\left(L_{l} \rho_{l}+L_{v} \rho_{v}\right) A} \\
+\quad & \frac{\left(A \frac{h_{f g} \rho_{v, c}}{T_{e}} \frac{\Delta T_{\max }-\Delta T_{\min }}{2}-A \omega u_{\max }\left(L_{l} \rho_{l}+L_{v} \rho_{v}\right)\right)}{\left(L_{l} \rho_{l}+L_{v} \rho_{v}\right) A} \cos (\omega \tau)
\end{aligned}
$$

Eq. (34) is similar to the governing equation for forced damped mechanical vibrations, shown below: 


$$
\frac{d^{2} x}{d \tau^{2}}+\frac{c}{m}\left(\frac{d x}{d \tau}\right)+\frac{k}{m} x=\frac{B}{m}+\frac{D}{m} \cos (\omega \tau)
$$

where:

$$
\begin{aligned}
& m=\left(L_{l} \rho_{l}+L_{v} \rho_{v}\right) A \\
& c=A\left[f_{l} \operatorname{Re}_{l}\left(\frac{\mu_{l} L_{l}}{2 D_{h}^{2}}\right)+f_{v} \operatorname{Re}_{v}\left(\frac{\mu_{v} L_{v}}{2 D_{h}^{2}}\right)\right] \\
& k=\frac{A \rho_{v} R T}{L_{v}} \\
& B=A \frac{h_{f g} \rho_{v, c}}{T_{e}} \frac{\Delta T_{\text {max }}-\Delta T_{\min }}{2} \\
& D=A \frac{h_{f g} \rho_{v, c}}{T_{e}} \frac{\Delta T_{\text {max }}-\Delta T_{\min }}{2}-A \omega u_{\text {max }}\left(L_{l} \rho_{l}+L_{v} \rho_{v}\right)
\end{aligned}
$$

\subsection{Two-Phase Heat Transfer with Oscillating Flow}

The two-phase operation of the RMDHL, utilizes reciprocating flow boiling to enhance the heat transfer (many other systems/configurations that employ the same enhance mechanism are still possible) and boiling makes the analytical issues associated with 'oscillating flow cooling' more complicated than those synonymous single-phase reciprocating flow. Some commonality in heat transfer mechanisms would exist. It is also expected that specific underlying heat transfer mechanisms related to the present study would exist, which could significantly different from in the 
single-phase reciprocating flow. One of the most important heat transfer mechanisms for both cases is believed to be the boundary layer development/destruction cycle in conjunction with the fluid mixing under a reciprocating condition.

\subsubsection{Heat Transfer Mechanisms in Two-Phase Operation of the RMDHL}

As shown in Fig. 5.2, when applying a heat source to the external circumference of the evaporator section, heat is transported by radial conduction through the evaporator wall and reaches the working fluid, resulting in vaporization. The heat transfer process for between the working fluid and wall in the RMDHL should be a function of the oscillating frequency, oscillating amplitude, and evaporation/condensation heat transfer associated with oscillating motion. This study

used the methods of Rohsenow (1952), Chen (1966) (J. C. Chen 1966), and Webb and Gupte (1992)(Webb and Gupte 1992) to select a correlation for two phase heat transfer in the RMDHL.

Rohsenow (1952) proposed that the flow boiling could be evaluated by adding the contribution of the pool boiling to that of the single-phase forced convection. Chen (1966) was the first to have formulated a cohesive flow boiling model, and introduced the following relation for the flow boiling heat transfer coefficient:

$$
\begin{gathered}
h=S h_{n b p}+F h_{l} \\
h_{n b p}=0.00122\left[\frac{k_{l}^{0.79} c_{p l}^{0.45} \rho_{l}^{0.49}}{\sigma^{0.5} \mu_{l}^{0.29} h_{l v}^{0.24} \rho_{v}^{0.24}}\right]\left[T_{w}-T_{\text {sat }}\left(P_{l}\right)\right]^{0.24}\left[P_{s a t}\left(T_{w}\right)-P_{l}\right]^{0.75}
\end{gathered}
$$


where $S$ is a suppression factor to account for the reduction in the nuclear boiling heat transfer coefficient due to the suppression of nucleation sites by the forced convection, $h_{n b p}$ is the nuclear boiling heat transfer coefficient and can be evaluated by using an appropriate pool boiling heat transfer coefficient, $F$ is a two-phase convective enhancement factor, and $h_{l}$ is the heat transfer coefficient for single-phase flow alone and can be evaluated by Eq. (40). Webb and Gupte (1992) has proposed the following relations for $S$ and $F$ :

$$
\begin{aligned}
& S=\left(1+2.56 \times 10^{-6} \mathrm{Re}_{t p}^{1.17}\right)^{-1} \\
& F=1+1.8 X_{t t}^{-0.79}
\end{aligned}
$$

where $R e_{t p}$ is Reynolds number for a two-phase flow, and $X_{t t}$ is the Martinelli parameter are defined by:

$$
\begin{gathered}
\operatorname{Re}_{t p}=\operatorname{Re}_{l}\left[F\left(X_{t t}\right)\right]^{1.25} \\
\operatorname{Re}_{l}=\frac{G(1-x) D_{h}}{\mu_{l}} \\
X_{t t}=\left(\frac{1-x}{x}\right)^{0.9}\left(\frac{\rho_{v}}{\rho_{l}}\right)^{0.9}\left(\frac{\mu_{l}}{\mu_{v}}\right)^{0.1} \\
F\left(X_{t t}\right)=1 \quad \text { for } \quad X_{t t}^{0.1} \leq 0.1 \\
\text { else } \quad F\left(X_{t t}\right)=2.35\left(0.213+\frac{1}{X_{t t}}\right)^{0.736}
\end{gathered}
$$

For a known liquid filling ratio, $\Phi$, 


$$
\begin{aligned}
& x=\frac{\rho_{v}-\Phi \rho_{v}}{\bar{\rho}} \\
& \frac{1}{\bar{\rho}}=\frac{x}{\rho_{v}}+\frac{1-x}{\rho_{l}}
\end{aligned}
$$

where $h_{1}$ is the liquid-phase heat transfer coefficient which can be found from section 3.4 as:

$$
\overline{N u}=3.27 W_{o}^{0.2}+\frac{0.0024\left(A / L_{e}\right) W_{o}^{2} \operatorname{Pr}}{1+0.017\left[\left(A / L_{e}\right) W_{o}^{2} \operatorname{Pr}\right]^{2 / 3}}
$$




\section{CHAPTER SIX: CONCLUSIONS}

This study has demonstrated that the RMDHL may be able to handle a heat flux level on the order of $1,000 \mathrm{~W} / \mathrm{cm}^{2}$ and significantly advance the thermal management at various levels. The RMDHL can find significant and broad applications and could become an enabling technology for many critical thermal/fluid related undertakings, including thermal management applications for high-power electronics, electric vehicles, and off-grid energy storage involving Li-ion batteries.

To encourage the adoption of the RMDHL for practical design, this research has proposed two tools that address the scientific issues related to the complicated boiling heat transfer in reciprocating flow:

1. A plot of $k_{\text {eff }}$ against stroke for a given configuration of the RMDHL.

2. A plot of $h$ against stroke for a given configuration of the RMDHL.

3. A plot of $P$ against $V$ for a given configuration of the RMDHL for the estimation of work and efficiency.

4. A correlation for $h$ for a given two-phase configurations of the RMDHL.

5. Mass transport for the slug flow in RMDHL. 
The five parameters above are perhaps the most critical parameters required for the practical design of any RMDHL heat transfer device. Additional operational parameters of the RMDHL include piston stroke of the reciprocating driver, piston reciprocating frequency, and size of the heat loop including the inner diameter of the liquid reservoir and the diameter of the loop tubing. These essential parameters have been systematically varied in this study for broad applications.

A sufficient number of experimental data was collected through this study. These preliminary data were used to determine the best values for the constants in the formulated correlations through a multivariable regression analysis. The correlations thus produced add useful content to the heat transfer archive and can also provide a tool for the design of heat transfer system involving reciprocating flow with configurations different from those of the RMDHL.

A numerical model for the simulation of the reciprocating flow in the RMDHL has been successfully built and validated by the existing experimental data. This numerical modeling has further enhanced the studies of the complex fluid flow and heat transfer mechanisms related to oscillating fluid flow. It will also aid in the design and performance optimization of future RMDHLs. One of the significant procedures implemented in this model is the use of the virtual loop to close the oscillatory loop. Comparison of measured data with the calculated results showed a high degree of agreement between the sets of values. The virtual loop offers a significant advantage for numerical modeling of oscillatory flow as it requires significantly fewer computation resources. 
The results of this study indicate that the cooling efficiency of the RMDHL is meaningfully higher than that of the DPDHL with a reduced maximum temperature under the same heat input and cooling conditions. Additionally, the temperature profiles are much more uniform for an RMDHL loop as compared to those of a DPDHL regarding temperature distribution and cooling efficiency. Considering the other advantages of coolant leak free and the absence of cavitation problems, the RMDHL could be an alternative of a conventional LCS for electronic cooling applications as well as for aerospace-related applications

In a traditional two-phase flow, different flow regimes, such as bubbly, slug, annular, or mist regime would exist. The flow regimes under the present condition were investigated using a numerical model. The numerical and analytical studies have revealed that heat transfer coefficient in the RMDHL is about $30 \%$ to $50 \%$ higher than the coefficients of unidirectional flow. The study also showed that two-phase operations of the RMDHL significantly improve the heat transfer compared to the single-phase reciprocating flow.

A disadvantage of the single-phase unidirectional flow is that a significant wall temperature fluctuation would occur due to a low heat capacity of the single-phase flow. However, the experimental data of single-phase reciprocating flow of this study indicate that at a moderate driver reciprocating frequency of $0.5-1.0 \mathrm{~Hz}$, the temperature at the heated surface was remarkably stable without noticeable fluctuation. However, the combination of the result of the higher heat transfer capacity of the twophase flow and the oscillating velocity profile shows that the behavior of two-phase 
cooling does not follow the expectation that phase change will reduce temperature fluctuations. Although there is a temperature stabilization regime, a second regime exists where the wall temperature fluctuations are relatively significant.

To improve the accuracy of CFD model for the RMDHL, extensive numerical simulations with different turbulence models have been carried out to study fully developed turbulent, low velocity and high heat transfer reciprocating flow. The flow statistics obtained from the numerical results of seven selected turbulent prediction models have been compared with available measurement data with good agreement. The results indicate that for a close prediction of the average cold plate temperature, the standard k- $\varepsilon$ Model models provides the best accuracy while the RNG k- $\varepsilon$ Model offers more accurate information with respect to variations in temperature across the cold plate surface and will generally provide the best qualitative and quantitative basis for additional modeling consideration for future RMDHL study.

This study has proposed a novel method for approximating the power consumption for an oscillating flow using the RMDHL as a case study. The study has also presented an up- to- date compilation of various working equations to quantify multiple parameters associated with the oscillating flow and the details of the various research that provided the correlation. It is the intention that this compilation will be beneficial to the work of applying oscillating flow devices like the RMDHL for many heat transfer applications. 


\section{REFERENCES}

Abramowitz, Milton, and Irene A. Stegun. 1972. Handbook of Mathematical Functions. Edited by Milton Abramowitz and Irene A. Stegun. Dover. http://people.math.sfu.ca/ cbm/aands/intro.htm\#006.

Agostini, Bruno, Matteo Fabbri, Jung E. Park, Leszek Wojtan, John R. Thome, and Bruno Michel. 2007. "State of the Art of High Heat Flux Cooling Technologies." Heat Transfer Engineering 28 (4): 258-81. doi:10.1080/01457630601117799.

Ahn, Kyung H., and Mounir B Ibrahim. 1991. "A 2-D Oscillating Flow Analysis in Stirling Engine Heat Exchangers.” In 1991 Joint ASME-JSME Fluids Engineering Conference, 159-64. Portland, OR.

Akdag, Unal, Mustafa Ozdemir, and A. Feridun Ozguc. 2008. "Heat Removal from Oscillating Flow in a Vertical Annular Channel." Heat and Mass Transfer/Waerme- Und Stoffuebertragung 44 (4): 393-400. doi:10.1007/s00231007-0248-5.

Akdag, Unal, and A. Feridun Ozguc. 2009. "Experimental Investigation of Heat Transfer in Oscillating Annular Flow." International Journal of Heat and Mass Transfer $52 \quad(11-12) . \quad$ Elsevier $\quad$ Ltd: 2667-72. doi:10.1016/j.ijheatmasstransfer.2009.01.006.

Akhavan, R., R. D. Kamm, and a. H. Shapiro. 1991. "An Investigation of Transition to Turbulence in Bounded Oscillatory Stokes Flows Part 2. Numerical Simulations.” Journal of Fluid Mechanics 225 (1): 423. doi:10.1017/S0022112091002112.

Akselband, Boris, Kathryn Whitenack, and Dick Goldman. 2006. “Copper Cold Plate Technology Comparison." Thermomechanical Phenomena in Electronic Systems -Proceedings of the Intersociety Conference 2006: 147-50. 
doi:10.1109/ITHERM.2006.1645335.

Allan, J. 2014. Stirling Cycle Engines Inner Workings and Design. West Sussex: John Wiley \& Sons, Ltd. doi:10.1002/9781118818428.

Aminfar, Habib, Mousa Mohammadporfard, and Rasool Maroofiazar. 2013. "Eulerian Simulation of Subcooled Boiling Flow in Straight and Curved Annuli." Journal of Mechanical Science and Technology 27 (5): 1295-1304. doi:10.1007/s12206013-0501-4.

Andersen, Stig Kildegård, Henrik Carlsen, and Per Grove Thomsen. 2006. "Numerical Study on Optimal Stirling Engine Regenerator Matrix Designs Taking into Account the Effects of Matrix Temperature Oscillations." Energy Conversion and $\begin{array}{lllll}\text { Management } & 47 & \text { (7-8). } & \text { Pergamon: }\end{array}$ doi:10.1016/j.enconman.2005.06.006.

Antal, S.P., R.T. Lahey, and J.E. Flaherty. 1991. "Analysis of Phase Distribution in Fully Developed Laminar Bubbly Two-Phase Flow." International Journal of Multiphase Flow 17 (5): 635-52. doi:10.1016/0301-9322(91)90029-3.

Automotive Energy Supply Corporation. 2016. "Cell, Module, and Pack for EV Applications | Automotive Energy Supply Corporation.” http://www.eco-aesclb.com/en/product/liion_ev/\#anc01.

Barreno, I., S.C. Costa, M. Cordon, M. Tutar, I. Urrutibeascoa, X. Gomez, and G. Castillo. 2015. "Numerical Correlation for the Pressure Drop in Stirling Engine Heat Exchangers." International Journal of Thermal Sciences 97. Elsevier Masson SAS: 68-81. doi:10.1016/j.ijthermalsci.2015.06.014.

Bergles, AE. 2003. "High-Flux Processes through Enhanced Heat Transfer." http://18.7.29.232/handle/1721.1/5564.

Bergman, Theodore L., Adrienne S. Lavine, and Frank P. Incropera. 2012. Fundamentals of Heat and Mass Transfer,. 7thed. Hoboken, NJ: John Wiley. http://www.wiley.com/WileyCDA/WileyTitle/productCd-EHEP001810.html.

Blythman, R, T Persoons, N Jeffers, and DB B Murray. 2016. "Effect of Oscillation Frequency on Wall Shear Stress and Pressure Drop in a Rectangular Channel for 
Heat Transfer Applications." Journal of Physics: Conference Series 745 (3): 32044. doi:10.1088/1742-6596/745/3/032044.

Cao, Yiding, and Mingcong Gao. 2003. "Reciprocating-Mechanism Driven Heat Loops and Their Applications." In ASME 2003 Heat Transfer Summer Conference Heat Transfer: Volume 1, 781-89. Las Vegas, Nevada: ASME. doi:10.1115/HT2003-47195.

—. 2008. "Experimental and Analytical Studies of Reciprocating-Mechanism Driven Heat Loops (RMDHLs)." Journal of Heat Transfer 130 (7): 72901. doi:10.1115/1.2909078.

Cao, Yiding, Dehao Xu, and Mingcong Gao. 2013. "Experimental Study of a BellowsType Reciprocating-Mechanism Driven Heat Loop." International Journal of Energy Research 37 (6): 665-72. doi:10.1002/er.2889.

Chen, Yanyan, Ercang Luo, and Wei Dai. 2007. "Heat Transfer Characteristics of Oscillating Flow Regenerator Filled with Circular Tubes or Parallel Plates." Cryogenics 47 (1). Elsevier Ltd: 40-48. doi:10.1016/j.cryogenics.2006.09.005.

Cheng, Peter D L. 1995. "Experiments in Heat Transfer Under Conditions of Oscillating Pressure and Flow."

Clift, R, Jr Grace, and Me Weber. 2005. Bubbles, Drops, and Particles. Oval Road, London: Academic Press.

Cole, Robert. 1960. "A Photographic Study of Pool Boiling in the Region of the Critical Heat Flux." AIChE Journal 6 (4). American Institute of Chemical Engineers: $533-$ 38. doi:10.1002/aic.690060405.

Cooper, W.L., V.W. Nee, and K.T. Yang. 1994. "An Experimental Investigation of Convective Heat Transfer from the Heated Floor of a Rectangular Duct to a Low Frequency, Large Tidal Displacement Oscillatory Flow.” International Journal of Heat and Mass Transfer 37 (4). Pergamon: 581-92. doi:10.1016/00179310(94)90130-9.

Currie, I G. 2003. "Fundamental Mechanics of Fluids." Mechanical Engineering 1. doi:10.1017/CBO9781107415324.004. 
David C. Wilcox. 2006. Turbulence-Modeling-for-CFD. 3rded. La Canada, CA: DCW industries.

De-Jongh, J., and R Rijs, eds. 2004. Pump Design. Pumps. Veldhoven: Arrakis.

Drew, D A. 1983. "Mathematical Modeling of Two-Phase Flow." Annual Review of Fluid Mechanics 15 (1): 261-91. doi:10.1146/annurev.fl.15.010183.001401.

Faghri, Amir, and Yuwen Zhang. 2006. Transport Phenomena in Multiphase Systems. Burlington, MA: Elsevier Academic Press.

Faghri, Amir, Yuwen Zhang, and John R. Howell. 2010. Advanced Heat and Mass Transfer. Colombia: Global Digital Press. https://books.google.com/books?id=yxMnotbAAz4C\&pgis=1.

Fox, Robert W, Alan T McDonald, and Philip J Pritchard. 2009. Introduction to Fluid Mechanics. 7thed. Vol. 5. John Wiley \& Sons New York.

Frank, Thomas, Jun Mei Shi, and Alan D. Burns. 2004. "Validation of Eulerian Multiphase Flow Models for Nuclear Safety Application." 3rd International Symposium on Two-Phase Flow Modelling and Experimentation Pisa, 22-24 September 2004, no. September: 22-24.

Ghafarian, Mohsen, Davod Mohebbi-Kalhori, and Jafar Sadegi. 2013. "Analysis of Heat Transfer in Oscillating Flow through a Channel Filled with Metal Foam Using Computational Fluid Dynamics." International Journal of Thermal Sciences 66 (April): 42-50. doi:10.1016/j.ijthermalsci.2012.11.008.

Grassmyer, William Alan. 1994. "Experiments in Heat Transfer Under Conditions of Oscillating Pressure and Flow."

Grossman, G, and I Nachman. 2006. "An Analytical Heat Transfer Model for Reciprocating Laminar Flow in a Channel." In Advances in Cryogenic Engineering, 51:1580-88.

Gu, Junping, Qinggong Wang, Yuxin Wu, Junfu Lyu, Suhui Li, and Wei Yao. 2017. "Modeling of Subcooled Boiling by Extending the RPI Wall Boiling Model to Ultra-High Pressure Conditions." Applied Thermal Engineering 124 (September). Pergamon: 571-84. doi:10.1016/j.applthermaleng.2017.06.017. 
Gündoğdu, M.Y., and M.Ö. Çarpınlığlu. 1999. "Present State of Art on Pulsatile Flow Theory, Part 2. Turbulent Flow Regime.” JSME International Journal Series B: Fluids and Thermal Engineering 42 (3): 398-410.

Gundogdu, Mehmet, and Melda Carpinlioglu. 1999. "Present State of Art on Pulsatile Flow Theory: Part 1: Laminar and Transitional Flow Regimes." JSME International Journal Series B Fluids and Thermal Engineering 42 (3): 384-97.

Harris, J, G Peev, and W L Wilkinson. 1969. "Velocity Profiles in Laminar Oscillatory Flow in Tubes." Journal of Physics E: Scientific Instruments 2 (11). IOP Publishing: 913.

Henkes, R.A.W.M., F.F. Van Der Vlugt, and C.J. Hoogendoorn. 1991. "NaturalConvection Flow in a Square Cavity Calculated with Low-Reynolds-Number Turbulence Models." International Journal of Heat and Mass Transfer 34 (2): 377-88. doi:10.1016/0017-9310(91)90258-G.

Hermann, Weston Arthur, Scott Ira Kohn, Kurt Russell Kelty, Clay Hajime Kishiyama, Anil Paryani, Alexander Thomas Jacobs, Grant Dufresne Cutler, and Peng Zhou. 2012. Heat dissipation for large battery packs. US8153290 B2. US Patent ..., issued October 28, 2012. https://www.google.com/patents/US8153290.

Hetsroni, Gad, Albert Mosyak, and Zelik Segal. 2001. "Nonuniform Temperature Distribution in Electronic Devices Cooled by Flow in Parallel Microchannels." IEEE Transactions on Components and Packaging Technologies 24 (1): 16-23. doi:10.1109/6144.910797.

Hu, J.Y., W. Dai, E.C. Luo, X.T. Wang, and Y. Huang. 2010. "Development of High Efficiency Stirling-Type Pulse Tube Cryocoolers." Cryogenics 50 (9): 603-7. doi:10.1016/j.cryogenics.2010.02.015.

Ibrahim, M. B., Zhiguo Zhang, and S Kembhavi. 2002. "A 2-D Axisymmetric CFD Model of Oscillatory Flow with Separation." In Energy Conversion Engineering Conference, 2002. IECEC '02. 2002 37th Intersociety, 37:511-17. IEEE. http://ieeexplore.ieee.org/xpls/abs_all.jsp?arnumber=1392103.

Ibrahim, M B, Zhiguo Zhang, and Rong Wei. 2002. “A 2-D CFD Model of Oscillatory 
Flow with Jets Impinging on a Random Wire Regenerator Matrix." Intersociety Energy Conversion Engineering Conference, 1-7. doi:10.1109/IECEC.2002.1392096.

Ibrahim, Mounir, and Waqar Hashim. 1994. "Oscillating Flow in Channels with a Sudden Change in Cross Section." Computers \& Fluids 23 (1): 211-24. doi:10.1016/0045-7930(94)90035-3.

Iguchi, M., Ohmi, M., \& Maegawa, M. 1982. "Analysis of Free-Oscillating Flow in a U-Shaped Tube." Bulletin of the JSME 25 (207): 1398-1405. doi:10.1248/cpb.37.3229.

Iliadis, G, and P Anagnostopoulos. 1998. "Viscous Oscillatory Flow around a Circular Cylinder at Low Keulegan-Carpenter Numbers and Frequency Parameters." International Journal for Numerical Methods in Fluids 26 (4): 403-42. doi:10.1002/(SICI)1097-0363(19980228)26:4<403::AID-FLD640>3.3.CO;2-M.

Ishii, Mamoru, and Novak Zuber. 1979. "Drag Coefficient and Relative Velocity in Bubbly, Droplet or Particulate Flows." AIChE Journal 25 (5): 843-855. doi:10.1002/aic.690250513.

Jeakins, WD, and WJM Moizer. 2003. "Cooling of Electronic Equipment." US Patent $6,538,881,1-70$.

Jensen, B L, B M Sumer, and J Fredsoe. 1989. “Turbulent Oscillatory Boundary Layers at High Reynolds Numbers." Journal of Fluid Mechanics 206 (1): 265-97. doi:10.1017/S0022112089002302.

Ju, Y.L., C. Wang, and Y. Zhou. 1998. "Numerical Simulation and Experimental Verification of the Oscillating Flow in Pulse Tube Refrigerator." Cryogenics 38 (2): 169-76. doi:10.1016/S0011-2275(97)00139-2.

Justesen, Peter. 1991. "A Numerical Study of Oscillating Flow around a Circular Cylinder." Journal of Fluid Mechanics 222 (1): 157. doi:10.1017/S0022112091001040.

Kang, Sukhvinder, David Miller, and John Cennamo. 2007. "Ipack2007-33870 Closed Loop Liquid Cooling for High Performance Computer." Power, 1-7. 
Kanzaka, Mitsuo, and Makio Iwabuchi. 1992. "Study on Heat Transfer of Heat Exchangers in the Stirling Engine - Performance of Heat Exchangers in the Test Stirling Engine." JSME International Journal 35 (4): 647-52. http://adsabs.harvard.edu/abs/1992JSMEJ..35..647K.

Karagöz, İrfan, and I Karagoz. 2002. "Similarity Solution of the Oscillatory Pressure Driven Fully Developed Flow in a Channel." Uludag University, Muh Min Dergisi 7 (1986): 161-69.

Kim, Do Yun, Chang Hyun Shin, Jun Ha Hwang, Young Real Kim, and Chung Yuen Won. 2016. "The Configuration of EV System Using Battery Module Balancing Method." 2015 18th International Conference on Electrical Machines and Systems, ICEMS 2015, 2122-26. doi:10.1109/ICEMS.2015.7385391.

Kim, S.-E., D Choudhury, and B Patel. 1999. "Computations of Complex Turbulent Flows Using the Commercial Code Fluent." In Modeling Complex Turbulent Flows SE - 15, edited by ManuelD. Salas, JerryN. Hefner, and Leonidas Sakell, 7:259-76. ICASE/LaRC Interdisciplinary Series in Science and Engineering. Springer Netherlands. doi:10.1007/978-94-011-4724-8_15.

Kocamustafaogullari, G., and M. Ishii. 1983. "Interfacial Area and Nucleation Site Density in Boiling Systems." International Journal of Heat and Mass Transfer 26 (9): 1377-87. doi:10.1016/S0017-9310(83)80069-6.

Kolev, Nikolay Ivanov. 2007. Multiphase Flow Dynamics 1: Fundamentals. 2nd ed. New York: Springer-Verlag Berlin Heidelberg. doi:10.1007/978-3-642-20601-6.

_. 2011. Multiphase Flow Dynamics 2: Mechanical Interactions. Vol. 3. Berlin Heidelberg: Springer-Verlag. doi:10.1007/b138146.

Krishnamoorthy, Shriram. 2008. "Modeling and Analysis of Temperature Distribution in Nanoscale Circuits and Packages." University of Illinois at Chicago.

Kuosa, M., K. Saari, A. Kankkunen, and T.-M. Tveit. 2012. "Oscillating Flow in a Stirling Engine Heat Exchanger." Applied Thermal Engineering 45: 15-23. doi:10.1016/j.applthermaleng.2012.03.023.

Kurul, N., and M. Z. Podowski. 1991. "On the Modeling of Multidimensional Effects 
in Boiling Channels." In ANS Proceeding of the 27th National Heat Transfer Conference, 301-14. Minneapolis, Minnesota.

Kurul, N., and M.Z. Podowski. 1990. "Multidimensional Effects in Forced Convection Subcooled Boiling." In Proceedings of the Ninth International Heat Transfer Conference, 19-24. Jerusalem, Israel.

Landau, L D, and E M Lifshitz. 1987. Course of Theoretical Physics - Mechanics. 2nd ed. Nauka: Pergamon Press.

Langtry, R B, F R Menter, S R Likki, Y B Suzen, P G Huang, and S. Völker. 2006. “A Correlation-Based Transition Model Using Local Variables-Part II: Test Cases and Industrial Applications." Journal of Turbomachinery 128 (3): 423-34. doi:10.1115/1.2184353.

Langtry, Robin, and Florian Menter. 2005. "Transition Modeling for General CFD Applications in Aeronautics." In 43rd AIAA Aerospace Sciences Meeting and Exhibit. Aerospace Sciences Meetings. American Institute of Aeronautics and Astronautics. doi:doi:10.2514/6.2005-522.

Launder, B.E., and D.B. Spalding. 1974. "The Numerical Computation of Turbulent Flows." Computer Methods in Applied Mechanics and Engineering 3 (2): 26989. doi:10.1016/0045-7825(74)90029-2.

Lee, Dae-Young, Sang-Jin Park, and Sung Tack Ro. 1998. "Heat Transfer in the Thermally Developing Region of a Laminar Oscillating Pipe Flow." Cryogenics 38 (6): 585-94. doi:10.1016/S0011-2275(98)00020-4.

Leong, K.C., and L.W. Jin. 2005. "An Experimental Study of Heat Transfer in Oscillating Flow through a Channel Filled with an Aluminum Foam." International Journal of Heat and Mass Transfer 48 (2): 243-53. doi:10.1016/j.ijheatmasstransfer.2004.08.025.

Li, Huiying, Sergio A. Vasquez, Hemant Punekar, and R. Muralikrishnan. 2011. "Prediction of Boiling and Critical Heat Flux Using an Eulerian Multiphase Boiling Model." In Volume 6: Fluids and Thermal Systems; Advances for Process Industries, Parts A and B, 463-76. ASME. doi:10.1115/IMECE2011-65539. 
Li, P., and K.T. Yang. 2000. "Mechanisms for the Heat Transfer Enhancement in ZeroMean Oscillatory Flows in Short Channels." International Journal of Heat and Mass Transfer 43 (19): 3551-66. doi:10.1016/S0017-9310(99)00390-7.

Liu, Huaqiang, Zhongbao Wei, Weidong He, and Jiyun Zhao. 2017. "Thermal Issues about Li-Ion Batteries and Recent Progress in Battery Thermal Management Systems: A Review." Energy Conversion and Management 150 (October): 30430. doi:10.1016/j.enconman.2017.08.016.

Liu, Zao, Sheldon Tan, Hai Wang, Yingbo Hua, and Ashish Gupta. 2014. "Compact Thermal Modeling for Packaged Microprocessor Design with Practical Power Maps." Integration, the VLSI Journal 47 (1). Elsevier: 71-85. doi:10.1016/j.vlsi.2013.07.003.

Lopez de Bertodano, Martin A. 1992. "Turbulent Bubbly Two-Phase Flow in a Triangular Duct.” Ann Arbor, Mich.: UMI.

Ma, H. B., M. A. Hanlon, and C. L. Chen. 2006. "An Investigation of Oscillating Motions in a Miniature Pulsating Heat Pipe.” Microfluidics and Nanofluidics 2 (2): 171-79. doi:10.1007/s10404-005-0061-8.

Ma, H. B., C. Wilson, B. Borgmeyer, K. Park, Q. Yu, S. U.S. Choi, and Murli Tirumala. 2006. "Effect of Nanofluid on the Heat Transport Capability in an Oscillating Heat Pipe.” Applied Physics Letters 88 (14): 1-7. doi:10.1063/1.2192971.

Mackley, M. R., and P. Stonestreet. 1995. "Heat Transfer and Associated Energy Dissipation for Oscillatory Flow in Baffled Tubes." Chemical Engineering Science 50 (14): 2211-24. doi:10.1016/0009-2509(95)00088-M.

Mahamud, Rajib, and Chanwoo Park. 2011. "Reciprocating Air Flow for Li-Ion Battery Thermal Management to Improve Temperature Uniformity." Journal of Power Sources 196 (13): 5685-96. doi:10.1016/j.jpowsour.2011.02.076.

Markatos, N.C. 1986. "The Mathematical Modelling of Turbulent Flows." Applied Mathematical Modelling 10 (3): 190-220. doi:10.1016/0307-904X(86)90045-4.

Mashayek, Farzad. 2001. "Dynamics of Evaporating Drops. Part II: Free Oscillations." International Journal of Heat and Mass Transfer 44 (8): 1527-41. 
doi:10.1016/S0017-9310(00)00200-3.

Menon, A.S., M.E. Weber, and H.K. Chang. 1984. "Model Study of Flow Dynamics in Human Central Airways. Part III: Oscillatory Velocity Profiles." Respiration Physiology. doi:10.1016/0034-5687(84)90026-4.

Menter, F. R., R. B. Langtry, S. R. Likki, Y. B. Suzen, P. G. Huang, and S. Völker. 2006. "A Correlation-Based Transition Model Using Local Variables-Part I: Model Formulation." Journal of Turbomachinery 128 (3): 413-22. doi:10.1115/1.2184352.

Menter, F R. 1994. "Two-Equation Eddy-Viscosity Turbulence Models for Engineering Applications." AIAA Journal 32 (8). American Institute of Aeronautics and Astronautics: 1598-1605. doi:10.2514/3.12149.

Menter, Florian R. 2011. "Turbulence Modeling for Engineering Flow.” Canonsburg. http://cfd.spbstu.ru/agarbaruk/c/document_library/DLFE-41517.pdf.

Miyazaki, Y. 2000. Textbook: Oscillating Heat Pipes. doi:10.4271/2000-01-2375.

Monte, F De, G Galli, Localiti Monteluco, Roio Poggio, and L Aquila. 1996. "Heat Exchangers and Regenerator in Stirling Machines," 1421-27.

Moran, Michael J., and Howard N Shapiro. 2004. Fundamentals Of Engineering Thermodynamics. Chemistry \& .... doi:10.1177/004057368303900411.

Moriasi, D.N., J.G. Arnold, M.W. Van Liew, R.L. Binger, R.D. Harmel, and T.L. Veith. 2007. "Model Evaluation Guidelines for Systematic Quantification of Accuracy in Watershed Simulations." Transactions of the ASABE 50 (3): 885900. doi:10.13031/2013.23153.

Munekazu Ohmi, Manabu Iguchi, Ikuo Urahata. 1982. "Flow Patterns and Frictional Losses in an Oscillating Pipe Flow." Bulletin of the JSME 25 (202): 536-43. doi:http://doi.org/10.1299/jsme1958.25.536.

Munson, BR, DF Young, and TH Okiishi. 2009. Fundamentals of Fluid Mechanics. 6thed. Danvers: Wiley.

Nallasamy, M. 1987. "Turbulence Models and Their Applications to the Prediction of Internal Flows: A Review." Computers \& Fluids 15 (2): 151-94. 
Ni, Mingjiang, Bingwei Shi, Gang Xiao, Zhongyang Luo, and Kefa Cen. 2015. "Heat

Transfer Characteristics of the Oscillating Flows of Different Working Gases in U-Shaped Tubes of a Stirling Engine." Applied Thermal Engineering 89: 569-77. doi:10.1016/j.applthermaleng.2015.06.063.

Ohmi, Munekazu, and Manabu Iguci. 1982. "Critical Reynolds Number in an Oscillating Pipe Flow.” Bulletin of JSME 25 (200): 165-72. doi:10.1299/jsme1958.25.165.

Özdinç Çarpinlioğlu, Melda, and Mehmet Yaşar Gündoğdu. 2001. "A Critical Review on Pulsatile Pipe Flow Studies Directing towards Future Research Topics." Flow Measurement and Instrumentation 12 (3): 163-74. doi:10.1016/S09555986(01)00020-6.

Pamuk, Mehmet. 2016. "A New Heat Transfer Correlation for Oscillating Fluid Flow.” Thermal Science, no. 0: 215-215. doi:10.2298/TSCI160126215P.

Park, Jong Chun, Moo Hyun Kim, and Hideaki Miyata. 1999. "Fully Non-Linear FreeSurface Simulations by a 3D Viscous Numerical Wave Tank." International Journal for Numerical Methods in Fluids 29 (6). John Wiley \& Sons, Ltd.: 685703. doi:10.1002/(SICI)1097-0363(19990330)29:6<685::AIDFLD807>3.0.CO;2-D.

Podowski, Michael Z., Ales Alajbegovic, and Necdet Kurul. 1997. "Mechanistic Modeling of CHF in Forced-Convection Subcooled Boiling." Schenectady, New York.

Popoola, Olubunmi, Ayobami Bamgbade, and Yiding Cao. 2016. "A Numerical Model of a Reciprocating-Mechanism Driven Heat Loop for Two-Phase High Heat Flux Cooling." Journal of Thermal Science and Engineering Applications 8 (4): 41006. doi:10.1115/1.4034059.

Popoola, Olubunmi, and Yiding Cao. 2016. "The Influence of Turbulence Models on the Accuracy of CFD Analysis of a Reciprocating Mechanism Driven Heat Loop." Case Studies in Thermal Engineering 8 (September): 277-90. doi:10.1016/j.csite.2016.08.009. 
Popoola, Olubunmi, Soheil Soleimanikutanaei, and Yiding Cao. 2016a. "Numerical

Simulation of a Reciprocating-Mechanism Driven Heat Loop (RMDHL)." Heat Transfer Research. doi:10.1615/HeatTransRes.2016015276.

—. 2016b. "Reciprocating Mechanism-Driven Heat Loop (RMDHL) Cooling Technology for Power Electronic Systems.” In Electronics Cooling, edited by S M Sohel Murshed, Ch. 0. Rijeka: InTech. doi:10.5772/62518.

Popoola, Olubunmi Tolulope, and Yiding Cao. 2015. "Application of a Reciprocatory Driven Heat Loop to High Heat Single Phase Liquid Cooling for Temperature Uniformity." In Proceedings of the OAU Faculty of Technology Conference (OAU TekCONF 2015), 28-37. Ile-Ife, Nigeria.

Ramos, Eduardo, Brian D. Storey, Fernando Sierra, Raúl A. Zúniga, and Andriy Avramenko. 2004. "Temperature Distribution in an Oscillatory Flow with a Sinusoidal Wall Temperature.” International Journal of Heat and Mass Transfer 47 (22): 4929-38. doi:10.1016/j.ijheatmasstransfer.2004.04.033.

Rao, Zhonghao, and Shuangfeng Wang. 2011. "A Review of Power Battery Thermal Energy Management.” Renewable and Sustainable Energy Reviews 15 (9): 455471. doi:10.1016/j.rser.2011.07.096.

Richardson, B. A, and E Tyler. 1929. "The Transverse Velocity Gradient near the Mouths of Pipes in Which an Alternating or Continuous Flow of Air Is Established." Proceedings of the Physical Society 42 (1): 1-15. doi:10.1088/0959$5309 / 42 / 1 / 302$.

Schlunder, Ernst U., ed. 1990. Heat Transfer Atlas: Calculation of Heat Transfer. VDI Verlag.

Schwendig, Frank. 1992. Wärme-Und Impulsaustausch in Regenerativen Gaskreisprozessen. VDI-Verlag.

Scott Huck. 2011. "Measuring Processor Power." White Paper Measuring Processor Power: TDP vs. ACP.

Scotti, Alberto, and Ugo Piomelli. 2001. "Numerical Simulation of Pulsating Turbulent Channel Flow." Physics of Fluids 13 (5). 
Semiconductor Industry Association. 1999. International Technology Roadmap for Semiconductors. Austin, TX: International SEMATECH.

Sert, Cuneyt, and Ali Beskok. 2003. "Numerical Simulation of Reciprocating Flow Forced Convection in Two-Dimensional Channels." Journal of Heat Transfer 125: 403-12. doi:10.1115/1.1565092.

Shabani, Bahman, and Manu Biju. 2015. "Theoretical Modelling Methods for Thermal Management of Batteries.” Energies 8 (9): 10153-77. doi:10.3390/en80910153.

Shankleman, Jessica, Tom Biesheuvel, Joe Ryan, and Dave Merrill. 2017. "We're Going to Need More Lithium.” Bloomberg-Bussinessweek. https://www.bloomberg.com/graphics/2017-lithium-batteryfuture/?utm_content=tech\&utm_campaign=socialfloworganic\&utm_source=twitter\&utm_medium=social\&cmpid\%3D=socialflowtwitter-tech.

Shih, T.H., W.W. Liou, A. Shabbir, Z. Yang, and J Zhu. 1995. “A New K-Epsilon Eddy Viscosity Model for High Reynolds Number Turbulent Flows: Model Development and Validation." Computer $\backslash \&$ Fluids 24 (August): 227-38. doi:10.1016/0045-7930(94)00032-T.

Shin, Hung-tae, and Shigefumi Nishio. 1998. "Oscillation-Controlled Heat-Transport Tube (Heat Transfer Coefficient in Tubes in Heating and Cooling Regions)." Heat Transfer - Japanese Research 27 (6): 415-30. doi:10.1002/(SICI)15206556(1998)27:6<415::AID-HTJ2>3.0.CO;2-I.

Simon, Terrence W, and Jorge R Seume. 1988. "A Survey of Oscillating Flow in Stirling Engine Heat Exchangers."

Steve Hanley. 2016. "What Makes the Tesla $100 \mathrm{kWh}$ Battery so Different?" https://www.teslarati.com/what-makes-tesla-100-kwh-battery-so-different/.

Su, Yan, Jane H. Davidson, and Francis A. Kulacki. 2012. "Numerical Investigation of Fluid Flow and Heat Transfer of Oscillating Pipe Flows." International Journal of Thermal Sciences 54 (April): 199-208. doi:10.1016/j.ijthermalsci.2011.11.021. Su, Yan, Jane H Davidson, and F A Kulacki. 2011. "Fluid Flow and Heat Transfer 
Structures of Oscillating Pipe Flows." World Academy of Science, Engineering and Technology, International Journal of Mechanical, Aerospace, Industrial, Mechatronic and Manufacturing Engineering 5 (9): 1813-1822.

Tanaka, Makoto, Iwao Yamashita, and Fumitake Chisaka. 1990. "Flow and Heat Transfer Characteristics of the Stirling Engine Regenerator in an Oscillating Flow." JSME International Journal. Ser. 2, Fluids Engineering, Heat Transfer, Power, Combustion, Thermophysical Properties 33 (2): 283-89.

Tentner, Adrian, Elia Merzari, and Prasad Vegendla. 2014. "Computational Fluid Dynamics Modeling of Two-Phase Boiling Flow and Critical Heat Flux." In Volume 4: Radiation Protection and Nuclear Technology Applications; Fuel Cycle, Radioactive Waste Management and Decommissioning; Computational Fluid Dynamics (CFD) and Coupled Codes; Reactor Physics and Transport Theory, V004T10A037. ASME. doi:10.1115/ICONE22-30844.

Tomiyama, A. 1998. "Struggle with Computational Bubble Dynamics." Multiphase $\begin{array}{lllll}\text { Science } \quad \text { and } & \text { Technology } & 10 & \text { (4): }\end{array}$ doi:10.1615/MultScienTechn.v10.i4.40.

Touloukian, Y S ; Liley, P E ; Saxena, S C. 1970. "Volume 3 : Thermal Conductivity Nonmetallic Liquids and Gases." IEEE Transactions on Information Theory, 707. doi:10.1109/18.57216.

Tziranis, AK. 1992. "Temperature, Heat Flux, and Velocity Measurements in Oscillating Flows with Pressure Variations." Massachusetts Institute of Technology. http://dspace.mit.edu/handle/1721.1/12790.

Uchida, Shigeo. 1956. "The Pulsating Viscous Flow Superposed on the Steady Laminar Motion of Incompressible Fluid in a Circular Pipe." JZeitschrift Für Angewandte Mathematik Und Physik ZAMP 7 (5): 403-22. doi:10.1007/BF01606327.

—. 1964. "The Pulsating Viscous Flow Superposed on the Steady Laminar Motion of Incompressible Fluid in a Circular Pipe.” JZeitschrift Für Angewandte Mathematik Und Physik ZAMP 19 (1): 117-20. doi:10.1007/BF01606327.

Ünal, H.C. 1976. "Maximum Bubble Diameter, Maximum Bubble-Growth Time and 
Bubble-Growth Rate during the Subcooled Nucleate Flow Boiling of Water up to 17.7 MN/m2." International Journal of Heat and Mass Transfer 19 (6): 643-49. doi:10.1016/0017-9310(76)90047-8.

Vafai, Kambiz. 2002. High heat flux electronic cooling apparatus, devices and systems incorporating same. US 20020135980A1, issued 2002.

Valle, Victor H. Del, and D.B.R. Kenning. 1985. "Subcooled Flow Boiling at High Heat Flux." International Journal of Heat and Mass Transfer 28 (10): 1907-20. doi:10.1016/0017-9310(85)90213-3.

Versteeg, K., and H Malalasekera. 2007. An Introduction to Computational Fluid Dynamics: The Finite Volume Method. Vol. M. Essex: Pearson.

Walters, D. Keith, and Davor Cokljat. 2008. "A Three-Equation Eddy-Viscosity Model for Reynolds-Averaged Navier-Stokes Simulations of Transitional Flow." Journal of Fluids Engineering 130 (12): 121401-1-14. doi:10.1115/1.2979230.

Walther, C, H. -D. Kühl, and S Schulz. 2000. "Numerical Investigations on the Heat Transfer in Turbulent Oscillating Pipe Flow." Heat and Mass Transfer 36 (2): 135-41. doi:10.1007/s002310050375.

Walther, Ch., H -D. Kühl, Th. Pfeffer, and S Schulz. 1998. "Influence of Developing Flow on the Heat Transfer in Laminar Oscillating Pipe Flow." Forschung Im Ingenieurwesen 64 (3): 55-63. doi:10.1007/PL00010849.

Wang, Lei, and Xi-Yun Lu. 2004. "An Investigation of Turbulent Oscillatory Heat Transfer in Channel Flows by Large Eddy Simulation." International Journal of Heat and Mass Transfer 47 (10-11): 2161-72. doi:10.1016/j.ijheatmasstransfer.2003.11.010.

Wang, Qingsong, Ping Ping, Xuejuan Zhao, Guanquan Chu, Jinhua Sun, and Chunhua Chen. 2012. "Thermal Runaway Caused Fire and Explosion of Lithium Ion Battery." Journal of Power Sources 208 (June 2012). Elsevier B.V.: 210-24. doi:10.1016/j.jpowsour.2012.02.038.

West, C. D. 1982. "Two-Phase, Two-Component Stirling Engine with Controlled Evaporation." United States. 
Woodbank Communications Ltd. 2005. "Energy Conversion and Heat Engines." http://www.mpoweruk.com/heat_engines.htm.

Xiao, Gang, Conghui Chen, Bingwei Shi, Kefa Cen, and Mingjiang Ni. 2014. "Experimental Study on Heat Transfer of Oscillating Flow of a Tubular Stirling Engine Heater." International Journal of Heat and Mass Transfer 71: 1-7. doi:10.1016/j.ijheatmasstransfer.2013.12.010.

Xiao, Gang, Tianxue Zhou, Mingjiang Ni, Conghui Chen, Zhongyang Luo, and Kefa Cen. 2014. "Study on Oscillating Flow of Moderate Kinetic Reynolds Numbers Using Complex Velocity Model and Phase Doppler Anemometer." Applied Energy 130: 830-37. doi:10.1016/j.apenergy.2014.02.005.

Xiaoguo, Tang, and P. Cheng. 1993. "Correlations of the Cycle-Averaged Nusselt Number in a Periodically Reversing Pipe Flow." International Communications in Heat and Mass Transfer 20 (2). Pergamon: 161-72. doi:10.1016/07351933(93)90045-W.

Yakhot, Victor, and Leslie M. Smith. 1992. "The Renormalization Group, the EExpansion and Derivation of Turbulence Models." Journal of Scientific Computing 7 (1): 35-61. doi:10.1007/BF01060210.

Yang, Naixing, Xiongwen Zhang, BinBin Shang, and Guojun Li. 2016. "Unbalanced Discharging and Aging due to Temperature Differences among the Cells in a Lithium-Ion Battery Pack with Parallel Combination.” Journal of Power Sources 306 (February): 733-41. doi:10.1016/j.jpowsour.2015.12.079.

Young, Kwo, Caisheng Wang, Le Yi Wang, and Kai Strunz. 2013. Electric Vehicle Integration into Modern Power Networks. doi:10.1007/978-1-4614-0134-6.

Zeitoun, O, and M Shoukri. 1996. "Bubble Behavior and Mean Diameter in Subcooled Flow Boiling." Journal of Heat Transfer 118 (1). ASME: 110-16.

Zhang, J., C. Dalton, and R.J. Brown. 1993. "A Numerical Comparison of Morison Equation Coefficients for Low Keulegan-Carpenter Number Flows: Both Sinusoidal and Nonsinusoidal." Journal of Fluids and Structures 7 (1): 39-56. doi:10.1006/jfls.1993.1004. 
Zhao, Ming, Liang Cheng, and Hong-wei An. 2010. "Three-Dimensional Numerical Simulation of Flow around a Circular Cylinder under Combined Steady and Oscillatory Flow." Journal of Hydrodynamics, Ser. B 22 (5): 144-49. doi:10.1016/S1001-6058(09)60184-0.

Zhao, T., and P. Cheng. 1995. "A Numerical Solution of Laminar Forced Convection in a Heated Pipe Subjected to a Reciprocating Flow." International Journal of Heat and Mass Transfer 38 (16): 3011-22. doi:10.1016/0017-9310(95)00017-4.

Zhao, T. S., and P. Cheng. 1996a. "Experimental Studies on the Onset of Turbulence and Frictional Losses in an Oscillatory Turbulent Pipe Flow." International Journal of Heat and Fluid Flow 17 (4): 356-62. doi:10.1016/0142727X(95)00108-3.

— 1996b. "Oscillatory Heat Transfer in a Pipe Subjected a Periodically Reversing Flow." ASME Journal of Heat Transfer 118: 592-98.

—. 1998a. "A Numerical Study of Laminar Reciprocating Flow in a Pipe of Finite Length.” Applied Scientific Research 59: 11-25.

Zhao, T S, and P Cheng. 1998b. "Heat Transfer in Oscillatory Flows." In Annual Review of Heat Transfer, edited by C.L. Tien, IX:359-419. Begell House Inc.

Zhao, T S, and P.Cheng. 1996. "The Friction Coefficient of a Fully-Developed Laminar Reciprocating Flow in a Circular Pipe.” Int. J. Heat and Fluid Flow 17 (96): 16772.

Zuo, ZJ, LR Hoover, and AL Phillips. 2002. "An Integrated Thermal Architecture for Thermal Management of High Power Electronics." In Thermal Challenges in Next Generation Electronic Systems, edited by Joshi \& Garimella, 317-36. Millpress, Rotterdam. 
APPENDIX 1

266 


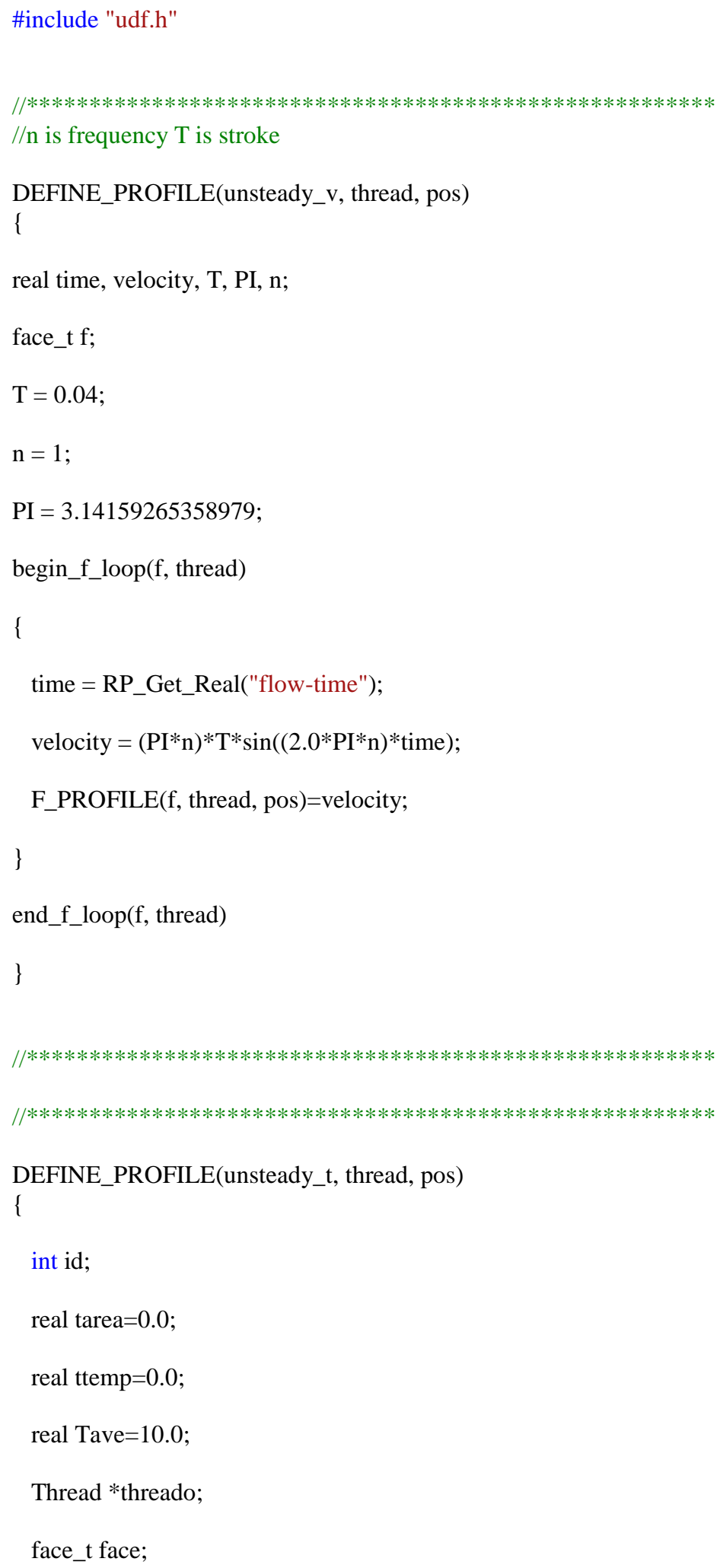




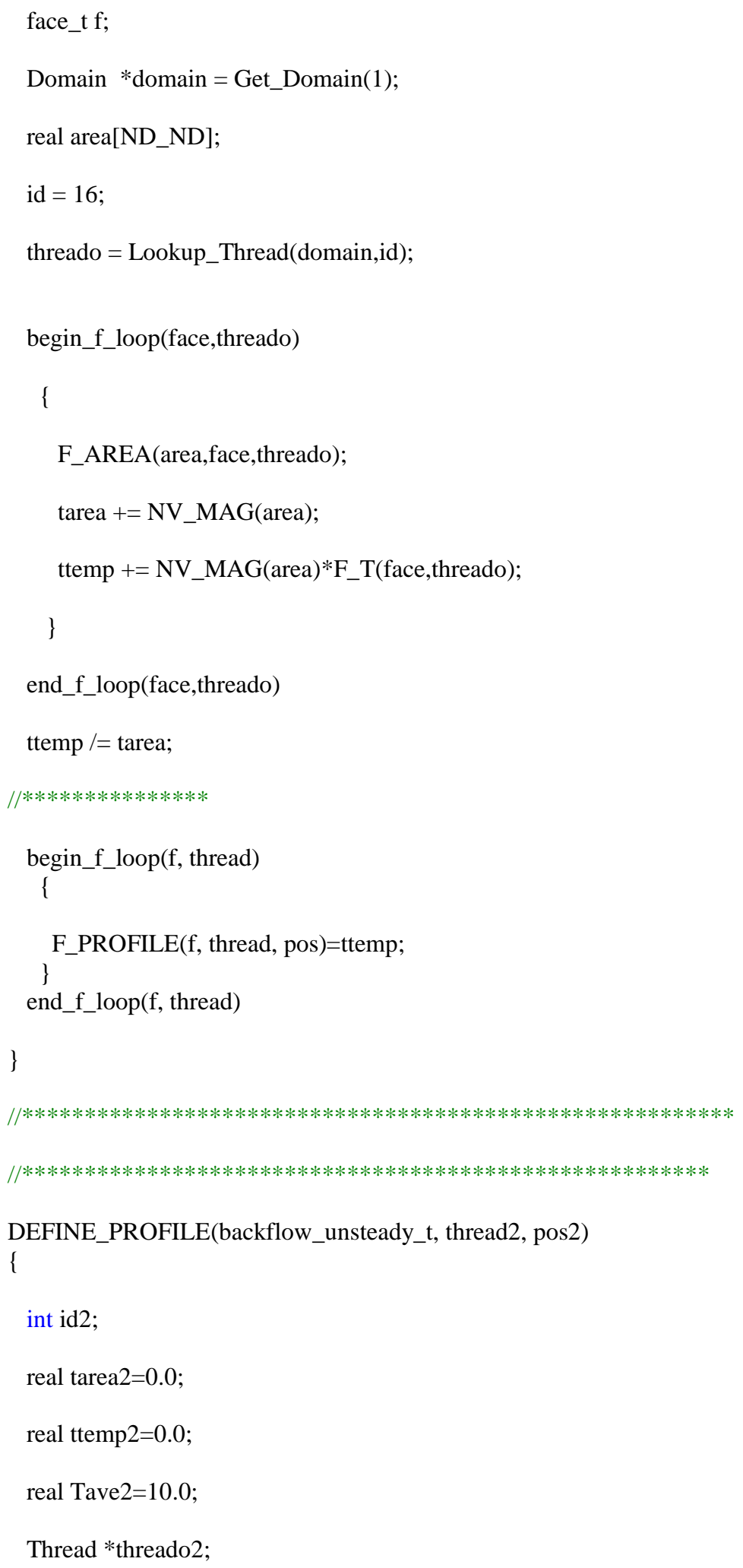




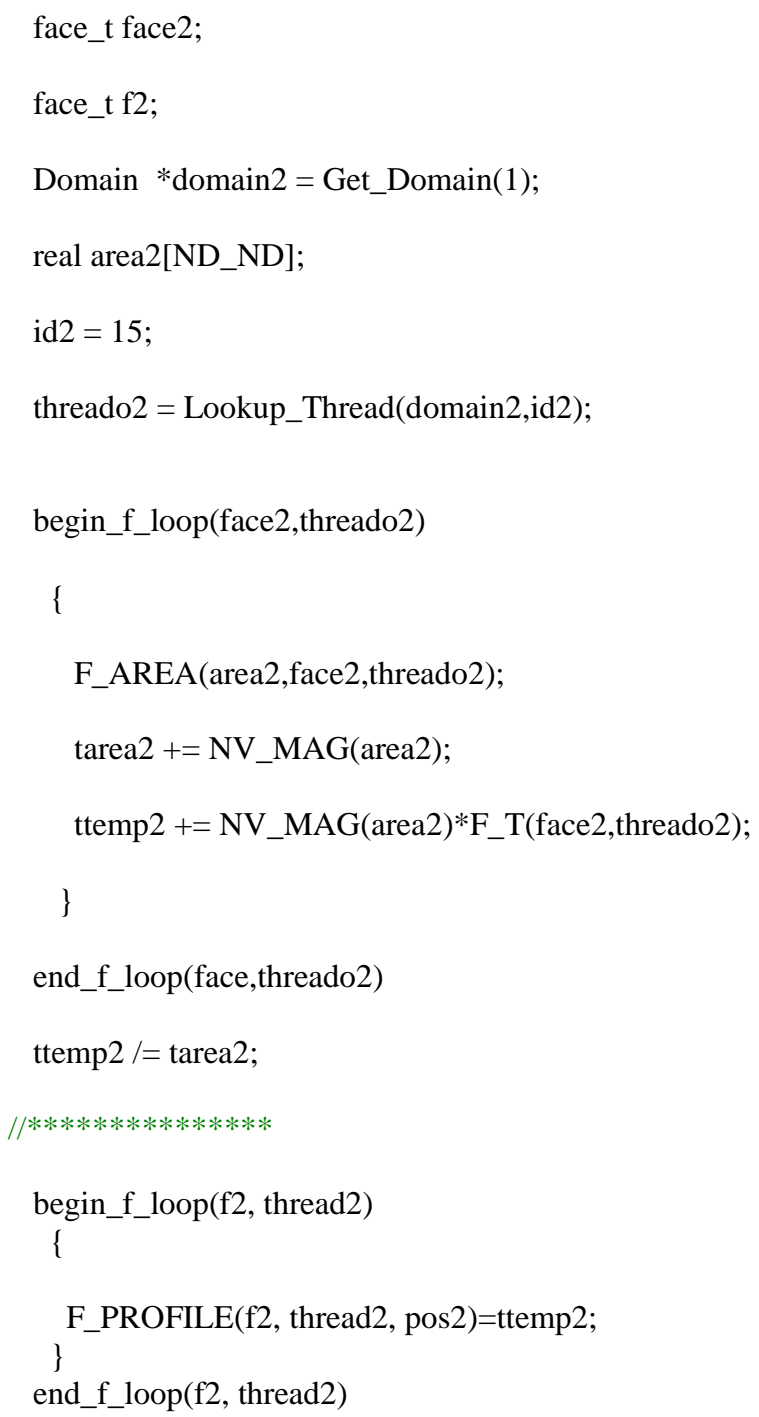


APPENDIX 2 


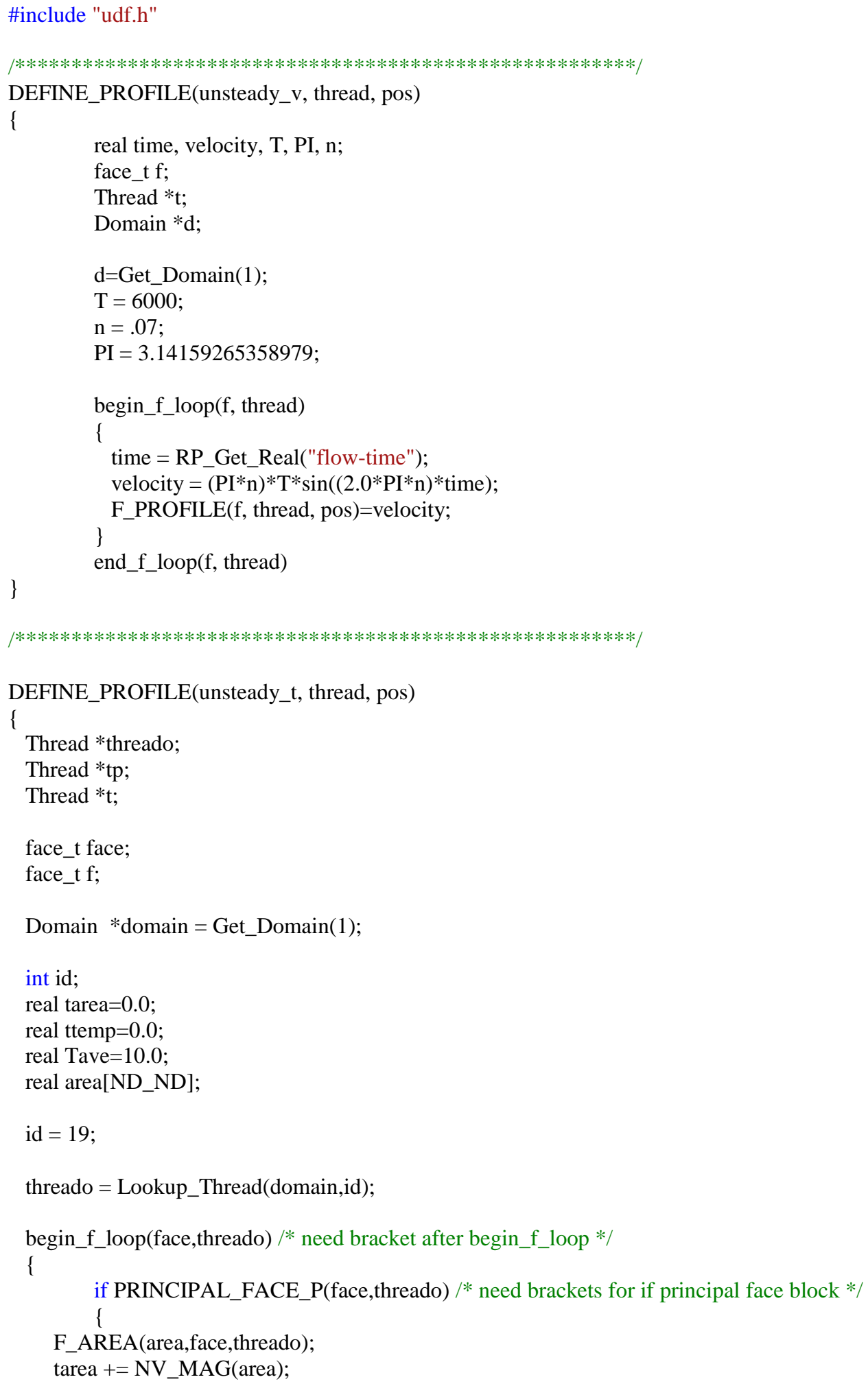




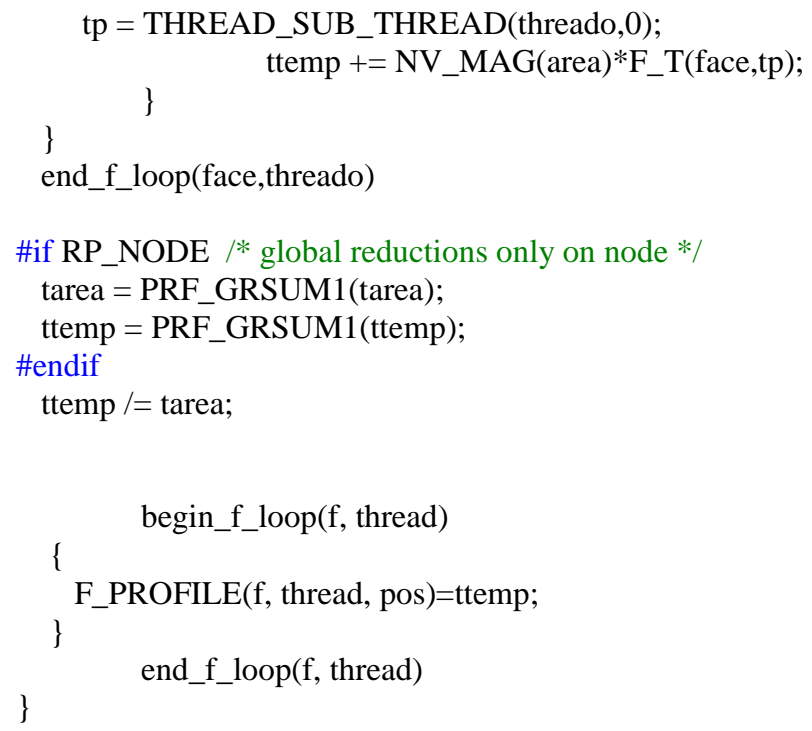




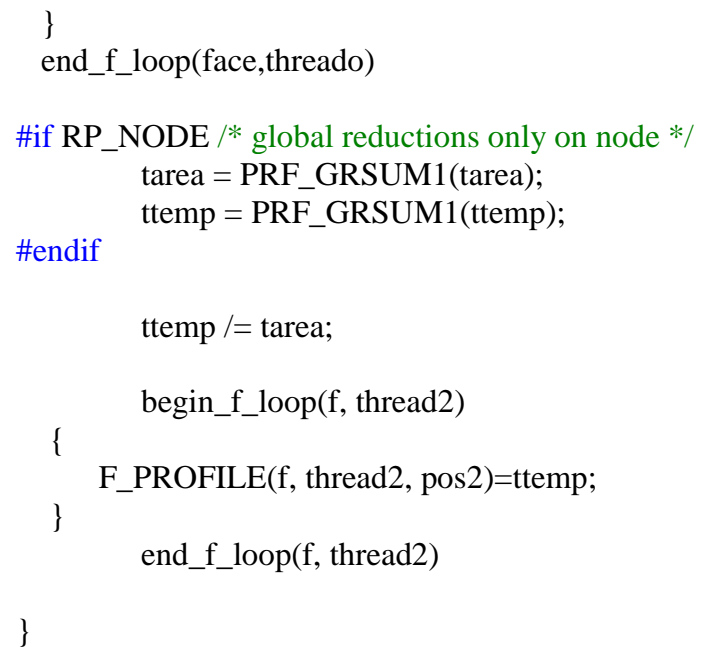


APPENDIX 3 


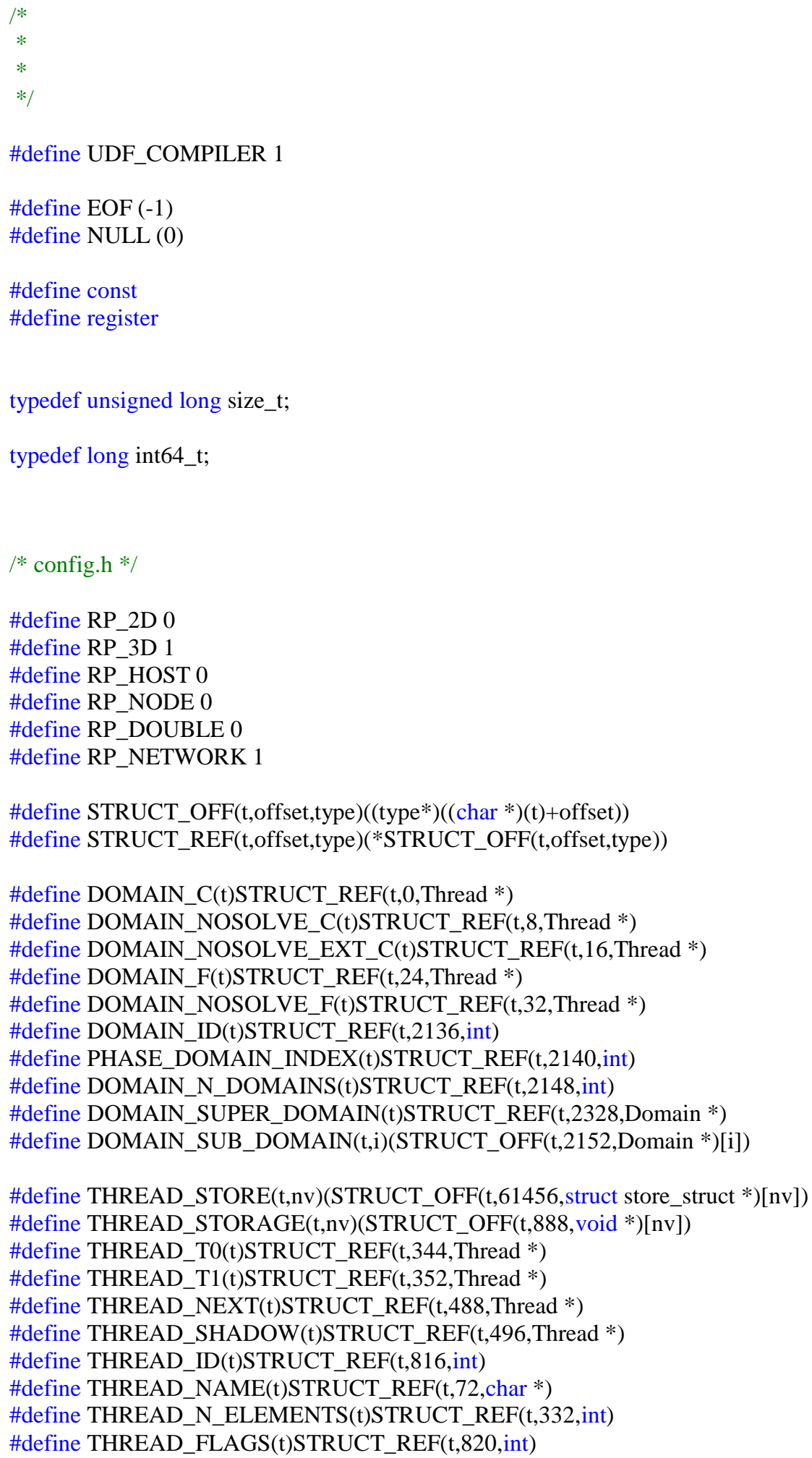




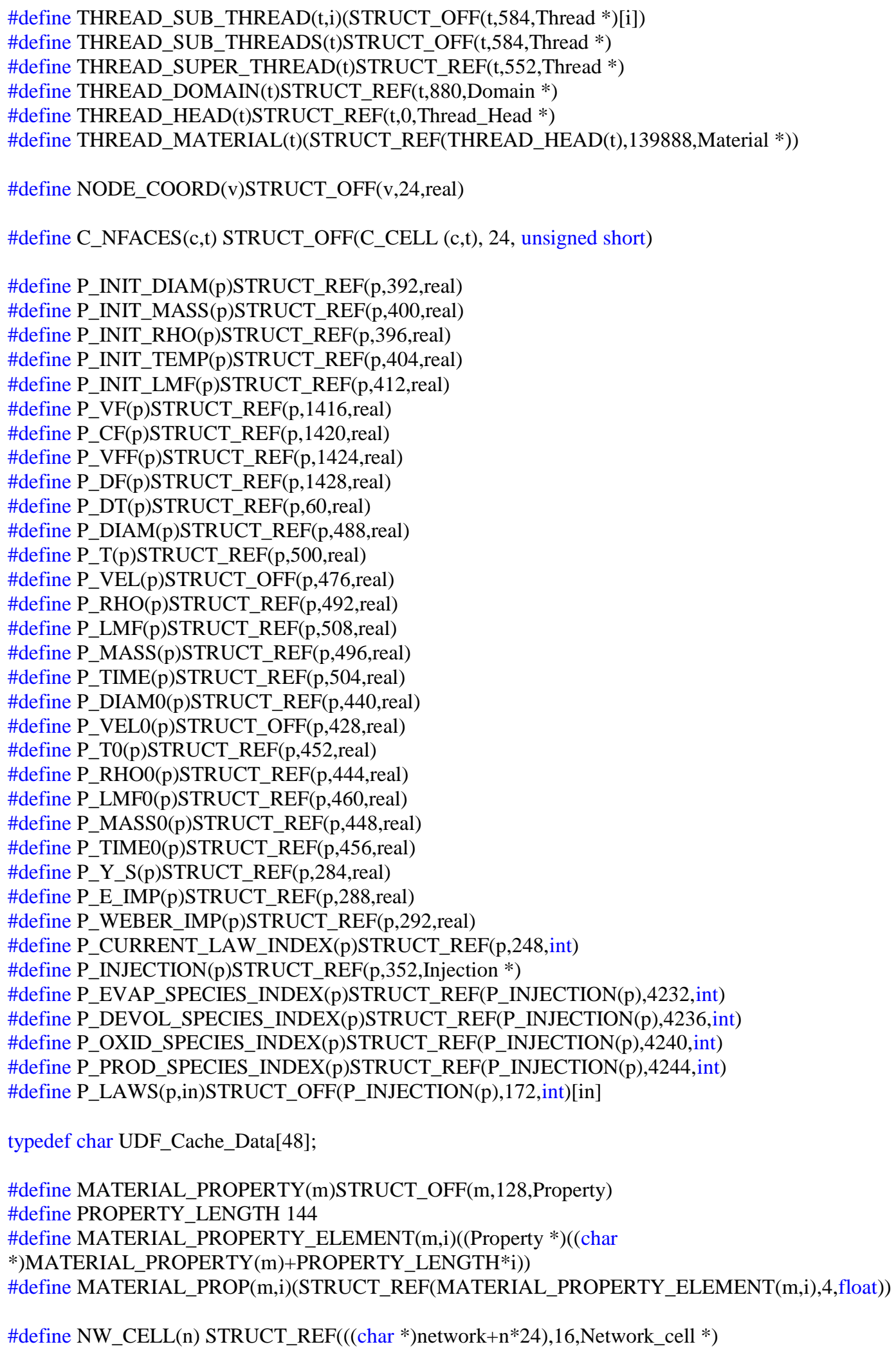


\#define NWC_T(n,c) STRUCT_REF(((char *)NW_CELL(n)+c*360),200,real)

\#define NWE_T(e) STRUCT_REF(((char*)nw_end + e*252),4,real) 
VITA

\section{OLUBUNMI TOLULOPE POPOOLA}

2002-2010

FISTAB Nigeria Ltd, Mushin, Lagos - Project

Manager

2004

Bachelor of Science

Mechanical Engineering

Obafemi Awolowo University

Department of Mechanical Engineering, Obafemi Awolowo University, Ile-Ife, Nigeria Graduate Assistant/Assistant Lecturer/Lecturer

2011

Master of Science

Mechanical Engineering

Obafemi Awolowo University

2013-2017

Florida International University, Miami, USA

Teaching Assistant, Mechanical Engineering

2017

Doctor of Philosophy

Mechanical Engineering

Florida International University

TESLA, Inc. Palo Alto, California;

Battery Safety and Research, Summer Internship

\section{PUBLICATIONS}

1. Popoola, Olubunmi, and Yiding Cao, "The Influence of Turbulence Models On the Accuracy of CFD Analysis of a Reciprocating Mechanism Driven Heat Loop." Case Studies in Thermal Engineering doi:

http://dx.doi.org/10.1016/j.csite.2016.08.009

2. Popoola, O. T., Bamgbade A. \& Cao, Y. (2016) A Numerical Model of a Reciprocating-Mechanism Driven Heat Loop (RMDHL) for Two-Phase High heat Flux Cooling ASME J. Thermal Sci. Eng. Appl; doi: 10.1115/1.4034059

3. Adewole, B Z, Popoola, O. T., and Asere A. A. (2015). Thermal Performance of a Reflector Based Solar Box Cooker Implemented in Ile-Ife, Nigeria. International Journal of Energy Engineering, 5(5): 95-101. 
4. Popoola, O. T., Adio, S. A., Oke, A. O., \& Asere, A. A. (2015). Development of a bench-scale fluidized bed combustor (FBC) for coal and biomass combustion. Nigerian Journal of Technological Research, 10(1), 17-24.

5. Muritala, A. O., Popoola, O. T., Law, C., \& Skews, B. W. (2013). The flow field induced by a blast wave traversing on a convex curved wall. International Journal of Fluid Mechanics Research. 40(5):420-428.

6. Popoola, O. T. \& Asere, A. A. (2013). Emission and Combustion Characteristics of Lafia-Obi Coal in Fluidized Bed Combustor. Advanced Materials Research, 824, 318-326.

7. Muritala, A. O., Popoola, O. T., \& Adio, S. A. (2012). Impart Of Wall Geometry On Thermal Distribution Behind A Diffracted Shock Wave. International Journal of Scientific and Engineering Research, 4(5), 946-954.

8. Malomo, O. B., \& Popoola, O. T. (2010). A design for local production of aluminum alloy (al 6063) based on the optimization of the elastoplastic response of castings. Ife Journal of Technologyof Tech, 19(1), 58-65

9. TFEC-IWHT2017-17562; Popoola, O. T., \& Cao, Y. (2017). Numerical modelling of two-phase reciprocating flow using a virtual loop, $2^{\text {nd }}$ Thermal and Fluids Engineering Conference April 02-05, 2017, The Rio Hotel \& Casino Las Vegas, Nevada, USA

10. Popoola, O. T., Ayobami A Bamgbade, and Y Cao. 2016. "Evaluation of Turbulence Models for the Numerical Study of Reciprocating-Mechanism Driven Heat Loop." In Volume 1B, Symposia: Fluid Mechanics (Fundamental Issues and Perspectives; Industrial and Environmental Applications); Multiphase Flow and Systems (Multiscale Methods; Noninvasive Measurements; Numerical Methods; Heat Transfer; Performance); Transport Phe, Washington, DC: ASME, V01BT25A009. http:// dx.doi.org/10.1115/FEDSM2016-7771.

11. Popoola, O. T., \& Cao, Y. (2015). Application of a Reciprocatory driven heat loop to high heat single phase liquid cooling for temperature uniformity. In Proceedings of the OAU Faculty of Technology Conference (OAU TekCONF 2015)(pp.28-37)). Ile-Ife, Nigeria.

12. Malomo, B. O., Ogunbodede, A. T., Oluwasegun, K. M., Popoola, O. T., Ibitoye, S. A., \& Adekoya, L. O. (2015). A design optimization of the high pressure die casting process for the fabrication of bulk metal matrix composites. In Proceedings of the OAU Faculty of Technology Conference (OAU TekCONF 2015)(pp.294-301). Ile-Ife, Nigeria. 